\title{
Mechanisms in Surface ENHANCED RAMAN SCATTERING
}

by

\section{Matthias Meyer}

\author{
A thesis \\ submitted to the Victoria University of Wellington \\ in fullfilment of the requirements for the degree of \\ Doctor of Philosophy \\ in Physics \\ Victoria University of Wellington \\ The MacDiarmid Institute for Advanced Materials \\ and Nanotechnology \\ 2009
}





\begin{abstract}
This thesis focusses on a number of topics in surface enhanced Raman scattering (SERS). The aim of the undertaken research was to deepen the general understanding of the SERS effect and, thereby, to clarify some of the disputed issues, among them: What is the origin of the enhancement? What is the physical or chemical effect of 'salt activation' in SERS systems? Can we observe single-molecules using SERS? Can we determine the absorbate's orientation on the surface?

In part one (chapters 1-3), as a general introduction, I start with a short overview of the Raman effect and its relation to other molecular spectroscopic effects (such as fluorescence, Rayleigh scattering, etc... ). Following these basic remarks, the surface enhancement mechanisms underlying SERS are explained (as a largely electromagnetic field enhancement) and are investigated theoretically on the canonical model of a nanoscopic dimer of silver spheres.

The second part (chapter 4 ) reports on the experimental investigation (electron microscopy, in-situ Raman measurements) of a typical real SERS system: Lee \& Meisel silver colloids. An emphasis is put on the self-limiting aggregation kinetics which is observed in such systems after salt addition. This is also investigated and rationalised by means of Monte-Carlo simulations which are footed on empiric theoretical considerations for the interaction potential.

Part three (chapter 5) contains a discussion of the early attempts on singlemolecule SERS and points out the shortcomings of the previously used ultra-low concentration approach. In response, an improved and more rigorous approach is presented: Bi-Analyte SERS. Examplary applications of the technique are discussed. Within these experiments the capability of the technique to prove/disprove (with statistical soundness) single-molecule sensitivity in any SERS system is demonstrated, and single-molecule enhancement factors are derived.
\end{abstract}


The last part (chapter 6) presents computational studies based on densityfunctional theory and its use in the context of Raman spectroscopy and SERS. Of particular interest here were the Raman tensors, their visual representation appropriate in the SERS case, their relation to the relative intensities of Raman peaks, and their modification when the photon energy approaches the electronic resonance of the molecule.

Last, but not least, a conclusion chapter is presented, where I highlight what has contributed by the thesis to the general understanding of the SERS effect. 


\section{Acknowledgement}

There was no time for scholarly details, and, besides, I have always believed that a man can fairly be judged by the standards and taste of his choices in matters of high-level plagiarism.

Hunter S. Thompson

It is no secret that a PhD thesis is not the product of a single person. It is much more the collaborative work of many, written up by a single author. Accordingly, this thesis was made possible only through the work and lasting support of many people. A few stand out: most notably, Pablo Etchegoin and Eric Le Ru, who provided a great working environment by being open-minded, patient, hard-working and yet constantly approachable and helpful colleagues. The entire Raman group carries this spirit, and, in addition to Pablo and Eric, I wish to also express particular gratitude to Andrew Preston, Chris Galloway, Evan Blackie, Joe Trodahl and Ben Ruck, who all made, in one way or another, significant contributions to this thesis. The list of reasons documenting why it is a good idea to do your PhD in this group is far too long to exhaust here.

I also wish to thank, for their continuous support, the people of the MacDiarmid Institute and the School of Chemical and Physical Sciences: Margaret Brown, Paul Callaghan, Richard Blaikie, John Spencer, John Lekner, Alan Rennie, and many more (including entire soccer teams!), too numerous to fit into this small frame. 
I am grateful to the financial support of the MacDiarmid Institute and Victoria University of Wellington, without which this work would have been impossible.

Finally, I am forever indebted to my family and friends who I left behind in my hometown Berlin, for their encouragement, understanding and sacrifice. Their unfathomable love and trust remains untouched, despite my arguably rare attempts to bridge the geographical distance via phone, or even email. 


\section{Contents}

1 Introduction 9

2 Molecular spectroscopy 13

2.1 Molecular states and transitions . . . . . . . . . . . . 13

2.2 The Raman effect . . . . . . . . . . . . . . . . 18

2.3 Experimental setup and materials . . . . . . . . . . 28

3 Surface-enhancement mechanisms 35

3.1 Electromagnetic surface enhancement . . . . . . . . . 35

3.1.1 Enhancement factors .............. 36

3.1.2 Dielectric function of noble metals . . . . . . . . . 40

3.1.3 Solving the electromagnetic problem . . . . . . . . 46

3.1.4 Local field of a planar surface . . . . . . . . . . . 48

3.1.5 Local field of a dimer of spheres . . . . . . . . 50

3.2 Chemical enhancement - controversy . . . . . . . . . . . 62

4 Self-limiting colloidal aggregation in SERS-active solutions 66

4.1 Noble metal colloids in SERS . . . . . . . . . . . . 66 
4.1.1 Introduction . . . . . . . . . . . 67

4.1.2 Lee \& Meisel silver colloids . . . . . . . . . . . . . . . 69

4.1.3 Some puzzling observations . . . . . . . . . . . 72

4.2 DLVO theory . . . . . . . . . . . . . . . . . 73

4.2.1 Screened Coulomb repulsion . . . . . . . . . . . 75

4.2.2 van der Waals attraction . . . . . . . . . . 76

4.2.3 Application to Lee \& Meisel colloids . . . . . . . . . . 80

4.3 Electron microscopy of single colloids . . . . . . . . . 86

4.3.1 Investigated samples . . . . . . . . . . . . . . . . . 88

4.3.2 Single colloids and dimers . . . . . . . . . . . . . 88

4.3.3 Aggregates - small and large structures . . . . . . . 89

4.3.4 Rod-like colloidal structures . . . . . . . . . . . . . . 93

4.4 In-situ observation of colloidal aggregation . . . . . . . . 95

4.4.1 The build-up and origin of SERS signals in liquid . . 95

4.5 Monte Carlo simulations . . . . . . . . . . . . . . . . . . . . . 105

4.5.1 Formulating the many-body problem . . . . . . . 105

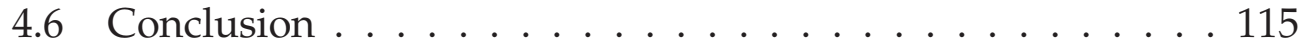

5 Single-molecule SERS and Bi-Analyte SERS 116

5.1 Early SM-SERS claims $\ldots \ldots \ldots 116$

5.1.1 The ultra-low concentration approach . . . . . . . . 117

5.1.2 Poisson distribution - quantised intensities? . . . . . 120

5.2 Recent SM-SERS efforts . . . . . . . . . . . . . . . . . . . . . 124 
5.3 Rigorous proof of SM-SERS using two analytes . . . . . . . 126

5.3.1 The Bi-Analyte SERS idea . . . . . . . . . . . . . . 126

5.3.2 Discrete model hot-spots . . . . . . . . . . . . . 127

5.3.3 Prerequisite criteria . . . . . . . . . . . . . . . 130

5.3 .4 BiASERS - experiment . . . . . . . . . . . . 131

5.3 .5 Caveats . . . . . . . . . . . . . . . . . . 140

5.3.6 BiASERS applications . . . . . . . . . . . . . 143

5.4 Conclusion . . . . . . . . . . . . . . . . . . . . . 150

6 Quantum chemistry 151

6.1 Introduction . . . . . . . . . . . . . . . 152

6.2 Physical background . . . . . . . . . . . . . . . 153

6.3 Computational models . . . . . . . . . . . . . . . . 156

6.4 Applications of DFT _ . . . . . . . . . . . . . . . 157

6.5 Obtaining the Raman tensors . . . . . . . . . . . . 164

6.6 Raman tensor visualisation $\ldots \ldots \ldots \ldots$

6.7 Raman tensors of benzotriazole . . . . . . . . . . . . . 170

6.8 Connection to molecular orientation $\ldots \ldots \ldots$. . . . 176

6.9 Moving towards resonance $\ldots \ldots \ldots \ldots$

6.10 Conclusion . . . . . . . . . . . . . . . . . 186

$\begin{array}{lll}7 & \text { Conclusions } & 187\end{array}$

A Molecular footprints 191 
B BiASERS - from Pascal's triangle to Mie theory

C Artwork

201

D Publications 


\section{Chapter 1}

\section{Introduction}

I don't know anything, but I do know that everything is interesting if you go into it deeply enough.

Richard Feynman

I started the work for this thesis in early 2005, in a newly formed group ${ }^{1}$ which had joined the field roughly one year earlier. After an initial phase of learning the physical foundations of the Raman effect, and after getting accustomed to the experimental realities of surface-enhanced Raman spectrocopy (SERS), the primary research goal was as simple as profound: develop and improve the understanding of the SERS effect. This research undertaken to achieve this goal was mostly, but not solely, experimental in nature. A variety of techniques were employed: optical spectroscopy and microscopy, electron microscopy; also, suitable statistical data analysis software was developed and computational methods were used to predict and understand results. This broad range is reflected in the topics covered throughout the chapters of this thesis, justifying the rather non-specific title "Mechanisms in Surface-Enhanced Raman Spectroscopy".

\footnotetext{
${ }^{1}$ At this point, the initial group consisted solely of Pablo Etchegoin and Eric Le Ru.
} 


\section{Motivation}

SERS is a powerful tool for physics, chemistry, biochemistry and related fields. There are two key reasons for this: (i) SERS inherits its high molecular specificity from the Raman effect [1,2], which allows detailed analytic chemistry with the ability to distinguish miniscule (even isotopic) changes in molecules and (ii) SERS provides large enhancements of the Raman signal, thereby compensating for the small cross section, i.e. low intensity, of Raman scattering. The Raman effect is understood [3], but the signal enhancement provided by a complex mixture of metal nanoparticles with dye and salt raises a whole plethora of questions and problems to investigate.

Clearly, in retrospective, it is always easy to formulate the questions which correspond to the findings and insights gained through one's work. However, a number of general questions emerged early, prior to any serious investigation, and mostly pertaining to the real, experimental system:

- What do the colloids actually look like?

- Which effects constitute the enhancement in SERS?

- Why do SERS samples require activation using salt?

- Is single molecule SERS a reality?

These basic questions provided the initial momentum for this work and ultimately were the driving force leading to many different, sometimes unexpected, results. This thesis addresses these questions and points at some of the developments which branched from these initial objectives.

Another substantial part of this work did not have such an initial motivation; rather, it drew its momentum from my passion for computing, but quickly developed from a hobby into a useful tool: density functional theory (DFT). If one was to assign an initial interest, it would be the question: "Is it advanced enough to yield Raman spectra of sufficient accuracy to be 
useful in the experimental context?" A chapter of this thesis is dedicated to the research based on, or supported by, this computational method.

\section{Thesis outline}

An introductory discussion of molecular spectroscopy and the Raman effect, accompanied by a brief summary of the experimental equipment and materials, is given in chapter 2. Chapter 3 provides an overview of the physics underlying the surface-enhancement effect, with an emphasis on the theoretically predicted enhancements for a model mimicking the real system: metal colloids. The characterisation of the colloidal system is undertaken in chapter 4, which also addresses the effect of salt-based SERSactivation, and, by the same token, shines light onto colloidal aggregation. Chapter 5 is dedicated to the discussion and experimental proof of single molecule SERS. The last part of this thesis, chapter 6, portrays DFT and its use in the SERS context, with a particular emphasis on Raman tensors and the possibility to elucidate molecular orientations in SERS. The thesis is accompanied by a number of appendices.

Before launching into the main body of this work, I will provide a brief outline of the historical timeline of the development of SERS.

\section{Short history of Surface-Enhanced Raman Spectroscopy}

In 1974, experiments by Fleischmann et al. [4], in which pyridine was deposited onto electrochemically roughened silver electrodes, lead to the first observation of surface-enhanced Raman spectra. The enhancement was attributed to the enlarged surface area of the electrode. In 1977, Jeanmaire and van Duyne [5], as well as Albrecht and Creighton [6] conducted similar studies on only slightly roughened silver electrodes and deduced large enhancements incompatible with the interpretation of an increased surface area. Alternatively, Albrecht and Creighton drew a connection to 
the interaction of the probe molecule with surface plasmons of the metal substrate (based on an suggestion by Philpott [7]). Jeanmaire and van Duyne reported a proportionality of the signal to the fourth power of the electromagnetic (em) field, thus also proposing an em effect as cause for the enhancement.

Despite these advances, interest and major progress in SERS came to a halt for nearly two decades. In 1997 the field abruptly re-gathered momentum following the reports by Kneipp et al. [8] and Nie [9], who independently claimed single molecule sensitivity using SERS in colloidal solutions. Claimed enhancement factors reached up to $10^{15}$, thus boosting the effective Raman scattering cross section into the realm of fluorescence, for which single molecule sensitivity was an established reality [10, 11]. However, until recently, single-molecule SERS (SM-SERS) was questioned due to the ambiguity of the statistically weak results $[9,12,13]$. In fact, the settlement of this discussion is one of the major results of this work [14]. 


\section{Chapter 2}

\section{Molecular spectroscopy}

In the beginning the Universe was created.

This has made a lot of people very angry and has been widely regarded as a bad move.

Douglas Adams

This dissertation is almost entirely dedicated to one particular part of the greater science of spectroscopy: the Raman effect. However, a holistic approach to understanding Raman spectroscopy requires -at the very leasta minimal outline of its wider context, and its setting and relation therein.

\subsection{Molecular states and transitions}

In Figure 2.1 a Jablonski diagram ${ }^{1}$ schematically depicts the molecular states together with the light-induced transitions between these levels. The energetic states are commonly categorised as follows:

1. Electronic states (thick horizontal lines) - these are the energy levels of the electronic states of an unperturbed molecule. The energy gap

\footnotetext{
${ }^{1}$ named after Polish physicist Aleksander Jabłoński
} 


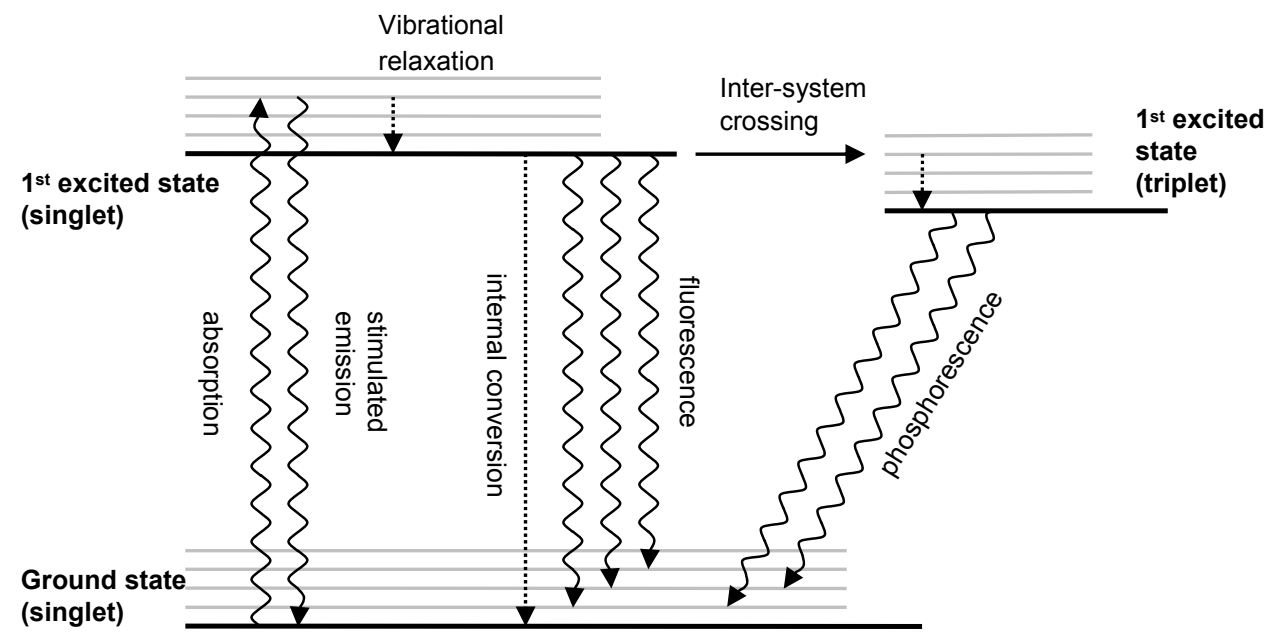

Figure 2.1 - Jablonski diagram showing the electronic (thick horizontal lines) and vibronic (thin horizontal lines) states of a generic molecule (separated vertically to depict difference in energy) and the photon-induced transitions in between these states: radiative transitions (wiggly arrows), non-radiative internal relaxations (dotted arrows) and the spin-forbidden inter-system crossing (short horizontal arrow). See text for more details.

in between electronic states of an organic molecule can be as low as

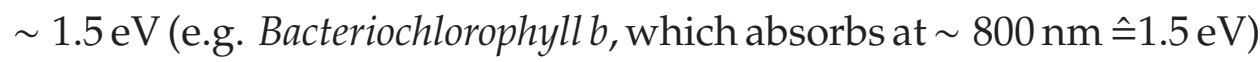
or as large as $\sim 6 \mathrm{eV}$ (e.g. pyrrol, which absorbs at $\sim 210 \mathrm{~nm} \hat{=} 6 \mathrm{eV}$ ). If all electrons are in their lowest possible states, the molecule resides in its ground state, which is, according to Pauli's exclusion principle, in general a singlet $\operatorname{state}^{2}(S=0$, spin multiplicity $2 S+1)$.

2. Vibronic states (thin horizontal lines) - these are sub-states of the electronic states, wherein the electron's molecular orbitals are now perturbed by internal vibrations of the atoms around their equilibrium positions. Hence the name: 'vibrational-electronic' becomes 'vibronic'.

3. Rovibronic states (not drawn) - these are the sub-states to each electronic and vibronic state arising from the quantised molecular rota-

\footnotetext{
${ }^{2}$ Molecular oxygen being a notable exception; it is a spin-triplet in its ground state.
} 
tion.

Between these levels a number of transitions occur:

- Absorption - the vertical excitation of an electron into an energetically higher state (see Franck-Condon principle in Ref. [15]). For a photon to be absorbed, (i) its energy needs to match the gap in between the ground and an excited state, (ii) the transition must be allowed (e.g. not spin forbidden) and (iii) the wave-functions of the ground and excited state need to overlap (Franck-Condon factor). In general, the molecule is excited into a vibronic state; since the number of (ro)vibronic states is large, the absorption spectrum is generally broadened and spans the density of sub-states of the excited electronic state.

- Vibrational relaxation - the fast relaxation into the lowest electronic excited state following absorption. In this case, 'fast' refers to a lifetime measured best in femto- or picoseconds, which is, usually, short in comparison to the lifetime of the excited electronic state (in dyes, typically $\tau \sim O(1 \mathrm{~ns}))$.

- Stimulated emission - photon triggered radiative relaxation.

- Internal conversion - non-radiative relaxation into the ground state; the energy is dissipated through solvent interaction into heat.

- Fluorescence - spin-conserving radiative relaxation into the ground state. This process is subject to the same selection rules as absorption, hence, the vertical de-excitation populates vibronic ground states (leading in general to a broad emission spectrum).

- Intersystem crossing - a spin-flip of the excited electron, leading to a triplet state. If energetically allowed, this otherwise spin-forbidden transition becomes possible through the electron's spin-orbit coupling. The triplet state is of particular importance in molecular 
bleaching: efficient energy transfers to molecular oxygen become possible, leading to oxygen radicals and thus triggering photochemical reactions.

- Phosphorescence - spin-flipping radiative relaxation into the ground state. Much like fluorescence, the phosphorescence spectrum is broad, but of longer lifetime and hence much weaker in intensity, since the transition is spin-forbidden.

\section{Density of states}

Absorption and luminescence ${ }^{3}$ can be observed in atoms, where, since only electronic states are present (i.e. no vibrations), the resulting spectra are discrete (e.g. Balmer series, etc...). A molecule, by the same token, is a quantum object, hence has discrete states - however, absorption and luminescence spectra are generally broad and continuous, leading to the question: What is the density of states of the molecule?

As in the atom's case, the purely electronic energy levels in a molecule are discrete, and usually referred to as $\mathrm{S}_{0}, \mathrm{~S}_{1}\left(\mathrm{~T}_{1}\right)$ etc. for singlet (triplet) states. As mentioned above, there are vibronic sub-states to each of the electronic states. How many vibrations are possible in a molecule? As explained in Fig. 2.2, a molecule with $N$ atoms, after subtracting rigid motion and rotation, has $3 N-6$ degrees of freedom ( $3 N-5$ in linear molecules). This is the number of vibrations, or, more accurately, normal modes possible in the geometric system. For example, benzene with $N=12$ has 30 vibrations, $\mathrm{CO}_{2}$ has four, since it is linear. In each of these vibrations quantised amounts of energy can be stored. Hence, the 'population' of a vibration corresponds to a position in an energy ladder. This contributes heavily to the observed density of states: in the exemplary case of benzene, even if one only accounts for the first three quanta in each vibronic state, this accounts to 90 states trailing towards higher energies from each electronic

\footnotetext{
${ }^{3}$ The term luminescence encompasses both, fluorescence and phosphorescence.
} 


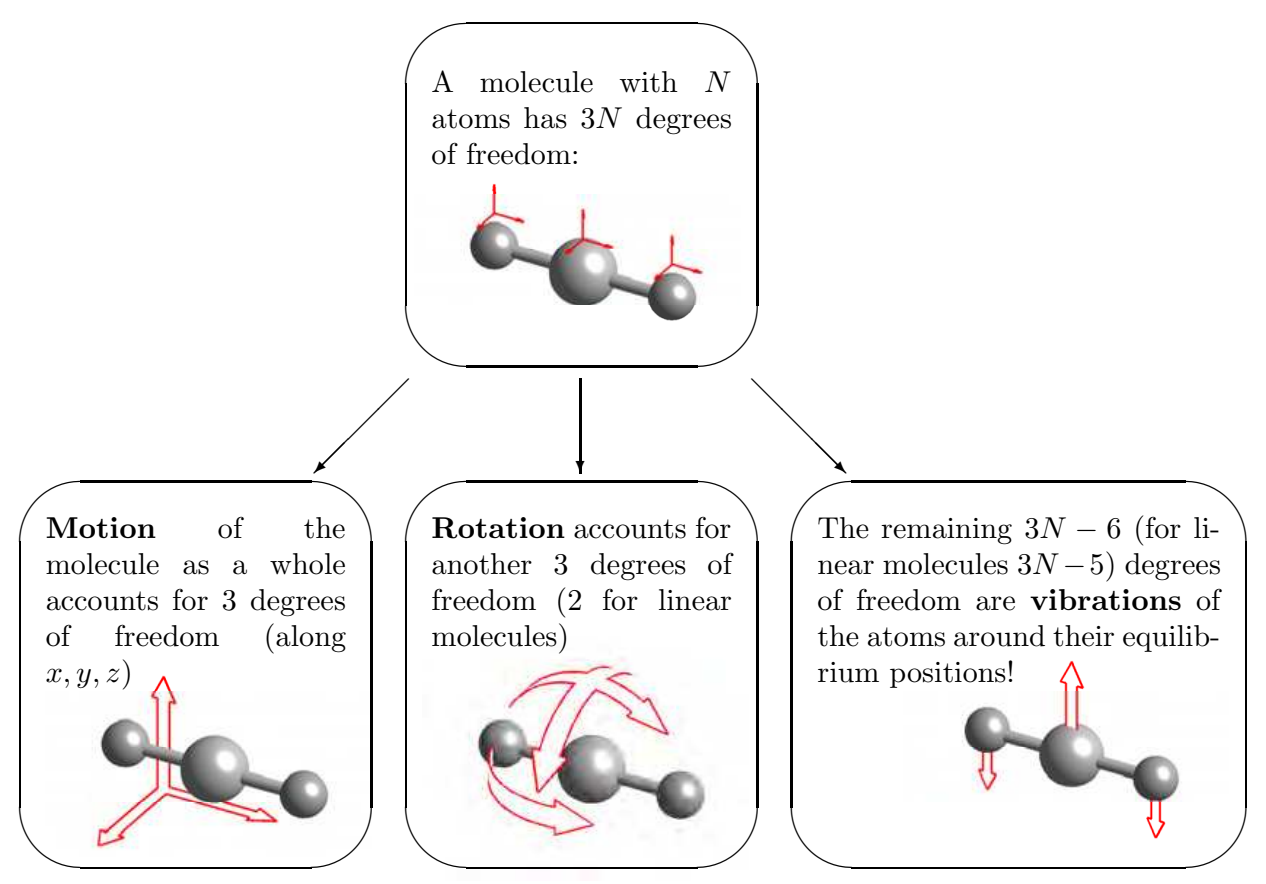

Figure 2.2 - Schematic breakdown of the degrees of freedom in a molecule of $\mathrm{N}$ atoms.

state. The states will be broad in general (lifetime broadening, etc.), thus resulting in a quasi-continuum.

Another quantised sub-set of states adds to the overall density of states: rigid rotation of the molecule. These states are, together with the vibronic states mentioned above, referred to as rovibronic states ${ }^{4}$. Molecular rotation corresponds to quantised states which are possible for any molecular vibronic state, and hence add another 'dimension' to the discrete density of states in a molecule. The hierarchy of states can be summarised as follows:

1. Top-tier are electronic ground state and excited states, in which vibrations are not populated $(n=0)$ and only contribute with the sum of zero-point energies to the overall energy of the molecule.

2. From any purely electronic state, and for each of the possible vibra-

\footnotetext{
${ }^{4} \mathrm{~A}$ concise summary on spectroscopic terms is found in Ref. [15].
} 
tions in a molecule, a set of energetically equidistant states (for small n) extends. These are the vibronic states.

3. Molecular rotation is quantised, thus for all electronic and vibronic states another set of equidistant states extends. These are the rovibronic states.

With the discussion above it becomes apparent that the depiction in Fig. 2.1 of the sub-set of states associated with each electronic state, is an understatement - there is a nested hierarchy of states for each electronic state, caused by vibrations and rotations, which amounts to a high density of states and explains the continuous nature of transitions like fluorescence.

With these preliminary considerations on the energetic structure of molecules, and the photophysical processes possible therein, the spectroscopic foundation to the introduction of the Raman effect is present.

\subsection{The Raman effect}

The Raman effect is the inelastic scattering of photons. The case of interest here is the scattering by phonons, or molecular vibrations. The effect is understandable in classical terms, which is the level of theory this short introduction will focus on. A more formal derivation of the effect requires to treat at least the molecule in quantum mechanical terms; ideally, one would also lift the photon field from its classical description into quantum electrodynamics. For more in-depth treatments see Refs. [3, 16, 17].

Scattering of light on molecules can be elastic, i.e. Rayleigh scattering, or inelastic, i.e. Raman scattering. In the elastic case, the photon's energy, and the state of the molecule after the scattering event are unchanged hence Rayleigh scattered light does not contain much information on the structure of molecular states. In Raman scattering, two inelastic processes can occur: 
- Stokes process: an incident photon $h v_{L}$ excites a molecular vibration $h v_{i}$ and is thus scattered with the corresponding difference in energy $h\left(v_{L}-v_{i}\right)$ (red-shift).

- anti-Stokes process: the photon acquires vibrational energy and is scattered with a higher energy $h\left(v_{L}+v_{i}\right)$ (blue-shift), thus 'cooling' the molecule. This however can take place only if a vibrational quantum $h v_{i}$ is present in the molecule prior to the scattering event, for example due to thermal excitation (governed by the Boltzmann probability $\sim \exp \left(-\frac{h v_{i}}{k_{B} T}\right)$ ) or from a preceeding Stokes process.

\section{Toy model}

For photon scattering on molecules, one can invoke an elementary analogy: a bouncing ball. This didactic toy-model is shown in Figure 2.3; it applies to Rayleigh and Raman scattering:

- In the elastic collision event (representing Rayleigh scattering) the ball bounces off the target back to its original level, i.e. energy is conserved, photons keep their frequency $v_{\mathrm{L}}$.

- In Stokes scattering the ball/photon, upon impact, loses some of its energy to the reduced mass $\mu_{i}$ vibrating with a spring of force constant $k_{i}$. Photons are red-shifted, towards smaller frequencies, by the Raman shift: $v_{i}=\frac{1}{2 \pi} \sqrt{\frac{k_{i}}{\mu_{i}}}$.

- In anti-Stokes scattering, upon impact the ball/photon gains a 'push' from a previously (or thermally) excited vibration. Photons are blueshifted and gain precisely the energy lost in a Stokes event.

\section{Classical description of the Raman effect}

Similarly to other spectroscopic transitions (see above), the Stokes and antiStokes processes can be visualised, more formally, in a Jablonksi diagram, 


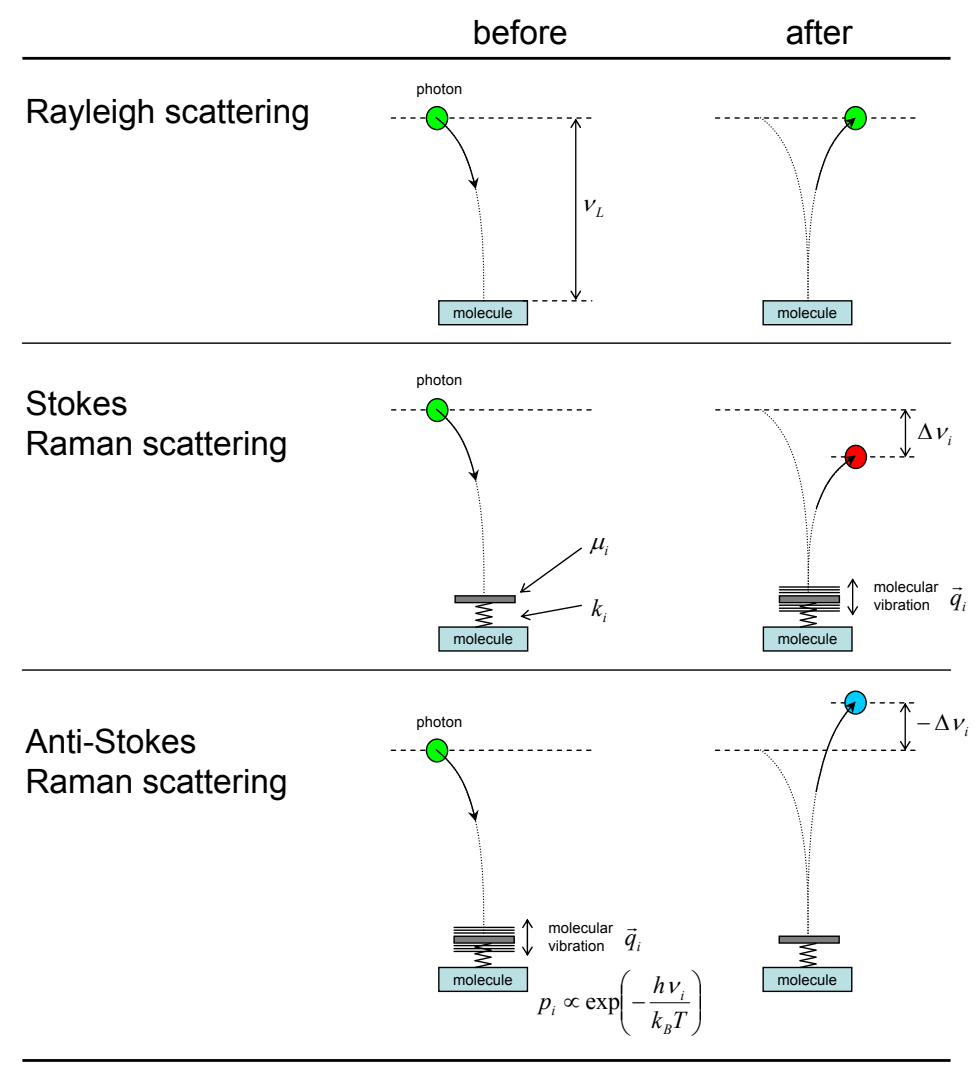

Figure 2.3 - Rayleigh, Stokes and anti-Stokes Raman scattering explained by a simple kinematic analogy - an elastic ball is bounced off an object. The situation is depicted before (1st column) and after (2nd column) the scattering event. For Rayleigh scattering (1st row) the ball (photon with energy $v_{L}$ ) bounces off the object (molecule) elastically, neither losing nor gaining energy from the collision (scattering) event. In Stokes Raman scattering (2nd row) the molecule is able to vibrate internally (harmonic oscillation along a normal mode $\vec{q}_{i}$ ), with a spring constant $k_{i}$ and a reduced mass of the vibration $\mu_{i}$. The impinging photon upon collision induces a vibration, looses the according energy and thus changes its frequency by $\Delta v_{i}$. In anti-Stokes Raman scattering (3rd row) the molecule vibrates prior to the scattering event, usually due to thermal excitation (hence the Boltzmann-probability). The impinging photon now takes energy from the vibration, thus being scattered as a photon that gained energy. 
as shown in Fig. 2.4. The two insets in the top of Fig. 2.4 show the generalisation of the Jablonski diagram to Stokes and anti-Stokes scattering. The inelastic scattering is represented as a two-step process, involving 'absorption' of the incident radiation into a virtual-state and 'emission' from this state into a vibrational or electronic ground state. Despite the explanatory two-step model it is important to keep in mind that the scattering process is in fact instantaneous. The bottom plot shows the resulting spectrum: Rayleigh-scattered laser in the center, Stokes shifted Raman spectrum on the right and anti-Stokes to the left. Note the exponential decay in the mode intensity on the anti-Stokes side.

In physical terms, the origin of the Raman effect is two-fold: (i) the perturbation of the molecule by the incident field and (ii) the modification of the molecule's polarisability through its vibration.

\section{Field perturbation}

The field perturbation alone explains elastic (Rayleigh) scattering. As illustrated in Fig. 2.6A (see top row), an external electromagnetic (em) field $\vec{E}$ (i.e. incident photon) distorts the molecule's electron density, thus leading to a charge displacement and dipole moment $\vec{p}$. The magnitude relating field and dipole is the polarisability $\hat{\alpha}$, which in general is a secondrank tensor ${ }^{5}$ :

$$
\vec{p}_{0}=\hat{\alpha}_{0} \vec{E} \quad,
$$

Assuming the field to oscillate with frequency $\omega_{L}$, i.e.:

$$
\vec{E}=\operatorname{Re}\left[\overrightarrow{E_{0}} \exp (-i \omega t)\right] \quad,
$$

the resulting driven dipole will oscillate, and hence radiate, at the same frequency:

$$
\overrightarrow{p_{0}}=\hat{\alpha}_{0} \overrightarrow{E_{0}} \operatorname{Re}[\exp (-i \omega t)]
$$

\footnotetext{
${ }^{5} \mathrm{~A}$ second rank tensor transforms vectors into vectors; here $\vec{E}$ is transformed into $\vec{p}$.
} 


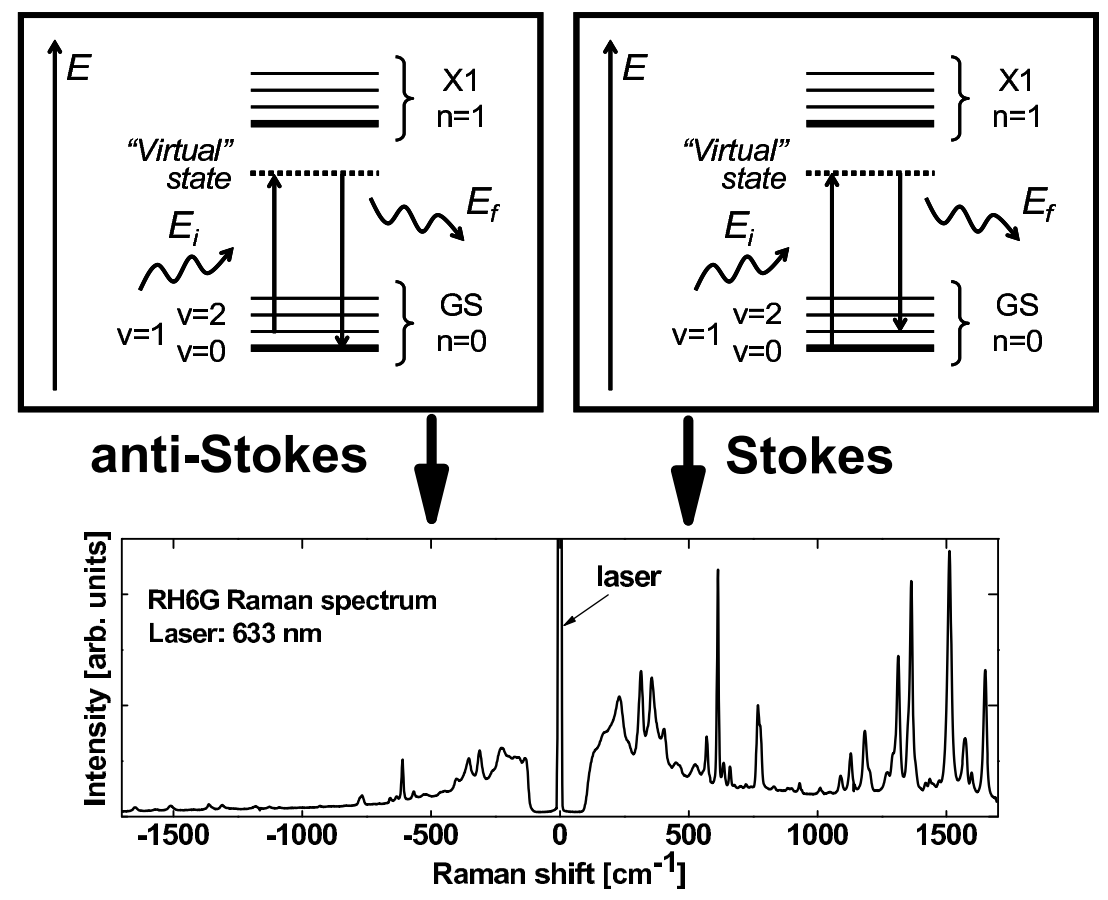

Figure 2.4 - Jablonski diagrams depicting the Stokes Raman process (upper right), the anti-Stokes Raman process (upper left) and Raman spectrum of Rhodamine 6G (lower half, showing both anti-Stokes and Stokes side). In the Stokes Raman process the incident photon creates a molecular vibration and is thus scattered with an accordingly reduced energy (red-shift). In the anti-Stokes process the incident photon picks up energy from an initially existing molecular vibration and is thus scattered with a higher energy (blue-shift). Molecular vibrations exist due to thermal excitation and are in equilibrium with the internal vibrational relaxation of the molecule. Therefore the populations of vibrational states decrease exponentially with their energy (with respect to the ground state). Accordingly the intensity of the anti-Stokes Raman lines decreases with the (negative) Raman shift, e.g. the $1650 \mathrm{~cm}^{-1}$ line is visible on the Stokes, but not on the anti-Stokes side. 


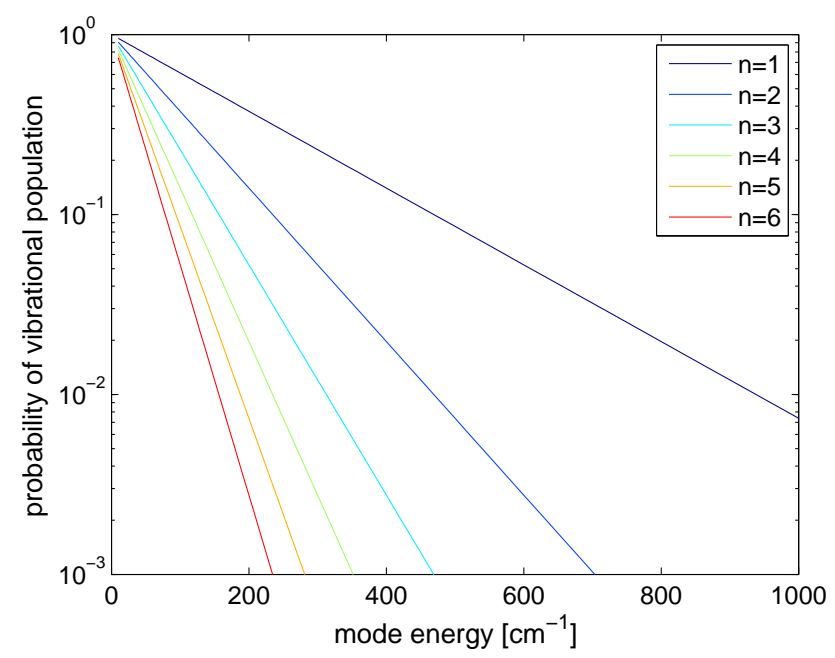

Figure 2.5 - Plot of Boltzmann probability of the population of vibrations at room temperature. The cases for the first three quanta in each vibration are shown. Evidently, a vibration at $1000 \mathrm{~cm}^{-1}$ has a probability of less than $1 \%$ to be excited at all, whereas a mode at $100 \mathrm{~cm}^{-1}$ has a $23 \%$ probability to be triply excited $(n=3)$.

\section{Vibrational perturbation}

The electron density in a molecule is perturbed in the presence of a vibration. Spatially, vibrations are characterised through normal coordinates [18], which provide a complete basis of orthogonal displacement vectors: $\overrightarrow{q_{i}}$ for each mode $i$, with elements $q_{i}^{j}$ for each atom and direction, $j=1 \ldots 3 N$. Since, for a single normal mode $i$, all atoms oscillate in phase, the real, time-dependent displacements $\vec{A}_{i}$ can be written as

$$
\overrightarrow{A_{i}}=Q_{i} \vec{q}_{i}
$$

where $Q_{i}$ is a the scalar normal mode amplitude which oscillates with the vibration's frequency $\omega_{i}$. The molecular vibration along a normal mode $i$ causes a change in the polarisability of the molecule. Assuming the polarisability to be written as a function of normal coordinates $\vec{q}$, and the departures from equilibrium $Q_{i}$ to be small, one can conveniently formalise 
A
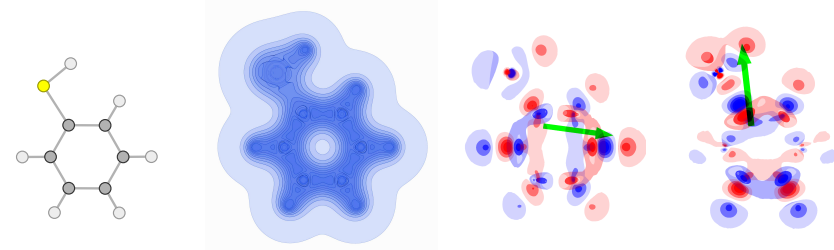

B
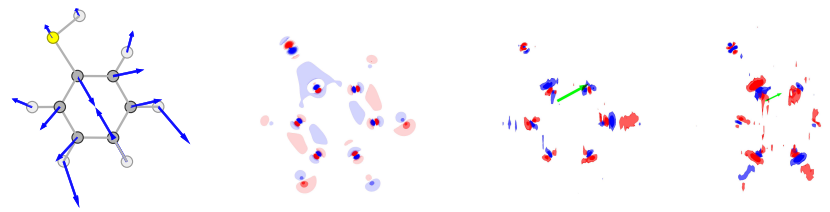

C
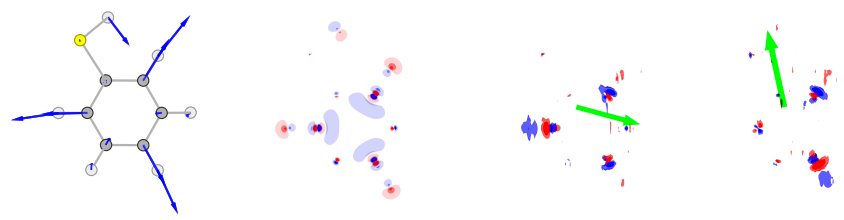

D
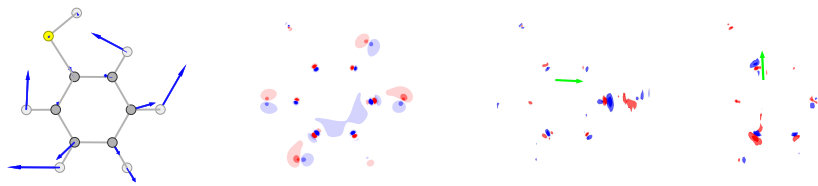

E
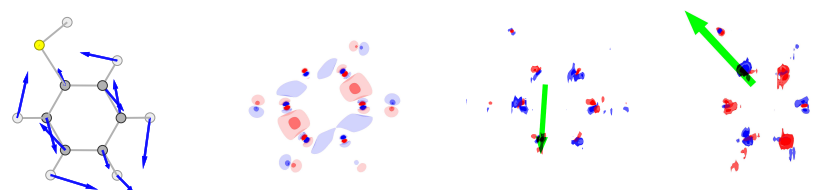

Figure 2.6 - Breakdown of the phenomenological causes of Rayleigh and Raman scattering, using benzenethiol as example. Shown are the molecular structure and vibrational displacements (first column), electron density isosurfaces (second column; calculated with DFT, see chapter 6), difference electron density isosurfaces for a field along the $x$-axis (third column) and the same for a field along the $y$-axis (forth column). The last two columns also show resulting dipole moments (green arrows). The top row pertains to Rayleigh scattering; the vibrationally unperturbed molecule, only affected by the applied electric fields. All other rows show, for all plotted properties, the difference with respect to the top row. This results in Raman dipoles (green) in columns three and four. 
the impact of the vibration as a Taylor series:

$$
\hat{\alpha}=\hat{\alpha}^{0}+\sum_{i}\left(\frac{\partial \hat{\alpha}}{\partial Q_{i}}\right)_{\vec{Q}_{i}=0} Q_{i}+\frac{1}{2} \sum_{i, j}\left(\frac{\partial^{2} \hat{\alpha}}{\partial Q_{i} \partial Q_{j}}\right)_{Q_{i}, Q_{j}=0} Q_{i} Q_{j}+\ldots
$$

Here, the first term, $\hat{\alpha}^{0}$, is the unperturbed Rayleigh polarisability tensor. In the second term, the derivation with respect to the normal mode introduces the Raman tensor:

$$
\hat{R}_{i}=\left(\frac{\partial \hat{\alpha}}{\partial Q_{i}}\right)_{Q_{i}=0}
$$

Higher derivatives of $\hat{\alpha}$ with respect to $\vec{Q}^{\prime}$ s lead to Raman overtones ${ }^{6}$. The magnitude of the tensorial elements decreases rapidly with each term, hence, Rayleigh scattering is much stronger than Raman scattering, which is much stronger than its overtones, etc...

It is principally true that the molecular distortion along a normal mode introduces a change in the electron density, and thus in its polarisability (unless the changes cancel out, depending on the symmetry of the molecule and the normal mode). The effect of this is, however, only observable if the thus perturbed molecule is also perturbed by an em field. In this context, the quantum mechanical nature of the effect becomes evident: assume a photon which is about to excite a vibration via a Stokes process, and the vibrational population of that mode is zero; how can a photon interact with the vibrationally perturbed molecule if the vibration is not present? The answer lies in the quantum nature of the vibration: as a quantum (harmonic) oscillator, each normal mode is always present through its zero point energy, even if unpopulated. Hence, the photon interacts with a molecule which is vibrationally perturbed by its zero point energy.

In Eq. 2.5 the vibration-aware polarisability is a sum, hence, applying the field, the resulting dipole may be expressed as such, with Raman

\footnotetext{
${ }^{6}$ This second-order contribution is usually too small to be observed, except under SERS conditions, where the enhancement of the effective scattering cross section allows for the detection of overtones [19].
} 
components $^{7}$ :

$$
\overrightarrow{p_{\mathrm{R}}^{i}}=\frac{1}{2} Q_{i} \hat{R}_{i} \vec{E}
$$

This context is visualised in Fig. 2.6, with benzenethiol as the exemplary molecule. The top row (A) refers to the vibrationally unperturbed interaction, i.e. Rayleigh scattering, from left to right:

(i) the structure of the molecule as ball-stick model,

(ii) isosurfaces of the electron density (obtained from DFT, see chapter 6),

(iii) the difference electron density after perturbation by an electric field aligned along the x-axis (blue or red colour refer to an increase or decrease in the electronic density) with resulting dipoles (green arrows),

(iv) the same as (iii), but with the field aligned along the y-axis.

The latter two plots hence pertain to Rayleigh scattering. On the second row (B), the influence of the vibrational perturbation onto the molecule are shown, i.e. the plots here show the differences to the plots in the top row:

(i) the structure of the molecule and displacement vectors of the vibration,

(ii) the difference electron density between the vibrationally unperturbed and perturbed molecule,

(iii) the difference electron density between the vibrationally unperturbed and perturbed molecule, with an electric field along $x$. The resulting dipole is the Raman dipole.

(iv) the same as (iii), but with the field aligned along the y-axis.

\footnotetext{
${ }^{7}$ The factor of 2 comes from the time dependence of the normal mode: $Q_{i}(t)=$ $\operatorname{Re}\left[Q_{i}^{0} \exp \left(-i \omega_{i} t\right)\right]=\frac{1}{2} Q_{i}^{0}\left(\exp \left(-i \omega_{i} t\right)+\exp \left(i \omega_{i} t\right)\right)$.
} 
This latter pattern is repeated for other exemplary vibrations in rows (C)(E). Hence, the spectrocopic observables are in the 3rd and 4th columns: Rayleigh dipoles in the top row, and Raman dipoles in the rows below. The tensorial character of the polarisability and its vibrational derivative is evident here: the resulting dipoles are in general not aligned with the field direction, and differ greatly in magnitude. Note that dipole magnitudes and electron density differences were rescaled with respect to the Rayleigh case for clarity. Also note that some Raman dipoles are very weak; this does not necessarily mean that the polarisability derivative is small under these vibrational perturbations. More accurately, it means that the corresponding Raman tensor has only small components along the field directions probed here.

The electronic distortion phenomenologically explains the presence of scattering dipoles. By explicitly including the time dependence of the em field and the vibration, Eq. 2.7 yields:

$$
\begin{aligned}
\vec{p}_{\mathrm{R}}^{i}= & \hat{R}_{i} Q_{i} \operatorname{Re}\left[\exp \left(-i \omega_{i} t\right) \overrightarrow{E_{0}} \exp \left(-i \omega_{L} t\right)\right] \\
= & \frac{1}{2} \hat{R}_{i} Q_{i} \overrightarrow{E_{0}} \operatorname{Re}\left[\exp \left(-i\left(\omega_{L}-\omega_{i}\right) t\right)\right] \\
& +\frac{1}{2} \hat{R}_{i} Q_{i} \overrightarrow{E_{0}} \operatorname{Re}\left[\exp \left(-i\left(\omega_{L}+\omega_{i}\right) t\right)\right],
\end{aligned}
$$

where $\omega_{i}$ is the frequency of the vibration. The dipole radiates at the frequency $\left(\omega_{L}-\omega_{i}\right)$, i.e. is Stokes shifted; or at $\left(\omega_{L}+\omega_{i}\right)$, i.e. is anti-Stokes shifted.

\section{Quantum mechanical approach}

As the above considerations have shown, it is not the polarisability, but the change in polarisability that holds responsible for the Raman effect. As stated already, the presence of a perturbed state prior to the scattering can not be described classically - it requires time-dependent higher-order perturbation theory. The full derivation is beyond the scope of this introduction, which will be limited to the discussion of the results: The 
quantum-mechanical analogue to Eq. 2.5 is

$$
\int \chi_{n_{a}, v^{\prime}}^{*} \alpha \chi_{n_{a}, v}=\alpha_{0} \delta_{v v^{\prime}}+\sum_{i} \int \chi_{n_{a}, v_{i}^{\prime}}^{*}\left(\frac{\partial \alpha}{\partial R_{i}}\right)_{0} R_{i} \chi_{n_{a}, v_{i}} d V_{\text {atoms }}+\ldots
$$

where $n_{a}$ are the quantum numbers of the electronic ground state, $v$ is the vibrational quantum number, therefore $\chi_{n_{a}, v}$ is the corresponding wave function, $R_{i}$ is the $\mathrm{i}^{\text {th }}$ normal coordinate and $d V_{\text {atoms }}$ means integration over the normal coordinates for all atoms. In Eq. 2.9 the first term is Rayleigh scattering again, since $\alpha_{0} \delta_{v v^{\prime}}$ is non-zero only for $v=v^{\prime}$. The integral in the second term can be reduced to a single vibration,

$$
\left(\frac{\partial \alpha}{\partial R_{i}}\right)_{0}=\int \chi_{n_{a} v_{i}^{\prime}}^{*}\left(R_{i}\right) R_{i} \chi_{n_{a}, v_{i}}\left(R_{i}\right) d V_{i}
$$

which is due to the possibility to separate $\chi(\mathbf{R})$ into $\chi\left(R_{1}\right) \chi\left(R_{2}\right) \ldots \chi\left(R_{M}\right)$ and exploit the orthogonality of these functions. When substituting $\chi_{q_{a} v_{i}}$ with the wave function of a quantum-mechanical oscillator, this integral leads to the fundamental Raman transition selection rule:

$$
v_{i}^{\prime}=v \pm 1 \quad .
$$

Therefore this formalism describes vibration creation (Stokes) and destruction (anti-Stokes). When applying the same considerations to the third perturbation term (not shown in Eq. 2.9) one obtains two possible transitions,

$$
v_{i}^{\prime}=v \pm 2 \quad \text { and } \quad v_{i}^{\prime}=0 \quad,
$$

thus accounting for either 1st overtone-generation or no Raman effect at all (annihilation). As in the the classical description, the magnitude of the perturbation terms in Eq. 2.9 decreases rapidly with increasing order of the term.

\subsection{Experimental setup and materials}

Before launching into the more intricate aspects of SERS, a short digression on the experimental background is required to lay the base of the later discussion. 


\section{Spectrometer}

All Raman/SERS spectra shown in this thesis were measured with a Jobin Yvon confocal Raman spectrometer (LabRam) coupled to a liquid $\mathrm{N}_{2}$ cooled CCD detector and a confocal (Olympus BX2) microscope. A photo of the setup is shown in Fig. 2.7, and a schematic of the basic optical setup is provided in Fig. 2.8.

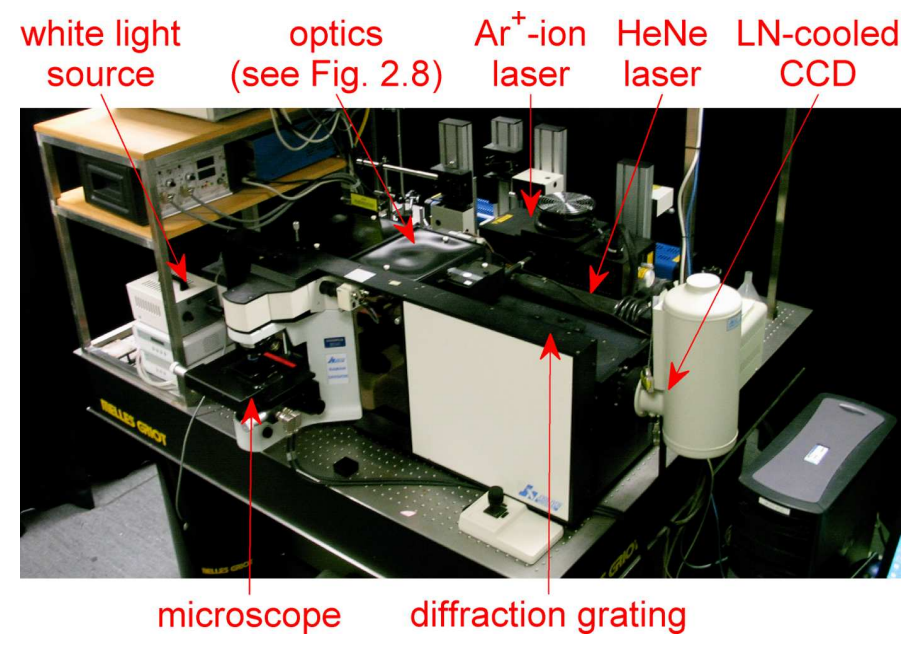

Figure 2.7 — Photo of the LabRam/Olympus setup.

Dry and liquid samples were characterised using a range of objectives: air objectives with magnifications $\times 10, \times 20, \times 50$ and $\times 100$ and an indexmatched (to water) immersion objective, $\times 100(N A=1)$.

For laser excitation the following lasers/lines were used ${ }^{8}$ :

- $\mathrm{Ar}^{+}$-ion laser: $458 \mathrm{~nm}, 514 \mathrm{~nm}(<10 \mathrm{~mW}$ at the sample).

- HeNe laser: $633 \mathrm{~nm}$ ( $3 \mathrm{~mW}$ at the sample).

- diode IR laser: $785 \mathrm{~nm}(<1 \mathrm{~mW}$ at the sample)

\footnotetext{
${ }^{8}$ All lines are continuous wave modes, no pulsed lasers were used.
} 


\section{Materials}

A wide range of analytes was used throughout the work undertaken for this thesis, however, the experimental and theoretical data discussed is limited to three different molecules (with a few exceptions being mentioned in passing), which therefore deserve a small introduction. The molecules/dyes are shown together with their chemical structure in Table 2.1. The absorption spectrum of the dyes is shown in Fig. 2.9.

\section{Rhodamine 6G}

In all experiments referred to in this thesis, Rhodamine 6G (Rh6G) was used as purchased. It was originally developed as a laser dye for the visible range $-i$ it shows strong absorption in the green $\left(\lambda_{\max }^{a b s} \sim 535 \mathrm{~nm}\right)$ and broadly fluoresces in the green and red region, with high quantum yield. ${ }^{9}$ Rh6G has been widely used in the past for SERS and single-molecule SERS. It shows strong SERS spectra under standard conditions, for a number of reasons:

- In solution Rh6G carries one positive charge and thus adsorbs to the negatively charged Ag colloids (see below). The adsorption is believed to be physisorption, thus non-covalent and easily reversible.

- For excitation wavelength in the visible range, the electronic resonance of Rh6G promotes the SERS cross-section via resonance Raman scattering (RRS; excitation within the absorption band) or via strong pre-resonance effects [21] (when exciting within $100-200 \mathrm{~nm}$ red-shifted from the main absorption; see also Sec. 6.9).

- Rh6G is a relatively large molecule (64 atoms including 4 conjugated ring structures) and thus has an intrinsically larger Raman cross section than, for example, methanol. The absolute Raman cross-section

\footnotetext{
${ }^{9}$ A quantum yield of 0.95 was measured in ethanol, see Ref. [20].
} 


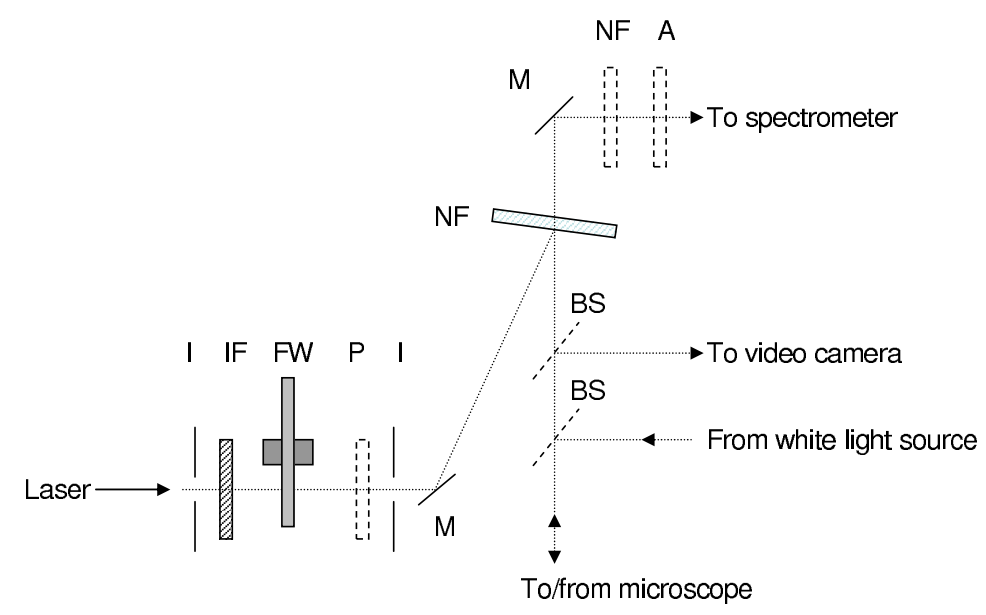

Figure 2.8 - Coupling optics for laser and microscope to the LabRam spectrometer. Components are labelled as follows: I - iris, IF - interference filter, FW - filter wheel (for beam attenuation), P - polariser, A - analyser, M - mirror, NF notch filter, BS - beam splitter. Components drawn with dashed boundaries are optional.

molecule chemical structure

Table 2.1 - Analyte molecules and their chemical structure. 


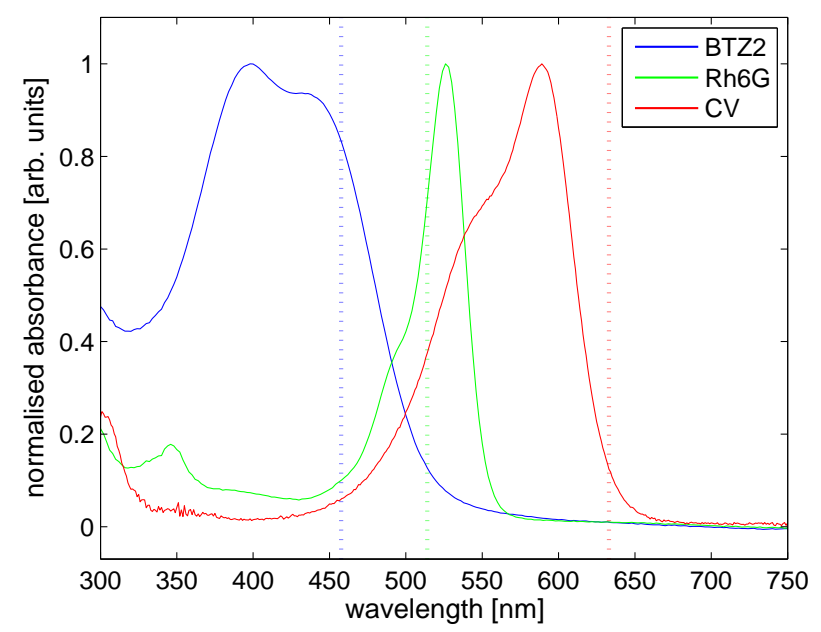

Figure 2.9 - Absorption spectra of common SERS dyes. Shown are BTZ2 (blue), Rh6G (green) and crystal violet (CV, red) as an example for a dye resonant in the red region. The laser lines at $458 \mathrm{~nm}, 514 \mathrm{~nm}$ and $633 \mathrm{~nm}$ are indicated (thick dotted lines). All samples were aqueous solutions with low dye concentrations $\left(c_{\mathrm{BTZ}}=20 \mu \mathrm{M}, c_{\mathrm{Rh} 6 \mathrm{G}}=2 \mu \mathrm{M}\right.$ and $\left.c_{\mathrm{CV}}=4 \mu \mathrm{M}\right)$ - these spectra thus correspond to the absorption of the monomeric moieties. The absorption maxima are located at $389 \mathrm{~nm}$ (BTZ2), $526 \mathrm{~nm}$ (Rh6G) and $589 \mathrm{~nm}$ (CV). 
of Rh6G in water at $633 \mathrm{~nm}$ excitation is $\frac{d \sigma}{d \Omega} \approx 10^{-27} \mathrm{~cm}^{2} \mathrm{sr}^{-1}$ (mode dependent, see Ref. [21]). For comparison, methanol scatters four orders of magnitude less: $\frac{d \sigma}{d \Omega} \approx 10^{-31} \mathrm{~cm}^{2} \mathrm{sr}^{-1}$.

\section{BTZ2}

The benzotriazole dye $\mathrm{BTZ} 2{ }^{10}$ was synthesized following the procedure described in Ref. [22] (dye \# 2 of this reference). The yellow aqueous solution shows broad absorption below $500 \mathrm{~nm}$. Due to its tri-azo group the dye is believed to strongly adsorb (covalent bonding) to the silver surface [22]. BTZ2 was specifically designed for SERS studies, and accordingly shows large signals. Its bare Raman cross-section at $633 \mathrm{~nm}$ is $\frac{d \sigma}{d \Omega} \approx 10^{-28} \mathrm{~cm}^{2} \mathrm{sr}^{-1}$ (mode dependent, see Ref. [21]).

\section{SERS Substrates}

Two principally different types of substrates were used: liquid and dry. The base for both is negatively charged Ag-colloids which were prepared according to the standard procedure described by Lee \& Meisel (see Ref. [23] and chapter 4). I will describe the specific sample preparations where appropriate later in text, but the general recipe can be outlined here:

1. start with bare Lee \& Meisel colloid solution,

2. add dye to desired concentration ${ }^{11}$, usually $<1 \mu \mathrm{M}$, and let the dye adsorb for a few minutes,

3. add $\mathrm{KCl}$ to a final concentration of $10 \mathrm{mM}$ and incubate for $\sim 10 \mathrm{~min}$ (see Section 4.4),

4. optionally, dry the final sample onto a glass or silicone slice.

\footnotetext{
${ }^{10}$ Full name: 3-methoxy-4-(5'-azobenzotriazolyl)phenylamine

${ }^{11}$ Note that throughout the entirety of this thesis, unless stated otherwise, concentrations are given in the commonly used molarity $\mathrm{M}$, which is defined as $M=10^{-3} \mathrm{~mol} / \mathrm{m}^{3}$.
} 
A more thorough characterisation of the colloidal sol is the topic of chapter 4 , which also describes the procedure to prepare dry samples in full.

This shall complete the brief excursion on the experimental details. Together with the theory of the Raman effect the stage has now been set to conceptually introduce the surface-enhancement mechanism, i.e. the effect of the surface-proximity on molecular Raman scattering. 


\section{Chapter 3}

\section{Surface-enhancement mechanisms}

[...] a straight line law connecting any empirical data always can be achieved with the aid of suitably scaled logarithmic paper and a robust conscience.

Robert A. Fairthorne

\subsection{Electromagnetic surface enhancement}

The surface enhancement achieved through re-distribution of electromagnetic fields accounts for the bulk of the enhancement observed in SERS [24, 25, 21]. Though other aspects (e.g. molecule-surface charge-transfer complexes, molecular orientation; i.e. the 'chemical' enhancement) may play a role, their influence on the observed intensity is generally much weaker ${ }^{1}$. In a nutshell, the SERS substrate interacts with the em field and re-distributes it, usually resulting in strongly localised regions of high field intensities - so called hotspots. A molecule which resides in a hotspot is driven by the am-

\footnotetext{
${ }^{1}$ For a discussion see Section IV in Moskovits' 1985 review, Ref. [25]
} 
plified local field, and, moreover, the self-reaction of the molecular dipole with its environment modifies the dipolar emission [17].

Due to the complexity of the topic, in what follows, the discussion is inevitably limited to cover only the most salient aspects of the underlying physical principles, however, due to its relevance for the model SERS hotspot, I will cover the model of the dimer of Ag spheres (see Sec. 3.1.5) in more depth. For an exhaustive discussion of all the topics presented in this chapter the reader is referred to Le Ru and Etchegoin's book [17], chapters 3-6 and appendices $\mathrm{C}-\mathrm{H}$.

The layout of the chapter is as follows: Sec. 3.1.1 gives a brief primer on SERS enhancement factors and includes some definitions necessary in the later discussion; Sec. 3.1.2 lays out the physical foundation of SERS by introducing the dielectric function of noble metals; Sec. 3.1.3 surveys the most common approaches taken to solve Maxwell's equations in systems with more or less complex geometric structures and material properties; in Sec. 3.1.4, the basic example of a planar surface is used to introduce some important concepts in SERS. Equipped with these preliminaries, and concluding the scope of this chapter, the concept of the SERS hotspot is then studied in Sec. 3.1.5.

\subsubsection{Enhancement factors}

A common phrase in the SERS literature is "... the signal is enhanced by a factor of ...", or a variation thereof. Irrefutably, the SERS enhancement factor (EF) is one of the most important parameters in the assessment of the strength of the effect. Indeed, it appears that the EF has evolved into much of a label advertising the grandeur of SERS, with authors seemingly striving to post the highest conceivable numbers. EFs up to $10^{14}$ are commonly quoted [8, $26,27,28]$, despite being in disconcert with theoretical estimates [29, 30] and experiments $[31,21]$, which consistently put the bar around $10^{10}-10^{11}$. The more disheartening it is then to realise that only few in the field [31,21] 
properly define and measure the EF they quote. Correspondingly, the lack of proper taxonomy made SERS EFs subject to a Babylonian confusion of tongues, leading to disagreement about the magnitude and origin of the enhancement. As Moskovits observed ${ }^{2}$, the apparent disagreement in EFs persists simply because "there is no one enhancement factor". In fact, one can define a whole plethora of EFs [21,17] without leaving the realm of experimental significance. To this end, it shall suffice to enumerate, but not elaborate upon, the various EFs. The only EF used quantitatively later in this thesis is the $|\vec{E}|^{4}$-approximation, which is made plausible below ${ }^{3}$.

\section{$|\vec{E}|^{4}$-approximation}

As stated before, the surface enhancement is above all an em effect; after all, single molecules (see chapter 5) cannot be seen on flat surfaces, where a chemical enhancement (see Sec. 3.2) would already be present. The incident light interacts with the electronic structure of the substrate (mostly free electrons in case of a noble metal like Ag), leading to surface plasmon resonances (SPR). At these localised resonances the incident $\mathrm{em}$ fields are amplified, and -vice versa- the em field of the scattered radiation is amplified as well. In this context it is common to define a field enhancement factor which compares the local field intensity $\left|\vec{E}_{\text {loc }}\right|^{2}$ with the non-enhanced field $\left|\vec{E}_{0}\right|^{2}$. Since intensities are measured, the enhancement of the incident radiation $M_{\text {in }}$ is expressed as:

$$
M_{\mathrm{in}}(\lambda)=\frac{\left|\vec{E}_{\mathrm{loc}}(\lambda)\right|^{2}}{\left|\vec{E}_{0}(\lambda)\right|^{2}} .
$$

Solving the em problem (see Sec. 3.1.3, ff.) directly yields this enhancement. The local field is what is 'felt' by a molecule at the surface, therefore, by

\footnotetext{
${ }^{2}$ in a private conversation at the Colloquium Spectroscopicum Internationale $\mathrm{XXXV}$, Xiamen 2007

${ }^{3}$ For a more rigorous treatment, see Ref. [32]
} 
analogy, this is equivalent to a situation in which a laser with $M_{\text {in }}$-fold power irradiates a free molecule. This analogy holds for the excitationpart, however, the radiation of the thus driven Raman-dipole is enhanced by its self-reaction in the presence of the SERS substrate. Since this effect is difficult to quantify (it requires solving the problem of dipolar emission, which is substantially harder than dealing with incident waves), the radiation enhancement $M_{\text {out }}$ is commonly (and conveniently) assumed to be the same as $M_{\mathrm{in}}$. The overall enhancement thus writes:

$$
M\left(\lambda, \lambda^{\prime}\right)=M_{\text {in }}(\lambda) M_{\text {out }}\left(\lambda^{\prime}\right)=\frac{\left|\vec{E}_{\mathrm{loc}}(\lambda)\right|^{2}}{\left|\vec{E}_{0}(\lambda)\right|^{2}} \frac{\left|\vec{E}_{\mathrm{loc}}\left(\lambda^{\prime}\right)\right|^{2}}{\left|\vec{E}_{0}\left(\lambda^{\prime}\right)\right|^{2}} .
$$

Note that the enhancement depends on the incident and the Raman-shifted wavelengths $\lambda$ and $\lambda^{\prime}$ - as can be calculated (e.g. by Mie-Theory [33, 34], see Sec. 3.1.5), the intensity of the plasmonic resonance varies with wavelength. Therefore, for each vibrational mode $i$ in a molecule one observes a principally different enhancement $M\left(\lambda, \lambda_{i}\right)$. It is important to note that this is not an intrinsic mode-dependence, but a wavelengthdependence, as the enhancements for Stokes and anti-Stokes shifted peaks differ as well. One can simplify Eq. 3.2 further by neglecting the Raman shift, i.e. setting $\lambda=\lambda^{\prime}$ :

$$
M(\lambda)=\frac{\left|\vec{E}_{\mathrm{loc}}(\lambda)\right|^{4}}{\left|\vec{E}_{0}(\lambda)\right|^{4}} .
$$

This is the EF in the $|\vec{E}|^{4}$-approximation. It was reported already in one of the first SERS papers [5]. It is important to bear in mind the crude character of this approximation; it is little more than a yardstick measure from which one can draw some qualitative conclusions [32].

As an example in which this simple approach clearly fails to yield physically meaningful enhancement factors, one can consider the extreme case of a molecule with a uniaxial Raman tensor being subjected to a field polarised perpendicular to it, i.e. $\hat{\alpha} \vec{E}=0$. The (fixed) molecule in free-space 
then is not expected to interact with the field at all. However, the local field in general does not retain the incident polarisation. In the local environment the molecule hence would interact with light. Comparing this with the free-space molecule, one would arrive at an infinite enhancement. Conversely, one can expect the $|\vec{E}|^{4}$-approximation to work well within its scope (yardstick measure) in the case of isotropic Raman tensors and low Raman shifts.

Accordingly it emerges that, depending on the desired assessment, EFs need to be tailored to the experimental or theoretical specifics.

\section{Other EFs}

It is worth noting that simplicity, experimental feasibility and hence reproducibility are inversely proportional to the amount of physical detail accounted for in EF definitions. One can quickly and rigorously define an enhancement factor with little experimental use.

On the simple end, an intuitively understandable EF is the analytical enhancement factor (AEF). It simply answers the question: "Compared to normal Raman scattering (RS), by what factor can one decrease the analyte concentration, yet still yield the same Raman intensity?" It is formally defined as:

$$
\mathrm{AEF}=\frac{I_{\text {SERS }} / c_{\text {SERS }}}{I_{\mathrm{RS}} / c_{\mathrm{RS}}},
$$

where $I$ is the scattered intensity and $c$ is the analyte concentration. The fact that, under SERS conditions, the molecules will (ideally) be absorbed onto the substrate, is swept under the carpet in this definition. Hence, the AEF will not only vary across substrates, but also across analytes, since it ignores surface adsorption efficiency - it is thus not suited to characterise a SERS substrate for any analyte. On the other hand, it is well suited (given a reference AEF) to assess the SERS-activity of liquid substrates (colloidal sols) with respect to one particular analyte.

Taking account of the surface adsorption, the SERS substrate EF (SSEF) 
normalises intensities to the actual number of molecules partaking in the scattering event:

$$
\mathrm{SSEF}=\frac{I_{\text {SERS }} / N_{\text {surface }}}{I_{\text {RS }} / N_{\text {volume }}}
$$

The SSEF characterises the average enhancement of a substrate [35]. However, notably, determining $N_{\text {surface }}$ poses a significant step-up in experimental hardship.

The single-molecule enhancement factor (SMEF) is designed to accurately portray the enhancement felt by a single molecule - it requires proper determination of the (effective) cross-sections under normal Raman and SERS conditions ${ }^{4}$.

$$
\mathrm{SMEF}=\frac{d \sigma_{\text {SERS }} / d \Omega}{d \sigma_{\text {Raman }} / d \Omega}
$$

Without going into further detail, I shall mention in passing the mere existence of EF definitions which, in addition to the effects elaborated upon above, take into account selections of the following properties:

- incident polarisation

- molecular orientation

- scattering configuration

- the Raman tensor of the specific mode

An exhaustive review on EFs is given in Ref. [21] (also see the supporting information) and in chapter 4 of Ref. [17].

\subsubsection{Dielectric function of noble metals}

Ultimately, the physical reason why $\mathrm{Au}$ and $\mathrm{Ag}$ are so heavily used for SERS stems from the fact that noble metals feature free conduction elec-

\footnotetext{
${ }^{4}$ This is experimentally cumbersome since it requires knowledge on the scattering volume, etc... Also, the bare Raman signal of dyes is often buried in fluorescence, hence not measurable with sufficient accuracy.
} 
trons. Since these electrons are unbound, they are -in principle- free to interact with $\mathrm{em}$ fields. One can picture this as: (i) the incident wave driving the electrons back-and-forth along its oscillating $\vec{E}$-field (and projected onto the electrons plane of mobility, i.e. the metallic surface) and (ii) the driven electrons thus turning into, if damping is negligible, dipoles which emit the reflected wave at the very same frequency. This strong coupling of conduction electrons to the em field is the basis for the field re-distribution on a substrate. The resulting em enhancement, which shall be touched later (see Sec: 3.1.4 and 3.1.5), then has a strong geometrical dependence (e.g. the field distribution and enhancement on a sphere is different from a plane).

\section{The Drude model}

The Drude model stems from the Lorentz model of atomic polarisability, which assumes the electrons to be classical harmonic oscillators - oscillating around fixed atomic cores, i.e. non-free electrons. In this model, the interaction with the field is then described by the equation of motion of a classical, forced, dampened harmonic oscillator:

$$
m\left(\frac{\mathrm{d}^{2} \vec{r}}{\mathrm{~d} t^{2}}+\omega_{0}^{2} \vec{r}+\Gamma \frac{\mathrm{d} \vec{r}}{\mathrm{~d} t}\right)=-e \vec{E}
$$

where $\vec{r}$ is the electron's displacement, $\omega_{0}$ is the natural frequency of oscillation, $\Gamma$ contains the damping the electrons experience within their environment and $e$ and $m$ pertain to the effective charge and mass of the electron(s). By convention, $\vec{E}$ is defined as a harmonically oscillating field via:

$$
\vec{E}=\operatorname{Re}\left[\overrightarrow{E_{0}} \exp (-i \omega t)\right]
$$

Eq. 3.7 is then satisfied by:

$$
\vec{r}=\frac{-e / m}{\omega_{0}^{2}-\omega^{2}-i \Gamma \omega} \vec{E}
$$


This leads to the atomic dipole moment $\vec{p}=-e \vec{r}$, from which, with $\vec{p}=\alpha \vec{E}$, one can express the optical polarisability $\alpha$ as:

$$
\alpha=\frac{e^{2} / m}{\omega_{0}^{2}-\omega^{2}-i \Gamma \omega}
$$

Given the electron density $n$, one can extrapolate to the macroscopic polarization: $P=n \alpha \vec{E}$. This yields the connection to the dielectric function $\varepsilon$ since $P=\chi \varepsilon_{0} \vec{E}=(\varepsilon-1) \varepsilon_{0} \vec{E}$. Thus, from the atomic polarisability (Eq. 3.10) the macroscopic dielectric function is obtained:

$$
\varepsilon(\omega)=1+\frac{n \alpha(\omega)}{\varepsilon_{0}}=1+\frac{n e^{2}}{m \varepsilon_{0}} \frac{1}{\left(\omega_{0}^{2}-\omega^{2}-i \Gamma \omega\right)}
$$
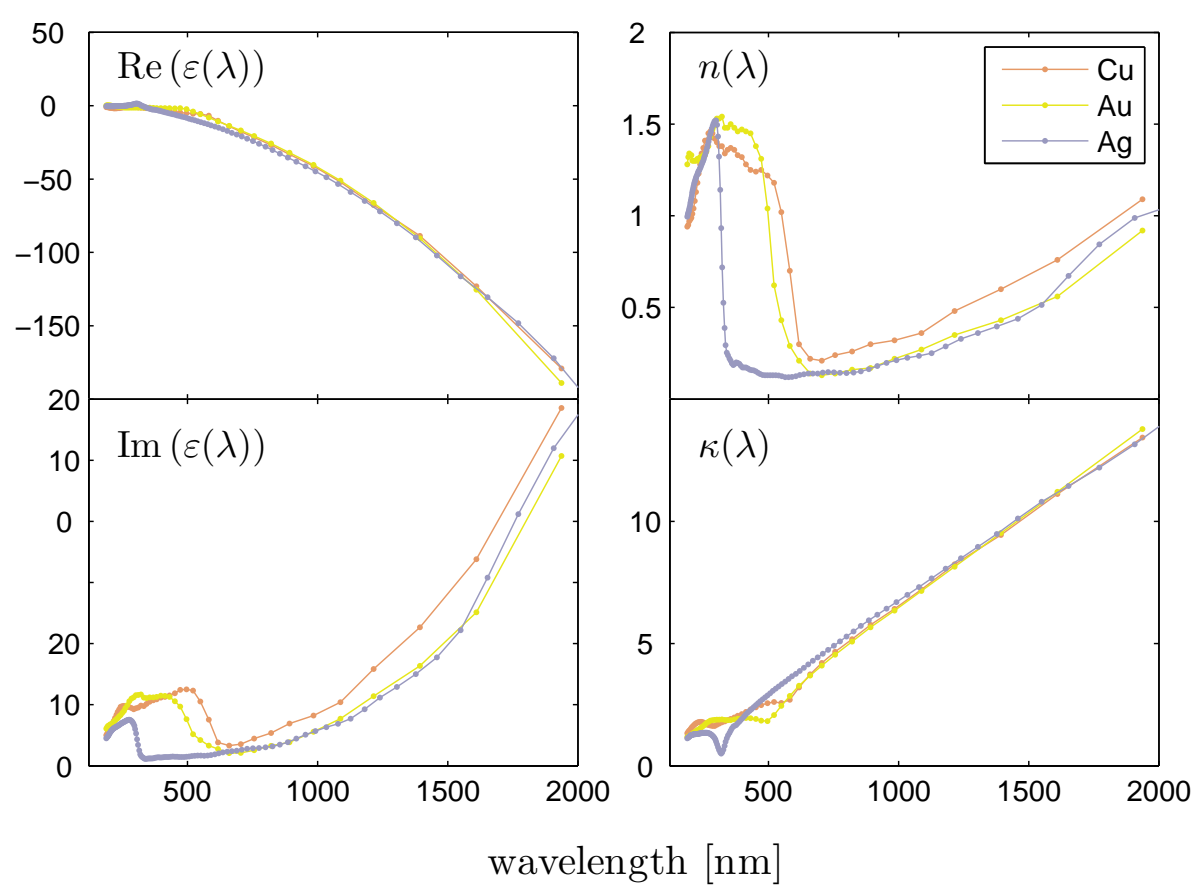

Figure 3.1 - Optical properties of typical SERS substrate elements copper, gold and silver (see legend). Shown are the real part (top left) and imaginary part (bottom left) of the dielectric function and, complementary, the index of refraction $n$ (top right) and extinction coefficient $\mathcal{k}$ (bottom right). Copper and gold data was taken from Johnson \& Christy [36] and silver from Palik [37].

This is as far as the Lorentz model reaches. Based on these foundations, the Drude model implements modifications by 
- assuming free electrons, i.e. $\omega_{0} \rightarrow 0$, which simplifies the denominator in Eq. 3.11,

- approximating the optical response of the positive ion grid of the metal as a constant factor, $\varepsilon_{\infty}$.

The resultant dielectric function is:

$$
\varepsilon(\omega)=\varepsilon_{\infty}\left(1-\frac{n e^{2}}{m \varepsilon_{0}} \frac{1}{\omega^{2}+i \Gamma \omega}\right)
$$

Here it is common to introduce the plasma frequency $\omega_{p}$, which is the natural oscillation frequency of the plasma of free electrons:

$$
\omega_{p}=\sqrt{\frac{n e^{2}}{m \varepsilon_{0} \varepsilon_{\infty}}} .
$$

This compacts Eq. 3.12 to:

$$
\varepsilon(\omega)=\varepsilon_{\infty}\left(1-\frac{\omega_{p}^{2}}{\omega^{2}+i \Gamma \omega}\right)
$$

This complex-valued function describes the free-electron contribution to the optical response of metals, and I shall briefly highlight its main aspects here. For the discussion, it is customary to split the function into its real and imaginary parts:

$$
\begin{aligned}
\varepsilon & =\varepsilon^{\prime}+i \varepsilon^{\prime \prime} \\
\varepsilon^{\prime} & =\operatorname{Re}[\varepsilon(\omega)]=\varepsilon_{\infty}\left(1-\frac{\omega_{p}^{2}}{\omega^{2}+\Gamma^{2}}\right) \\
\varepsilon^{\prime \prime} & =\operatorname{Im}[\varepsilon(\omega)]=\frac{\varepsilon_{\infty} \omega_{p}^{2} \Gamma}{\omega\left(\omega^{2}+\Gamma\right)}
\end{aligned}
$$

A connection to optical properties is made by comparing $\tilde{\varepsilon}$ with the complex index of refraction $(\tilde{n}=n+i \kappa)$; both are close relatives, via $\tilde{\varepsilon}=\tilde{n}^{2}$. The components of $\tilde{n}$ are the index of refraction $n$ and the extinction coefficient $\kappa$. The real and imaginary part of $\tilde{\varepsilon}$ thus can be expressed as: $\varepsilon^{\prime}=n^{2}-\kappa^{2}$ and $\varepsilon^{\prime \prime}=2 n \kappa$. 
Figure $3.2-$ (Top) Real part and (bottom) imaginary part of the dielectric function of Ag from the UV to the near IR. The insets expand the visible spectral region mostly used in SERS. Shown are the experimental data by Johnson \& Christy [36] (०), Palik [37] (), Sambles [38] $(\boldsymbol{\Delta})$ and a Drude model fit (solid line; parameters given in Eq. 3.18). Note the Drude-like behaviour throughout the visible range and IR. Absorption sets in for $\lambda<320 \mathrm{~nm}$.

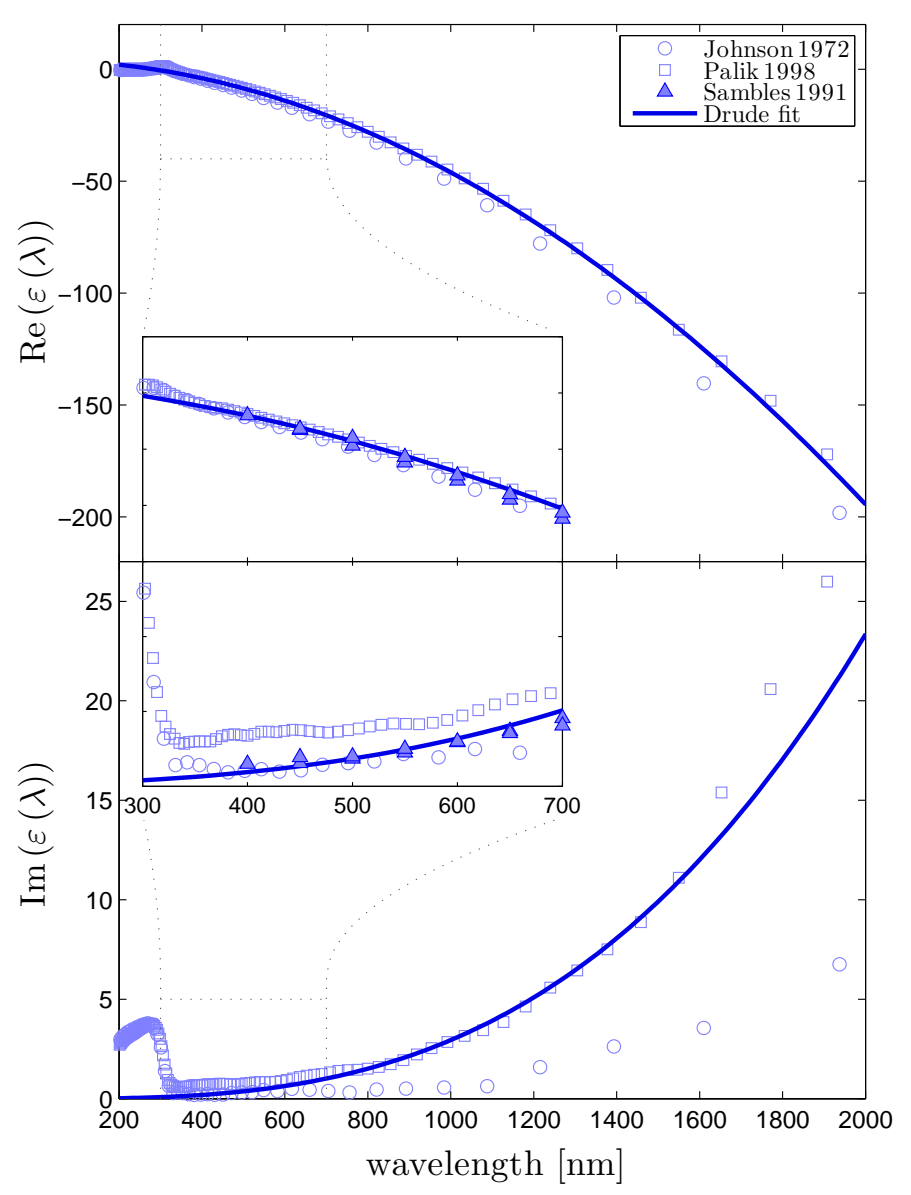

\section{Dielectric function of silver}

Experimental measurements of the dielectric function of silver, and a fit of Eq. 3.14 to these values, are shown in Fig. 3.2. Expressing Eq. 3.14 in wavelengths, the Drude-fit has the following parameters:

$$
\begin{gathered}
\varepsilon_{\mathrm{Ag}}(\lambda)=\varepsilon_{\infty}\left(1-\frac{1}{\lambda_{p}^{2}\left(\frac{1}{\lambda^{2}}+\frac{i}{\mu_{p} \lambda}\right)}\right) \\
\varepsilon_{\infty}=4, \quad \lambda_{p}=282 \mathrm{~nm}, \quad \mu_{p}=17000 \mathrm{~nm}
\end{gathered}
$$

This function is used in the em simulations undertaken in Sec. 3.1.5. Note the discrepancy in the experimental values in the visible range - these stem 
from the different techniques used in the (non-trivial) measurement of $\varepsilon$. Both Palik ( $\square$ in Fig. 3.2) and Johnson \& Christy ( $\circ$ in Fig. 3.2) ignore the presence of plasmons, which, if taken into account (Sambles data [38], $\mathbf{\Delta}$ in Fig. 3.2), provide a better agreement with the Drude fit.

\section{Dielectric function of gold}

Gold was not used as a substrate in the experiments described in this work, but is, nonetheless, one of the classic substrates used for surface-enhanced spectroscopies. Au takes its advantages from its well-understood surface chemistry and known bio-compatibility. To this end, the dielectric function of gold was studied simply since there was, initially, no analytical expression adequately fitting the experimental data. As opposed to Ag, in

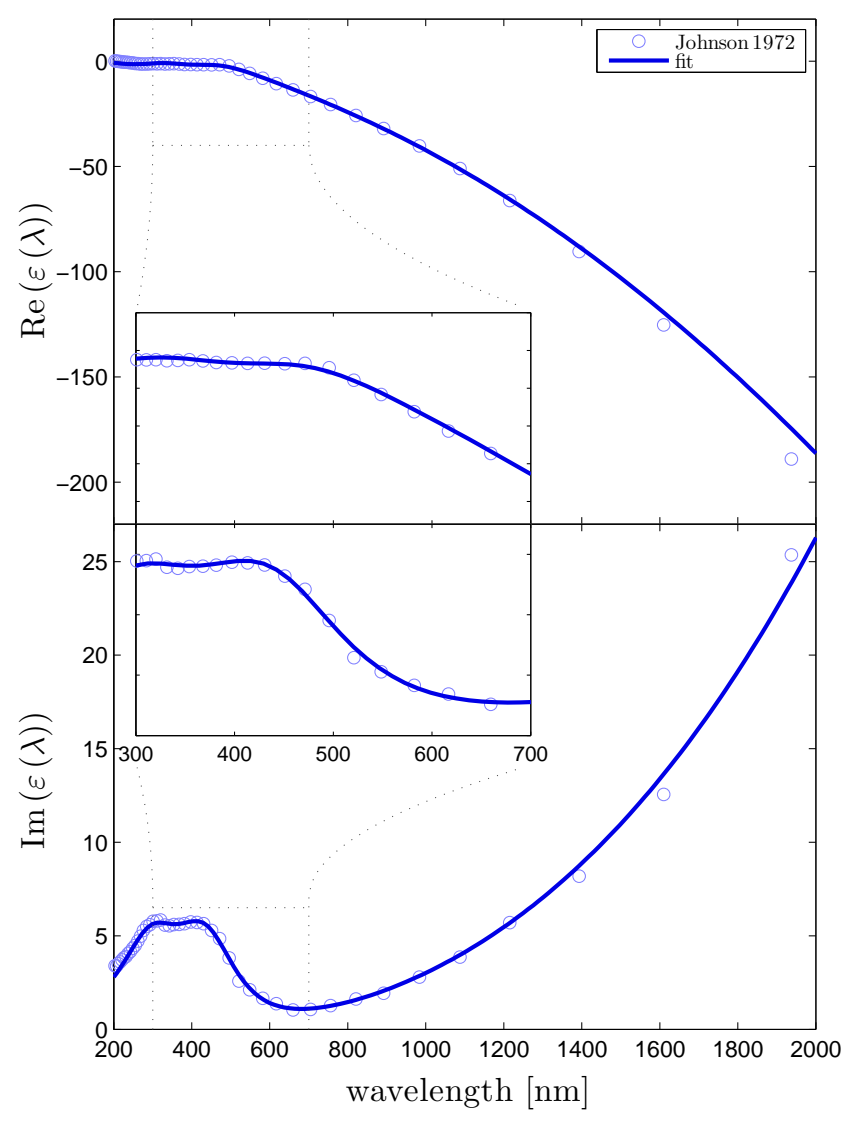

Figure $3.3-$ (Top) Real part and (bottom) imaginary part of the dielectric function of gold from the UV to the near IR. The insets expand the visible spectral region mostly used in SERS. Shown are the experimental data by Johnson \& Christy [36] (०) and the fit by Etchegoin [39, 40] (solid line). See text for details. 
$\mathrm{Au}$ the onset of absorption (caused by intraband transitions) happens in the visible region. While the Drude model fits well in the infra-red region, the lineshape in the visible (i.e. the absorption region) requires additional terms to the dielectric function. These can not be approximated well by a small number of Lorentz-, Gauss- or Voigt-curves. Borrowing the concept of critical points from semiconductor physics [41], Etchegoin et al. managed to cast the fit into a compact formula (which shall be omitted here to avoid undue typographical strain) - the interested reader is referred to Ref. [39] and its erratum [40]. Fig. 3.3 shows the Johnson \& Christy data [36] (०) and the mentioned fit (thick line).

\subsubsection{Solving the electromagnetic problem}

The solution to Maxwell's equations in the SERS context is far from trivial. The stage is set by the substrate: colloidal (or otherwise 'roughened') metal substrates which are -in general- of arbitrary geometry. Solving the em field re-distribution in this scenario requires sophisticated analytical methods or, of more general applicability, numerical algorithms ${ }^{5}$.

\section{Numerical approximate solution via discretisation}

For arbitrary geometries, typically, the problem is discretised into small chunks and, with given boundary conditions, the solution is propagated through the discrete space. Some commonly used methods of this type are:

Discrete dipole approximation - DDA [43, 44, 45], splits the space domain into a set of polarisable points. The solution propagates through the interaction of the resulting dipoles.

\footnotetext{
${ }^{5}$ For a general introduction into "Computational Electromagnetics", see Ref. [42]
} 
Finite-difference time-domain - FDTD $[46,47]$, discretises the space domain and solves time-steps for $\vec{E}$ and $\vec{H}$, by connecting the space and time domains through Maxwell's curl equations.

Finite element modelling - FEM, is a general approach to the solution of partial differential or integral equations. The space domain is, in general, discretised non-uniformly ('meshed') and the problem's solution is approximated by choosing a basis function, which extrapolates the solution within the subdomain of each mesh cell. An in-depth discussion of the application of the finite element strategy to electromagnetic modelling can be found in the books by Monk [48] and Volakis [49].

The list of discretisation-based approaches is long, and it is outside the scope of this thesis to dive any deeper into the specifics of each implementation [50]. What one should take away from here is that: (i) there are many numerical methods, each with their respective advantages and disadvantages and (ii) all of these methods share one principal problem, which is somewhat intrinsic to SERS: the time- and memory-requirements of fine-grained discretisations. Why is this intrinsic to SERS? - because hotspots are generally very small compared to the overall colloid/substrate and field wavelength, but a much larger volume is usually required for accurate results. For example, to model the generic situation of a hotspot in the gap between two colloids, one deals with various length scales; ordered from small to large these are: (i) the hotspot, where field intensities may vary orders of magnitude across a single nanometer, (ii) the colloids, typically with a $\sim 20-100 \mathrm{~nm}$ diameter and (iii) the modelled environment around the colloids, which needs to be large enough to avoid simulation artifacts on boundaries, etc..., typically at least $\sim 1 \mu \mathrm{m}^{3}$. Modeling these length scales appropriately is non-trivial; when using uniform grids, it quickly becomes infeasible. 


\section{Analytic solutions for special geometries}

Analytic solutions to the electromagnetic problem exist for a few special cases, most notably highly symmetric problems: planar, cylindrical and spherical geometries. Of particular relevance for SERS is the case of single or multiple spheres - modelling single colloids and aggregates. Gustav Mie [51] developed an analytical solution for the single sphere - now known as Mie theory $[52,53]$. With a vast increase in complexity, Generalised Mie theory $[54,55,56,17]$ (GMT) extends this ansatz to multiple spheres (including overlapping and 'coated' spheres) and related shapes, i.e. ellipsoids. While the theory in principle provides analytic solutions, in practice the result is a numeric evaluation of the analytic solution. In Sec. 3.1.5, I will discuss in depth the model of a dimer of spheres solved by GMT.

\subsubsection{Local field of a planar surface}

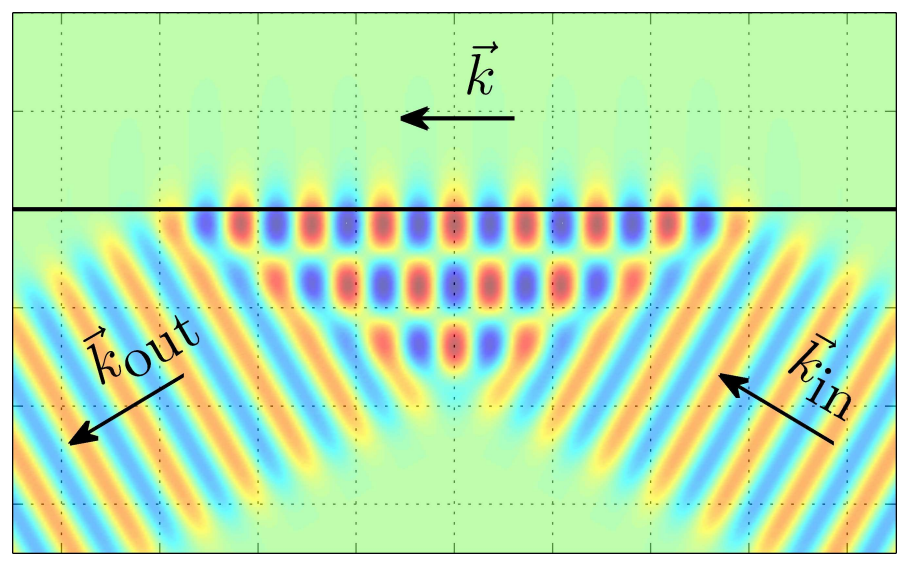

Figure 3.4-Schematic representation (not a strict simulation) of the evanescent field arising from total internal reflection. The incident and reflected field in the medium superimpose at the boundary into a longitudinal wave, i.e. $\vec{E}$ and $\vec{k}$ are both parallel to the surface. Above the boundary, the longitudinal wave spills into space, whereas the field intensity drops exponentially with distance to the surface.

A planar surface is a far abstraction from the conditions in most real-world 
SERS experiments, and, moreover, its interaction with electromagnetic waves is a standard textbook problem [57, 58, 59], mostly treated (when complex indices of refraction are considered) by the Fresnel coefficients [60]. Nonetheless, it is worth the brief digression since the effects encountered already contain a number of aspects important in SERS, and thus, it serves as a didactic device to introduce these concepts:

Polarisation dependence: As should be obvious, transmission and reflection of the incoming wave depends -as exemplified by the Brewster angle- on the polarisation of the $\mathrm{em}$ wave with respect to the surface (TE- and TM-waves, or $s$ - and $p$-polarisation). I mention this here since in more complex (less symmetric) geometries, from the SERS point of view, polarisation is a crucial parameter: For example, the field magnitude in the hotspot of a dimer of colloids strongly depends on the orientation of the dimer with respect to the field polarisation [17, 61, 62]. This has also been confirmed experimentally $[63,28]$.

Evanescent fields: Much like the standing wave solution in a potential well tunnels (with exponentially decreasing amplitude) into the wall, an $\mathrm{em}$ wave confined at a boundary will 'spill' across the interface, with exponentially decreasing amplitude. Consider a TM-wave undergoing total internal reflection (see Fig. 3.4): at the boundary, the incident and the reflected wave sum into a longitudinal wave, with both $\vec{E}$ and wave vector $\vec{k}$ parallel to the surface. Across the boundary, the momentary field decays exponentially into the optically thinner medium, perpendicular to the boundary. The evanescent field moves with the boundary field, i.e. propagates with $\vec{k}$ parallel to the boundary.

Surface modes and $\vec{k}$-conservation: Given the skin-effect, how can an em wave propagate in a perfect metal? One of the conditions is that the plasmon's $\vec{k}$ needs to be parallel to the surface, however, then it is impossible to excite this plasmon by an incident wave, since the 
$\sum \vec{k}$ would not be conserved. With the aid of evanescent fields the $\vec{k}$-conservation criterion is overcome - it is possible to excite propagating plasmons (strictly, propagating surface plasmon-polaritons [17]) in a planar surface. This is experimentally achieved in the Otto and Kretschmann [64] configurations. The momentum mismatch can also be overcome by patterning the surface, or, more generally, by roughening it $^{6}$.

Surface selection rules: The radiation of dipoles near the surface can be enhanced or quenched depending on the relative orientation with respect to the surface. This orientation dependence becomes obvious when invoking the method of image charges (see Fig. 3.5), which describes the metal's reaction in the electrostatic limit. Furthermore, in the long-wavelength limit, it is easy to see that the field component parallel to the surface vanishes (since the interface is a conductor), while the perpendicular component is enhanced (superposition of incident and reflected wave). Both effects, the self-reaction of the dipole and the modified local field, lead to a selective enhancement of Raman modes.

I will now depart from this brief digression on the planar surface and refocus onto a more SERS-specific model: the dimer of spheres. From the didactic point of view, this poses a discontinuity since the discussion of a dimer of spheres should be predated by a treatise of the single sphere. Nonetheless, I will omit this step since the single sphere, although interesting from the theoretical point of view, does not add to the concepts significant for this short discussion.

\footnotetext{
${ }^{6}$ Which is how SERS was first discovered: on electrochemically roughened silver, see Ref [4].
} 

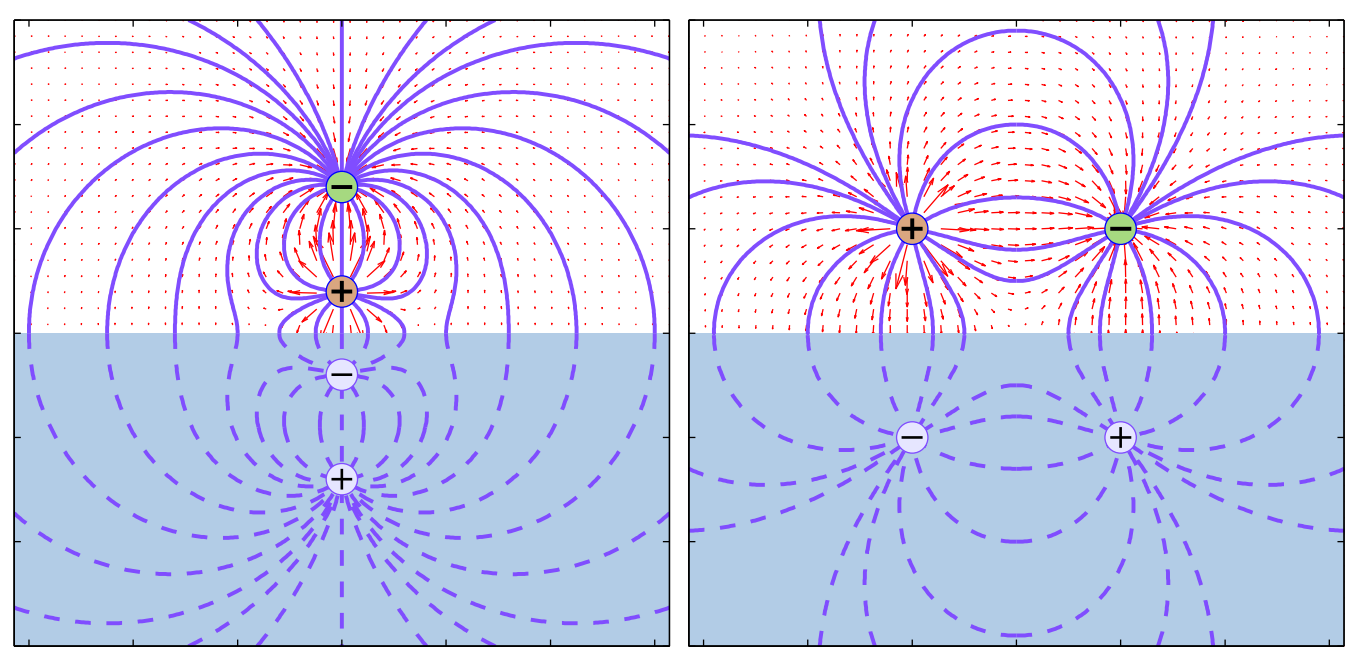

Figure 3.5 - Concept of image dipoles: electrostatic dipole oriented perpendicular (left) and parallel (right) to a metal surface, together with the (virtual) image dipole. Shown are electric field vectors (red), field lines (blue) and the metal (light blue). In the perpendicular orientation (left), the presence of the image dipole increases the dipolar field strength outside the metal, i.e. the two dipoles combine constructively. In the parallel case (right), the real and image dipole 'cancel', strongly reducing the dipolar character of the field outside the metal; a quadrupolar field results. Note that the field lines drawn here are those produced by the dipoles (image dipole explicitly included) - the metal is not taken into account, the boundary conditions appear to be matched here solely due to the symmetry of the mirror dipoles. Image dipoles are virtual, i.e. the real field outside the metal is as if an image dipole were present, however, the real charge distribution and field within the metal looks nothing like it: in the electrostatic limit it is only concentrated on the surface, otherwise it has a small penetration depth.

\subsubsection{Local field of a dimer of spheres}

The dimer of spheres, i.e. two spheres separated by a small gap (see Fig. 3.6), is a standard model system mimicking colloidal SERS. The em problem is analytically solved by Generalised Mie-Theory, which yields near-field (the local field at and close to the metallic surface) and far-field properties (extinction, absorption and scattering coefficients). The local field harbours the conceptually most critical feature for the understanding 

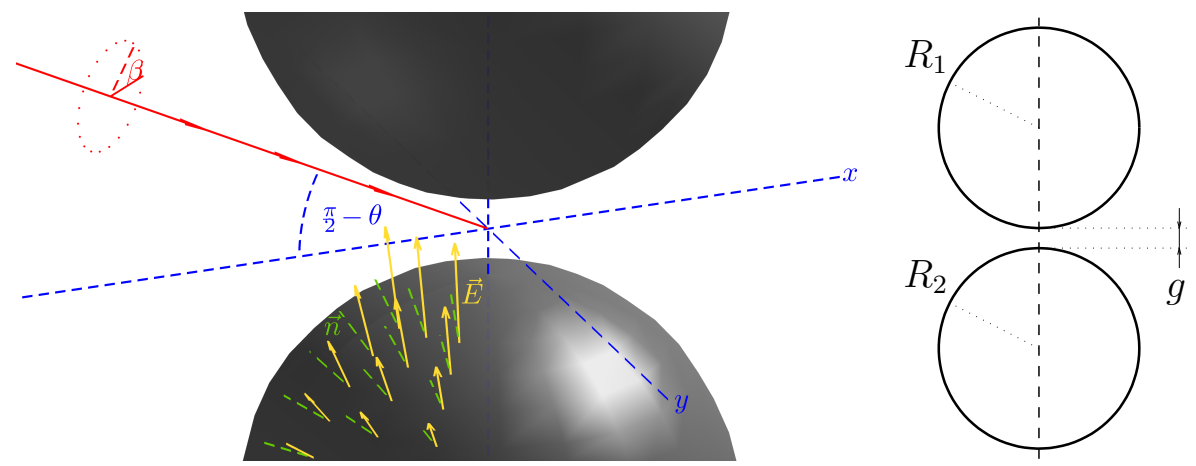

Figure 3.6 - Schematic highlighting the geometry of the dimer model (two spheres of radii $R_{1}$ and $R_{2}$, separated by a gap $g$ ). The symmetry axis of the dimer is the $z$-axis, with the origin in the center of the gap. The angle of incidence $\theta$ is defined as the angle between $\vec{k}$ (red line) and the z-axis; plane wave polarisation is fixed by $\beta$. Most importantly for SERS, is the study of the field ( $\vec{E}$, yellow vectors) on the surface of the sphere (with normals $\vec{n}$, green vectors).

of SERS: the em hotspot. At the resonance frequency of this geometry, the field re-distribution is extremely non-uniform; the incident field is 'funnelled' into a small region, resulting in a strongly localised, high em field intensity. As will become clear later, both, the field amplification and its localisation are crucial for the single molecule sensitivity observed in SERS.

It is convenient to introduce $F$ and $L$, which abbreviate the field enhancements in the $|\vec{E}|^{4}$-approximation as follows:

$$
F=\frac{\left|\vec{E}_{\mathrm{loc}}(\lambda)\right|^{4}}{\left|\vec{E}_{0}(\lambda)\right|^{4}}, \quad L=\log _{10} F
$$

$F$ is simply a new label for the EF in the $|\vec{E}|^{4}$-approximation (see Eq. 3.3), and $L$ is its decadic logarithm.

As stated before, this system can be solved analytically by GMT (see above), and a number of studies $[24,65,29,66]$ have explored this model and put it into the experimental SERS context. To lay the foundation for the 
discussion here, Fig. 3.6 summarises the model and its parameters. All calculations referred to in the following share the following details:

- sphere radii are identical at $R=R_{1}=R_{2}=25 \mathrm{~nm}$,

- spheres are separated by a gap of $g=2 \mathrm{~nm}$,

- sphere centers lie on the z-axis, at $R+g / 2$ and $-R-g / 2$,

- sphere material is Ag (dielectric function as above, see Eq. 3.18),

- outside medium is air, i.e. $\varepsilon_{M}=1$,

- excitation is a plane wave of linear polarisation,

- excitation $\vec{k}$ is fixed by a single angle $\theta=\angle\left(\vec{k}, \vec{e}_{z}\right)$, which is sufficient due to the symmetry of the problem.

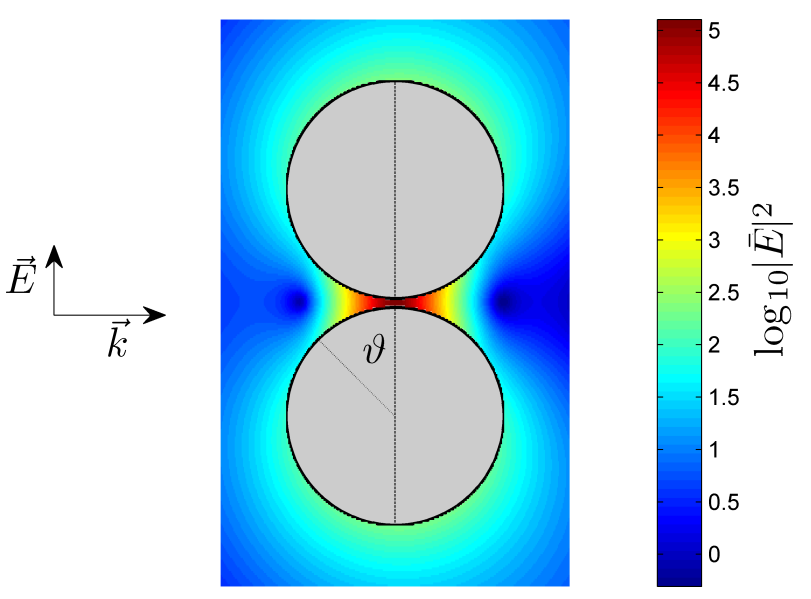

Figure 3.7 - Colour-map of $\log _{10}|\vec{E}|^{2}$ for the dimer of Ag spheres, as calculated by GMT. The dimer acts as a 'nano-antenna' and funnels field-lines into the gap between the spheres, resulting in a hotspot. In the $|\vec{E}|^{4}$-approximation, the hotspot shown here results in a $\sim 10^{10}$ enhancement factor. In this calculation, as indicated, $\vec{k}(\vec{E})$ is perpendicular (parallel) to the dimer axis, the outside medium is air (i.e. $\varepsilon_{\mathrm{M}}=1$ ), and plane wave excitation with $448 \mathrm{~nm}$ wavelength was used, which yields the maximum resonance in this configuration. 
Fig. 3.7 shows the near-field distribution in the vicinity of the dimer. As indicated in the figure, the incident wave propagates along $\vec{k} \| \vec{e}_{x}$ and is polarised along $\vec{E} \| \vec{e}_{z}$. This configuration yields the strongest maximum field amplification, which forms as the classical gap-hotspot. The field enhancement is not bound to the surface - it extends into space, albeit rapidly decaying with distance. This has repercussions for surface-enhanced fluorescence $(\mathrm{SEF})^{7}$, however, in the typical SERS case one assumes the analyte to adsorb onto the surface, and I will therefore, in the following, devote closer attention to the field at the surface.

It is sufficient to study the surface field of one of the two spheres: without loss of generality, I arbitrarily choose the sphere centered at $z=-R-\frac{g}{2}$; the field for the opposing sphere follows trivially from symmetry. Invoking spherical coordinates ${ }^{8}(R=$ const., $\vartheta, \varphi)$, the field on the surface can be written as a function thereof: $\vec{E}=f(\vartheta, \varphi)$.

Fig. 3.8 presents an overview of the near- and far-field properties of the dimer under different excitation geometries:

- (left column) $\vec{k} \| \vec{e}_{x}$ and $\vec{E} \| \vec{e}_{z}$, i.e. propagation $\vec{k}$ perpendicular, polarisation $\vec{E}$ parallel to the dimer axis,

- (center column) $\vec{k} \| \vec{e}_{x}$ and $\vec{E} \| \vec{e}_{y}$, i.e. both propagation and polarisation perpendicular to the dimer axis, and

- (right column) $\vec{k} \| \vec{e}_{z}$ and $\vec{E} \| \vec{e}_{x}$, i.e. propagation parallel, polarisation perpendicular to the dimer axis.

For these three cases, the following plots were generated:

\footnotetext{
${ }^{7}$ Fluorescence benefits from the field enhancement through enhanced absorption, but is also quenched since the metal adds non-radiative decay channels - in essence, due to the different distance dependencies of the two effects, the optimal position in SEF is not on, but above the surface. $[67,68]$

${ }^{8}$ In this work the physical convention is used, where $\varphi$ denotes the azimuthal angle $(0 \leq \varphi<2 \pi)$ and $\vartheta$ denotes the zenith $(0 \leq \vartheta \leq \pi)$.
} 

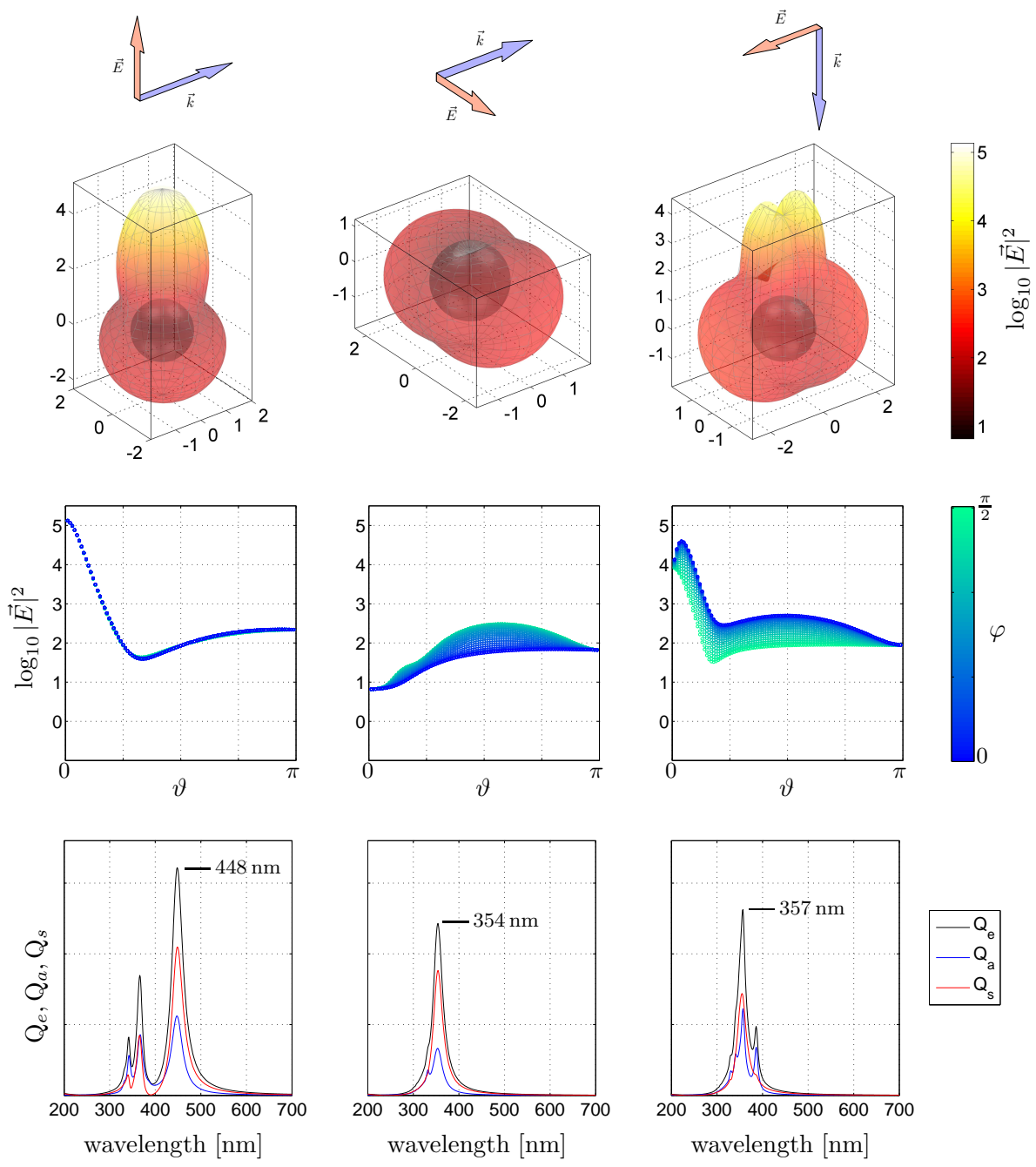

Figure 3.8 - Field distribution on the lower sphere in the dimer, for three different incident orientations (see top row). Each column in the plot array pertains to one excitation configuration and wavelength (to match the according resonance). The dimer (as described in Fig. 3.6) is excited with a polarised plane wave with $\vec{k}$ and $\vec{E}$ oriented as indicated, from left to right: $\left(\vec{k}\left\|\vec{e}_{x}, \vec{E}\right\| \vec{e}_{z}\right),\left(\vec{k}\left\|\vec{e}_{x}, \vec{E}\right\| \vec{e}_{y}\right)$ and $\left(\vec{k}\left\|\vec{e}_{z}, \vec{E}\right\| \vec{e}_{x}\right)$. The surface plots (top row) show the $\log _{10}|\vec{E}|^{2}$ distribution on the sphere by scaling the radius for each point (also note the colour-bar on the right) the undistorted unit-sphere (grey) is shown for comparison. For a more accessible portrayal of the same data (center row) $\log _{10}|\vec{E}|^{2}$ plotted vs $\vartheta$ and -by colour- vs $\varphi$ (see Fig. 3.6). (Bottom row) Extinction (black), absorption (blue) and scattering (red) coefficients vs wavelength. Note the strong field enhancements when $\vec{E} \| \vec{e}_{z}$ (left column), but also (and more surprisingly so) when $\vec{k} \| \vec{e}_{z}$ (right column). See text for details. 
- (top row) Plot of the local field by means of a distorted sphere surface - the local radius being varied by $r(\vartheta, \varphi) \propto \log _{10} \frac{\left|\vec{E}_{\text {|call }}\right|^{2}}{\left.||_{0}\right|^{2}}$. Note the logarithmic scaling - the field intensity is boosted by five orders of magnitude! In terms of enhancement, this accounts for the part which drives the (Raman- or fluorescent) dipoles on the surface. Squaring this expression (logarithm aside) yields $F$ (i.e. the $|\vec{E}|^{4}$-approximation, see above), which, in this case, predicts a Raman enhancement of roughly a factor of $10^{10}$ in the best case (left column; configuration $\vec{k} \| \vec{e}_{x}$ and $\vec{E} \| \vec{e}_{z}$, at $\vartheta=0$ ).

- (middle row) Essentially the same data as the top row, but displayed in a more conventional fashion (albeit less visual) - $L$ is plotted vs. the sphere's coordinates $\vartheta$ (on the abscissa) and vs. $\varphi$ (via colourbar). Note that for $\vec{E} \| \vec{e}_{z}$ the resulting local field has very little dependence on $\varphi$, whereas for $\vec{E} \perp \vec{e}_{z}$ the (now strongly) broken symmetry results in a strong $\varphi$ dependence. For example, consider the plot for $\vec{E} \| \vec{e}_{x}$ (right column): for $\vartheta=0$ and $\vartheta=\pi$ the enhancement is the same for all $\varphi$, but for $\vartheta=\pi / 2$ the enhancement ranges from $\log _{10}|\vec{E}|^{2}=2.7$ (at $\varphi=0)$ to $\log _{10}|\vec{E}|^{2}=1.9$ (at $\varphi=\pi / 2$ ). A similar dependence is observed for the case $\vec{E} \| \vec{e}_{y}$ (center column).

- (bottom row) Far-field properties: extinction (black), absorption (blue) and scattering spectrum (red). The axes limits are the same in all three plots. For $\vec{E} \| \vec{e}_{z}$, the resonance of the dimer is at $448 \mathrm{~nm}$, and thus in the visible region - as opposed to the $\vec{E} \perp \vec{e}_{z}$-situations, for which the resonance lies in the near UV. Due to the strength and spectral position of this resonance, the $\left(\vec{E} \| \vec{e}_{z}\right)$-configuration is the most useful for Raman experiments using excitation wavelengths in the visible range. Note that the resonances produced from the configurations studied here do not significantly shift under further variations of the incident geometry (i.e. $\theta$ and $\beta$, see Fig. 3.6). Instead, they simply de- or increase in strength, but stick to their spectral position. Hence, for any configuration $(\theta, \beta)$, the spectrum is well approximated by a superposition of the cases shown here. 
The configuration with $\vec{E} \| \vec{e}_{z}$ (left column in Fig. 3.8) yields a pronounced hotspot - in the gap, at the pole of the sphere (i.e. point $(0,0,-g / 2)$, or $\vartheta=0$ ), the field intensity is amplified by more than five orders of magnitude. Departure from this point yields a sharp drop in enhancement (note the $\log$-scale), at $\vartheta \sim \pi / 6$ the field intensity has dropped already by a factor of 100 with respect to the polar maximum.

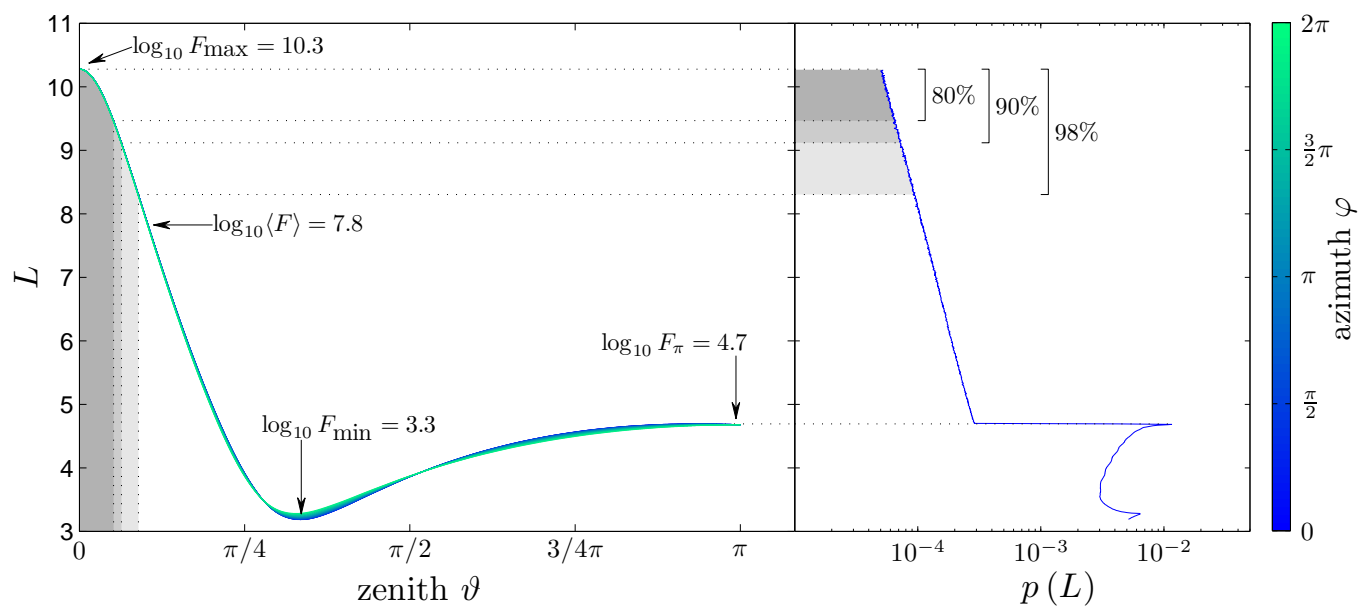

Figure 3.9 - (Left) Spatial enhancement distribution, $L$ in plotted vs. $\vartheta$ (and $\varphi$ as colours). Note that the maximum enhancement $F_{\max }$ corresponds to about $300\langle F\rangle$, i.e., in the $|\vec{E}|^{4}$-approximation, a single molecule experiencing the highest enhancement will yield (statistically speaking) as much intensity as $\sim 300$ randomly distributed molecules. (Right) probability density of the enhancement, $L$ is plotted vs. its probability. Note the logarithmic abscissa. A linear dependence of $\log p(L)$ emerges for $L>5$. In both plots, regions which, statistically, contribute $80 \%$ (dark gray), $90 \%$ (gray) and $98 \%$ (light gray) to the intensity are shown. See text for details.

Fig. 3.9 elaborates on exactly this point - a plot of $L$ (the decadic logarithm of the enhancement in the $|\vec{E}|^{4}$-approximation) vs. $\vartheta$ (left plot) is shown. Most strikingly, a molecule experiencing the maximum enhancement $\left(\log _{10} F_{\max }=10.3\right)$, i.e. located at the very hotspot center, contributes as much to the Raman signal as $\sim 300$ molecules on the average enhancement $\left(\log _{10}\langle F\rangle=7.8\right)$. In the statistical sense, this is equivalent to 300 
molecules randomly distributed on the sphere's surface ${ }^{9}$. Note that $L$ depends very little on $\varphi$, discernible only in the low enhancement region. Higher enhancements $(L>6)$ depend on $\vartheta$ only. From $L=f(\vartheta, \varphi)$ the probability distribution $p(L)$ (right plot) is extracted. Note the logarithmic scale on the abscissa, this is effectively a log-log plot of $p\left(|\vec{E}|^{4}\right) . \quad p(L)$ is the outcome of a simple Monte-Carlo simulation: with the local distribution of $F$ known on the entire sphere surface, one can evaluate the field in random spots ${ }^{10}$, thereby simulating a (large) series of SERS events, in each of which a single molecule probes the surface field. The histogram of enhancements, in the limit of an infinite number of simulated events ${ }^{11}$, equals the probability distribution $p(L)$.

If one were to randomly place a molecule anywhere on the sphere, collect its signal ('measuring' $L$ ) and do so over and over again, then $80 \%$ of the signal would come from only $0.66 \%$ of the sphere's surface (dark grey area; $0 \leq \vartheta<9.3 \mathrm{deg}$ ), despite the fact that these enhancements are the least likely to occur (see corresponding dark gray area in the plot on the right). $90 \%$ of the signal occurs from a $1 \%$ surface fraction (gray area, $0 \leq \vartheta<11.5 \mathrm{deg}$ ), while $98 \%$ stems from $2 \%$ of the surface (light gray area, $0 \leq \vartheta<16.1 \mathrm{deg})$. Conversely, for this latter case, the remaining $98 \%$ of the surface area contribute only $2 \%$ to the signal. This highlights the extremely skewed character of this long-tail distribution of enhancements, which is adequately described by a variant of the Pareto distribution (see below).

In Fig. 3.10 the polarisation is changed to $\vec{E} \| \vec{e}_{y}$ (corresponding to the center column in Fig. 3.8). The polarisation sensitivity of the dimer becomes evident here. In clear contrast to the earlier case, the field enhancement

\footnotetext{
${ }^{9}$ Let $n$ denote an ensemble of 300 random points on the sphere. Then, it holds that $300\langle F\rangle=\left\langle\sum_{n}(F)\right\rangle_{n}$, where the latter average is to be taken over all possible sets $n$. This may appear trivial, but precisely highlights the skewed distribution of $F$, since the equality only holds for the overall average; single sets $n$ will produce great variation.

${ }^{10}$ Random positions on a sphere, with a uniform surface density, are generated by $\varphi=2 \pi U$ and $\vartheta=\operatorname{acos}(2 U-1)$, where $U$ are uniformly distributed random numbers with $0 \leq U<1$.

${ }^{11}$ The number of simulated events was $10^{8}$; the histogram has 1000 bins.
} 


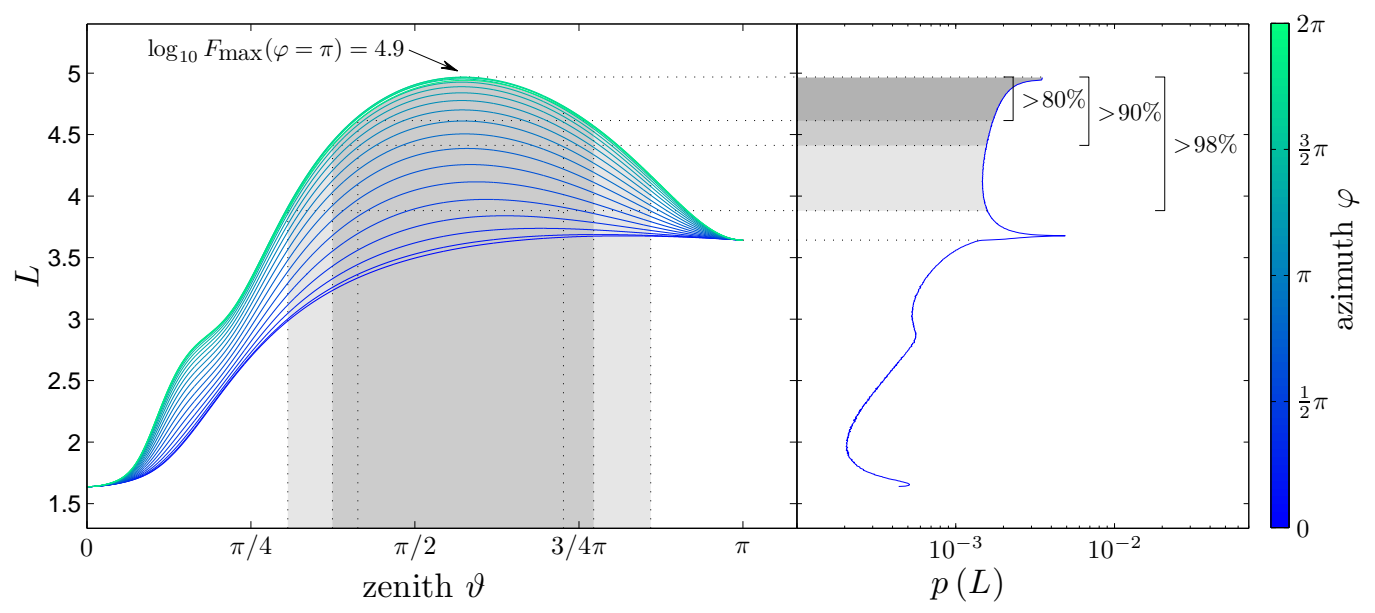

Figure 3.10 - (Left) Spatial enhancement distribution, $L$ in plotted vs. $\vartheta$ (and $\varphi$ as colours). Note the strong $\varphi$ dependence and the overall low enhancements for this case. (Right) probability density of the enhancement, $L$ is plotted vs. its probability. Note the logarithmic abscissa. In both plots, regions which, statistically, contribute at least $80 \%$ (dark gray), $90 \%$ (gray) and $98 \%$ (light gray) to the intensity are shown. See text for details.

here is comparably low $\left(L_{\max }<5\right)$ and spread out. Most of the signal stems from a large fraction of the surface area; the enhancement, when compared to the previously studied hotspot, is now delocalised. This is also evident in the probability distribution, since higher enhancements are more probable than low Ls. Note that, in this figure, the gray-ed areas do not directly correspond to the given signal intensity percentages. Due to the strong dependence in $\varphi$, one can no longer assert a single range for $\vartheta$, the correct surface boundary is now parametrised by both $\vartheta$ and $\varphi$. The corresponding ranges for $L$ now act as a lower bounds; they include at least $80 \%$ (dark gray), $90 \%$ (gray) and $98 \%$ (light grey) of the overall signal. Basically, this shows that the $\varphi$ dependence adds to the localisation - albeit not significantly so, in this case.

The third case, with $\vec{k} \| \vec{e}_{z}$ and $\vec{E} \| \vec{e}_{x}$, beats intuition and is therefore a bit of a surprise: although the dimer presents its minimal geometrical cross- 


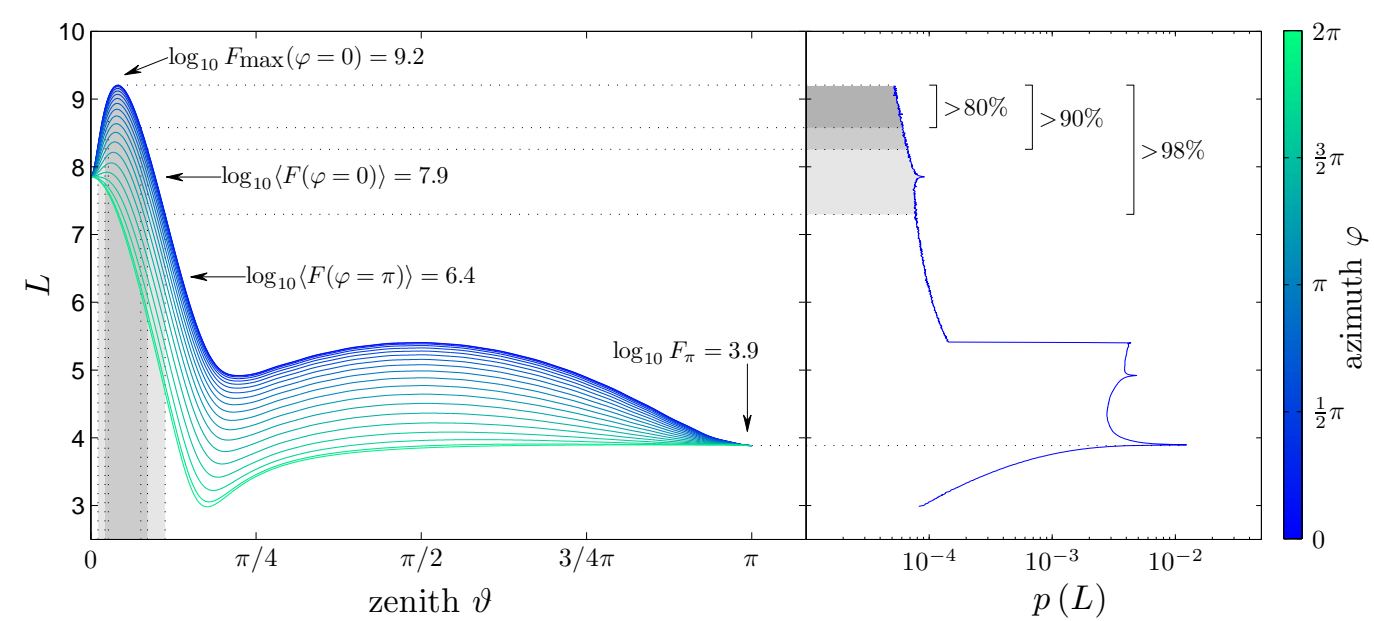

Figure 3.11 - (Left) Spatial enhancement distribution, $L$ in plotted vs. $\vartheta$ (and $\varphi$ as colours). Note the strong $\varphi$ for this case. (Right) probability density of the enhancement, $L$ is plotted vs. its probability. Note the logarithmic abscissa. In both plots, regions which, statistically, contribute at least $80 \%$ (dark gray), $90 \%$ (gray) and $98 \%$ (light gray) to the intensity are shown. See text for details.

section to the incident wave, the 'nano-antenna' nonetheless produces a strongly localised field redistribution, somewhat rivalling the hotspot studied in the first case (see Fig. 3.9). Two hotspots occur near (but not on) the pole, at $\vartheta= \pm 7.5 \mathrm{deg}$. The maximum enhancement is roughly one order of magnitude lower $\left(L_{\max }=9.2\right)$ than in the first case, however, it is strongly localised - as in the case of $\vec{E} \| \vec{e}_{y}$, the strong $\varphi$ dependence adds to the (already quite narrow) localisation in $\vartheta$. The probability distribution of enhancements is, again, a long-tail distribution.

Note that, in the cases where hotspots are apparent, the local electric fields therein are markedly normal to the surface. Therefore, analytes experiencing the enhancement of a hotspot will, if their orientation on the surface is fixed (or at least restricted), be exposed to a prevalent field direction, hence leading to the selective amplification of Raman dipoles, i.e. surface selection rules $[69,70,71]$. The orientation of the local field can not be chosen by the experimenter; it is a direct consequence of the $\mathrm{em}$ resonance 
in the dimer. For example, polarisation ratio measurements of liquid SERS samples [72] unanimously yield a ratio of $1 / 3$ for all modes and all analytes, thus exposing the dominant uniaxial response of hotspots. Accordingly, the local field provides a comprehensible explanation for the differences between normal Raman spectra and SERS spectra: the former measures -in general- orientationally averaged spectra, the latter exposes surface selection rules.

\section{Pareto distribution of enhancements}

In the cases discussed above, while safely ignoring all situations where $L<6$, it is visually obvious that $\log _{10} p(L)$ (note the log-scale in the plots) is (in a good first approximation) linear in $L$, monotonically decreasing and has an upper limit $L_{\max }$. We thus fit a straight line via:

$$
\log _{10} p(L)=-k L+c
$$

with parameters $k$ and $c$. Mathematically, this is equivalent to a Pareto distribution ${ }^{12}$ :

$$
p(F) \approx A F^{-(1+k)}
$$

The distribution is truncated by the maximum enhancement, so that $p(F)=$ 0 for all $F>F_{\max }$. Fitting the parameters for the case of $\vec{E} \| \vec{e}_{z}$ (see Fig. 3.9) yields $A=0.075, k=0.135$ and $\vec{F}_{\max }=1.9 \cdot 10^{10}$. The values of these parameters vary with the parameters of the model (i.e. sphere radii, etc...), however, once established, this analytical expression for the enhancement distribution proves useful in the simulation of large amounts of SERS events (i.e. for Monte-Carlo simulations). For applications, see Sec. 5.1.2, in particular Fig. 5.3, and Ref. [75].

\footnotetext{
${ }^{12}$ In his 1897 book Pareto [73] observed that $80 \%$ of the land in Italy is owned by $20 \%$ of the people. This skewed type of distribution is often encountered in economics (income distribution, sales per client, etc...), but also in other disciplines (e.g. articles per author, sand grain sizes, city sizes, file sizes, etc...); see Ref. [74].
} 


\section{Conclusions}

What emerges from these plots, and what carries beyond the limitations and approximations of this model, is the dominance of the hotspot in the local field enhancement. Regions of high enhancement are spatially very confined, for example as shown in Fig. 3.8, where $|\vec{E}|^{2}>4$ shows only when $0 \leq \vartheta<\pi / 8$, which corresponds to less than $4 \%$ of the surface area. The enhancement distribution outside the hotspot quickly becomes insignificant. The experimentally meaningful enhancement distribution is a long-tail, Pareto-like distribution in which the by-far biggest part of the signal stems from a very small fraction of the surface. Hence, even if a sphere (i.e. colloid) has a dense surface population of adsorbed analytes, only a very small fraction thereof will contribute to the observed signal. This is the foundation of the single-molecule sensitivity, to which I shall come back in chapter 5 .

The epigraph given at the start of this chapter quotes Fairthorne ${ }^{13}$ : "[...] a straight line law connecting any empirical data always can be achieved with the aid of suitably scaled logarithmic paper and a robust conscience." He adds: "Even more can be achieved if you give yourself the option of declaring the limits of the straight line portion only after you have plotted the data."

The astute reader will have noticed the similarity regarding the deduction of the truncated Pareto distribution of enhancements ${ }^{14}$.

\footnotetext{
${ }^{13}$ A highly commendable read, see Ref. [74]

${ }^{14}$ To console those who feel cheated, this does not mean that these findings are irrelevant or inaccurate. The empirical character in the deduction of the Pareto distribution of enhancements solely arises through Monte-Carlo simulations, which are based on known distributions (field distribution on the spheres, uniform random distribution of molecules thereon).
} 


\subsection{Chemical enhancement - controversy}

A much discussed issue in SERS EFs is the contribution from the so-called chemical enhancement. Its significance to the surface enhancement, in particular when invoked to explain enhancements orders of magnitudes above the theoretical limit given by em calculations, is -by and large- a recently refuted myth. The origin of this concept is three-fold, and I shall briefly digress into each case below.

\section{Early SERS}

Initially, in the early history of SERS [76], the term 'chemical enhancement' arose from the observation that a few simple molecules (e.g. pyridine, piperidine) experience a much higher enhancement (factor of 100-1000) than is theoretically predicted. This was credibly attributed to the chargetransfer (CT) mechanism [76, 77, 78]. Since CT is a concept mostly known in chemistry, and truly poses an enhancement for the specific molecules and substrates investigated, the term chemical enhancement became a concept in SERS, mostly used as a general umbrella denoting the more subtle aspects of surface chemistry, but associated with the rather misleading connotation of providing a significant enhancement in the general case.

A summary of the different effects proposed in favour of a chemical enhancement is found in section IV of Moskovits' 1985 review on surfaceenhanced spectroscopy [25]. Moskovits concludes:

"... the magnitude of this effect rarely exceeds a factor of 10 and is best thought to arise from the modification of the Raman polarisability tensor of the adsorbate resulting from the formation of a complex between the adsorbate and the metal. Rather than an enhancement mechanism, the chemical effect is more logically to be regarded as a change in the nature and identity of the adsorbate." 
Moskovits' notion of a modification of the Raman polarisability tensor due to the surface proximity is a much more sober formulation of the concept, since it the applies a more fitting taxonomy: modification, as opposed to enhancement.

\section{Single-molecule SERS}

Taking a step forward in history, the revival SERS experienced in the late 1990 's also lead to a revival of the chemical enhancement concept, but for the wrong reasons. Fueled by the apparent discrepancy between the muchquoted $10^{14}$ enhancement [79, 80, 81] (see discussion in Sec. 3.1.1) and the theoretically obtained $F_{\max } \approx 10^{11}$, it seemed necessary to invoke additional mechanisms to bridge this virtual gap in EFs. However, the dissonance in EFs is resolved by properly defining and measuring them [31,21]. Invoking Occam's razor [82], the idea of large chemical enhancements generally present in SERS is made largely redundant. As stated by Moskovits, and shown experimentally for a range of typical SERS analytes ${ }^{15}$ in Ref. [21], the mode-dependent modifications in the AEF (see Sec. 3.1.1) do not exceed a factor of 10. In fact, since these analytes are all known to adsorb well onto the used substrate (Lee \& Meisel Ag colloids, see chapter 4), it is not surprising that the variation in AEF barely exceeds a factor of 10 across analytes. Keep in mind that this does not rule out the existence of chemical effects on the enhancement; it merely provides a lower bound. However, since these results were obtained from a rather arbitrary set of analytes, it is equally important to recognise that one cannot claim large chemical enhancements without providing the according experimental verification.

\section{Salt activation in SERS}

Despite the overwhelming evidence that identifies the em effect as the major enhancement contributor, the discussion about the role of a chemical

\footnotetext{
${ }^{15}$ Benzotriazole and the dyes BTZ2, Rh6G and crystal violet
} 
enhancement has still not settled fully (for recent examples, see Otto et al. [83] and Schneider et al. [84]). One of the reasons for the ongoing belief in a chemical enhancement is the fact that from the first experiments on silver electrodes, to the commonly-used silver colloids nowadays, it was always necessary to use a salt solution to achieve an 'active' substrate/suspension. Potassium chloride was used as a reduction agent in the oxidization/reduction-cycles for the roughening of the silver electrodes, and is used to activate Ag colloid suspensions too. For colloidal solutions however, salts are known to induce aggregation ${ }^{16}$, which exposes the boost in EF as an em effect (the dimer of spheres yields a much higher maximal field enhancement than the single sphere [17]).

Yet, until very recently one particular observation stood in opposition to this conclusion. From many different salts studied, $\mathrm{NaCl}$ and $\mathrm{KCl}$ clearly show the highest enhancements when applied to the commonly used citrate reduced Lee and Meisel Ag colloids [23], showing a up to $\sim 20$-fold signal increase (dependent on the probe molecule) when compared to other salts like $\mathrm{KNO}_{3}, \mathrm{KPF}_{6}$. Since the valence of the dissociation products of these salts is the same and thus the same screening and aggregation is expected, this observation lends some credibility to the proposition that a more subtle (i.e. 'chemical') effect might be underway. However, it has been shown recently $[87,88]$ that halides $\left(\right.$ e.g. $\mathrm{Cl}^{-}$) replace the citrate layer on the colloid surface, thereby potentially changing surface charge, colloid stability, adsorption and interaction properties. This explains the variance in enhancements across aggregation agents.

In conclusion, chemical enhancement is an inaccurate, but historically coined term which may refer to a number of interesting effects (e.g. the CT

\footnotetext{
${ }^{16}$ The dissociated salt ions act as electrolyte that effectively screens away the charge of the like-charged colloids, thus reducing repulsion (see DLVO theory, section 4.2). This behaviour obviously depends on the salt concentration, and a standard procedure [85] (also given in [86], p. 94) has been proposed to obtain the critical coagulation concentration at which no repulsion occurs between the colloids. See chapter 4 for details.
} 
mechanism is a thriving topic in SERS ${ }^{17}$ ). Unfortunately, it is often used as a carpet under which apparent discrepancies in enhancements are swept.

\footnotetext{
${ }^{17}$ A good precis of the CT mechanism can be found in Ref. [89], see Tian's comment on page 156.
} 


\section{Chapter 4}

\section{Self-limiting colloidal aggregation in SERS-active solutions}

The ships hung in the sky in much the same way that bricks don't.

Douglas Adams

\subsection{Noble metal colloids in SERS}

This chapter focusses on the physics of silver colloids suspended in water, which are commonly used as SERS substrates ${ }^{1}$. In fact, all experimental work discussed in this thesis is footed upon this basic system. The chapter aims to examine the dynamics of one of the most important effects of colloidal sols for SERS: colloidal aggregation. Particular emphasis will be

\footnotetext{
${ }^{1}$ The term 'substrate', as it is used here and in the remainder of this thesis, encompasses any surface to which the analyte molecules may attach and is therefore not restricted to the intuitive understanding as a solid-to-gas interface. For example, metal colloids in water, i.e. a solid-to-liquid interface, constitute a SERS substrate.
} 
given to the important, yet somewhat counter-intuitive effect of self-limiting colloidal aggregation.

The chapter is divided into two parts. The first part (Secs. 4.1-4.3) will characterise the experimental system and lay the foundation for the subsequent discussion. In detail, I first introduce the nature and basic properties of a typical colloidal sol for SERS (this section), followed by a brief treatise of $\mathrm{DLVO}^{2}$ theory, which is an approximation to the pair-wise interaction of colloids, in Sec. 4.2. Electron micrographs of single and aggregated particles are shown in Sec. 4.3. The second part of the chapter (Secs. 4.44.5) applies this knowledge: Sec. 4.4 presents in-situ SERS experiments which yield insights into the colloidal aggregation, and Sec. 4.5 provides, by means of kinetic Monte-Carlo simulations, a qualitative explanation of the self-limiting behaviour in the aggregational dynamics.

\subsubsection{Introduction}

Colloids are many-atom particles ranging in size from a few nanometers to microns ${ }^{3}$, which are suspended in a medium. Both colloids and medium may be gaseous ${ }^{4}$, liquid or solid ${ }^{5}$. The existence of colloidal suspensions depends on a fine balance of forces: hard-core repulsion, Coulomb attraction and repulsion, van der Waals attraction, adhesive and cohesive intermolecular forces, and hydrodynamic forces (coupling through movement). For stable colloids, these forces necessarily must be in equilibrium - which is found to be the case for a great variety of conditions, leading to a manifold of colloidal systems. Naturally occurring forms of colloidal sols are, for example, fog, milk, opal and bone; examples of synthetic colloids are aerogel, paint and stained glass. Colloids are mostly studied in the

\footnotetext{
${ }^{2}$ Named after its developers: Derjaguin, Landau, Verwey and Overbeek.

${ }^{3}$ Due to their size and charge colloids are sometimes referred to as macroions, see for example Refs. [90, 91].

${ }^{4}$ Colloids suspended in a gas are referred to as aerosols.

${ }^{5}$ Note that colloidal suspensions exist for all combinations of aggregate states for colloids and medium, except where both are gaseous.
} 
context of surface chemistry (since the large surface areas involved provide a very efficient pathway for catalysis); detailed investigations can be found in the specialised literature $[92,86,93]$.

In SERS, colloidal sols of metal nano-particles suspended in a liquid (usually water) are a commonly used substrate. The move to use colloids as a SERS substrate stems from: (i) the fact that they were readily available (see next section) and (ii) from the understanding of the surface-enhancement as an em effect, relying on roughened surfaces:

- SERS was first observed on electrochemically roughened silver electrodes [4], and the enhancement was soon attributed to the electric field enhancement stemming from the surface roughness [5]. Colloids, equivalently, provide the surface 'roughness' simply through their surface curvature.

- In the single-colloid regime a significant surface enhancement (field enhancement $\left.\frac{\left|\vec{E}_{\text {ocal }}\right|}{\left|\vec{E}_{0}\right|} \sim 10, \rightarrow F \approx 10^{4}\right)$ is already present, but, as discussed in Sec. 3.1.5, small aggregates function as "nano-antennas" and can provide a dramatically increased field enhancement $(F \approx$ $10^{10}$ ) in the vicinity of the hotspot.

- Moreover, depositing colloids onto a base substrate (e.g. glass, silicon) is a simple method ${ }^{6}$ to create dry, roughened noble metal surfaces $[9,94,95,96]$ (see Sec. 4.3).

Hence, the state of aggregation in a colloidal sol plays a crucial role in SERS [97]. In this context, Table 4.1 draws an analogy between the state of aggregation in conventional matter and in a colloidal sol.

The 'phase transition' from single colloids to aggregated colloids, which is achieved by means of adding a salt (see Sec. 4.2), is referred to as SERSactivation. This process, and its subtleties, are the topic of this chapter.

\footnotetext{
${ }^{6}$ The simplicity is highlighted by Fig. 1 in Ref. [94]
} 


\begin{tabular}{ll}
\hline normal matter & colloidal sol \\
\hline gaseous & $\begin{array}{l}\text { single colloids: the suspension of single col- } \\
\text { loidal particles in a liquid solution; interact- } \\
\text { ing only through elastic collisions (via Coulomb } \\
\text { repulsion) } \\
\text { flocculated colloids: colloidal particles are associ- } \\
\text { ated by a weak short-range force; reversible } \\
\text { coagulated colloids: colloidal particles are } \\
\text { bound strongly through short range interaction; } \\
\text { irreversible }\end{array}$ \\
\hline
\end{tabular}

Table 4.1 - Analogy between phases of aggregation in normal matter and in colloidal sols.

As described previously (chapter 3), SERS relies on surface plasmons (resonances in the visible range with little damping) supported by noble metals - i.e. silver and gold. Ag and Au colloids became readily available when Lee \& Meisel, in 1982, published a range of simple recipes for the synthesis of these colloids [23]. Since all SERS experiments carried out in the course of this work used Lee \& Meisel Ag colloids as a substrate, I shall, in what follows, provide a closer look at this system.

\subsubsection{Lee \& Meisel silver colloids}

\section{Synthesis}

The Lee \& Meisel approach [23] is a 'wet' chemistry, 'bottom-top' approach: for Ag colloids, an aqueous solution of metallic ions is prepared $\left(\mathrm{AgNO}_{3}\right)$, which, when the solution is heated, nucleate into small clusters of atoms which grow until the solution is depleted of silver ions. With the cooling of the solution an organic capping layer (sodium citrate) forms on the colloid's 
surface and prevents further growth. This capping layer dissociates in aqueous solution, creating a net-charge on each colloid, and turning the suspending water into an electrolyte. The correspondingly simple recipe ${ }^{7}$ produces colloids well-suited for SERS (roughly spherical shapes with a mean diameter of $\sim 50 \mathrm{~nm}$ ). Thus understandably, Lee \& Meisel Ag colloids have found wide adoption by the SERS community.

The final colloidal sol is said to be charge-stabilised: the like charge present on each colloid causes Coulomb forces repelling colloids from one another, hence inhibiting aggregation and the consecutive precipitation.

\section{$\mathrm{UV} /$ Vis characterisation - extinction}

The Ag colloidal sol is a dense, green-brown tinted liquid. The extinction spectrum, measured using a double beam spectrometer, is shown in Fig. 4.1 (black line). Prior to measurement the sol was diluted by half (with distilled water) to minimise multiple scattering. Although this changes the electrolytic properties of the supernatant, it does not appear to have an effect on the aggregational state of the particles. Theoretically derived extinction coefficients (obtained from Mie theory calculations; normalised) of a single Ag spheres with radius $a=25 \mathrm{~nm}$ (red line), $a=30 \mathrm{~nm}$ (green line) and $a=35 \mathrm{~nm}$ (blue line) are shown for comparison. The measured extinction peaks at $\sim 430 \mathrm{~nm}$, which is roughly consistent with the calculated plasmon resonances (the extinction peaks at 417,428 and $442 \mathrm{~nm}$, respectively), thus supporting the approximate figure for a mean colloid diameter of $\sim 50-60 \mathrm{~nm}$. Note that although the calculation for a sphere of $30 \mathrm{~nm}$ radius corresponds best to the observed extinction spectrum, this is not necessarily the most probable particle size to be found in the solution. The observed extinction is a weighted superposition of the spectra of all absorbing and scattering components of the sol - including spheres

\footnotetext{
${ }^{7}$ The recipe is short enough to be reproduced here, quote: " $A \mathrm{gNO}_{3},(90 \mathrm{mg})$ was dissolved in $500 \mathrm{~mL}$ of $\mathrm{H}_{2} \mathrm{O}$ and brought to boiling. A solution of $1 \%$ sodium citrate $(10 \mathrm{~mL})$ was added. The solution was kept on boiling for ca. $1 \mathrm{~h} . "$
} 
and other shapes of various physical dimensions (which are observed in electron microscopy images, see Sec. 4.3).

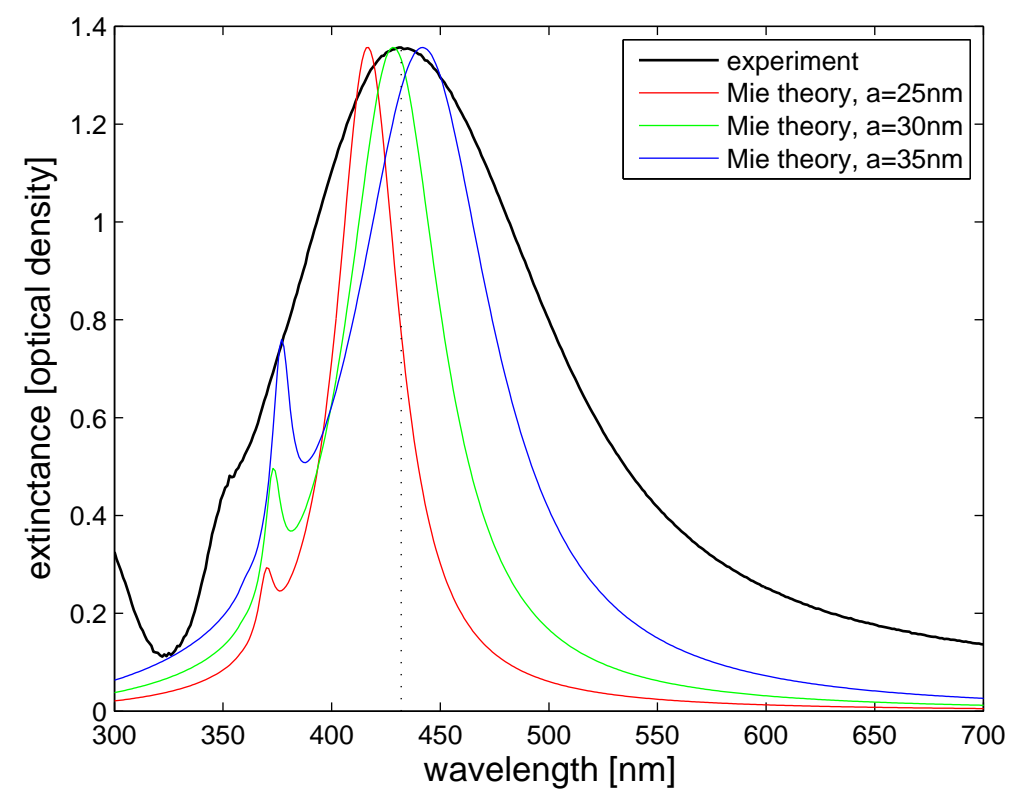

Figure 4.1 - Extinction spectrum of Lee \& Meisel Ag colloids. To minimise multiple scattering the sol was diluted by half using distilled water. The experimental spectrum (black solid line) peaks at $430 \mathrm{~nm}$ (indicated by dotted line). Three theoretical spectra (obtained by Mie-theory) for different sphere radii $a$ are drawn, $a=25 \mathrm{~nm}$ (red, peaks at $417 \mathrm{~nm}$ ), $a=30 \mathrm{~nm}$ (green, peaks at $428 \mathrm{~nm}$ ) and $a=35 \mathrm{~nm}$ (blue, peaks at $442 \mathrm{~nm}$ ).

Assuming the average colloid to be a sphere with radius $a=30 \mathrm{~nm}$, it is trivial to calculate

- the volume of a colloid: $V=4 \pi / 3 a^{3}=1.13 \cdot 10^{-16} \mathrm{~cm}^{3}$ and

- the mass of a colloid: $m=\rho V=10.49 \mathrm{~g} / \mathrm{cm}^{3} \cdot V=1.19 \cdot 10^{-15} \mathrm{~g}$

By dividing the initial mass of silver ${ }^{8}$ with the mass of a colloid, one can estimate the number density of colloids in the sol:

$$
\rho_{\text {colloids }} \approx 5 \cdot 10^{11} \frac{\text { colloids }}{\mathrm{cm}^{-3}}
$$

\footnotetext{
${ }^{8}$ The initial $90 \mathrm{mg} \mathrm{AgNO}_{3}$ (per $100 \mathrm{ml}$ ) contain roughly $\sim 57 \mathrm{mg}$ silver.
} 
Despite its approximate nature, this is an important number which, together with the analyte concentration (and a robust conscience on its adsorption efficiency) gives an estimate of the average surface number density of analytes per colloid, $N_{\text {surf }}$. This is, for example, of value for the estimation of the SSEF (see Sec. 3.1.1, Eq. 3.5).

Note that the estimate of $\rho$ assumes all silver present in the solution to nucleate into colloids. As a necessary condition for this to happen, the amount of reducing agent (sodium citrate) in the Lee \& Meisel recipe must be sufficient. The additional criterion, which is hard to meet in practice, is that no other nucleation sites than growing colloids occur in the sol. In the reality of the chemistry lab, however, one often finds a mirror finish indicating a silver coating on the inside of the beaker. In conclusion, the colloidal number density $\rho$, as estimated above, therefore merely states the approximate upper limit.

\subsubsection{Some puzzling observations}

It is well known [83] that, in the SERS regime, Lee \& Meisel colloids need to be activated, by adding a salt (in our case $\sim 10 \mathrm{mM} \mathrm{KCl}$ ), to produce large enhancements. As is visible by eye, higher concentrations of $\mathrm{KCl}$ (i.e. $>40 \mathrm{mM}$ ) lead to rapid coagulation, leaving a clear solution with dark precipitate at the bottom after a relatively short time $(<1 \mathrm{~h})$. Thus, inducing aggregation seems to be the main effect of the $\mathrm{KCl}$ addition. This is consistent with the predictions of electromagnetic theory: clusters of colloids are capable of much higher enhancements than single colloids. However, the precise mechanism of this activation is somewhat disputed and interwoven with the long-standing discussion and controversy on the magnitude of the chemical enhancement (see Sec. 3.2).

Chemical enhancements caused by salt ions appear a far-fetched theory, and hence it suggests itself to assign the salt's effect to colloidal aggregation. This, however, yields a puzzle: Lee \& Meisel Ag colloids provide, upon 
addition of $10 \mathrm{mM} \mathrm{KCl}$, high enhancements are thus presumed to have aggregated, yet the colloids do not precipitate from the solution. Such sols appear to be stable for months - and still provide large enhancements expected only from aggregated colloids.

In summary, the following questions arise:

- What is the effect of $\mathrm{KCl}$ on colloids? How is aggregation induced?

- Can colloidal aggregation alone explain the experimentally observed increase in enhancement?

- Is colloidal aggregation reversible? Do SERS signals arise from stable clusters or are the involved geometries transient (i.e. flocculation as opposed to coagulation: dynamic pairing)?

- Why are colloidal sols with low salt concentrations stable, yet provide large enhancements? Once aggregation can take place, what prevents a full collapse of the sample?

These are basic questions pertaining directly to the physical and chemical mechanisms in SERS. Consequently, they will be addressed throughout the course of this chapter. As stated initially, what defines the existence and aggregational state of colloids in solution is a balance of forces; the interplay of repulsive and attractive forces amongst colloids, in an electrolytic solution, governs the dynamics of colloids in the sol. To put this notion on a theoretical foundation, the next section is devoted to model and approximate the overall force as a function of colloid size, charge, and -in particular- salt concentration.

\subsection{DLVO theory}

In the 1940s [98] developed a theory (commonly abbreviated as DLVO theory) that describes the electrostatic pair interaction of charged particles 
in an electrolyte solution. This theory is of particular importance since it describes the aggregation dynamics that are expected from a colloidal suspension upon salt addition. Approximations aside, this theory leads -in principle- to the pair interaction potential of two colloids, which could be used to put numbers to the rate constants of cluster formation as a function of the colloid concentration and diffusion constant.

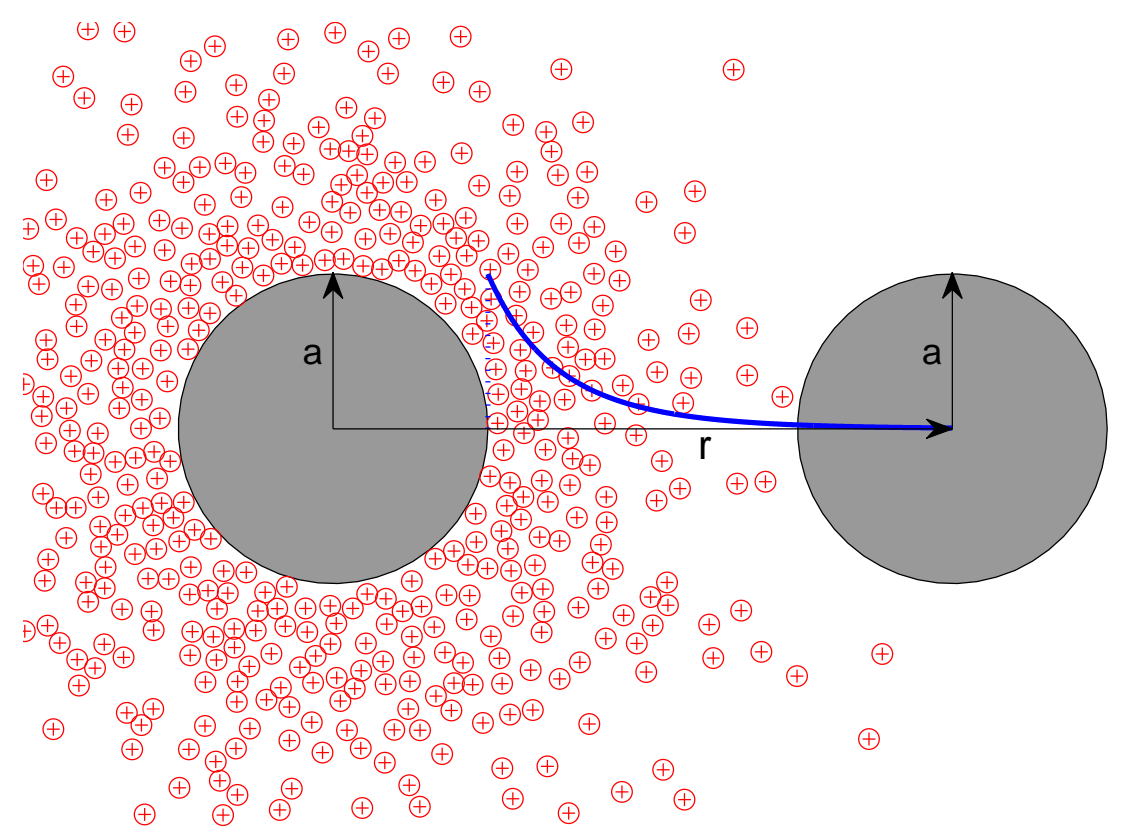

Figure 4.2 - Schematic depiction of the model system. Shown are two colloids of radius $a$ with center-to-center separation $r$. Since Ag colloids are negatively charged and reside in an electrolyte, positive ions in the solution will be drawn near the colloids and thus screen their charge. This is explicitly depicted for the colloid on the left. The resulting electrostatic part of the potential $U \propto \exp (-\kappa r) / r$ is also plotted (blue line) as a function of $r$. Note that the concentration of positive ions in regions close to the colloid may be limited due to the size of the ions, thus resulting in a well-ordered layer on the colloid's surface (Stern layer). This is not accounted for by DLVO theory. Also note that although identical colloids are assumed here, in general one is not limited to this case.

The DLVO interaction potential takes account of two fundamental forces present in this system: 
- screened Coulomb force: the like-charged colloids repel each other due to Coulomb's force, however, the ions present in the electrolytic solution screen the charges and thus modify the mutual repulsion of colloids.

- van der Waals force/Casimir force: this intermolecular force stems from electromagnetic fluctuations which provides the grounds for electrostatic interactions (i.e. dipole-dipole, etc...); it is referred to as van der Waals force in the case of interacting molecules and as Casimir force in the case of bulk materials. It constitutes an attractive force at short distances between the interacting entities. The rapid onset of attraction, followed by the hard-core repulsion as soon as the molecules/surfaces get too close, is often modeled using a LennardJones type potential.

\subsubsection{Screened Coulomb repulsion}

The initial problem is to analytically solve the Poisson-Boltzmann (PB) equation (Eq. 4.2), which governs the electric field configuration in the presence of freely movable charge carriers (e.g. ions), given some boundary conditions. The PB equation can be derived from the Law of Gauss:

$$
\nabla \cdot D=\rho,
$$

with $D=\varepsilon_{0} \varepsilon_{r} E$ and $\mathbf{E}(\mathbf{r})=-\nabla \Phi(\mathbf{r})$ follows:

$$
\Delta \Phi(\mathbf{r})=-\frac{e}{\varepsilon_{0} \varepsilon_{r}} \sum_{i=1}^{m} z_{i} \rho_{i} g_{i}(\mathbf{r})
$$

Here $e$ is the electron's charge, $i$ addresses the different types of electrolyte ions, $z_{i}$ is the corresponding ions valence and $\rho_{i}$ is the ion number density, $\rho_{i}=N_{i} / V$. The factor $g_{i}(\mathbf{r})$ accounts for the Boltzmann distribution of ions in the electrical potential:

$$
g_{i}(\mathbf{r})=\exp \left(-\beta z_{i} e \Phi(\mathbf{r})\right)
$$


Thus, the PB equation is an inhomogeneous, second order, nonlinear differential equation. The DLVO approach is to approximate the exponential term by expanding it into a power series which is truncated after the first order term:

$$
g_{i}(\mathbf{r})=1-\beta z_{i} e \Phi(\mathbf{r}) \quad .
$$

By substituting $g_{i}$ in Eq. 4.2 the PB equation is transformed into the so-called Debye-Hückel equation, which for the case of two spherical macroions can be solved analytically. The result is:

$$
\frac{U(r)}{k_{B} T}=\frac{e^{2} Z^{2}}{4 \pi \varepsilon k_{B} T}\left[\frac{e^{\kappa a}}{1+\kappa a}\right]^{2} \frac{e^{-\kappa r}}{r}+\frac{V(r)}{k_{B} T},
$$

where $r$ is the center-to-center separation of spheres of radius $a$ with charges $Z$. $\mathcal{K}^{-1}$ is the Debye-Hückel screening length of the electrolyte which can be calculated from the concentrations $n_{j}$ and charges $z_{j}$ of the involved ions:

$$
\kappa^{2}=\frac{e^{2}}{\varepsilon k_{B} T} \sum_{j=1}^{N} n_{j} z_{j}^{2} .
$$

All the other symbols have the standard meaning. The exponential decay of the potential is a result of the screening.

\subsection{2 van der Waals attraction}

The second component of the total effective potential is the van der Waals interaction $V(r)$. This interaction applies to molecules and bulk materials - the common cause are electromagnetic fluctuations creating transient dipoles, etc... The interaction can then be described in the framework of electrostatics, in which dipole-dipole interactions are the dominating term. The theoretical foundations for the intermolecular forces where laid out by London [99] and are extended to bulk materials by Hamaker theory [86]. The basic idea is as follows: the dominating interaction is of the type dipole-dipole, the potential being:

$$
V_{\text {dipole }}=-\frac{C}{r^{6}},
$$


where $R$ is the distance in between the interacting molecules and $C$ is a constant which depends on their respective wavefunctions. Principally, this is valid for every point within the volume of the interacting colloids thus, for an infinitesimally small volume $d \eta$ located within colloid $A$, the potential energy $d V_{A, \eta}$ is the integral:

$$
d V_{A, \eta}=-\int_{B} \frac{C_{\eta, v}}{r_{\eta, v}^{6}} d v,
$$

integrating over all infinitesimally small volumes $d v$ contained in colloid $B$. The overall dipole-dipole based interaction potential then is the integral of Eq. 4.8 over colloid $A$ :

$$
V_{A, B}=-\int_{A} \int_{B} \frac{C_{\eta, v}}{r_{\eta, v}^{6}} d v d \eta
$$

Evaluating Eq. 4.9 for the specific case of two spheres of radii $a$, separated by $r$ (center-to-center), yields [86]:

$$
V(r)=-\frac{A_{\text {eff }}}{6}\left[\frac{2 a^{2}}{r^{2}-4 a^{2}}+\frac{2 a^{2}}{r^{2}}+\ln \left(1-\frac{4 a^{2}}{r^{2}}\right)\right],
$$

where $A_{\text {eff }}$ is the Hamaker constant, which depends on the materials of the objects under consideration and on the medium in which they are immersed in [100]. This model of the van der Waals interaction potential diverges with $r \rightarrow 2 a$. However, upon contact, the solid colloids will experience a strong (hard-core) repulsion. This is accounted for by simply postulating the hard-core repulsion, i.e. $V(r \leq 2 a)=\infty$.

The sum of Eq. 4.5 and Eq. 4.10, plus the hard-core repulsion at contact, constitutes the DLVO interaction potential. The full expression for two colloids $A$ and $B$ of variable charge and radius is:

$$
V_{D L V O}=\left\{\begin{aligned}
r>2 a: & \frac{e^{2} Z_{A} Z_{B}}{4 \pi \varepsilon} \frac{e^{k a_{A}}}{1+\kappa a_{A}} \frac{e^{\kappa a a_{B}}}{1+\kappa a_{B}} \frac{e^{-\kappa r}}{r} \\
& -\frac{A_{\text {eff }}}{6}\left[\frac{2 a_{A} a_{B}}{r^{2}-\left(a_{A}+a_{B}\right)^{2}}+\frac{2 a_{A} a_{B}}{r^{2}-\left(a_{A}-a_{B}\right)^{2}}+\ln \left(\frac{r^{2}-\left(a_{A}+a_{B}\right)^{2}}{r^{2}-\left(a_{A}-a_{B}\right)^{2}}\right)\right] \\
r \leq 2 a: & \infty
\end{aligned}\right.
$$




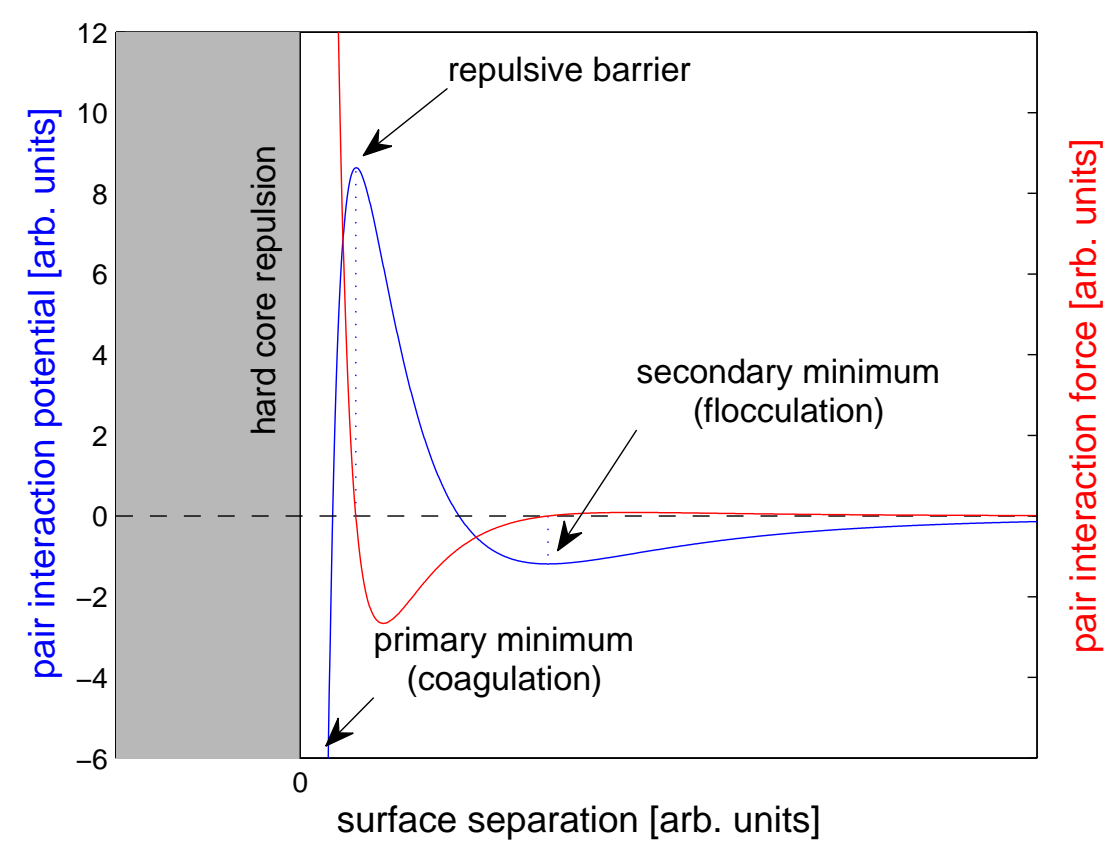

Figure 4.3 - Qualitative pair-potential of colloidal particles. Shown is the potential as a function of colloid surface-to-surface distance. Three 'phases' can be identified: (i) free phase, colloids interact through elastic collisions and remain single particles; (ii) flocculated phase, colloids bind reversibly without contact through a secondary minimum in the potential surface; (iii) aggregated phase, colloids touch $(r=2 a)$ and bind irreversibly.

In the case of two identical spheres this simplifies to:

$$
V_{D L V O}=\left\{\begin{array}{l}
r>2 a: \frac{e^{2} Z^{2}}{4 \pi \varepsilon}\left(\frac{e^{\kappa a}}{1+\kappa a}\right)^{2} \frac{e^{-\kappa r}}{r}-\frac{A_{e f f}}{6}\left[\frac{2 a^{2}}{r^{2}-4 a^{2}}+\frac{2 a^{2}}{r^{2}}+\ln \left(1-\frac{4 a^{2}}{r^{2}}\right)\right] \\
r \leq 2 a: \infty
\end{array}\right.
$$

Despite their approximate character, applying these relations to (idealised) Lee \& Meisel colloids provides a basic understanding, and holds qualitative conclusions, for the aggregational dynamics observed in SERS.

Fig. 4.3 shows the qualitative nature of the pair-wise interaction potential (blue line) as it is modeled by the DLVO formalism. The following contributions to the potential emerge: (i) the 'long-range' repulsive Coulomb potential which is caused by the colloidal charge, but partly screened by the 


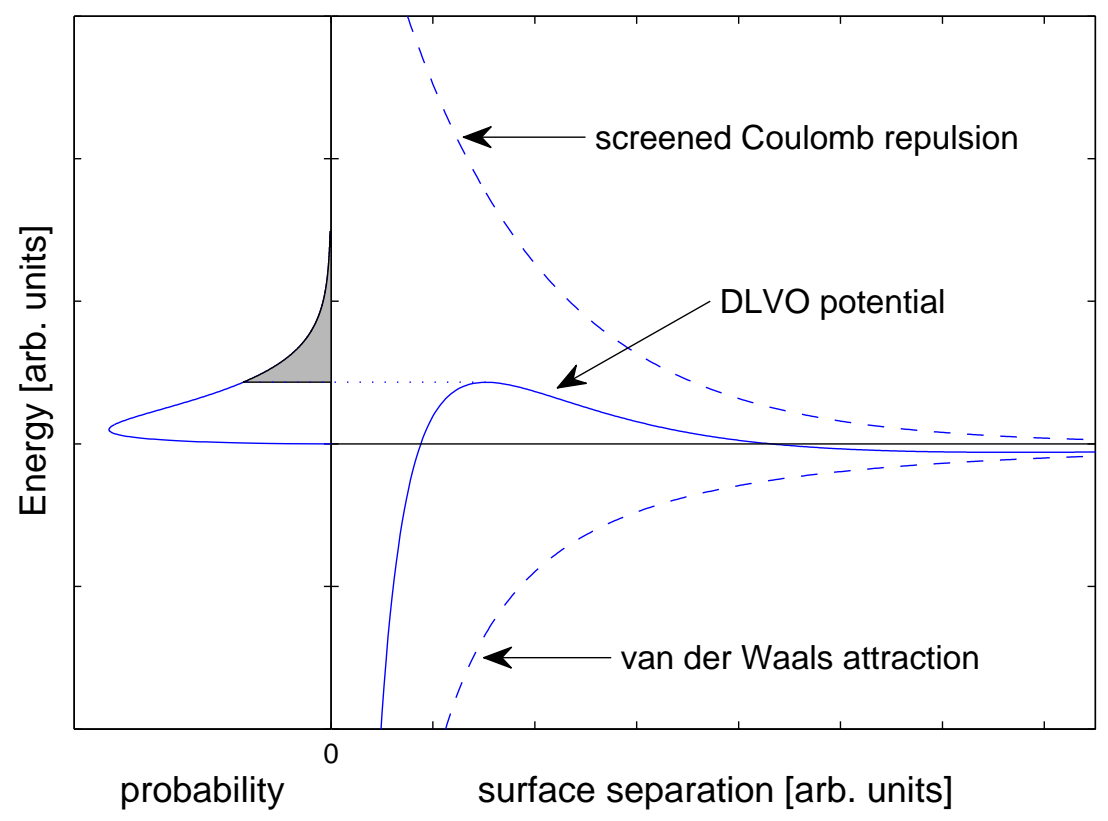

Figure 4.4 - Components of a qualitative DLVO potential leading to a repulsive barrier (right plot), and its relation to the Maxwell-Boltzmann distribution of kinetic energies (left plot).

ionic environment and (ii) the short-range van der Waals potential which creates the primary (coagulation) and shallower, secondary potential minimum (flocculation). The red line in Fig. 4.3 traces the force as a function of colloid distance (positive force meaning attraction, negative force repulsion). The parameters (colloid radius and charge, Hamaker constant) in this plot were chosen to showcase the qualitative nature of the DLVO potential, emphasising both primary and secondary potential minimum however, when choosing parameters to realistically model Lee \& Meisel Ag colloids, the secondary minimum vanishes (to a good approximation).

Thus, in this model of Ag colloids, the flocculation phase is absent, and the coagulation phase is shielded by a repulsive barrier from the single colloid phase. Picture a collision in which a resting colloid $A$ is approached by a colloid $B$ with kinetic energy $E_{B}$. This barrier defines the threshold for the energy which colloid $B$ needs to overcome repulsion, and hence to aggregate with colloid $A$. As colloids are assumed to undergo Brownian 
motion [101], their kinetic energies will be Maxwell-Boltzmann probability distributed [102]:

$$
f(E)=A \exp \left(\frac{-E}{k_{B} T}\right), \quad A=2 \sqrt{\frac{E}{\pi\left(k_{B} T\right)^{3}}} .
$$

The connection between the repulsive barrier and the probabilistic distribution of kinetic energies is depicted in Fig. 4.4. The mechanism underlying colloidal aggregation, and therefore the inherent thermodynamical instability of any colloidal sol, can now be explained: the fraction of colloids with kinetic energy sufficient to overcome the repulsive barrier will aggregate in a collision event; and the Boltzmann-distribution of kinetic energies will always yield a finite, non-zero probability for a colloid to have this kinetic energy or more. The height of the repulsive barrier with respect to the Boltzmann-distributed energies then defines how large that fraction will be. For example, assuming a barrier height of exactly $k_{B} T$, in $57 \%$ of all collision events colloids will stick. For a $2 k_{B} T$ barrier this reduces to $26 \%$, and for a $10 k_{B} T$ barrier it drops to $\sim 2 \%$. Therefore it makes sense to refer to a colloidal sol as metastable since aggregation/coagulation always occurs. However, depending on the interaction potential, this may happen on a timescale large enough to render colloids stable for all practical purposes ${ }^{9}$.

\subsubsection{Application to Lee \& Meisel colloids}

The practical application of this theory to experiments is not straightforward, since some of the parameters (particularly $A_{\text {eff }}$ and Z) in Eq. 4.5 and 4.10 are hard to obtain accurately. By the same token, it is important to keep in mind the range of shortcomings inherent to DLVO theory:

- The approximation of $g_{i}$ to its first order term is valid only if $\beta z_{i} e \Phi(\mathbf{r})$

\footnotetext{
${ }^{9}$ In fact, already Faraday [103] noticed the metastable nature of colloidal sols, and, by the same token, some of the sols he prepared are still on display in the British Museum in London [92].
} 
is small, therefore realistic systems with large surface charges on the colloids cannot be described satisfactorily using this approximation.

- Bell et. al. [87] have shown that chloride ions replace the citrate layer, thus potentially changing the overall surface charge.

- In general, the citrate capping layer (or any other molecular surface layer) will affect the short range interaction, but is not modeled here. In the specific case studied here this may not pose a problem if the entire citrate layer is replaced by chloride ions, however, any exceedingly high analyte concentration on the surface still facilitates aggregation ${ }^{10}$.

- The colloids are modeled as perfect spheres, which is far from true for most of them, as shall be shown in Sec. 4.3.

- A whole range of intrinsic problems which are hard to pin down rigorously add to the complexity of the problem: How mobile are the charges on the colloidal surface, i.e. how polarisable are colloids? Does its charge change when approached by other charges, i.e. can dissociated molecules in the capping layer recombine with ions from the solution on demand? How do the counter-ions in the liquid arrange around colloids? How does this ion-cloud distort when colloids approach each other?

Hence, the predictions of DLVO theory are at most of a qualitative nature. Despite all these shortcomings, the DLVO approach has been widely used [104] in the past to describe colloidal dynamics, as pointed out by Boda et al. [105]:

"However, its popularity may be due as much to the ingenuity of its users in fitting parameters as to its accuracy."

\footnotetext{
${ }^{10}$ Adding $1 \mu \mathrm{M}$ Rhodamine 6G to pure Lee \& Meisel colloids will rapidly collapse the solution
} 


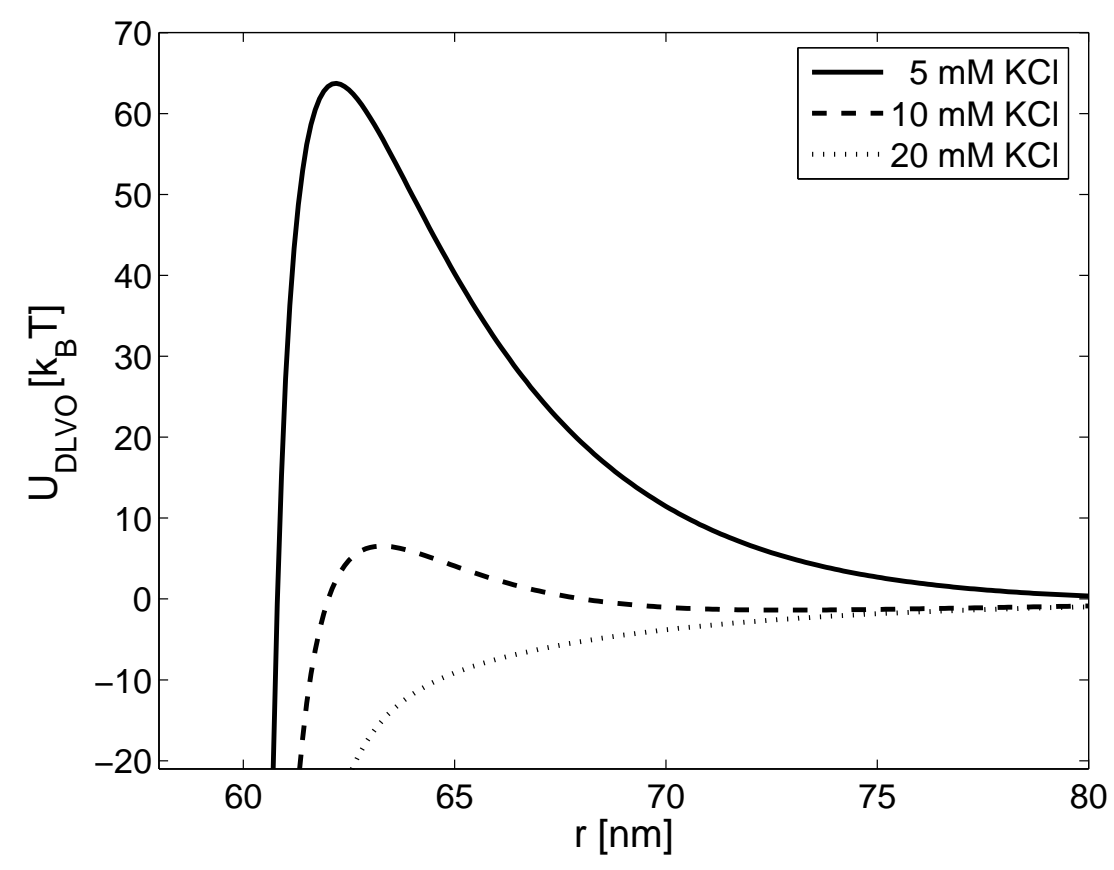

Figure 4.5 - DLVO interaction potentials in solutions of different ionic $(\mathrm{KCl})$ concentration (see legend) for Ag colloids. The following parameters were used in the calculation: radii $a=30 \mathrm{~nm}, Z=1000$, and $A_{\text {eff }} / k_{B} T=60$. In this plot, a distance of $60 \mathrm{~nm}$ means that the two spherical colloids are touching each other. Note the development of a deep negative potential very close to the "touching" condition followed by a positive repulsive barrier which is more depleted the higher the $\mathrm{KCl}$ concentration. The deep negative part of the potential means that if two particles overcome the repulsive positive barrier, they will aggregate irreversibly.

Claiming ingenuity, and for lack of a superior model, the findings of DLVO theory will nonetheless be used in the following. However, in acknowledgment of the various intrinsic problems of the theory, the DLVO potential will be evaluated only qualitatively and I shall refrain from deriving quantities (e.g. rate constants of aggregation, etc...), which would be of little significance.

Fig. 4.5 shows the DLVO potential of Ag colloids for different salt concentrations. Evidently, with increasing salt concentration, the repulsive barrier decreases. The result of an increased salt concentration is an increased screening, leaving the colloidal charge partially 'hidden'. Hence, 
the resulting Coulomb repulsion is diminished. In turn, the probability that two colloids will adhere upon impact increases, since now a larger region of the Boltzmann distribution of energies is sampled. In essence, the time scale on which aggregation takes place is decreased.

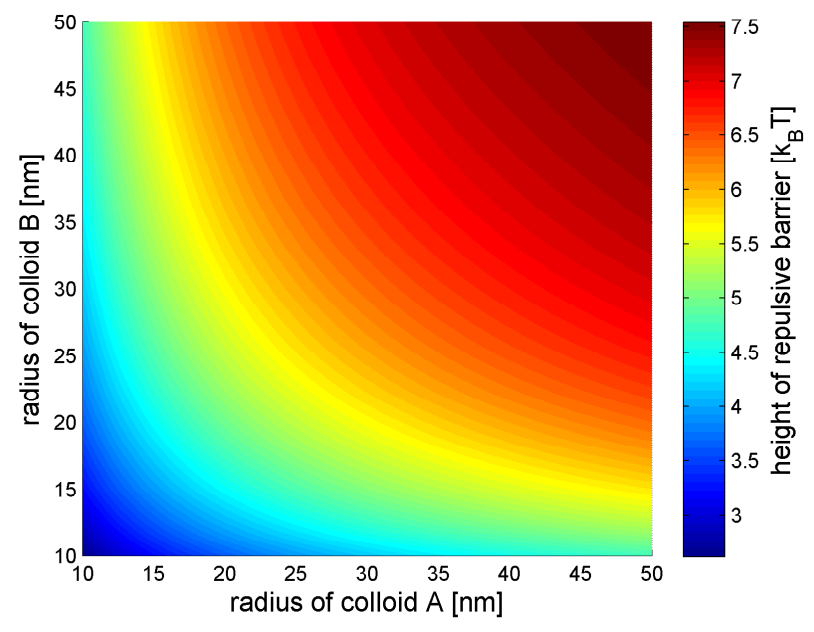

Figure 4.6 - Colour-coded map of the height of the repulsive barrier for Ag colloids of varying radii. The following parameters were used in the calculation: radii were varied from $a=10 \mathrm{~nm}$ to $50 \mathrm{~nm}$, the charge was scaled with the surface area (since it arises from the dissociated capping layer), whereas a colloid with a radius of $30 \mathrm{~nm}$ would have $Z=1000$. The Hamaker constant was kept fixed at $A_{\text {eff }} / k_{B} T=60$. What is evident here is that the repulsion increases with colloid radius. This behaviour changes for larger colloids (see Fig. 4.8).

If the salt concentration is increased sufficiently the repulsive barrier disappears completely, and the interaction potential becomes purely attractive. This is the regime of rapid coagulation in which nothing hinders the colloids from aggregating, and the rate constant of aggregation is governed only by the colloidal diffusion constant. Figures 4.6,4.7 and 4.8 explore the dependence of the repulsion and attraction on the colloid radius.

In summary, DLVO theory provides the following insights:

- The Coulomb repulsion of like-charged colloids in an electrolytic solution is partly screened, resulting in a finite repulsive barrier. 


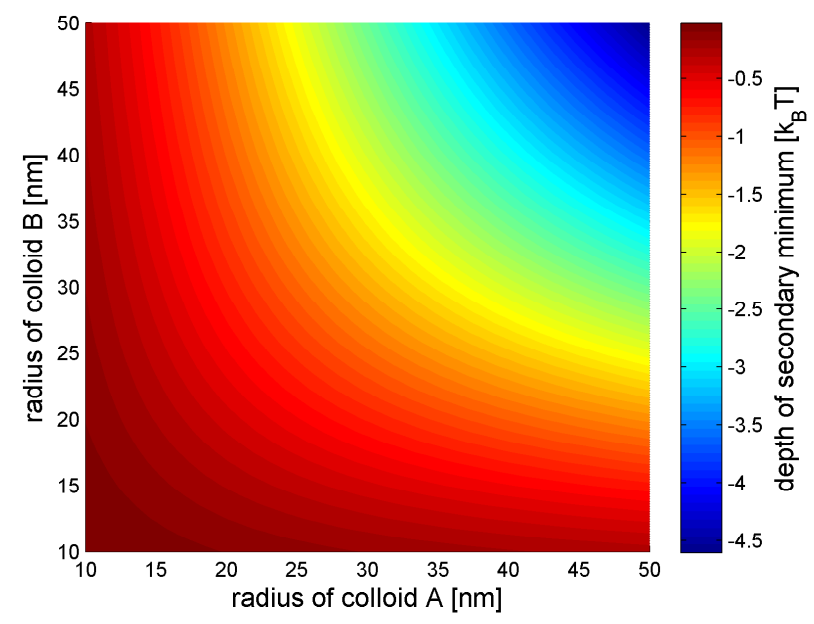

Figure 4.7-Colour-coded map of the depth of the secondary potential minimum (flocculation minimum) for Ag colloids of varying radii. The DLVO parameters are the same as in Fig. 4.6. For the average Ag colloid (i.e. $a=30 \mathrm{~nm}$ ) the minimum is relatively shallow, $\sim 1.4 k_{B} T$, causing a loose, transient association between colloids which is easily broken up (42\% of colloids have higher energies in the Maxwell-Boltzmann distribution). This interaction increases monotonically with the colloidal radii, i.e. larger colloids are stronger bound in flocculation.

- van der Waals forces provide an attractive force, which -in this casesuits to model the short-range potential minimum responsible for colloidal aggregation.

- With the Boltzmann distribution of energies it becomes clear that, unless the repulsive barrier diverges towards infinity, the colloidal sol will only be meta-stable. Thus colloidal stability becomes a question of time-scale.

- The dependence of the repulsive barrier on the ion concentration explains the experimentally observed accelerated coagulation upon salt addition. This serves as a strong hint that the mechanism which provides the enhancement upon 'activation' of the colloids is aggregate formation, rather then a more subtle chemical effect. 


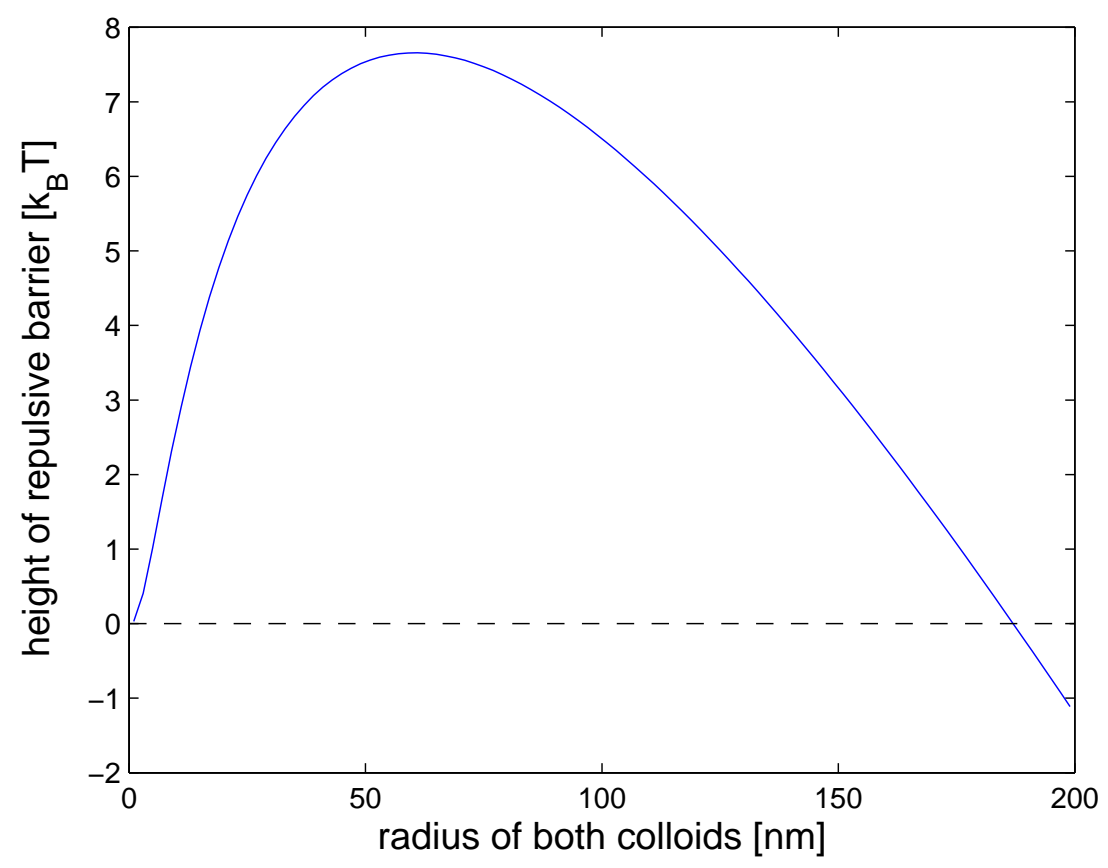

Figure 4.8 - Height of repulsive barrier as a function of colloid radius, assuming two identical colloids. The maximum occurs for particles of radius $a \approx 60 \mathrm{~nm}-$ about twice the average radius of typical Lee \& Meisel Ag colloids. Note that van der Waals forces dominate in extremely large colloids (see Fig. 4.7).

What is not explained within this framework is the apparent long-term stability after a significant salt concentration is added to the solution. As will be shown in Sec. 4.4, colloidal aggregation takes place, but subsides over time. What is stopping colloid aggregation once the coagulation threshold is lowered to within, let's say, a few $k_{B} T$ ? This remains a conundrum when considering pair-wise interactions only, but is readily explained by a many body effect. I will come back to this question in Sec. 4.5. In the next section I will show electron micrographs obtained from colloidal sols. This will, after this lengthy theoretical section, provide a first experimental characterisation of the pure colloidal system (i.e. single colloids) and of the clusters and aggregates obtained after salt addition. 


\subsection{Electron microscopy of single colloids}

For scanning electron microscopy (SEM) dry samples are required, and one therefore needs to deposit the colloidal solution onto a substrate. A common approach to do this is the procedure mentioned (in passing) by Nie \& Emory [9]: glass slides are first coated with a thin poly-l-lysine solution, which provides the base for a subsequent deposition of colloids. The general procedure for dry sample preparation for SEM is as follows:

1. Cover a glass slide in a $1 \mathrm{mM}$ aqueous poly-l-lysine solution for $5 \mathrm{~min}$.

2. Remove the poly-l-lysine solution (pipette off) and rinse with distilled water.

3. Place an aliquot of the colloidal suspension onto the slide and leave it for $1-2 \mathrm{~min}$.

4. Remove colloidal solution (pipette off), rinse with distilled water and leave to dry ( $\sim 10 \mathrm{~min})$. At this stage, the sample is ready for SERS experiments.

5. For SEM an additional step is necessary: the sample is coated with a $5 \mathrm{~nm}$ layer of platinum. This increases the quality of the SEM images by avoiding excessive charging by the electron beam.

This procedure can be applied to bare colloids, aggregated sols (sols with increased electrolyte concentration) and sols incubated with analytes (see below). The polylysine coating on the glass acts as a "glue" that captures a small fraction of the single particles or clusters existing in the colloidal solution and immobilises them for further inspection once the sample is dry. This collection of captured clusters is expected to be a faithful representation of the situation in the liquid, accordingly. In short, 'snapshots' of the solution are taken. This procedure avoids the possibility of additional aggregation, which typically would be induced if the colloidal solution was left to dry completely. 
However, achieving a representative sampling of the particles/clusters poses an optimisation problem - the question being: What is the optimal time the polylysine coated substrate should be exposed to the solution for? The longer the time, the larger the number of particles/clusters that adhere to the surface. Initially, one might expect that to be beneficial to the experimental statistics. However, as the surface coverage increases, the probability of accidental clustering increases as well. It was found that a one minute exposure followed by rinsing seems to be a good compromise; this results, in the case of bare Lee \& Meisel Ag colloids, in a substrate with an area density of $\sim 1$ particle per $1 \mu \mathrm{m}^{2}$, of which only very few $(\sim 5 \%)$ are small aggregates.

Applying simple geometry, the probability of accidental clustering can be estimated from the surface coverage. The average colloid has a surface footprint

$$
A=4 \pi(30 \mathrm{~nm})^{2} \approx 10^{-2} \mu \mathrm{m}^{2}
$$

and -ignoring any interaction- if a colloid falls into an area of $0.045 \mu \mathrm{m}^{2}$ an aggregate will result ${ }^{11}$. Since we observe (see below) roughly one colloid per $\mu \mathrm{m}^{2}$, the estimated chances for accidental aggregation are $~$ $5 \%$. This is in good agreement with the observed cluster density in the SEM images of the bare colloidal sol. In an ideal world, one could now conclude that all observed clusters must be of accidental nature; however, the colloidal surface density is a statistically weak result, since it was estimated from only a few SEM images. Moreover, repulsive and attractive forces which govern the aggregation dynamics in solution (see Sec. 4.2) have been completely neglected here. It remains an open question what role the DLVO potential plays during colloidal adhesion to a surface.

\footnotetext{
${ }^{11}$ The center-to-center distance of touching colloids is $2 a=60 \mathrm{~nm}$, thus the area within which a colloid must happen to fall to accidentally aggregate is $4 \pi(60 \mathrm{~nm})^{2}=0.045 \mu \mathrm{m}^{2}$.
} 


\subsubsection{Investigated samples}

To investigate possible aggregation pathways, different mixtures stages were deposited:

pure colloids - pC This is the standard Ag colloid solution obtained from the Lee \& Meisel recipe, without any additives.

pC with salt - CK Here $10 \mathrm{mM} \mathrm{KCl}$ was added to the bare colloidal sol to increase the electrolyte concentration, and thus induce aggregation.

CK with dye - CKB Furthermore, $200 \mathrm{nM}$ BTZ2 (see Sec. 2.3) was added to the CK solution. This concentration leads to an estimated $\sim 1000$ molecules per colloid ${ }^{12}$. This number will obviously fluctuate greatly due to the polydispersity (see Fig. 4.9) of the colloids. BTZ2 was added to investigate whether the analyte (at a typical concentration for SERS) could have any influence on the aggregation dynamics, since it is well known that high concentrations of dye, e.g. $1 \mu \mathrm{M}$ Rh6G, will alter the surface/bulk chemistry sufficiently to rapidly coagulate the sol. In Sec. 4.4 this sample (in its liquid form) will be used to monitor the aggregation dynamics in-situ.

\subsubsection{Single colloids and dimers}

Figure 4.9 shows micrograph excerpts of $\mathbf{p C}$. As expected from the bare Lee \& Meisel sol, mostly single colloids are present, accompanied by a small fraction of dimers. In Fig. 4.10 micrographs of colloidal dimers are shown. Although these aggregates can be found already in the $\mathrm{pC}$ sample, they predominantly occur in CK. These dimers are the classical example of a SERS substrate with a high enhancement; as was shown in Sec. 3.1.5,

\footnotetext{
${ }^{12}$ A dye concentration of $200 \mathrm{nM}$ corresponds to $1.2 \cdot 10^{14} \mathrm{dyes} / \mathrm{cm}^{3}$. The colloid number density of $\rho \approx 10^{11} \mathrm{~cm}^{-3}$ then corresponds to an average of $\sim 1000$ dyes/colloid (assuming complete adsorption, i.e. all molecules adsorb to the surface, the supernatant becomes dye-free).
} 
this geometry is expected to yield a hotspot confined in the gap between the colloids.

\subsubsection{Aggregates - small and large structures}

For samples CK and CKB an estimated one third of the colloids had aggregated, forming small clusters of various types. The large range of cluster sizes observed here, can be attributed to the poly-dispersity of the colloids in the starting solution. Note that CKB shows no marked difference to CK, the aggregational state is the same in both samples. Since the addition of BTZ2 does not appear to induce a qualitative difference, one can infer that, at $200 \mathrm{nM}$ dye concentration, the overall balance of charge is not dominated by the dye but rather by the salt ions and the capping citrate layer. 


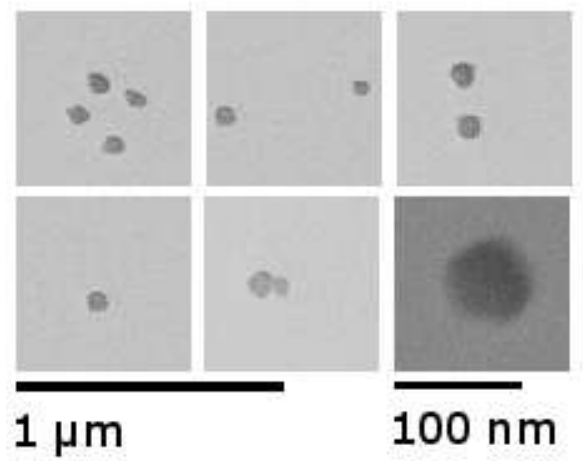

Figure 4.9 - Excerpts from SEM images of pure colloids (pC) at various magnifications. Note that for $\mathbf{p C}$ a few dimers can be found, although they are rather rare $(\sim 5 \%)$. The single Ag colloids match very well the average expected size deduced from dynamic light scattering (DLS), which is $\sim 60 \mathrm{~nm}$ in diameter. The bottom right image shows one colloid in full magnification, note the additional length-scale.

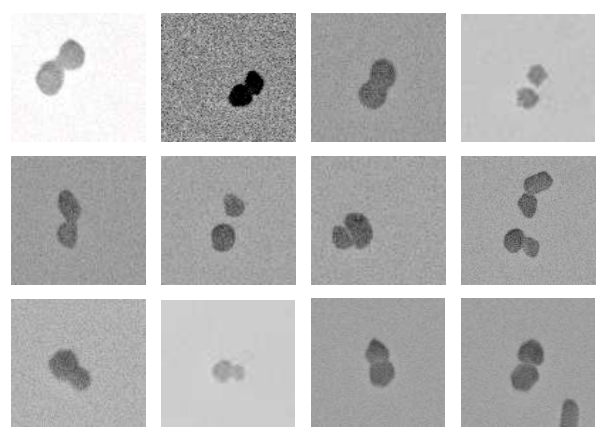

Figure 4.10 - SEM image excerpts of colloidal dimers. This type of aggregate is common in CK (see text for details). It is the archetypal example of a colloidal 'hot particle', which, given a suitable geometrical arrangement and field alignment [66, $61,63,62]$, is believed to be capable of providing enhancements sufficient for single molecule observation [66, 27]. 

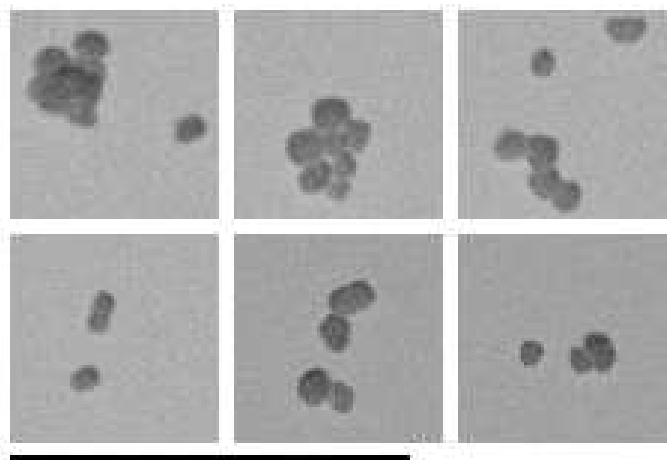

\section{$1 \mu \mathrm{m}$}

Figure 4.11 - SEM image excerpts of small colloidal clusters obtained from dried CK/CKB solutions. This type of aggregate is common in CK and CKB. There is no noticeable difference in the substrates obtained from CK or CKB - the analyte dye has no measurable effect on the aggregates observed in the SEM (see text for details). Structures of this type will in general contain multiple hotspots, each with a polarisation dependent coupling [61]. Thus a rotation of the polarisation plane will cause a different redistribution pattern of the laser field, emphasising different regions in the cluster. This effect is potentially important in Bi-Analyte SERS (see Sec. 5.3.1). 


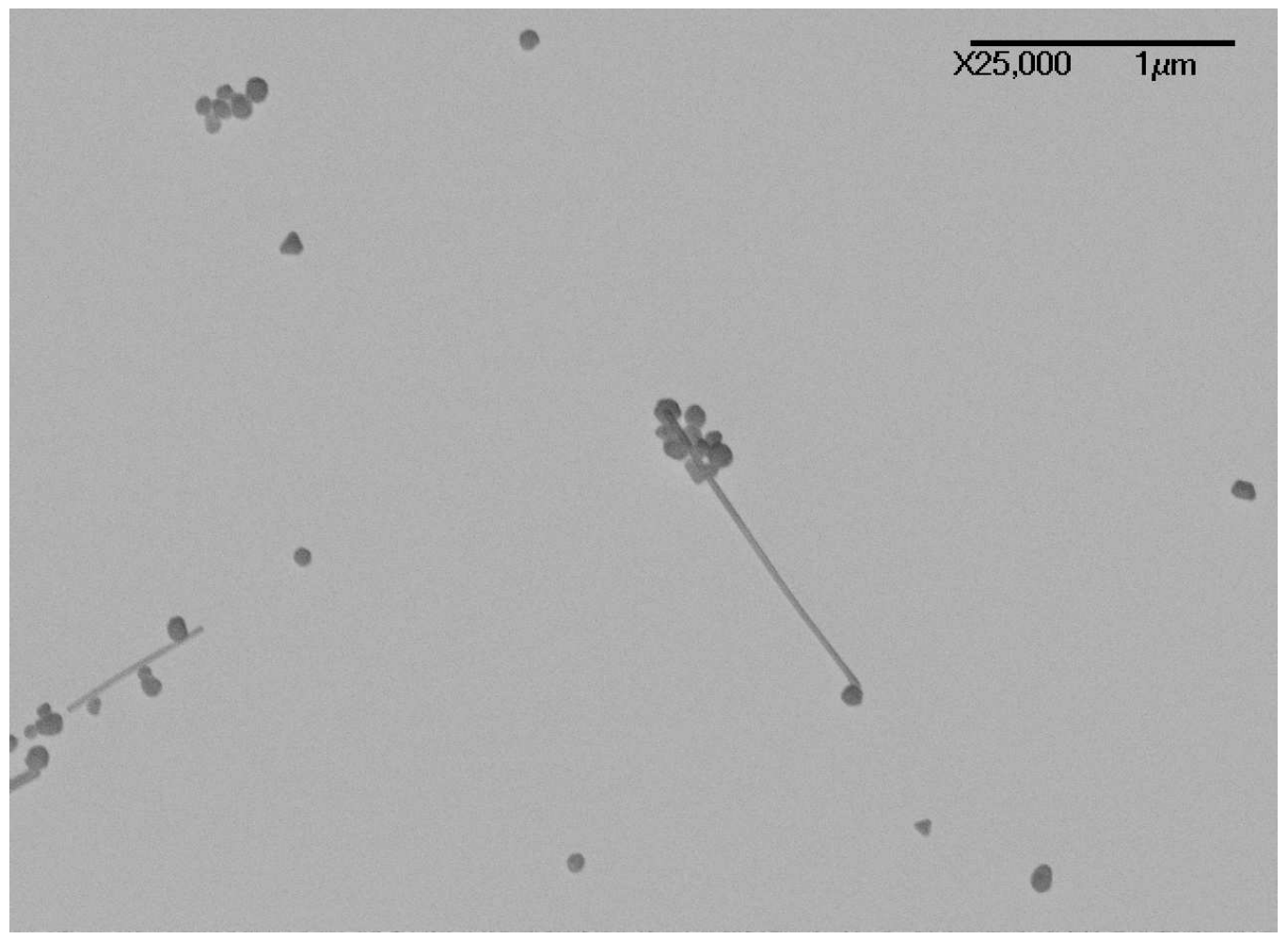

Figure 4.12 - SEM image of a $(5 \times 4) \mu \mathrm{m}$ region on a dried $\mathrm{CK} / \mathrm{CKB}$ solution containing various types of particles. There are seven single particles, two of which are triangular, and three aggregates, two of which contain rod-shaped particles. The central rod is about $1 \mu \mathrm{m}$ long and $\sim 20 \mathrm{~nm}$ thick. Colloids seem to tend to stick to the rods, as many examples of this type were found. These rods may be the product of direction-dependent aggregation followed by partial melting (also see Fig. 4.13 and text for details). 


\subsubsection{Rod-like colloidal structures}

One interesting, yet little investigated observation in the SEM images of aggregated colloids is the appearance of rod-like structures. These can be found in the bare Lee \& Meisel colloidal sol, but are rare and seem to be the product of the altered chemistry and aggregation state in the CK solutions. There is a simple and compelling argument for the formation of these rods, yet much of it remains speculative. The underlying physics which presumably govern this effect are discussed below; the proposed mechanism is described as part of Sec. 4.5. Nonetheless, a brief outline is justifiable here: colloidal aggregation may prefer the formation of rod-like structures because the repulsive barrier of an aggregate has an angular dependence (as opposed to the repulsion from a single spherical colloid, which is isotropic). The subsequent melting and rod-formation may be driven by surface tension. This is likely to happen already during synthesis, but, so far, firm evidence (i.e. abundant occurrence in SEM images) has only been gathered for the case of $\mathrm{KCl}$-aggregated colloids.

The formation of such a rod from a linear arrangement of colloids has not been directly observed, currently only disconnected SEM snapshots of what seems to constitute different stages of this transformation have been taken (see Fig. 4.13). It is important to keep aggregational 'outliers' like this in mind when it comes to the discussion of intensity fluctuations in SERS; rare, high-intensity events could be produced by this type of particle. 

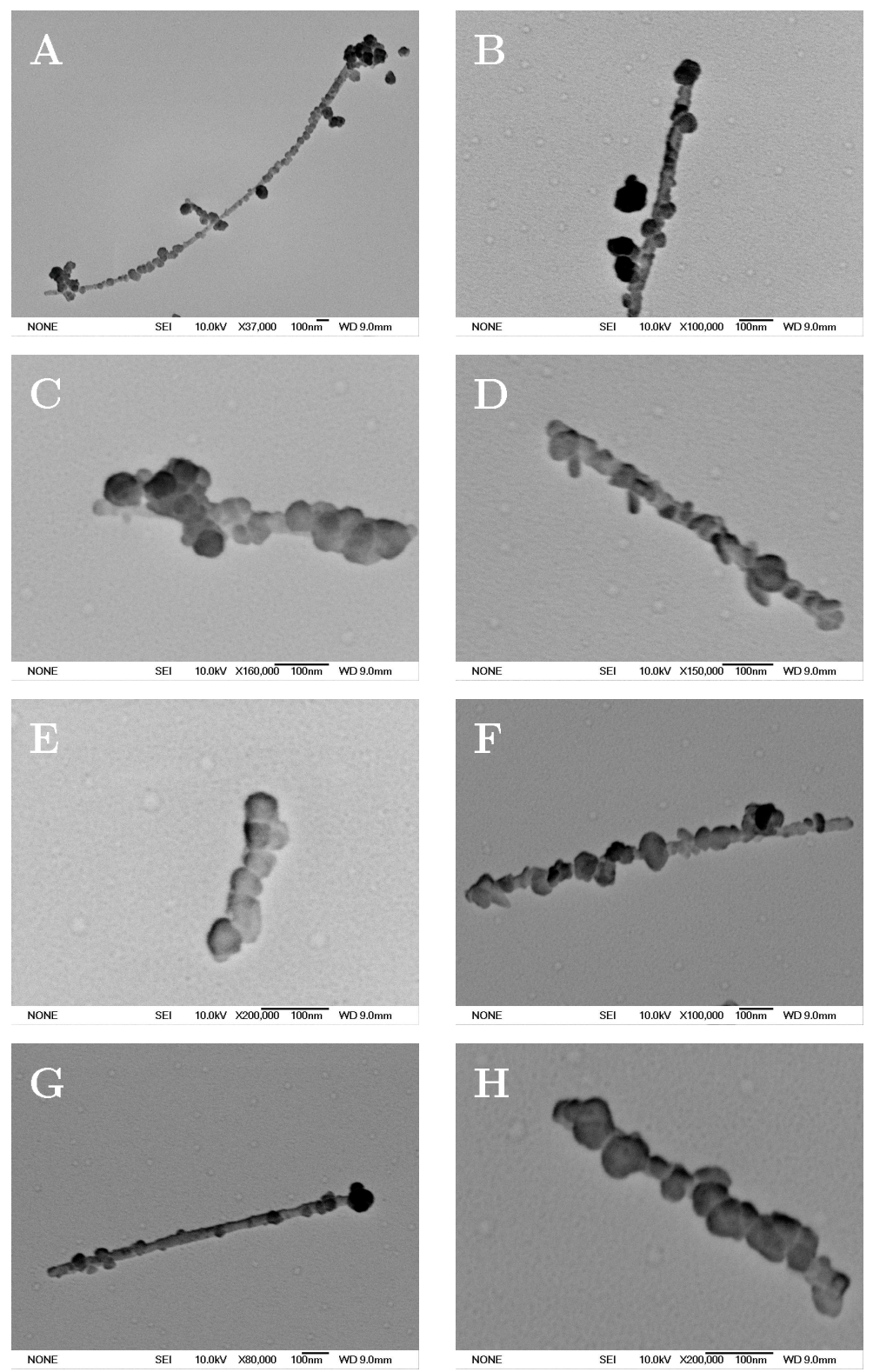

Figure 4.13 - SEM image excerpts at various magnifications of rod-like aggregates found on in CK and CKB. The rods shown here vary in length between $250 \mathrm{~nm}(\mathbf{C})$ and $3.4 \mu \mathrm{m}(\mathbf{A})$. Note that most rods are not a single particle, but rather a line-up of single colloids into a rod $(\mathbf{C}, \mathbf{E}, \mathbf{H})$, with some structures appearing to show partial melting $(\mathbf{A}, \mathbf{D}, \mathbf{F})$. 


\subsection{In-situ observation of colloidal aggregation}

From the electron micrographs it is clear that, when $\mathrm{KCl}$ is added, aggregation occurs in the colloidal sol. To confirm what em calculations predict, i.e. that colloidal aggregation is linked to the SERS intensity (via the presence of hotspots in aggregates), SERS experiments with in-situ $\mathrm{KCl}$ addition were carried out. In layman's terms, the latin phrase 'in-situ' translates into the following simple procedure: during the measurement of a time-series of SERS spectra, $\mathrm{KCl}$ is added to the sample.

For the in-situ measurements SERS-active solutions were prepared by adding $200 \mathrm{nM}$ of BTZ2 (see Sec. 2.3) to Lee \& Meisel colloids. Unless stated otherwise, the number of dye molecules per colloid is $\sim 1000$.

\subsubsection{The build-up and origin of SERS signals in liquid}

The first task is to confirm the origin of the SERS signals in liquid: single colloids or aggregates? Clearly, a large body of evidence points at aggregates:

1. It is well-known that the addition of a salt such as $\mathrm{KCl}$ is necessary to observe strong SERS signals.

2. DLVO theory predicts the stabilising electrostatic barrier to diminish upon salt addition (and eventually vanish completely for sufficiently high salt concentrations.)

3. Electron micrographs attest the presence of aggregates in colloidal sols after salt addition.

Despite this evidence, it was considered possible that $\mathrm{KCl}$ has a strong chemical effect [83], i.e. that chloride ions modify the silver surface in such a way that SERS signals are more enhanced (see discussion on the 
notion of chemical enhancement, Sec. 3.2). This could occur without colloid aggregation.

If SERS comes from aggregates forming after $\mathrm{KCl}$ addition, the following effects should be observable:

Build-up of the signal: Measuring a sample with analyte (BTZ2), a gradual build-up of the signal (as aggregates form) is expected to occur after the point of $\mathrm{KCl}$ addition.

Colloid concentration dependence: Since colloids need to collide to form aggregates, and are assumed to undergo Brownian motion, one expects the rate of aggregate formation to depend on the colloid concentration, for particles should take longer/shorter to find each other depending on their number density. This should be reflected in the time-dependence of the SERS intensity. Such a colloid concentration dependence is not explainable with the chemical effect.

Dimer formation: The initial stages of the build-up ought to be well represented by the dynamics of dimer formation only, which is the most probable event at the onset of aggregation, since the overall number density and mobility decreases with continuing aggregate formation.

To study the build-up of the SERS signals as a function of colloid concentration we carried out in-situ measurements. The experimental approach is as follows:

1. The base colloid solution was put in a small $(\sim 1 \mathrm{ml})$ disposable sample holder and a SERS time-series measurement in immersion was started (1 sec integration time) with the CCD detector conveniently centered in a region that would allow the spectrum of the dye to be monitored later.

2. After a few minutes (which yield the background SERS signal of the bulk solution), BTZ2 was added. BTZ2 is known to adsorb strongly 
to Ag-colloids by forming a covalent bond. As confirmed by the electron micrographs (see Sec. 4.3), at the concentration used here this dye does not noticeably induce colloidal aggregation. The addition of BTZ2 provides the (weak) SERS signal without aggregation (single particle enhancement regime).

3. Finally, a $\mathrm{KCl}$ solution was added to achieve the desired full SERS condition $\left(c_{\mathrm{KCl}}=10 \mathrm{mM}\right)$. This $\mathrm{KCl}$ concentration corresponds to about half of the concentration required for fast coagulation of the colloids; it pushes the colloidal sol towards instability, as the repulsive forces between colloids are reduced (see Sec. 4.2).

4. Further Raman spectra were recorded for up to 2 hours at intervals (dead time) of 1-1.5 sec.

Three such in-situ measurements, based on bulk solutions with different colloid concentrations $c$ were carried out:

sample A: $\hat{c} \approx 10^{11} \frac{\text { colloids }}{\mathrm{cm}^{3}}$,

sample B: $c=\hat{c} / 2$, and

sample C: $c=\hat{c} / 4$.

Figure. 4.14 shows the average SERS signals over several minutes in the steady state of the three situations (colloid, colloid+dye, colloid + dye $+\mathrm{KCl}$ ). Note the logarithmic scale in the figure and also note that SERS signals, although weak, can be seen in the liquid before the induced aggregation.

The dynamics of the build-up of the signal is explicitly shown in Fig. 4.15, where a floating average (window size: 50 spectra) of the spectrally integrated Raman intensities of samples A, B and C -starting from the point of $\mathrm{KCl}$ addition- are plotted.

The first step of aggregation is two colloids forming one dimer; a process that will run with a rate constant $k$ which depends on the positive potential barrier (Fig. 4.5). Since two colloids need to be present in the same 


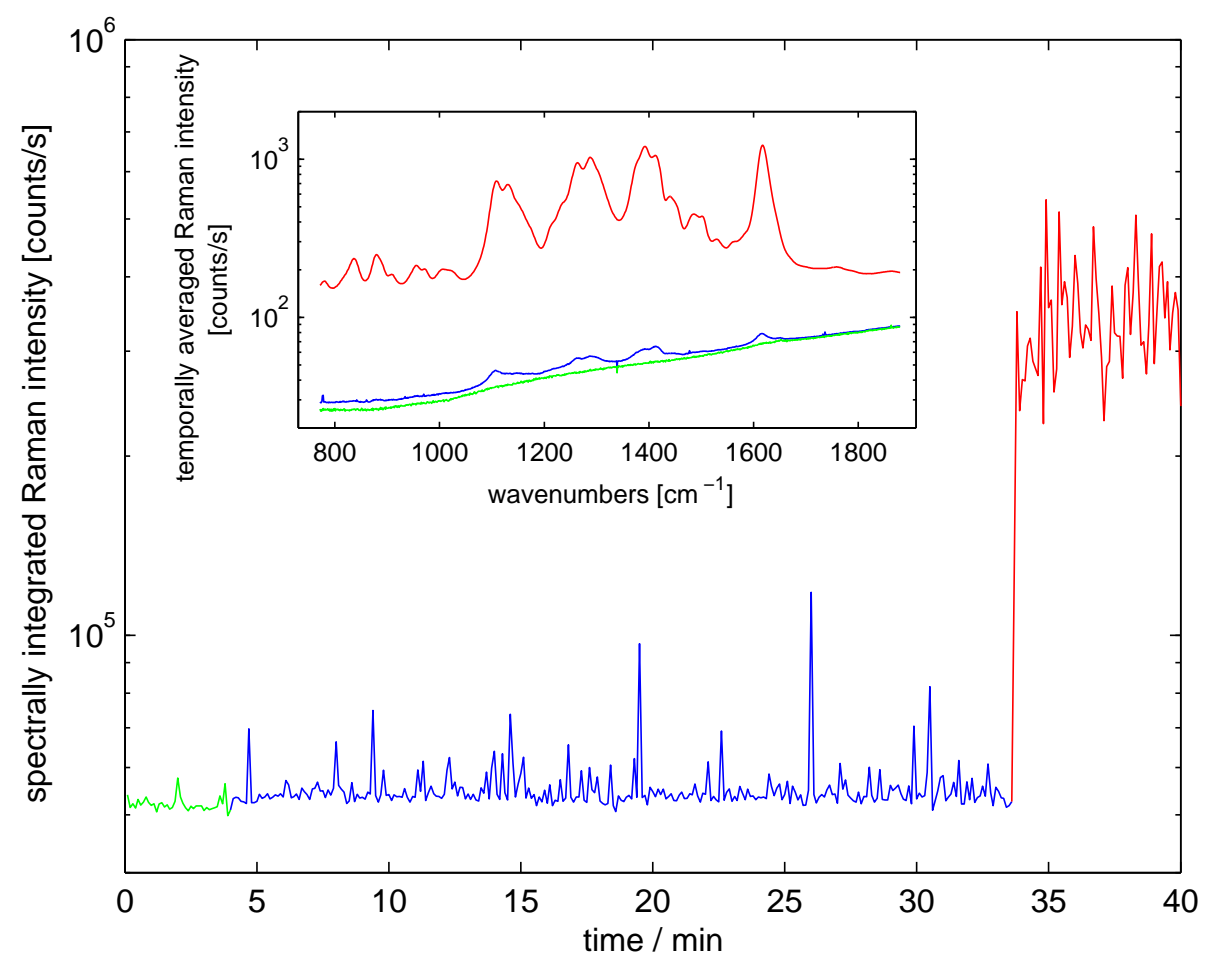

Figure 4.14 - Averaged Raman/SERS spectra of sample C during the three insitu stages: pure colloid solution (background; green line, average of 150 spectra in the inset), after addition of BTZ2 but without $\mathrm{KCl}$ (blue line, average of 750 spectra), and after addition of $\mathrm{KCl}$ (red line, average of 2300 spectra). Note the log-scale on the intensity axis.

place, the process depends on the squared concentration of single colloids $c_{S}$. Accordingly, the dimer concentration $c_{D}$ will rise at half the rate that $c_{S}$ falls (to ensure colloid number conservation), i.e.:

$$
\frac{d c_{S}}{d t}=-k c_{S}^{2}, \quad \frac{d c_{D}}{d t}=\frac{k}{2} c_{S}^{2}, \quad c_{S}+2 c_{D}=c_{0},
$$

where $c_{0}$ is the initial single colloid concentration. The solution of the differential equations leads to an expression for the time dependent dimer concentration, to wit:

$$
c_{D}(t)=\frac{c_{0}}{2}\left(\frac{c_{0} k t}{c_{0} k t+1}\right) .
$$




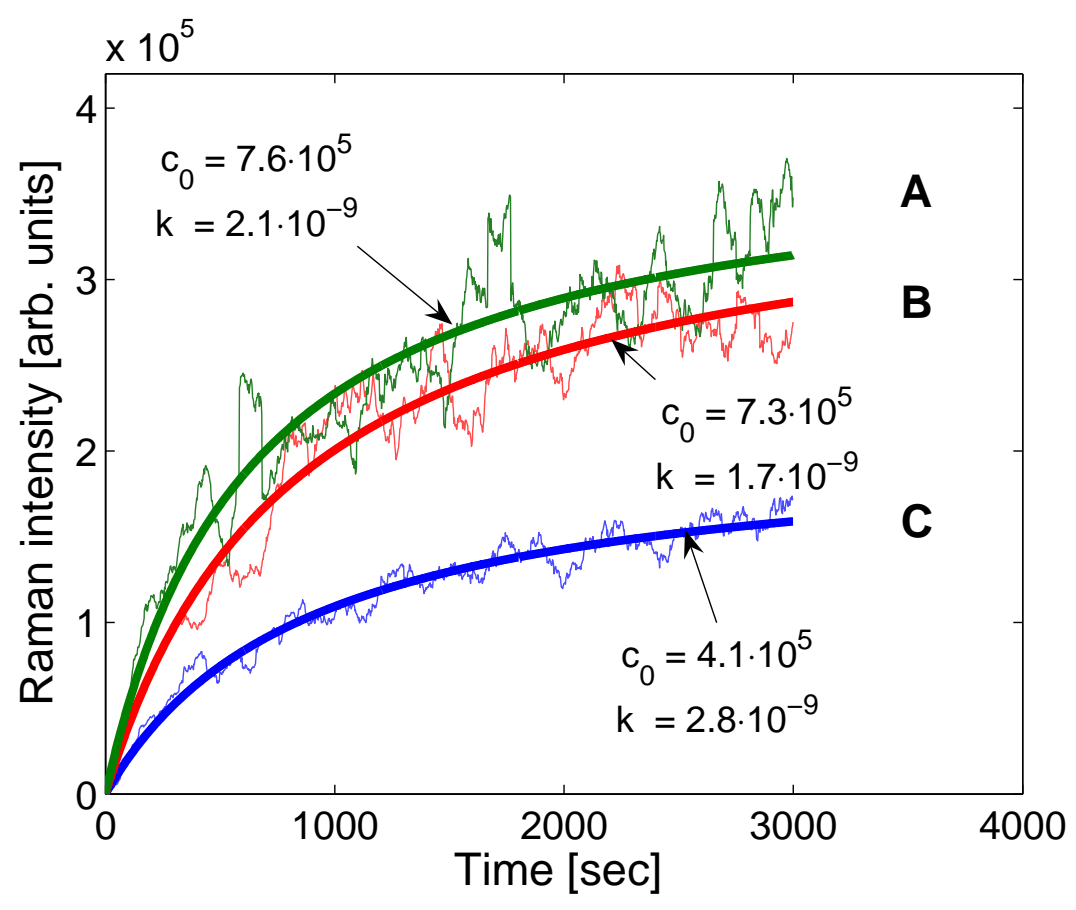

Figure 4.15 - Build-up of the SERS signal (floating average with 50 spectra window size) for the samples with different initial colloid concentrations. Fits of Eq. 4.16 with the corresponding parameters are shown. A very good representation of the build-up is achieved even for sample $\mathbf{A}$, where clustering beyond simple dimers in this time scale is expected to occur. See the text for further details.

Given the enormous SERS enhancement expected from a dimer (see Sec. 3.1.5), and neglecting the possible formation of higher aggregates, one can assume that $I_{\text {Raman }} \propto c_{D}$, i.e. this function is expected to be a good approximation to the Raman-signal build-up, at least for low colloid concentrations.

In Fig. 4.15, least squares fits of Eq. 4.16 are shown (thick solid lines) and the corresponding parameters displayed. The fits yield nearly equal rate constants $k$ for the three different concentration regimes - this is expected since $k$ only depends on the interaction potential, which is identical for samples $\mathbf{A}, \mathbf{B}$ and $\mathbf{C}$ (same $c_{\mathrm{KCl}}$ ). The fitted initial concentrations $c_{0}$ nearly reproduce the expected factor of 2 between samples $\mathbf{B}$ and $\mathbf{C}$, but start having problems with the scaling for sample $\mathbf{A}$. This is understandable, since the system of equations (4.15) is expected to be less and less accurate the 


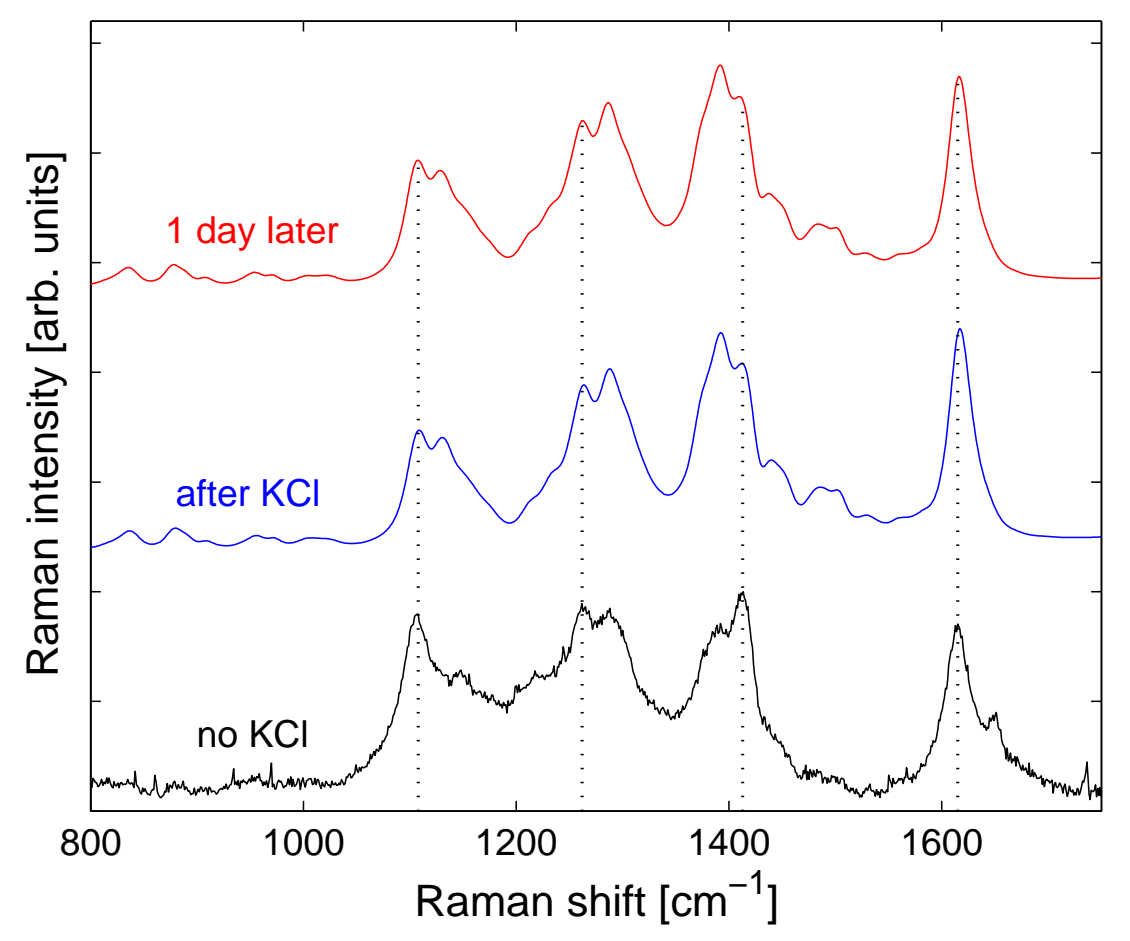

Figure 4.16 - Average Raman spectra of the various stages in the in-situ experiment. All spectra are normalised and displaced to ease comparison. Shown are the average spectra before addition of $\mathrm{KCl}$ (black line; mean of 330 spectra, $30 \mathrm{~min}$ ), after addition of $\mathrm{KCl}$ (blue line; mean of 2000 spectra, $200 \mathrm{~min}$ ) and after one day (red line; mean of 600 spectra, $60 \mathrm{~min}$ ). This composition highlights the differences in the relative peak intensities seen in the three aggregation regimes. Evidently, minor spectral modifications occur upon salt addition, but no further modification takes place for longer incubation times. The similarity of the spectra lends plausibility to the em effect as the main cause of the enhancement. See text for details. 
higher the concentration, for it fails to consider further clustering beyond dimers (i.e. trimer, tetramers, etc...). This 'reduced' initial concentration $c_{0}$ for sample A also becomes understandable in light of the enhancement expected from such higher aggregates: the em enhancement depends on the hotspots present in these aggregates, which, due to their high selectivity with respect to the various conditions of the exciting field [61], are neither in overall strength nor individual number proportional to the number of colloids constituting the aggregate. Simply put, an aggregate of 10 colloids will, in general, emit less than five times the intensity of a dimer.

Extending on the same data, the in-situ strategy also yields an argument of plausibility concerning the discussion around chemical enhancements. Fig. 4.16 shows the average Raman spectrum of BTZ2 in the colloidal sol before $\mathrm{KCl}$ addition (black), after $\mathrm{KCl}$ addition (blue) and after one day (red). If a chemical effect, i.e. a charge-transfer complex, was responsible for the enhancement following $\mathrm{KCl}$ addition, then one would expect a strong dependence on the vibrational mode [106]. This is, as is evident from the plot, not the case. Although the minor modifications of relative intensities seen in the spectra may be due to a more electronic effect, the results are equally understandable invoking $\mathrm{em}$ enhancements and aggregation: As noted in Sec. 3.1.5, the variations in relative intensities of modes are a consequence of the local field in hotspots.

\section{Concluding remarks}

In conclusion, it is quite clear that the dynamics of clustering -after screening of the Coulomb barrier- can be followed to a relatively good degree of accuracy both experimentally and theoretically. In the final steady state we achieve colloidal suspensions which are highly SERS active, have a limited amount of clustering, and survive for several months without any deterioration in the signal level and concentration of clusters (an effect which will be addressed in the next section). This agreement strongly supports the fact that SERS signals originate mainly from clusters, with a 
dominant em enhancement, as opposed to single particles profiting from a large chemical enhancement. A noteworthy caveat is that, although the existence and effect of the aggregates is proven beyond doubt, the exact nature of the clusters, however, cannot be decided by this experiment. Of particular interest for the present discussion is to clarify if the clustering is permanent (coagulation), temporary (reversible aggregation or flocculation), or an even more dynamic pairing in which particles briefly approach each other close enough to produce a large SERS enhancement, but never actually 'bind' with each other. I will come back to this issue in chapter 5 .

In an auxiliary note, it is worth mentioning that the presence of these clusters does not have a dramatic, or sometimes even measurable, effect on the overall absorption spectrum of the solution. The absorption spectrum is thus 'blind' towards aggregates, and therefore does not allow quantification of the colloidal state of aggregation. Only if the state of aggregation of the colloid is vastly increased (e.g. by increasing the salt concentration), a wide, shallow absorption band emerges at long wavelengths; as reported, for example, in Ref. [107]. This only happens, however, well beyond the metastable phase which is of interest here. It is a demonstration that, in general, the absorption spectrum is not a good measure of where the Raman enhancement will occur; a topic that has been studied in detail in Ref. [108].

Figure 4.17 provides a lead to the next topic: plotted are the histograms of Raman intensities (summed over the full spectrum) pertaining to the time-series of spectra acquired at various intervals after $\mathrm{KCl}$-addition. For the first and second 100 minutes of progressing aggregation, notably, the histograms (blue and light blue lines) are near-identical, and although the average intensity (dotted lines) increases with time, it does so by less than $5 \%$ within the given time. The same sample, on the next day (red line), exhibits a more progressed aggregation: the average has increased by roughly $25 \%$, and intensities are generally more spread out. Note that the sudden rise at the highest intensity (present for the later two measurements) is an artifact from binning; it merely indicates that there 


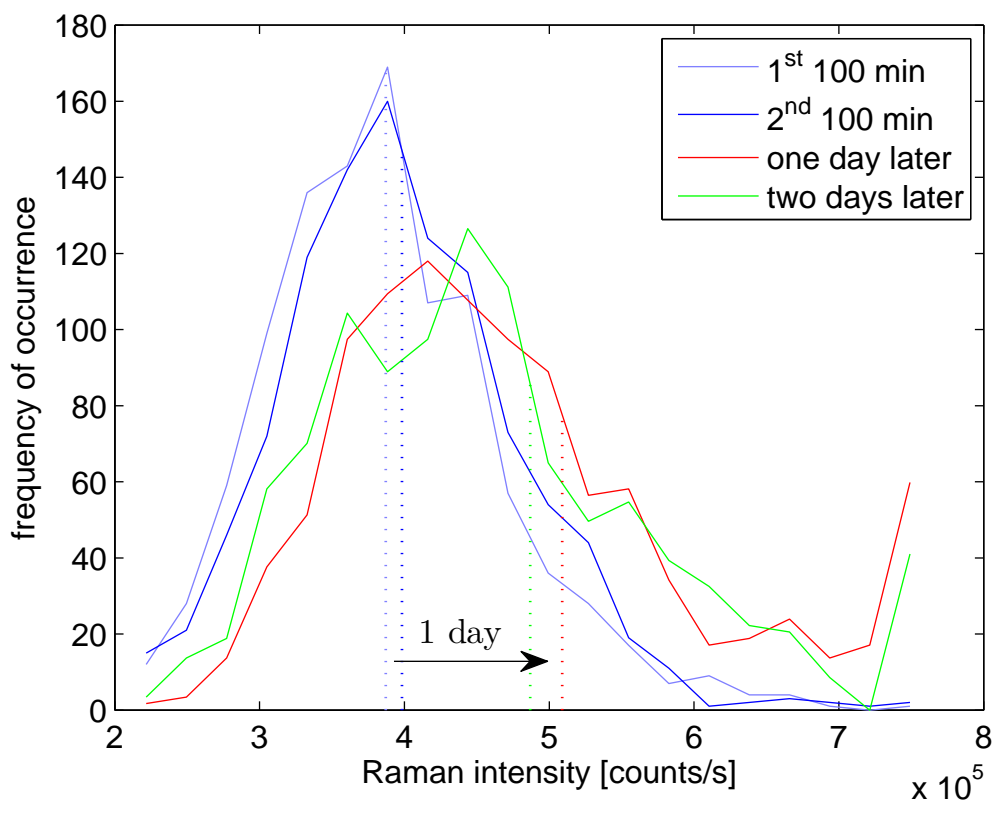

Figure 4.17 - Evolution of colloidal aggregation shown via histograms of the integrated Raman intensities from: $1-100 \mathrm{~min}$ after $\mathrm{KCl}$ addition (light blue), $101-200 \mathrm{~min}$ after $\mathrm{KCl}$ addition (blue), after one day (red) and after two days (green). The first two intervals are spanned by 1000 spectra each (integration time $5 \mathrm{~s}$, dead-time $1 \mathrm{~s}$ ), the latter two measurements are comprised of $600 \mathrm{spec}-$ tra (corresponding to a $1 \mathrm{~h}$ period) each. For ease of comparison, the histograms of the later measurements were scaled up by a factor of $5 / 3$ to correct for the number of spectra. Average integrated intensities (dotted lines) are shown. See text for details. 
are a number of cases (50-60) in which the intensity is at least as large as the highest shown here. Markedly, two days later (green line), the same sample does not appear to have undergone further aggregation; the histogram is of a similar shape as the day before, and more importantly, the average intensity is roughly the same as before. Did aggregation stop, or slow to unnoticeable levels? This thought is backed by the more general observation of the longevity of SERS samples: even months after preparation and induction of aggregation via $\mathrm{KCl}$, these samples still show strong SERS activity and little sign of coagulation. I will dedicate the next section to properly address the mechanism responsible for this unexpected stability. 


\subsection{Monte Carlo simulations}

The experimental finding of gradually decelerating aggregation and, thus, long-lived SERS active liquid samples motivates a question: What governs the aggregation dynamics of colloidal particles? Is it understandable simply in terms of the decreasing number density of colloids as aggregation progresses? What is the pair-interaction potential between a single colloid and an aggregate?

\subsubsection{Formulating the many-body problem}

A qualitative answer to the dynamics governing the interaction between a pair of single colloids was given through DLVO theory, in Sec. 4.2. However, the intuitive pair-wise interaction alone cannot explain what is observed: once the repulsive potential is low enough to allow aggregation, what prevents it to continue until complete coagulation?

\section{Colloid-colloid interactions}

To re-iterate, one can differentiate between the following DLVO-predicted regimes (compare Fig. 4.5):

- If the potential barrier is high compared to the thermal energy, $V_{\text {barrier }} \gg k_{B} T$, the system is practically stable, i.e. meta-stable for many years. Brownian motion of colloids virtually never overcomes the repulsive barrier.

- For a barrier comparable to $k_{B} T$, one expects the life-time of the metastable sol to drop significantly; e.g. for $V_{\text {barrier }}=2 k_{B} T, 26 \%$ of all colloid-colloid interactions are predicted to overcome the barrier.

- In rapid coagulation situations, i.e. when the repulsive barrier has vanished completely, $V_{\text {barrier }}<0$, it is assumed that the aggregation 
process is mainly driven by Brownian motion; there is no potential barrier for collisions.

In conclusion, from DLVO theory, the aggregation rate can be derived to be a function of the ratio $V_{\text {barrier }} / k_{B} T$ and the rate of collisions (related to the diffusion constant) which, implicitly, depends on the number density of colloids $n_{\text {colls }}$. The monotonic decrease in $n_{\text {colls }}$ as colloidal dimers are formed is a first hint towards a mechanism limiting aggregation; it raises the question: how do colloids interact with aggregates of colloids?

\section{Colloid-aggregate interactions}

Once two colloids have formed a dimer, what forces does this new entity exert upon an approaching third colloid? To approximate the colloidaggregate interaction potential, it is instructive to review the two main contributions in the DLVO potential, and what change thereof is to be expected as colloids form aggregates:

- The repulsive barrier is due to the like-charged colloids. Assuming the charge of an aggregate to be the sum of the charges of the constituting colloids, it becomes clear that the potential height of the repulsive barrier is proportional to the number of colloids in an aggregate. This makes it incrementally harder for additional colloids to approach an aggregate, and hence introduces an aggregation-limiting mechanism through Coulomb-blocking. Furthermore, by assuming immobile surface charges, the overall potential barrier is closely approximated by the spatial sum of the interaction potentials of the colloids; this breaks the symmetry in the interaction, as it is now 'easier' for a like-charged colloid to approach a dimer along its axis, rather than perpendicular to it. This is a possible explanation for the tendency of aggregates to form rod-like shapes (see Sec. 4.3.4).

- The van der Waals/Casimir attraction strongly depends on the distance between two interacting surfaces. Thus, between an aggregate 
and a colloid, it remains essentially unchanged, as the effectively interacting surfaces do not significantly change. Note however, that the effect may gain strength in aggregate-aggregate interactions, as the effective surface of interaction may (depending on shape and relative orientation) be significantly increased.

These considerations imply that forming a cluster with two particles might be feasible if the barrier is a few $k_{B} T^{\prime}$ 's, but it will become less and less probable (exponentially) to add an additional particle to the cluster. In this context, it becomes self-evident that aggregation dynamics, and hence any self-limiting effect, arise from a many body problem.

\section{The model 'world' and the model potential}

With these considerations in mind, it is now a simple matter of abstraction to formulate a suitable model potential for the Monte Carlo (MC) simulation. The aim is to simulate and follow the aggregational dynamics of particles with a DLVO-like potential, whereas said potential is assumed to be close to the coagulation threshold, i.e. $V_{\text {barrier }} \approx k_{B} T$.

The 'world' in which the colloidal interactions will be simulated is a twodimensional, finite, discrete, cartesian grid of $200 \times 200$ points. This is fully sufficient for the qualitative conclusions drawn below, and, above all, reduces the computational cost to a manageable level. A colloid becomes a dimensionless dot in this world, occupying a single point in the lattice. An aggregate, thus, is comprised of at least two colloids occupying neighbouring lattice sites. The boundaries of the lattice are closed, i.e. particles cannot move past them. It becomes clear that, by nature of the model world, distance is expressed in discrete lattice-units and the interaction potential must take a discrete shape, as the colloids move in a stepwise fashion from one site to another. Fig. 4.18 shows the abstraction of the DLVO potential into a simple, discretised pair-interaction potential for the MC simulation. This potential mimics the features of the DLVO potential: 

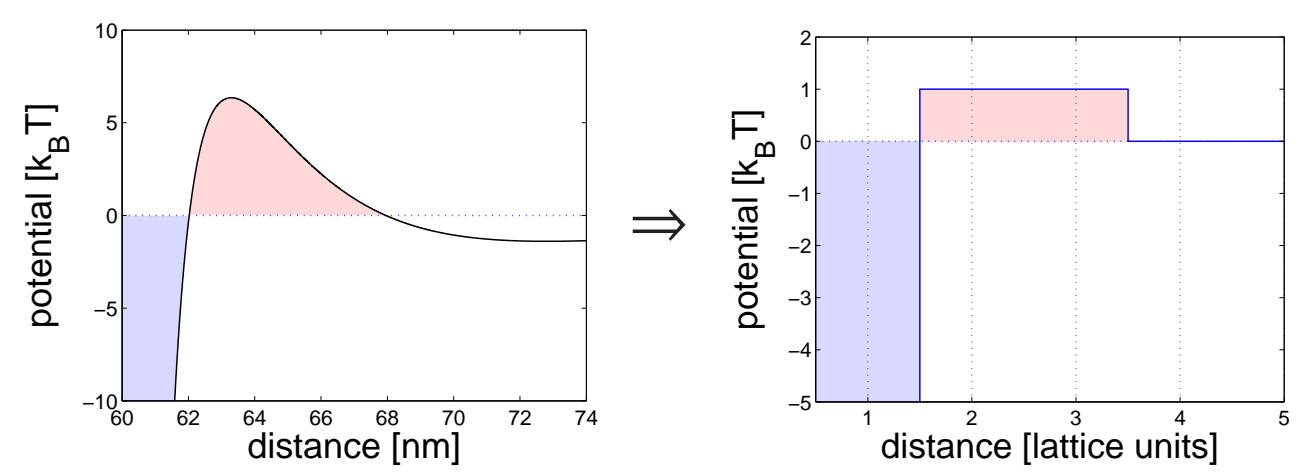

Figure 4.18 - Abstraction from the DLVO potential (left) to the simplified discrete potential used in the $\mathrm{MC}$ simulation (right). The short range attractive components (light blue) and repulsive barrier (bright red) are highlighted. Note that the axes are limited for clarity and crop the depth of the attractive potential well.

- Short-range attraction: the van der Waals/Casimir force is modelled as a potential well of constant depth $-39 k_{B} T$, ranging from 0 to 1.5 lattice units. This includes the four nearest neighbours, but also the four next-nearest neighbours, since $\sqrt{2}<1.5$. This deep well essentially traps adjacent colloids, hence it becomes extremely unlikely for a colloid to leave an aggregate.

- Repulsive barrier: the repulsive hump becomes a constant $1-10 k_{B} T$ spanning from 1.5 to 3.5 lattice units (felt at 28 lattice sites around a colloid). This models the DLVO potential in the regime close to the coagulation threshold.

Note also that the model potential effectively cuts off after 3.5 lattice units, i.e. there are no long-range interactions between colloids.

The potential landscape in the lattice is exemplified in Fig. 4.19, showing the potential of an aggregate and a single colloid. Plot $\mathbf{A}$ recalls the model potential of a single colloid, plot $\mathbf{B}$ shows how the additivity of potential fields increases the repulsive barrier, and the colour-map in plot $\mathrm{C}$ shows an excerpt $(0.64 \%)$ of the spatial potential landscape explored in the simulation. Note how the spatial additivity of the potential leads to 


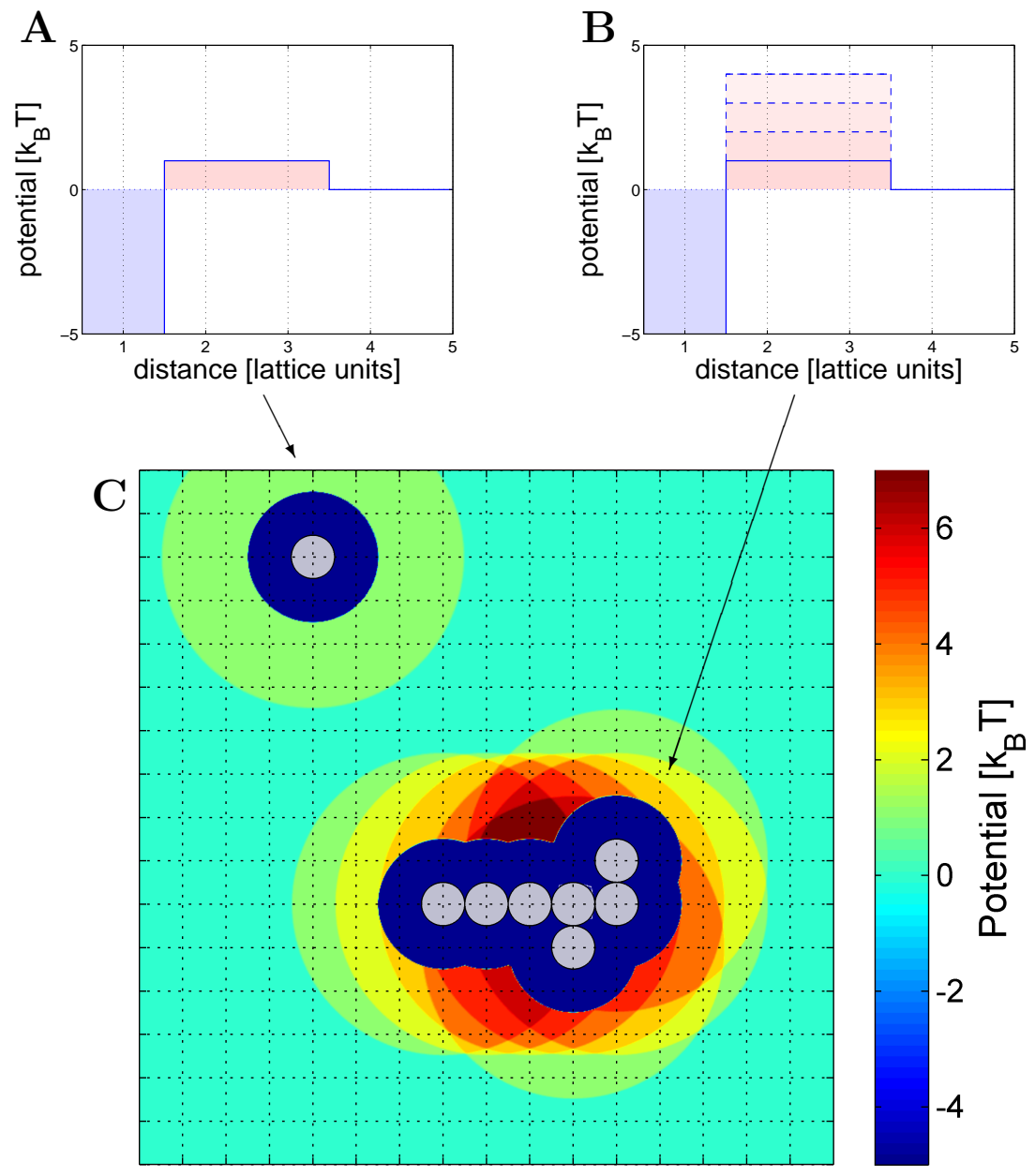

Figure 4.19 - Exemplary potential energy surface around a single colloid and around an aggregate for $V_{\text {barrier }}=k_{B} T$. Plot $\mathbf{A}$ shows the single colloid potential, plot $\mathbf{B}$ depicts the additive overlap of potentials leading to an effective increase in the Coulomb hump. Surface $\mathbf{C}$ colour-codes the potential landscape in the lattice resulting from a single colloid and a fictive aggregate of seven colloids. Note how the repulsion is weakest at the pinnacle ends of the cluster. 

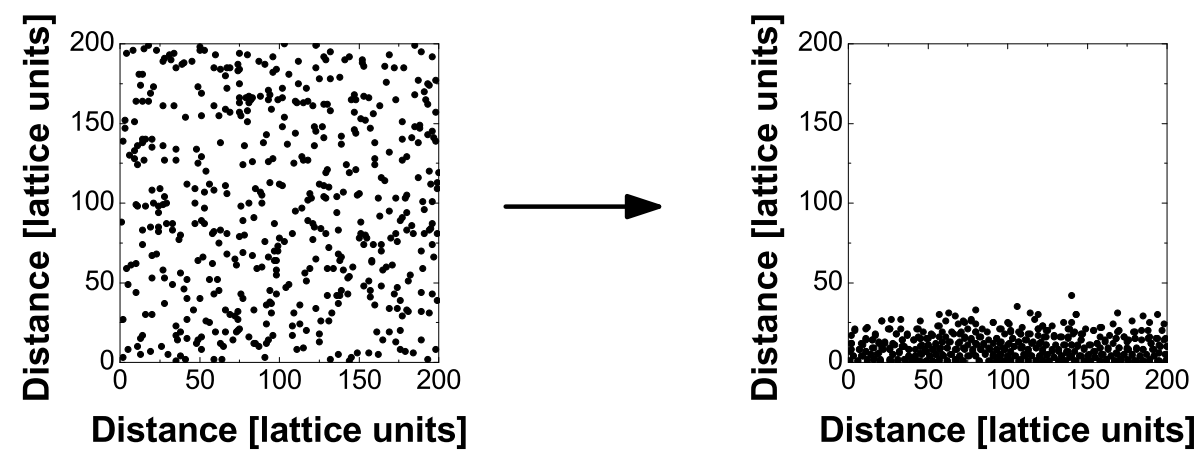

Figure 4.20 - Monte Carlo simulation of self-limiting aggregation (square lattice $200 \times 200$, 500 particles, closed boundaries, Metropolis algorithm, positive Coulomb barrier with $\left.V_{\max } / k_{B} T=10\right)$. In order to maximise the collision rate and interactions among clusters a small asymmetry $(1 \%)$ in the selection of the random movements in the Metropolis algorithm is introduced. After several million iterations this tends to accumulate the formed clusters towards a single boundary. The initial (left plot) and the final (right plot) configurations are shown. This forces the clusters to interact among themselves and collapse into bigger clusters if they find the chance.

direction-dependent adhesion probabilities for a colloid to join a cluster.

\section{Kinetic Monte Carlo simulation}

To provide an unbiased initial state, an ensemble of 500 colloids is randomly distributed over the lattice. Then, a Metropolis algorithm alters the lattice particle configuration through a large number of MC steps, whereby each iteration follows the algorithm:

1. pick a random colloid $\mathrm{c}_{i}$,

2. calculate its current potential energy $U$ by summing the potentials of all surrounding colloids, $U=\sum_{j \neq i} V\left(\mathrm{c}_{j}\right)$,

3. throw a random number to determine whether to move $c_{i}$ up, down, left or right by one lattice site, 
4. calculate the energy $U^{\prime}$ which $c_{i}$ would have in the new position,

5. if $U^{\prime} \leq U$, accept the move,

6. if $U^{\prime}>U$, accept the move with a Boltzmann probability $p=\exp \left(\frac{U-U^{\prime}}{k_{B} T}\right)$.

This scheme is looped until the desired number of iterations has been achieved. The method becomes kinetic by also accepting iteration steps that lead to a higher total energy, but only with a Boltzmann probability $\propto$ $\exp \left(-\Delta U / k_{B} T\right)$. By doing so Brownian motion is taken into account during the simulation and thus the approached final particle distribution is closer to physical reality. The picture however remains completely qualitative even for a 3D-lattice, for a number of reasons, e.g. the inaccurate potentials and lattice units that are coarse compared to the colloid size.

One method to assess the system's convergence after $N_{\text {iter }}$ iterations is to simply re-run the simulation for $2 N_{\text {iter }}$ iterations, and evaluate whether the desired properties (aggregational state) differ significantly, i.e. have not converged yet. In this context, a slight asymmetry is introduced in the Metropolis algorithm: In the arbitrary movement of a colloid, the down-direction is set to be $2 \%$ more likely to occur than the up-direction ${ }^{13}$, which fulfils two purposes: (i) simulate gravitational bias in the Brownian motion and (ii) thus ensure that colloids constantly interact with each other, since the effective 2D-volume is decreased (see Fig. 4.20). To that end, the simulation is biased towards exploring the effect of the additive potentials (i.e. Coulomb-blocking), since the number density of colloids/clusters, and thus the collision rate, is kept high.

\section{Results}

The results are summarised in Fig. 4.21: After $10^{6} \mathrm{MC}$-steps single colloids still dominate the picture; $16.6 \%$ of colloids have formed aggregates (31 dimers, 3 trimers). After $51 \cdot 10^{6} \mathrm{MC}$ iterations the predominant cluster

\footnotetext{
${ }^{13}$ The explicit probabilities are $26 \%$ up, $24 \%$ down, $25 \%$ left and $25 \%$ right.
} 
Figure 4.21 - Monte Carlo simulation of self-limiting aggregation (square lattice $200 \times 200$, 500 particles, closed boundaries, Metropolis algorithm, positive Coulomb barrier with $\left.V_{\max } / k_{B} T=10\right)$. Figures (a) to (d) show the histograms of the number of particles participating in clusters of size 1, 2, 3, etc., after $10^{6} \mathrm{MC}$-steps in (a), and then for an additional $5 \cdot 10^{7} \mathrm{MC}$ iterations in each step from (b) to (d). The predominance of dimers in the long term is a consequence of the particular choice of barrier height. The long term survival of small clusters in this conditions is a demonstration of Coulomb-limited aggregation.
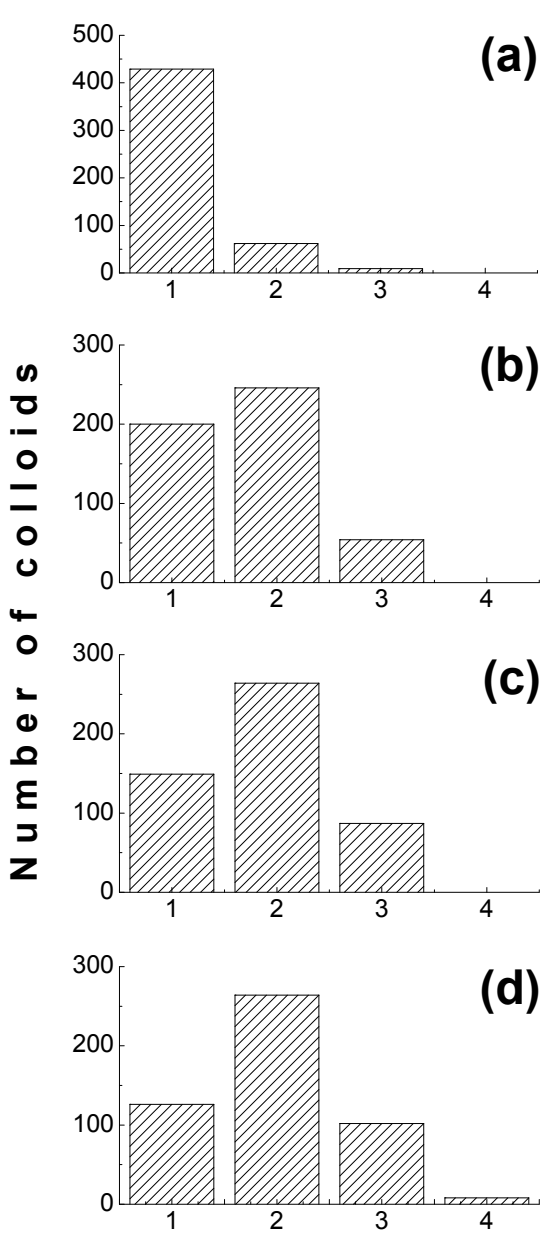

Cluster size [\# of colloids]

type is dimer ( $49 \%$ of all 500 colloids). Doubling the number of iterations to $101 \cdot 10^{6}$, dimers still dominate ( $53 \%$ ), and although trimers have gained $(17 \%)$, no tetramers have formed yet. After $151 \cdot 10^{6}$ iterations, the dimer population is left unchanged, the number of trimers grows only slightly $(22 \%)$, and the first two tetramers have appeared.

Evidently, aggregation continues throughout the entirety of the simulation, albeit at an ever-slowing rate. Nonetheless, the incessant dominance of small clusters (dimers, trimers) in the presence of many particles (with clusters being approached by other clusters/particles from all possible directions and with different energies) leads to a state in between the stable colloid and the fully aggregated situation. This state is metastable and con- 
tinues to evolve over time/MC steps. Metastability is, of course, a problem of definition of time-scales. A metastable colloidal state lasting for one year with an exponentially negligible probability of forming larger clusters becomes, for all practical purposes, a stable state. The dominance of clusters with a typical size (dimers in the case of Fig. 4.21) is a manifestation of self-limiting aggregation.

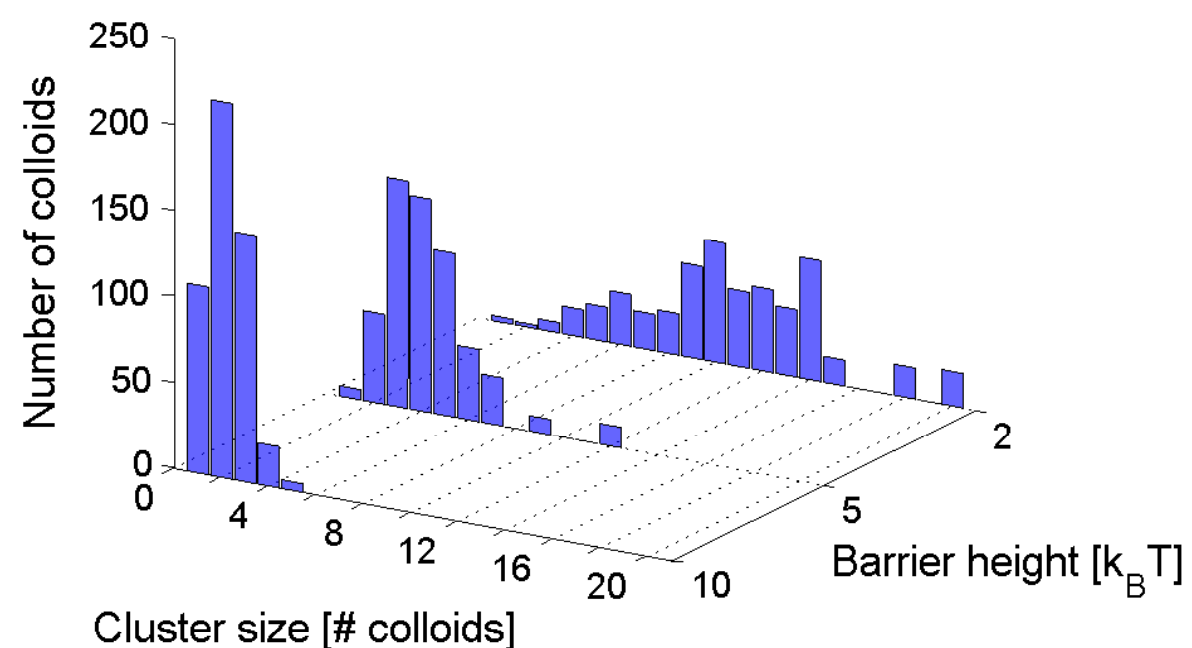

Figure 4.22 - Monte Carlo simulation of self-limiting aggregation (square lattice $200 \times 200$, 500 particles, closed boundaries, Metropolis algorithm, positive Coulomb barriers of 2, 5 and $10 k_{B} T$ ). The plot shows three histograms of the number of particles participating in clusters of size $1,2,3$, etc, after $2 \cdot 10^{8} \mathrm{MC}$ steps. Each histogram corresponds to a fixed potential - the barrier height is given on the $y$-scale. The distribution of cluster sizes broadens and shifts to larger values for shallower repulsive barrier heights.

The additive Coulomb barrier of the aggregated clusters makes it exponentially more difficult for further aggregation to occur. In fact, the dominant type of cluster in the final metastable state can be tuned by choosing different barrier heights or, alternatively, different temperatures for a fixed barrier height. For a slightly lower barrier, trimers become the dominant feature after several million MC-iterations, and so on. This correlation is clearly depicted in Figure 4.22 - here histograms of the number of colloids 
involved in different cluster types are shown for three different barrier heights. Each of the histograms corresponds to the resulting particle distribution after a $2 \cdot 10^{8}$-step MC simulation. Therefore the histogram shown for the $10 k_{B} T$ barrier is an extension of the series of histograms shown in Fig. 4.21(a-d). For shallower barriers the particle configuration noticeably shifts to larger clusters, i.e. mainly trimers, tetramers and pentamers for a $5 k_{B} T$ barrier and a broad distribution around 10-mers in the $2 k_{B} T$ case.

In a real situation, the colloidal solutions contains a variety of colloids with different sizes, shapes, surface charges, and therefore different interaction potentials. One would therefore expect a wider distribution of cluster sizes. Nonetheless Monte Carlo simulations are a powerful tool to find stationary solutions of many-body systems (in fact, theoretical physicists call MC simulations 'experiment'). To this end, the predicted state with self-limiting aggregation can explain both the high SERS activity of certain solutions and the long term stability by the same token. It is made plausible by the Monte Carlo simulation. 


\subsection{Conclusion}

With the theoretical interaction potential, the SEM-based characterisation, the clarifications regarding the effect of salt and the long-term stability of aggregated colloids, the thorough characterisation of the Lee \& Meisel colloidal system in SERS is, to this end, complete. The salt-induced SERSactivation is understood as an aggregation phenomenon, which provides, with the knowledge of the field redistribution in colloidal dimers, a consistent picture for the electromagnetic nature of the enhancement in SERS. The Coulomb-blocking mechanism retains the small-cluster state of aggregation in the colloidal system after SERS-activation, leading to SERS-active liquid samples with a long shelf-lifetime. 


\section{Chapter 5}

\section{Single-molecule SERS and Bi-Analyte SERS}

Human beings, who are almost unique in having the ability to learn from the experience of others, are also remarkable for their apparent disinclination to do so.

Douglas Adams

The schematic in Fig. 5.1 summarises and reviews the introductory part of this chapter. Before diving into the depths of Bi-Analyte SERS, I will outline the historic development of single-molecule (SM) SERS and show that its early proof -the Poisson distributed intensities- becomes untenable when viewed from today's perspective.

\subsection{Early SM-SERS claims}

In 1997, two papers by Nie \& Emory [9] and Kneipp et al. [8], renewed the interest in SERS by demonstrating single-molecule (SM) sensitivity of the technique. The impact of these works in the field of SERS is hard to 
overestimate - however, with the benefit of hindsight, some substantial uncertainties and flaws in these works surface after critical examination. Thus, it is the intention of this section to remove -without derogating impact- some ultimately false claims and to clear up the picture these early studies presented.

\subsubsection{The ultra-low concentration approach}

The initial claims of SM sensitivity were based on a probabilistic approach. The basic idea is to use ultra-low analyte concentrations in colloidal solutions, to the point where it becomes unlikely that more than one molecule attaches to a single colloid ${ }^{1}$, and then to try to observe signal from single colloids/colloidal clusters. This approach poses some serious experimental difficulties, most importantly:

(i) the dilution to ultra-low concentrations is error-prone (as pointed out by Hildebrandt [109]), since normally overlooked effects become important (e.g. wall adsorption).

(ii) the estimation of the number density of colloids suffers from uncertainties. Specifically, the estimates are based on the mass of silver used in the synthesis. This results in inaccurate numbers due to the typically strong nucleation of Ag on the container walls, which does not contribute to the colloidal ensemble. Also, the exact colloid size and shape distributions are unknown.

Furthermore, the logic of this approach becomes questionable in view of today's knowledge about the source of the highest enhancements and signals: the SERS hot-spot. SM detection requires high-enhancement hotspots which typically arise from the aggregation of colloids, the canonical

\footnotetext{
${ }^{1}$ Kneipp et al. added crystal violet to a final concentration of $3.3 \times 10^{-14} \mathrm{M}$ in the wet sample and estimated an average of 0.6 molecules per colloid; Nie et al. prepared dry samples and used Rhodamine $6 \mathrm{G}$ at concentrations of $2 \times 10^{-10} \mathrm{M}$ and $2 \times 10^{-11} \mathrm{M}$, estimating a surface number density of 1 and 0.1 respectively.
} 


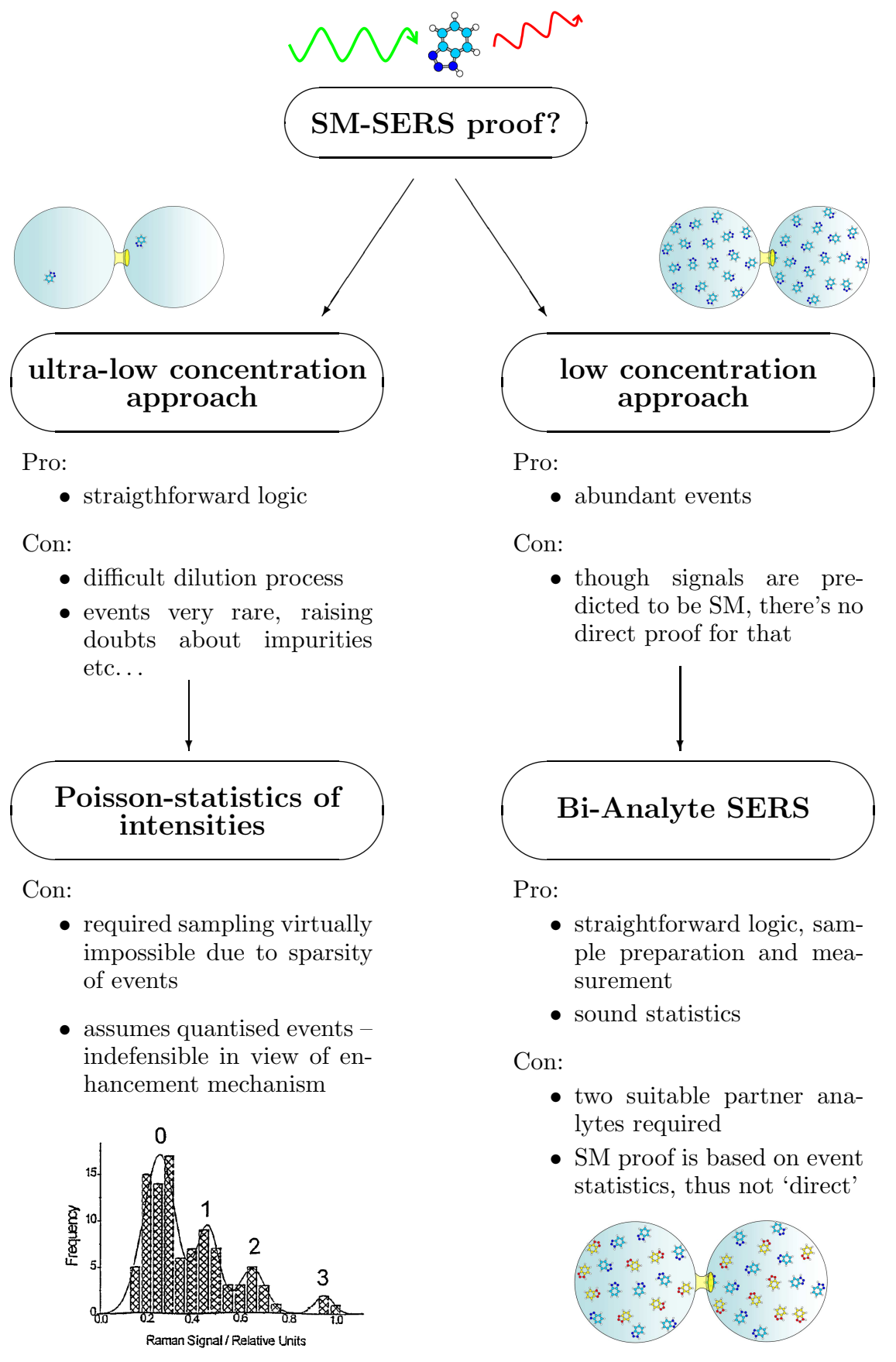

Figure 5.1 - Schematic overview of the SM-SERS topic. Each SM-proof approach and their stated characteristics are explicitly adressed in the text below. 
example being the colloidal dimer (see Sec. 3.1.5). Such hot-spots are locally confined on the colloid's surface, with most of the intensity coming from a tiny region: GMT calculations for a typical dimer show (see Ref. [66]) that $80 \%$ of the signal originates from a $9^{\circ}$ cone around the dimer axis, corresponding to $0.66 \%$ of each colloid's surface (see Sec. 3.1.5). If one was to prepare a solution with an average of 0.6 molecules per colloid, the probability that a molecule comes to lie within that $80 \%$-signal cone would be $0.0066 \approx 1 / 150$. Therefore, even in the case of each hot-spot being perfectly aligned in the incoming field, the average chance to see a single molecule is $0.6 \times 0.0066=0.4 \%$, i.e. one discernable event per $\sim 250$ observations. Thus comprehensible, Nie \& Emory [9] assert that "only one out of perhaps 100 to 1000 particles is hot" and "only one out of 10000 surface sites on a hot particle shows efficient enhancement".

It is thus reasonable to conclude that in the ultra-low concentration regime the chance to observe single molecules is minimal. A proof based on rare events will, though logically correct, always face doubt about the authenticity of events - simply put, the apparent SM events could well be 'outliers' caused by impurities or other experimental uncertainties. For example, the rod-like colloidal aggregated mentioned earlier (see Sec. 4.3.4) will absorb more dyes than isolated colloids, and can thus produce rare, high-intensity events. The intrinsic sparsity of events is therefore a doubleedged sword: on the one hand validating the claim, on the other hand fuelling its dispute.

Having gained these insights, it would now seem natural to stop venturing further and instead explore a more suited approach. Before doing so, however, a notable chapter in the history of SM-SERS requires special attention. 


\subsubsection{Poisson distribution - quantised intensities?}

The rebuttal of Poisson-statistics in SM-SERS was done in full in Ref. [75], however, a brief review of the key points shall be given here.

While Nie \& Emory's paper [9] used ultra-low concentrations and took the fact that Raman signatures are still observable ${ }^{2}$ as proof for SM sensitivity, Kneipp's paper [8] goes one step further and analyses the statistics of the intensities of apparent SM-SERS events. The analysis is based on 100 spectra, whose intensities are binned and plotted in a histogram (see Fig. 5.2). The histogram shows a structure of peaks, whereas peaks at higher intensity exhibit less observation probability. This is attributed to events in which $0,1,2$ or 3 molecules partake, with zero molecules being the most probable case. Four Gaussian curves are fitted to the $\sim 20$ data points of the histogram. The paper states that the areas of these Gaussians are "roughly consistent with a Poisson distribution for an average number of 0.5 molecule." This is in good agreement with the estimated number density of 0.6 molecules per colloid, however, this in turn implies a 80\% SM detection efficiency - a direct contradiction to the rarity of events observed by Nie \& Emory.

Accordingly, one is left with a conundrum: How could one possibly observe sufficient spectra to distinguish these ultra-rare events (all on the edge of detectability) from sample impurities, let alone derive meaningful statistics from it?

Nonetheless, at first sight, the observation of signals from ultra-low concentration samples and the seeming logical consistency of the Poissondistributed intensities serve as powerful arguments for the single-molecule sensitivity in SERS. However, with a bit of further thought a series of questions arise, in particular pertaining to the Poisson-fit, to wit:

- Why is there a peak for the zero-molecule case, and why is it not at zero intensity? How exactly were spectral intensities evaluated?

\footnotetext{
${ }^{2}$ The rarity of events (see Sec. 5.1.1) being the only source of doubt (possible impurities).
} 


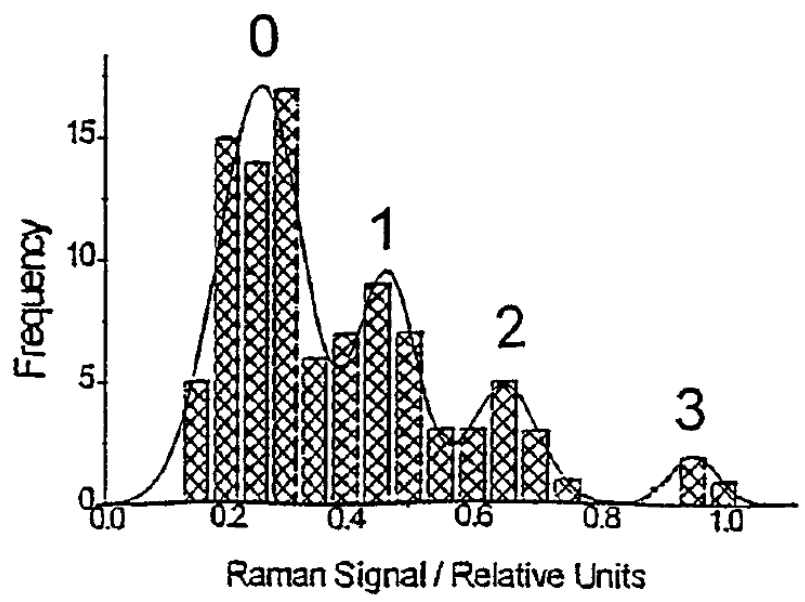

Figure 5.2 - Reproducted from Ref. [8] with permission (copyright: American Physical Society). Histogram of intensities for 100 events recorded from a Lee\&Meisel Ag colloidal sol with a $33 \mathrm{fM}$ concentration of crystal violet. The apparent structure is attributed to the number of molecules visible per event. See text for details.

- Why are the peak positions not equidistant? This directly contradicts the underlying assumption of quantised intensities.

- Why is the width of peaks not monotonically increasing for higher intensities, but rather varying randomly? This has a large impact on the peak areas, which are said to correlate Poisson-like.

Above all, what is the significance of such an elaborate statistical analysis if based on only 100 values? With the present understanding of the SERS enhancement mechanism, it is easy to convince oneself that apparent structures (i.e. peaks) in histograms of SM-SERS intensities are an artifact of insufficient sampling. The weakest point of the Poisson-like intensity distribution is the underlying assumption of quantised intensities. SERS intensities have to be quantised to at least within a factor of $\sim 2$, if Poisson-statistics were to be observed [75]. If the intensity of a molecule varies by more then a factor of 2 , it becomes impossible to distinguish the intensity of a single molecule from that of two molecules, and so forth, thus invalidating the applicability of a discrete probability distribution. 


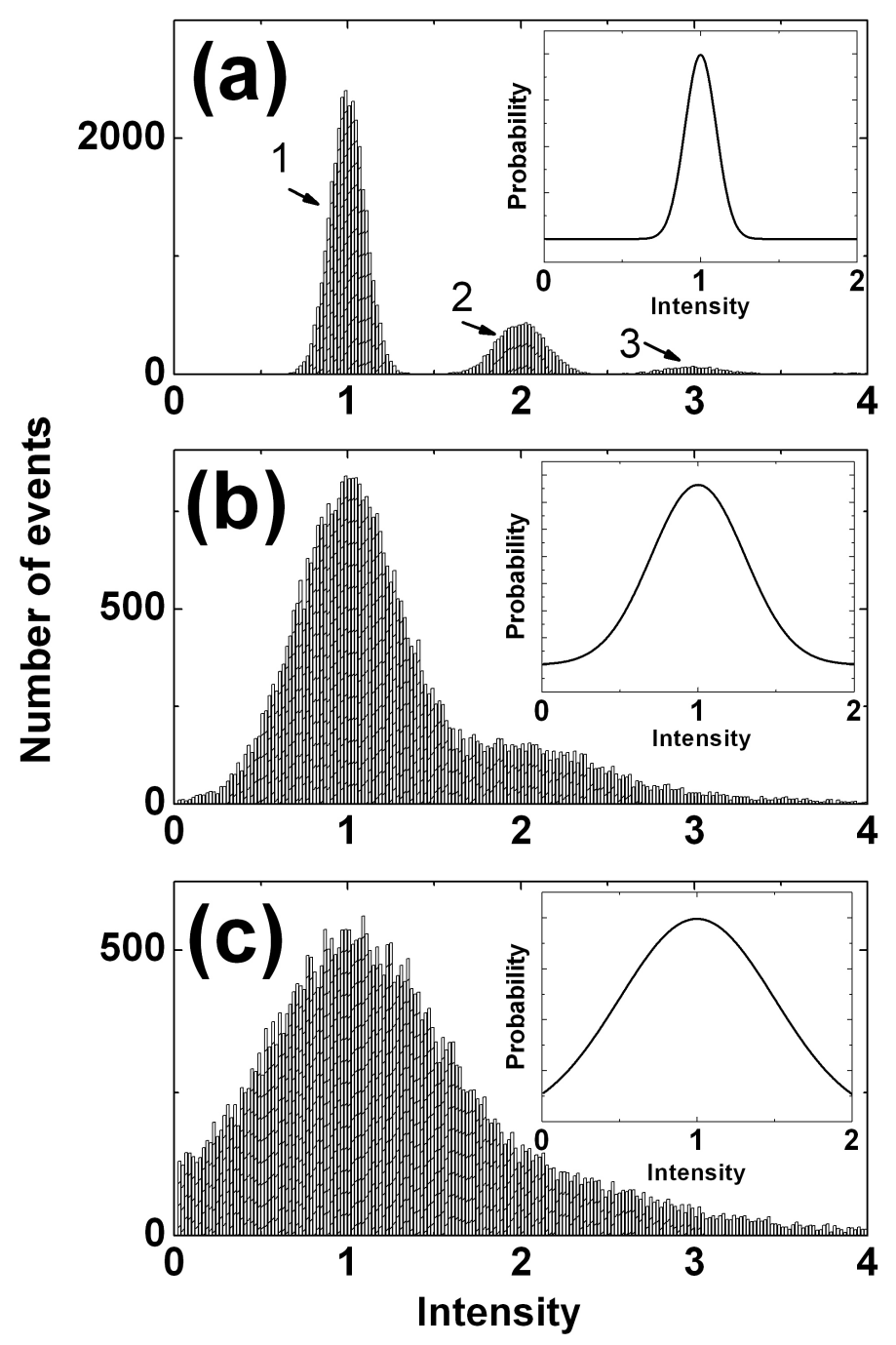

Figure 5.3- Histograms of number of cases with a given intensity for three different scenarios of intensity distributions. In all examples, the number of molecules $\left(10^{5}\right)$ follows a Poisson distribution (imposed) with $\mu=0.5$, but each molecule contributes with an intensity taken from a Gaussian probability distribution (see insets) centered at $I=1$, with variance $\sigma=0.1$ in (a), 0.3 in (b), and 0.5 in (c). The peaks representing 1,2 , and 3 molecules can be clearly seen in the histogram. Note that as soon as the intensity distribution of individual molecules is spread over a region spanning a factor of roughly $\sim 2$ the ability to count the molecules from the intensity and the Poisson statistics picture is completely lost. A Gaussian distribution with a spread within a factor of two among single molecule events in hot-spots is virtually impossible to achieve in real SERS substrates of any kind. 
Basically, the Poisson-distribution of intensities is washed out (see Fig. 5.3) as soon as the unphysical assumption of highly confined SM intensities is dropped. Why is this assumption unphysical? There are many effects which counteract intensity quantisation, in particular in a disordered SERS sample:

- Observable SERS signals originate from hotspots. The enhancement a hotspot provides depends strongly on a range of parameters (colloid shape, cluster geometry, incident polarisation and relative orientation to it, etc...). Therefore, even when assuming that in each hotspot a single molecule were to be always perfectly centered, the hotspotto-hotspot variance in enhancement will still cause SM-SERS event intensities to vary by orders of magnitude.

- The Raman intensity emitted by a molecule depends strongly on its position within the hotspot; the stronger the enhancement, the stronger its spatial dependence. Typically, amidst regions which provide field enhancements sufficient to observe SM-SERS from, the enhancement factor varies by orders of magnitudes over a few nanometres (see Sec. 3.1.5 and Ref. [66].)

- Since the enhanced field vectors are preferentially normal to the metallic surface, the Raman cross-section of a molecule on the surface depends on its relative orientation thereon (see Sec. 6.8 and Ref. [69]).

- Last but not least: photo-chemical effects (e.g. photo-dissociation and photo-bleaching) aggravate the higher the photon density, and may thus significantly decrease the lifetime of single molecules under high enhancements. This in turn stochastically affects the emitted SM intensity. ${ }^{3}$

\footnotetext{
${ }^{3}$ Photo-chemistry introduces a natural boundary for a hotspot's field enhancement for observable SM-SERS events: while the emitted SM intensity increases with field enhancement, the molecular lifetime decreases. If the local field is too intense, one may never observe the molecule since it 'dies' (due to fast bleaching) before enough scattered photons to discern it from noise can be collected.
} 
Therefore, it is safe to conclude that it is not possible to count molecules from their emitted SERS intensity, and hence the foundation for a discrete probability distribution is nonexistant. Correspondingly, one can conclude that the proof of SM-sensitivity in SERS delivered by Nie \& Emory and Kneipp was thus at best incomplete; it was recognised as such by the leading authors in their joint paper "Single-molecule Raman spectroscopy: fact or fiction?" (Ref. [12]). Nonetheless, the Poisson-statistics based proof spurred interest and development in SERS (and is accordingly widely cited), and -for lack of a better proof- remained unchallenged in the SERS community. Furthermore, the proof was repeated by other groups, for example in Ref. [110] (a paper so similar to Ref. [8] that it may be confused with the original) and Ref. [111], which applies the same logic to tip-enhanced Raman spectroscopy (TERS) ${ }^{4}$. The proof was re-emphasised numerous times by the Kneipp group (for example Refs. [112, 79, 81]). Eventually, a decade after its initial conception, the validity of the Poisson-argument was refuted [14, 75, 113, 21].

\subsection{Recent SM-SERS efforts}

Subsequent to the discovery of SM-SERS in 1997, many groups applied the ultra-low concentration approach (for example, see Refs. [31, 114, 115, 65, $116,117,118])$. The newly found results were put on a theoretical footing by a number of publications which addressed the mechanisms behind the surprisingly large enhancements (see for example Refs. [119, 120, 121, 83, $122,123,124])$. This accumulated a 'body of evidence' for SM-SERS, which was -however- eroded by the intrinsic shortcomings of the initial proof (see above).

Conversely, a number of recent papers ventured from the conventional SM-SERS scheme (i.e. ultra-low concentration of dye in Lee \& Meisel Ag

\footnotetext{
${ }^{4}$ Under TERS conditions, many of the criticisms against the original proof disappear, however, quantised intensities remain hard to justify.
} 
colloids) and plausibly demonstrated SM-SERS, thus adding significantly to its credibility. These deserve a mention here:

- The works by Aroca et al. $[125,19,126]$ created a notable advance in SM-SERS by increasing the control on the experimental SERS system through Langmuir-Blodgett films. In the developed technique, a SERS sample is created by deposition of a dye-containing fatty-acid Langmuir-Blodgett film onto a silver-island substrate. The density of analytes within the film is well controllable, which specifically curbs one of the salient uncertainties in the previous claims - the number density of analytes on the substrate. The technique was also successfully combined with the Bi-Analyte approach (see next section), see Ref. [127].

- Tip-enhanced Raman spectroscopy (TERS) is a recently developed technique [128] which combines the surface-enhancement effect with scanning-probe microscopies (e.g. AFM, SNOM, STM) [129, 130, 131]. By scanning the laser-illuminated probe tip it becomes possible to selectively provide enhancement to a small area on a substrate (beyond the diffraction limit), which, assuming a sufficiently low analyte surface density, should correspond to single molecules. Promising results towards SM detection were achieved by the groups of Raschke [111] and Pettinger [132], albeit the former being subject to criticism $[133,134]$ regarding the Poisson-based proof (see above).

- Maher et al. [135] observed photobleaching under SERS conditions and noted, for low analyte concentrations, that the decay of the intensity happens in a stepwise fashion. This added to the plausibility of a situation in which only a few individual molecules contribute to the observed signal.

While all these methods have their strengths and weaknesses, and can be pursued as valid routes for single molecule SERS detection, I will (naturally) concentrate on the method that was developed in our laboratory 

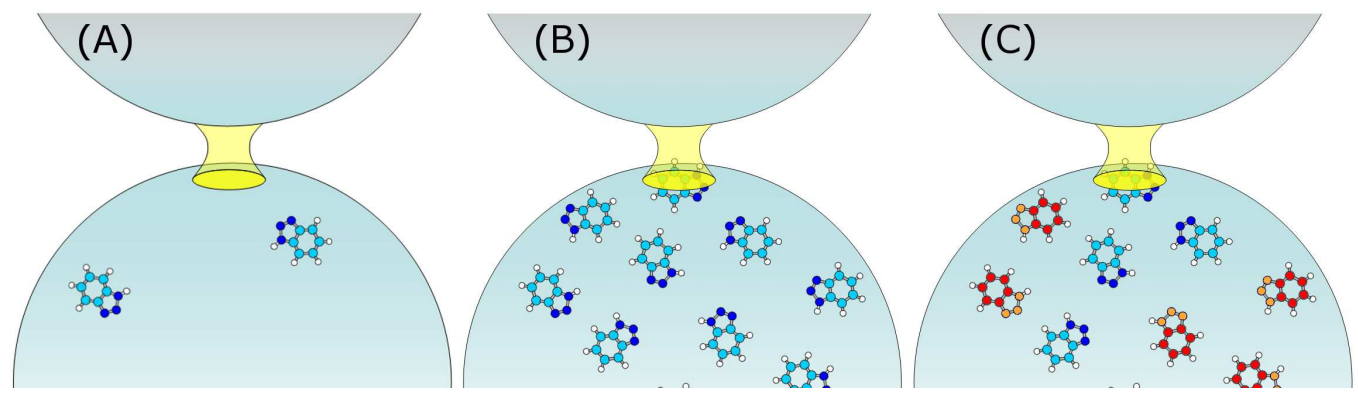

Figure 5.4 - Cartoon of SM-SERS scenarios: (A) depicting the ultra-low concentration regime, in which the analyte surface density is so low that it becomes unlikely that a molecule resides at the hotspot - events are very sparse. (B) High analyte surface density, it is likely that molecules are in the high-enhancement region. Events are abundant, but since the 'extent of the hotspot' is unknown, one cannot prove or disprove SM-SERS from here. (C) Introducing the contrast method Bi-Analyte SERS: retaining abundant signals, one can prove SM-SERS with simple arguments and use the technique to identify SM events. See text for details.

during this thesis; a method based on the use of two analytes as "contrast" to statistically detect the occurrence of SM events. Hence, in the next section and thereafter, I will concentrate only on this method.

\subsection{Rigorous proof of SM-SERS using two ana- lytes}

\subsubsection{The Bi-Analyte SERS idea}

The Bi-Analyte SERS (BiASERS) idea was born out of mishap - an accidental discovery. Nonetheless, the idea is so simple that, in retrospective, it borders on embarrassment contemplating that one was unable to come up with it any earlier ${ }^{5}$. The localisation of the enhancement in SERS sam-

\footnotetext{
${ }^{5}$ In fact, Zhang et al. [136] missed this opportunity to settle the SM-SERS discussion by a remarkably close margin.
} 
ples leads to rare events if only few molecules populate the substrate (see Fig. 5.4). Increasing the analyte concentration leads to more abundant events, however, with more than one molecule per colloid, the only argument for SM detection is gone. This can be restored by using two analytes at the same time - if the observed signal comes from many molecules, one expects to see a spectrum composed of both analytes' spectra; however, if a single or few molecules contribute, then one expects to see pure spectra, i.e. one analyte or the other. Conversely, pure spectra are extremely unlikely to happen if many molecules contribute, hence, observing pure spectra is a proof of the SM-nature in SERS signals.

\subsubsection{Discrete model hot-spots}

Assume a SERS active sample with two analytes A and B, for which in each SERS event the observed signal originates from exactly $N$ molecules. This implicitly assumes that hot-spots are 'bucket-like', i.e. have a sharp boundary, and molecules are either within the hot-spot or outside of it. Moreover it assumes all hot-spots to be of a very limited spatial extent, so that each hot-spot is populated by only (and exactly) $N$ molecules. Let us furthermore assume that we are obtaining a series of spectra from this model sample, with each measurement being that of an independent, random selection of $N=N_{\mathrm{A}}+N_{\mathrm{B}}$ molecules from the pool of the two analytes. Then, in the general case, spectra will be of a mixed type (i.e. both dyes $\mathrm{A}$ and $\mathrm{B}$ present); however, there is a chance for pure spectra to occur (i.e. $N_{\mathrm{A}}=0$ or $N_{\mathrm{B}}=0$ ). Figure 5.5 exemplifies this model for the case $N=3$.

With this model in mind, we can now ask: For any SERS event, what is the probability $p_{A}$ of all $N$ molecules being of type A? If $N=1$, then every event will either be from analyte $\mathrm{A}$ or $\mathrm{B}$, thus $p_{A}=1 / 2$. If $N=2, p_{A}$ drops to $1 / 4$. For $N=3$, we get $p_{A}=1 / 8$, etc... More generally, the probability $p_{A}$ 

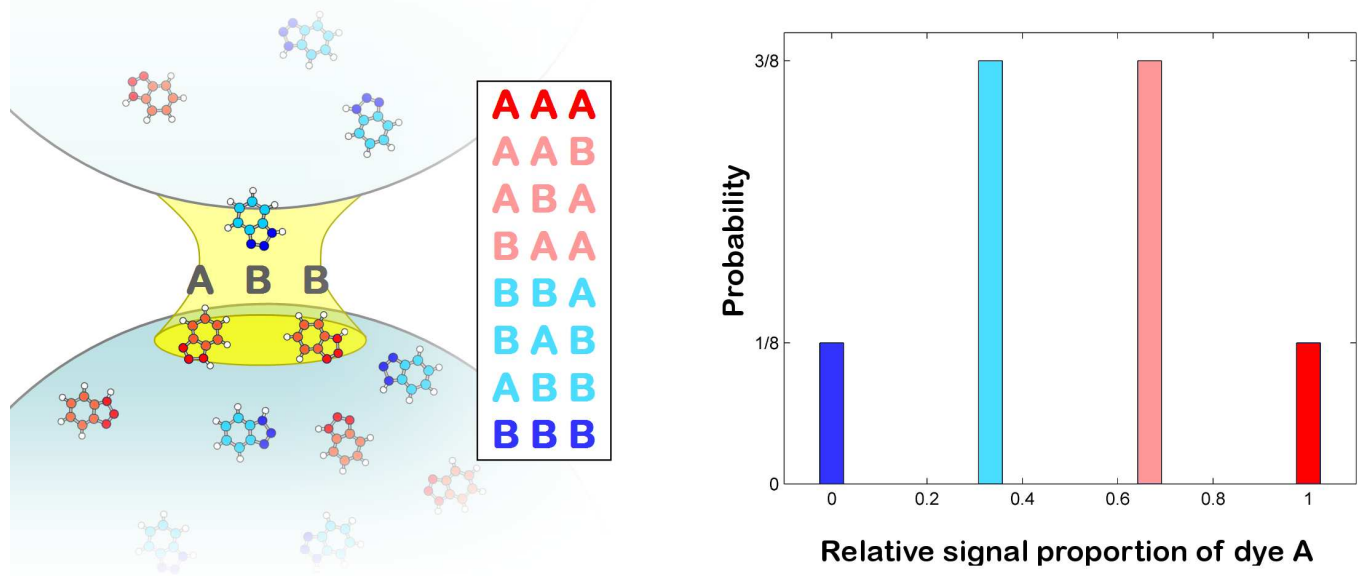

Figure $5.5-$ Left: Cartoon exemplifying the case $N=3$. The hot-spot fits 3 molecules which are selected at random with equal probability. Shown are 8 possible combinations of dyes $A$ and $B$, two of which are pure (red, blue), three are $2 / 3$ dye $A$ plus $1 / 3$ dye $B$ (pink) and the remaining three are $1 / 3$ dye $A$ plus 2/3 dye $B$ (light blue). Right: Trivial histogram showing the discrete probabilities of occurrence for this case. See also Fig. 5.7.

to observe a pure signal from dye $\mathrm{A}$ (or dye $\mathrm{B}, p_{B}$ ) is:

$$
p_{A}(N)=\frac{1}{2^{N}} \quad, \quad p_{B}(N)=\frac{1}{2^{N}}
$$

The fraction of expected pure spectra $(\mathrm{A}$ or $\mathrm{B})$ then is:

$$
p(N)=p_{A}(N)+p_{B}(N)=\frac{2}{2^{N}}
$$

Accordingly, the probability to observe a pure spectrum drops rapidly: for $N=10$, we get a chance of less than 1 in 1000 to observe a pure spectrum from either dye A or B. Figure 5.6 plots the probability to observe pure (and mixed) signals as a function of small $N$.

It is the rapid decay of $p$ with increasing $N$ which constitutes the SM proof, by falsification: if each SERS signal originates from many molecules $(N \gg 1)$, then the probability to observe a pure spectrum is extremely small $(p \rightarrow 0)$, and such an event becomes highly unlikely. Thus, in a SERS 


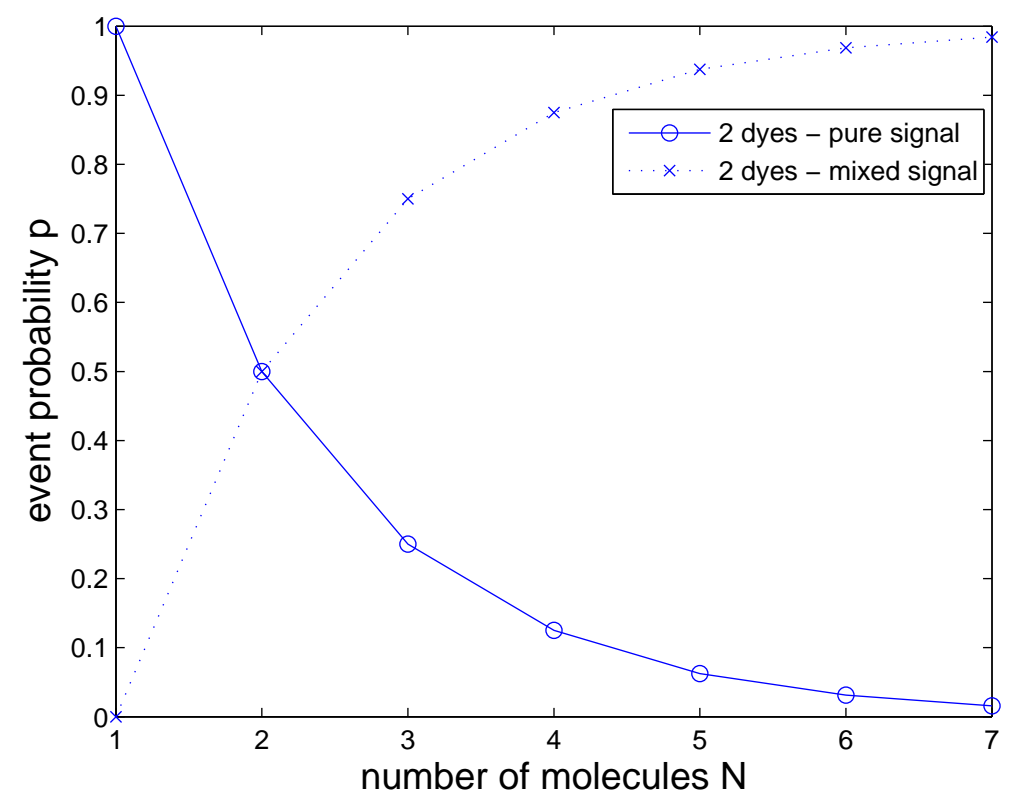

Figure 5.6 - Event probabilities for the bi-analyte model scenario. Shown is the probability of observing pure signals (solid lines, circles) and mixed signals (dotted lines, crosses) as a function of the number of molecules $N$ participating in each event.

measurement where pure spectra occur regularly (evidently $p>\epsilon$, with $\epsilon$ being some reasonably high threshold $\left.{ }^{6}\right)$, these signals must stem from single or few molecules $(N \sim 1)$.

\section{Probability and spectral compositions}

Within the model of discrete hot-spots, for a fictive ensemble of spectra, one can trivially assess the participation each analyte has in each spectrum:

$$
P_{\mathrm{A}}=\frac{N_{\mathrm{A}}}{N} \quad \text { and } \quad P_{\mathrm{B}}=\frac{N_{\mathrm{B}}}{N}=\frac{N-N_{\mathrm{A}}}{N}=1-P_{\mathrm{A}}
$$

Once the participations are known for all spectra, the probability distribution thereof is determined. In the discrete hotspot model the probability

\footnotetext{
${ }^{6} \epsilon$ should be well above $1 / 1000$ to improve on the statistical significance of the ultra-low concentration approach.
} 


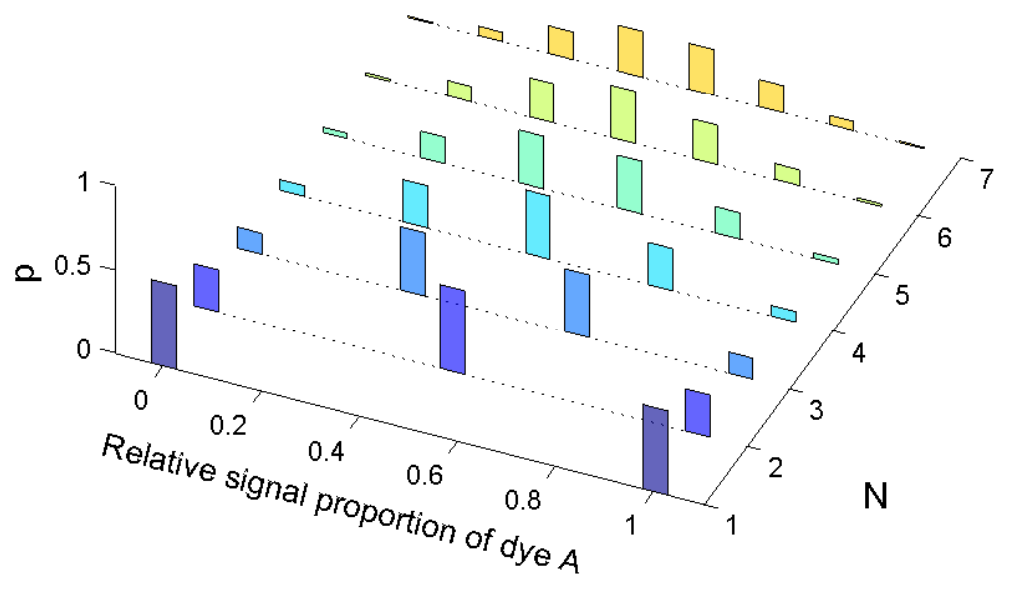

Figure 5.7 - Event probabilities for the bi-analyte model scenario for various small $N$ (number of molecules per hot-spot). The discrete probabilities for spectra having a relative percentage of dye $\mathrm{A}, P_{A}$, are shown. Accordingly, $P_{A}=1$ indicates a pure dye $\mathrm{A}$ spectrum, $P_{A}=0$ a pure dye $\mathrm{B}$ spectrum and $0<P_{A}<1 \mathrm{a}$ mixed spectrum. See text for more details.

distribution follows trivially (along the lines of Eq. 5.2) without formally requiring to assess spectral participations for each spectrum. In the experimental context however, this step cannot be omitted since the real-world notion of a SERS hotspot corresponds to a skewed enhancement distribution (see Sec. 3.1.5), and is not a discrete 'bucket'.

Hence, the analysis of real BiASERS data is a two-step process:

1. The spectral composition (participations $P_{\mathrm{A}}^{i}$ and $P_{\mathrm{B}}^{i}$ ) of each spectrum $i$ is evaluated. Note that, obviously, Eq. 5.3 stems from a concept model and has no relation to real data. Within more realistic SERS models the spectral percentage (and thus event-probability) will need to be revisited. Hence, these quantities will be explicitly redefined later in the text to suit our needs (see Sec. 5.3.4, Eq. 5.6).

2. The histogram of spectral percentages (i.e. its probability distribution) is analysed to answer the questions of the type: Do pure events occur? How likely is a pure or mixed event? On average, how many molecules contribute significantly to the signal? 


\subsubsection{Prerequisite criteria}

What are the basic criteria necessary for a BiASERS experiment?

- Clearly, both analytes, separately, must show SERS signals under the particular experimental system chosen.

- When mixed together, these analytes must not influence each other, e.g. analyte A must not prevent analyte B from surface adhesion.

- The spectra of both analytes must be distinguishable. This fairly obvious requirement may seem straightforward to fulfill, given that the Raman spectrum is often viewed as a molecular 'fingerprint'. However, the BiASERS technique benefits from the chemical and physical similarity of analytes (see below), and the criterion of spectral difference thus becomes non-trivial.

- The cross-section of both analytes must be comparable. If the ratio of cross-sections is too large/small, then measurements will yield little spectral contrast, with one spectral component 'buried' within the other. This will increase uncertainties about spectral percentages and eventually render any statistical analysis impossible.

- A comparable percentage of both dyes must adhere to the substrate's surface. Otherwise one faces the same problem of diminishing spectral contrast as in the previous criterion.

To summarise, the BiASERS technique requires analytes which have similar surface chemistries and spectroscopic properties, while retaining sufficiently different spectra - in other words, there is an uncertainty relation between molecular similarity and spectral contrast.

I will now move on to the first BiASERS experiment and evaluate the above criteria, as well as analyse it in terms of the discrete hotspot model. 


\subsubsection{BiASERS - experiment}

The first BiASERS experiment conducted and interpreted as such uses the two dyes Rhodamine 6G (Rh6G) and the benzotriazole dye BTZ2 (refer to Sec. 2.3 for an introduction to these species).

\section{Samples \& experimental details}

Citrate-reduced Ag colloid solutions were prepared as described earlier (see Sec. 4.1.2), Rh6G and BTZ2 (see Sec. 2.3) was added as analytes (to a final concentration of $100 \mathrm{nM}$ each) and colloidal aggregation was induced by diluting the solution by a factor of 2 using a $20 \mathrm{mM} \mathrm{KCl}$ solution (thus yielding a final concentration of $c_{\mathrm{KCl}}=10 \mathrm{mM}$.) Following this general recipe, three solutions were prepared:

Sol A: Aggregated colloids with 100 nM BTZ2,

Sol B: Aggregated colloids with $100 \mathrm{nM}$ Rh6G and

Sol C: Aggregated colloids with both, 100 nM BTZ2 and 100 nM Rh6G.

To highlight the contrast these samples represent with respect to previous ultra-low concentration measurements, the average number of molecules per colloid in sol C is, approximately:

$$
N_{\text {mol }}=\frac{c \mathcal{N}_{A}}{n_{\text {coll }}} \approx 1200,
$$

where $c$ is the overall dye concentration, $\mathcal{N}_{A}$ is Avogadro's number and $n_{\text {coll }}$ is the estimated number density of colloids $\left(\sim 10^{11} \mathrm{~cm}^{-3}\right)$. Assuming the average colloid's radius $R$ to be $30 \mathrm{~nm}$, one can estimate the surface area of an average colloid:

$$
A_{\text {coll }}=4 \pi R^{2} \approx 1.1 \cdot 10^{4} \mathrm{~nm}^{2},
$$

which translates into $\sim 9 \mathrm{~nm}^{2}$ per molecule - plenty of space for molecules with a maximum footprint of roughly $1 \mathrm{~nm}^{2}$ (see Appendix A). 


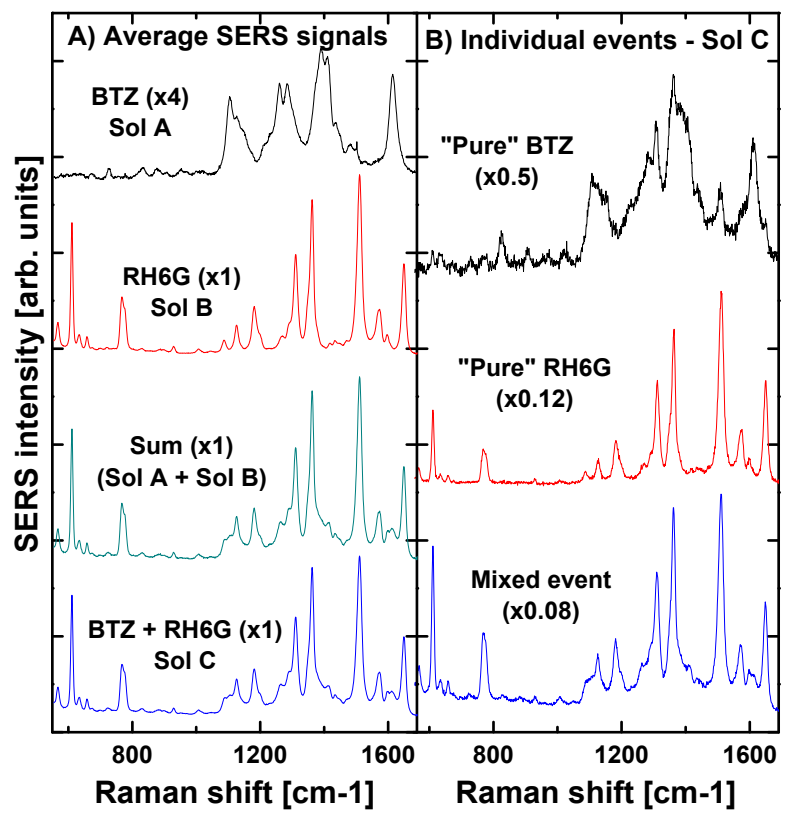

Figure 5.8 - Left: Average SERS spectra from solutions A, B and C. Also shown is the sum of spectra from $A$ and $B$, which is identical to the spectrum of solution $C$ within experimental errors. Right: Representative individual spectra (integration time $0.2 \mathrm{~s})$ of solution $\mathrm{C}$ showing a pure BTZ2 event $\left(P_{B}=0.91\right)$, a pure Rh6G event $\left(P_{B}=0.07\right)$, and a mixed event $\left(P_{B}=0.5\right)$. The arbitrary scale is the same on both sides. The pure BTZ2 event still shows very small peaks from Rh6G due to its larger cross section.

A $\times 100$ index-matched (to water) immersion objective was used; the excitation wavelength was $633 \mathrm{~nm}$, at $2 \mathrm{~mW}$ laser power. For all solutions average SERS spectra were collected. For solution C, also a time-series of 1000 spectra with an integration time of $0.2 \mathrm{~s}$ each was collected.

\section{Results}

The plot on the left side of Figure 5.8 shows (from top to bottom) the average spectrum of sol A, sol B, the sum spectrum of sol A and B, and the average spectrum of sol $\mathrm{C}$. From here, a number of conclusions can be drawn: 
- As expected, both dyes show strong Raman spectra. The spectra differ in many features, thus one can readily differentiate between signals from either of the dyes, and identify mixtures thereof.

- Rh6G's cross section is roughly four times larger than that of BTZ2 under these conditions. Though this is not ideal, the resulting spectral contrast is still large enough to clearly identify (and quantify) pure and mixed signals.

- The sum of the average spectra of sols A and B is virtually identical to the average spectrum of sol $\mathrm{C}$. This indicates that the two analytes in sol C do not interact with each other, and adsorb onto the colloid's surface independently.

Consequently, the prerequisites laid out in Sec. 5.3.3 are fulfilled.

The plot on the right side of Fig. 5.8 shows three exemplary spectra from the time-series of spectra from sol C. The most striking fact is the presence of pure spectra. Considering that there are $\sim 600$ molecules of each analyte adsorbed to every colloid, one might expect to only see mixed spectra. However, granted the background knowledge of hotspot localisation, these results become understandable: Pure spectra, arising from single/few molecules, indicate the strong localisation of the enhancement region on the colloidal surface. An estimate of the extent of this region will be eluciated further below.

\section{Evaluating spectral participations}

To evalute the statistics of the events, in particular the distribution of spectral participations, one needs to define how the spectral dependence is to be deduced from each spectrum. For this purpose, here, the reference spectra $I_{\text {Rh6G }}(\lambda)$ of Rh6G and $I_{\text {BTZ2 }}(\lambda)$ of BTZ2 are utilised. These are obtained separately (from sol A and sol B). Since the dyes in sol C adhere and scatter independent of each other, it suggests itself to express each event in the 
time-series as a linear superposition of the reference spectra, plus a function $B G$ allowing for changes in the signal background:

$$
I^{\text {theo }}(\lambda)=\alpha I_{\text {Rh6G }}(\lambda)+\beta I_{\mathrm{BTZ2}}(\lambda)+B G \quad,
$$

where $B G$ may, in general, depend on $\lambda$ and a set of parameters. In fact, the background function 'hides' a lot of physics (see Sec. 5.3.5) which, in essence, calls for a more complex formulation of $I^{\text {theo }}(\lambda)$. One is in a much better situation if both analytes combined yield at least one pair of peaks which is close in energy (see also Sec. 5.3.5). To this end, a linear approximation to accommodate unavoidable event-to-event variations in the background is employed:

$$
B G(\lambda)=m \lambda+n
$$

Thus, formally, one deals with a function of four parameters: $I^{\text {theo }}(\lambda)=$ $f(\lambda ; \alpha, \beta, m, n)$. These parameters are obtained by least-squares function minimisation with respect to each experimental spectrum $I_{i}(\lambda)$ :

$$
\min \left[\sum_{\lambda} \sqrt{\left(I_{i}(\lambda)-I^{\text {theo }}(\lambda)\right)^{2}}\right] \Longrightarrow\left\{\alpha_{i}, \beta_{i}, m_{i}, n_{i}\right\} \text {. }
$$

This yields a set of optimal parameters $\alpha_{i}, \beta_{i}, m_{i}, n_{i}$ for each spectrum in the time-series. Based on these fits, the spectral participation of each dye can be redefined as follows:

$$
P_{\mathrm{Rh} 6 \mathrm{G}}=\frac{\alpha}{\alpha+\beta} \quad, \quad P_{\mathrm{BTZ} 2}=\frac{\beta}{\alpha+\beta} .
$$

Note that, since $\alpha$ and $\beta$ are basically scaling factors of the measured reference spectra, this automatically takes care of the difference in Raman cross-section. Specifically, the average spectrum of sol $\mathrm{C}$ leads to factors $\alpha=\beta=1 / 2$, despite the spectrum mostly resembling Rh6G (at least by eye). This procedure thus conveniently normalises the spectral participations with respect to cross sections. 
Figure 5.9 - (A) Scatter plot of the total SERS intensity versus $P_{B}$ obtained from the fits. Note that spectra dominated by one type of dye $\left(P_{B}<0.2\right.$ or $\left.P_{B}>0.8\right)$ are only observed for high intensity events. (B) Histograms of the distribution of $P_{B}$ for solution $\mathrm{C}$, for the simple Poissonian model with $\mu=4$, and for an identical solution with doubled analyte concentration, i.e. $200 \mathrm{nM}$ of each dye. A log scale is used to emphasize extreme events.

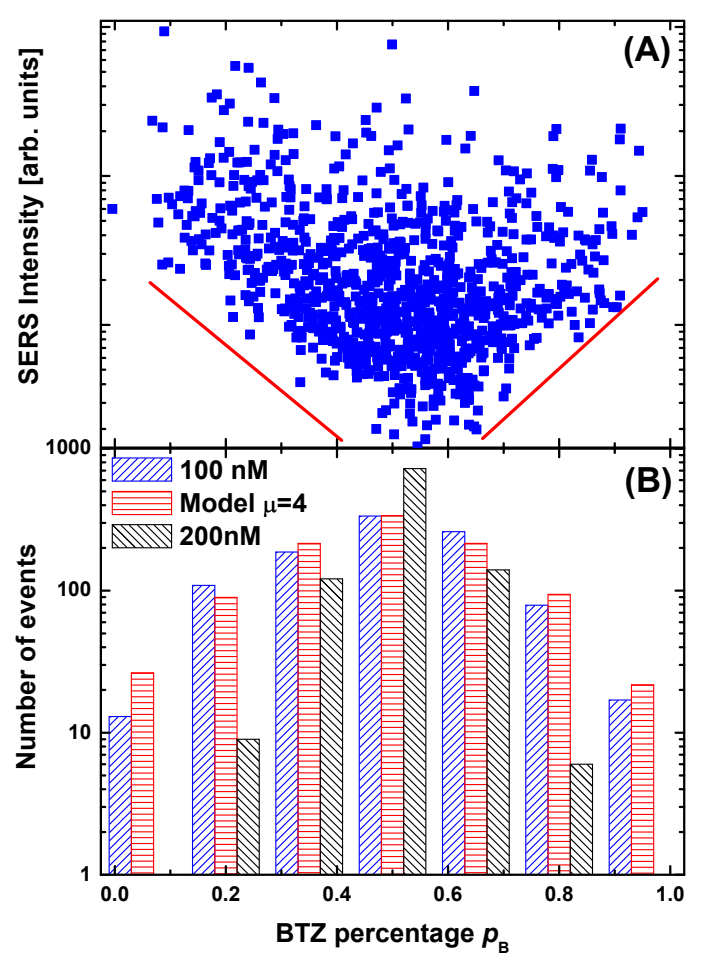

\section{Statistical analysis}

Figure 5.9 shows the results of this analysis; note the logarithmic scale. The top graph plots the intensity of each spectrum (defined as $\alpha+\beta$ ) versus its spectral participation of BTZ2. Evidently, the measurement yields a great variety of participations $P_{\mathrm{BTZ2}}$ - of particular importance are the many cases of $P_{\mathrm{BTZ} 2} \approx 0$ and $P_{\mathrm{BTZ} 2} \approx 1$, since they constitute the experimental SM-SERS proof. Another important pattern visible in this plot is that there are no pure events with low intensities - SM spectra occur only above some intensity threshold. Moreover, there is a clear correlation of spectral participation to minimum intensity (depicted by red lines in the plot) - the less mixed a spectrum, the higher its threshold intensity. This is consistent with the dependency of the enhancement of a hotspot with respect to its 'size', and can be understood in terms of the discrete hotspot model: calculations show [21] that localisation corresponds to enhancement, i.e. the more localised a hotspots, the stronger its enhancement. For 'large' hotspots 
the enhancement is spread out, and hence it is comparatively low for a molecule exposed to it. Furthermore, large hotspots will in general include a large number of molecules, thus making pure spectra improbable, but lend likelyhood to mixed ones. This relation is clearly evident in Fig. 5.9. To summarise this logic: SM signals necessarily originate from a small area, i.e. confined hotspots, which give rise to high enhancements, and thus yield high signal intensities.

The lower plot of Fig. 5.9 collapses the scatter plot into a histogram (blue hatched bars). Note the logarithmic scale. It is clear that the vast majority of events is of a mixed type, but a significant number of pure spectra $(\sim 30)$ is present. Also shown is the fit of an 'open discrete hotspot' model (red hatched bars) to the histogram. The model is an extension of the 'closed discrete hotspot'; it maintains the discrete hotspot, however the number of molecules $N$ contained is no longer fixed, but now Poisson distributed, with separate distributions for each analyte. For a detailed discussion of this model see Appendix B. The fit yields equal means for the number of molecules per hotspot for both analytes, $\lambda_{\mathrm{Rh} 6 \mathrm{G}}=\lambda_{\text {BTZ2 }}=4$, which is (trivially) consistent with the equal dye concentrations and the apparent non-interaction of analytes.

Nonetheless, due to the strong simplifications inherent to this model, the results of this fit have to be taken with care. They do, however, add to the accumulated body of evidence towards SM-SERS. Limitations in mind, one may conclude that on average the signal originates from $\sim 8$ molecules, which translates (assuming two colloids form one hotspot) into an enhancement region which covers on average just $\frac{4}{1200} \approx 0.3 \%$, or $\sim 40 \mathrm{~nm}^{2}$, of the surface of each colloid. This is roughly consistent with previous estimates: in Sec. 3.1.5, it is estimated that $\sim 80 \%$ of the signal stems from $\sim 0.07 \%$ of the surface area. 

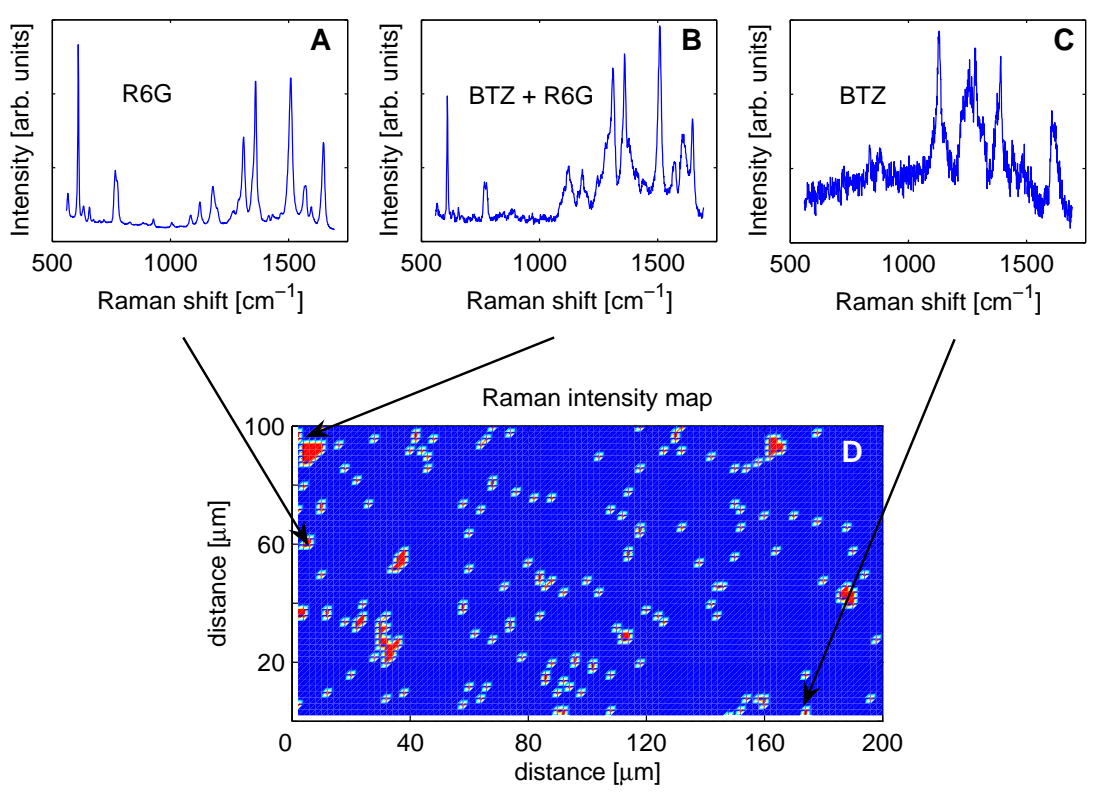

Figure 5.10 - BiASERS of Rh6G and BTZ2 on a dry substrate. (top) Exemplary individual spectra composed of purely Rh6G (A), both Rh6G and BTZ2 (B), and purely BTZ2 (C). Note that there is on average $\sim 500$ molecules of each type per cluster. (bottom) SERS intensity map showing isolated clusters. Red (blue) represents high (low) SERS intensity regions. See text for details.

\section{BiASERS on a dry sample}

Additionally to the BiASERS measurements in liquid, dry samples were prepared, starting from similar solutions (following the procedures outlined in Sec. 4.3). The dried solution contains $20 \mathrm{nM}$ of both Rh6G and BTZ2, i.e. five times less than in the previously studied liquid sample. The idea here was to shift the probability distribution towards pure spectra, while decreasing mixed ones. A lateral scan of the substrate over a $200 \times 100 \mu \mathrm{m}^{2}$ rectangular grid was conducted, with stepsize $2 \mu \mathrm{m}$ in both directions, yielding an overall 5000 spectra. A $\times 100$ air objective was used. The sample was excited at the same wavelength as above $(633 \mathrm{~nm})$, but at a power decreased to $0.2 \mathrm{~mW}$ (ten times less than in the liquid sample case). Each spectrum was taken with an integration time of $1 \mathrm{~s}$ (five times more than in the liquid sample case). 
Figure 5.10 summarises the measurement on the dry sample. The top of the figure shows spectra from the analytes as found in the map, indicating that the BiASERS concept is preserved here - both, BTZ2 and Rh6G, separately and in mixed spectra are observed. The bottom plot shows a colour-coded map of the intensity of each spectrum ${ }^{7}$. It appears that a large number of SERS active sites are present.

Upon closer inspection, however, it becomes clear that the vast majority of intense spectra stem from amorphous-carbon species. Strikingly, the number of discernable spectra here is extremely low - out of 5000 spectra only $\sim 30$ show a spectrum which is attributable to the analytes. At least $50 \%$ of the sites only show a background signal. This is obviously inconsistent with the previous measurement on liquid samples: analyte spectra were abundant and amorphous carbon was absent. How can this be understood?

As discussed in Sec. 4.3, with this method of dry sample preparation the surface density of small colloidal clusters on the substrate can be expected (as an order-of-magnitude estimate) to be $\sim 1$ cluster $/ \mu \mathrm{m}^{2}$. The laser focus on the surface is diffraction limited, thus the surface collected from in each measurement is roughly: $A_{\text {focus }}=4 \pi(\lambda / 2)^{2} \approx 1.3 \mu \mathrm{m}^{2}$. Thus, the estimated cluster density yields an average of $\sim 1$ cluster per laser spot.

Therefore, it is expected to see analytes most of the time: The concentration of $20 \mathrm{nM}$ per analyte translates into $\sim 250$ molecules per colloid. In the liquid case, at a five times higher analyte concentration, the simple Poisson-model (see above) yields an estimate of 4 molecules per hotspot per analyte. Therefore, with a five-fold reduction in analyte concentration, many hotspots will remain unpopulated. Clearly, this should still yield at least one molecule for the majority of hotspots, and can thus not (alone) explain why most measurements yield unattributable spectra.

The effect which curbs the SERS signal on the dry sample is photobleaching.

\footnotetext{
${ }^{7}$ The intensity used in the map was defined as the sum of the data points of the background-corrected spectrum.
} 
The most salient differences between the dry and the liquid substrate occur for the parameters which steer the efficacy of the bleaching effect - most importantly: the presence of molecular oxygen. Photobleaching profits from oxygen ${ }^{8}$; if the dry sample is observed in a $\mathrm{N}_{2}$ atmosphere, the decay of analyte signal towards amorphous carbon is slowed.

Bleaching through oxygen is, however, not the only pathway to the photochemical destruction of the analytes. Another bleaching pathway is simply the extended exposure to high field intensities. In the liquid, colloidal clusters undergo Brownian motion, and, due to their absorbance colloids experience radiation pressure [137, 138, 139], are pushed along the direction of wave-propagation $\vec{k}$, i.e. downwards in conventional laser microscopy geometries. Thus, due to diffusion and laser forces, colloidal clusters may spend only a fraction of the integration time in the laser focus, i.e. in the scattering volume. Consequently, it is hard to judge in which time-frame a spectrum occurs - the integration time merely provides an upper boundary. In the dry sample case however, the clusters remain static to the beam and are exposed to it throughout the entire integration time. The photon flux through molecules in hotspots is then, for the particular conditions chosen here ${ }^{9}$, much larger then in the liquid case.

In conclusion on these remarks, photobleaching explains the large number of intense amorphous carbon spectra. It is therefore imperative to adjust excitation power and integration time for the loss in colloid mobility - one cannot measure liquid and dry samples keeping those parameters fixed, unless the integration time is much shorter than the diffusion time. The principles of BiASERS can be studied on dry substrates, but particular care must be taken to account for photobleaching ${ }^{10}$.

\footnotetext{
${ }^{8}$ Molecular oxygen is a spin-triplet in its ground state; if a dye is resonantly excited (usually $S_{0} \rightarrow S_{1}$ ), it has a probability (through spin-orbit coupling) to shift into a triplet state as well, making energy transfer to the $\mathrm{O}_{2}$ possible. From there a cascade of oxygen radicals can result, which are very reactive.

${ }^{9}$ Laser intensity $(0.2 \mathrm{~mW})$ and integration time $(1 \mathrm{~s})$ combined lead to a photon flux reduction by a factor of 2 with respect to the liquid sample study.

${ }^{10}$ Note that photobleaching can also yield valueable insight on the enhancement distri-
} 


\subsubsection{Caveats}

The BiASERS technique clearly provides a much more rigorous SM-SERS proof than previous attempts. Nonetheless, it is worth mentioning a few caveats and experimental difficulties which -unless addressed- limit the applicability of the method.

\section{Molecular aggregates}

BiASERS is footed on a simple probabilistic proof: if one observes pure spectra, they must come from single, or at most a few, molecules. Pure spectra are highly unlikely to stem from many molecules. This probabilistic argument does not, however, consider the possibility of molecular aggregates. What if, in the colloidal sol, analyte molecules tend to aggregate? Then, in principle, pure spectra could have a large probability of occurrence even when large numbers of (aggregated) molecules are involved. This would void the probabilistic argument of BiASERS.

One cannot simply rule out the formation of molecular aggregates based on the fact that SM-SERS relies on low concentrations. Although in solution aggregation is often diffusion-limited [141], the formation of molecular aggregates depends on the molecule's environment - the metal surface may favour molecular aggregation. Principally, one could test for molecular aggregates using absorption [142] or fluorescence spectroscopy [143], however, under SM-SERS conditions these techniques are hard to apply since (i) the analyte's absorption is hidden within the colloidal absorption spectrum (due to the extremely low analyte concentration) and (ii) fluorescence under SERS conditions is already heavily modified ${ }^{11}$. It is also hard to judge from the Raman spectrum, since the forces binding molecules into aggregates are usually small and thus in general do not impact polarisabil-

\footnotetext{
bution, see Ref. [140].

${ }^{11}$ The interaction with the metal surface and the plasmonic resonance subject the molecule's fluorescence to wavelength-dependent quenching and enhancement [68].
} 
ity much - also, the Raman spectrum is in many cases already modified under SERS conditions (w.r.t. normal Raman) and a powder spectrum for comparison is not always available.

However, molecular aggregates in BiASERS are readily refuted in the statistical analysis of the BiASERS results itself. For example, in a one-to-one mixture of two analytes, if one analyte were prone to aggregation, then events involving this dye would be more sparse than the other; hence, the event histogram would be skewed towards one dye. This argument holds unless both dyes have the exact same aggregation dynamics. Since this has not been observed for the range of BiASERS partner analytes studied in our group and in the recent literature, it appears conclusive that molecular aggregates are not a general problem in BiASERS, but rather an oddity which might occur in some special cases.

Consequently, this discussion results in an application of Occam's razor: the simplest explanation (i.e. single molecules), holds unless a contradiction is encountered. After all, for most molecules aggregates are not a problem.

\section{Plasmonic background}

As exemplified in Eq. 5.6, the determination of spectral participances requires knowledge about the SERS background, $B G(\lambda)$. This background is thought to be caused by modified fluorescence [68], whereas the spectral variation attests the fluctuation of the underlying plasmonic resonance [144]. In view of the physics involved, explicitly accounting for the background will demand approximations. In principle, one could simply assume a polynomial of sufficiently high order to represent the background, however, that would only improve the fit at the cost of its significance.

A more suited approach avoids the problem altogether - instead of comparing entire spectra, a small spectral window in which both analytes have 
at least one non-overlapping peak (to allow uneqivocal identification of analytes) becomes the center of investigation. If the window is sufficiently small, the background may be assumed constant across it. As an example, the dyes Rh6G and Nile Blue (NB) are a well-suited BiASERS pair since they have strong Raman peaks at $612 \mathrm{~cm}^{-1}(\mathrm{Rh} 6 \mathrm{G})$ and $590 \mathrm{~cm}^{-1}(\mathrm{NB})$, thus reducing the required window for analysis to $\sim 30 \mathrm{~cm}^{-1}$ spectral width. Since plasmonic resonances typically occur on a much larger scale [144], one can safely assume $B G$ to be a constant in this case.

\subsubsection{BiASERS applications}

The BiASERS technique to identify SM-SERS has found adaptation in the SERS community, see for example Refs. [127, 27, 145]. This section shall not dwell on these, but rather highlight a number of relevant publications to which I contributed during the course of my work for this thesis.

\section{Static or dynamic aggregates?}

A pending question in chapter 4 was whether colloidal aggregates are static or dynamic, i.e. once formed, do colloids stick permanently or is it possible for them to split and re-form new colloidal aggregates? This question can be answered using the BiASERS technique: two samples are prepared:

sample A: prepared by mixing two pre-aggregated, i.e. SERS-active, samples of BTZ2 and Rh6G

sample B: a classic BiASERS sample in which both dyes (BTZ2 and Rh6G) are added to the colloidal sol first, then aggregation is induced by adding $\mathrm{KCl}$

All other sample conditions (concentrations etc.) are kept constant. Figure 5.11 plots the Raman intensity (on a logarithmic scale) of samples A (top) and B (bottom) against the participance of one of the analytes (here BTZ2). If 


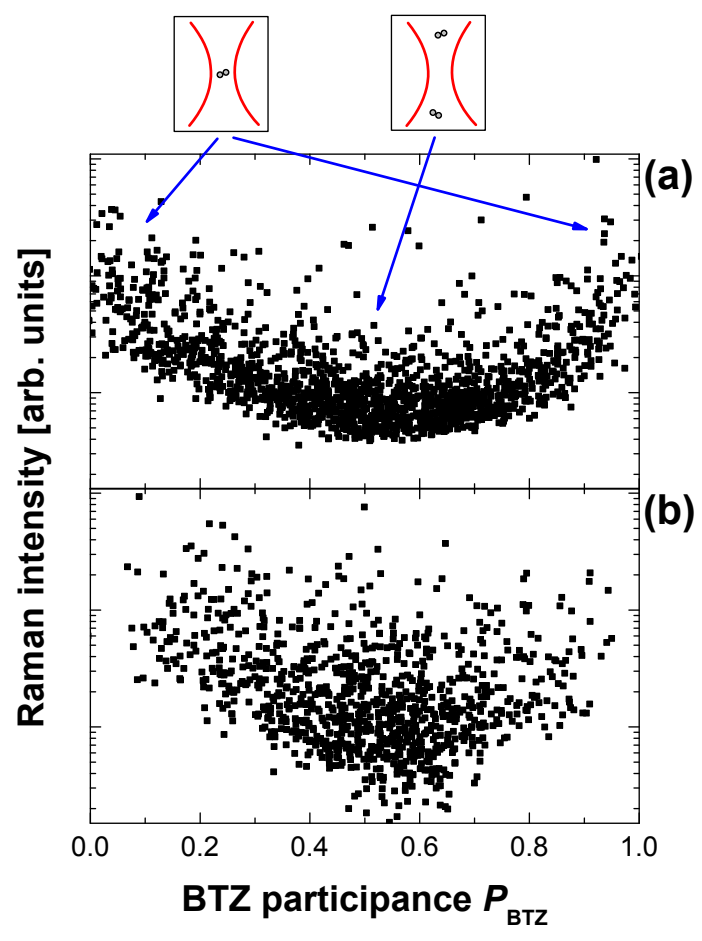

Figure 5.11 - Correlation plots of intensity vs dye participation $\left(P_{\mathrm{BTZ}}\right)$ for sample A (top plot) and, for contrast, sample B (bottom plot). The lack of high intensity mixed events for sample $\mathbf{A}$ proves that the colloidal clusters are static. Note the log-scale in the intensity axes. See text for further details

colloidal clusters were to form (and split up) dynamically, then both samples should be indistinguishable (picture, for example, same-dye colloidal aggregates in sample $\mathbf{A}$ to split up and re-form into mixed-dye colloidal aggregates, as present in sample $\mathbf{B})$. This is, evidently, not the case. The banana-like shape of the plot in the case of sample $\mathbf{A}$ is easily understood: pure spectra of high intensity happen frequently, consistent with same-dye hotspots having statically formed before the two SERS samples were mixed. Events with signatures of both dyes appear only at low intensities, which is expected if more than one hotspot is observed during the integration time. The lack of high intensity mixed-dye events in sample A proves that colloidal clusters are static, as expected from DLVO theory. Moreover, a qualitatively similar plot (not shown) is obtained if the sample is left to age 

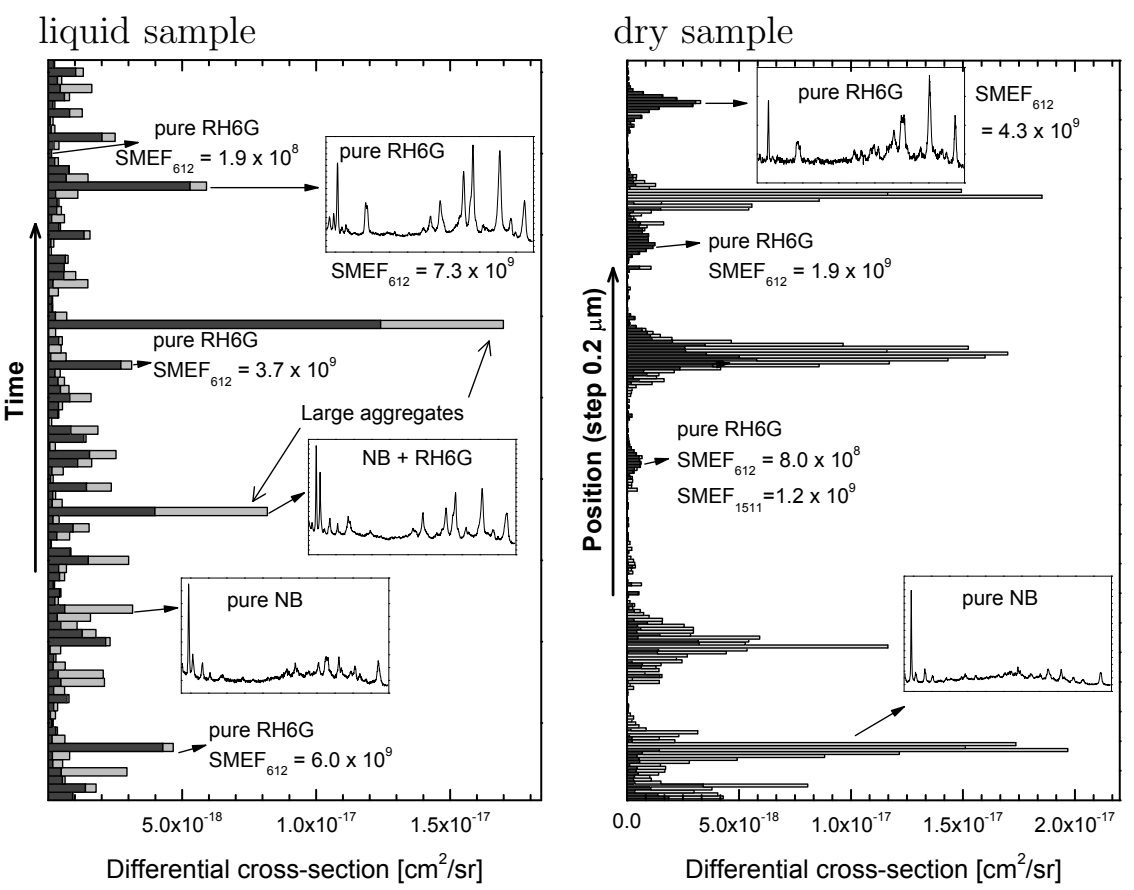

Figure 5.12 - Cross-section and relative signal participation of (left) a timeseries of BiASERS spectra from a liquid sample and (right) a spatial scan of the dried sample. Nile Blue and Rh6G (concentration of $1 \mathrm{nM}$ each) were used with standard Ag colloids. See text and Ref. [21] for details.

for one day, hence indicating the long-term stability of the clusters.

\section{Single-molecule enhancement factor}

As demonstrated by Le Ru et al. [21], with the full characterisation of the analytes (i.e. measuring the bare Raman cross-section) and the scattering volume (see supporting information of Ref. [21]) it becomes possible to deduce the SMEF (see Sec. 3.1.1, Eq. 3.6, and also Refs. [21] and [17]). Figure 5.12 shows exemplary results from series of spectra taken in liquid (left plot) and on dry substrates (right plot). Strikingly, the smallest and largest detectable SM signals yielded SMEFs ranging from $10^{8}$ to $10^{10}$, thus falsifying the conventional assumption of $\sim 10^{14}$. 


\section{Isotopically edited analytes}

One of the more profound advances in the BiASERS technique comes through using isotopically edited dyes as BiASERS partner analytes. First used in SERS for quantitative analysis [136, 146], isotopically edited Rh6G was mentioned [147] and later published [145] by Dieringer et al. as a means to identify SM-SERS signals. The advantage lies in the obvious chemical similarity of both analytes: by replacing a fixed set of hydrogen atoms with deuterium, the geometry, surface adsorption, etc... can be expected to be largely the same for both molecules. However, the frequency of the vibrations in which the hydrogen/deuterium atoms take part is altered, and hence, the two versions of the molecule become distinguishable. Following this approach, Blackie et al. [148] synthesised and characterised Rh6M and d4-Rh6M (a close cousin of Rh6G, see Fig. 5.13) and employed these analytes to investigate the experimental parameters (scattering volume, integration time, dry/liquid substrate, etc...) which are important for the observation of SM-SERS events. Fig. 5.13 summarises the spectral differences exhibited by d4-Rh6M and Rh6M. From the average spectra, the most notable spectral changes are confined to two regions (see plots (b) and (c)). In region $\mathrm{I}$, the prominent mode at $610 \mathrm{~cm}^{-1}$ shifts by $\sim 10 \mathrm{~cm}^{-1}$ upon deuteration. In region II, the Rh6M doublet at $1310 \mathrm{~cm}^{-1}$ and $1365 \mathrm{~cm}^{-1}$ splits into a triplet for d4-Rh6M. Fig. 5.14 shows SM spectra (in regions I and II) attributable to Rh6M and d4-Rh6M, as well as an exemplary mixed spectrum. For comparison, the average spectra are shown as well (bottom plot).

Isotopically edited dyes do not provide a qualitatively better proof of SMSERS; their advantage lies in their chemical similarity. This enables one vary conditions and parameters (different substrates, $\mathrm{KCl}$ concentrations, etc..) without causing uncertainties in the contrast provided by the BiASERS partners (which potentially would behave differently under varied conditions). Hence, isotopically edited dyes are a convenient tool to study SM-SERS (see, for example, Ref. [140]). 


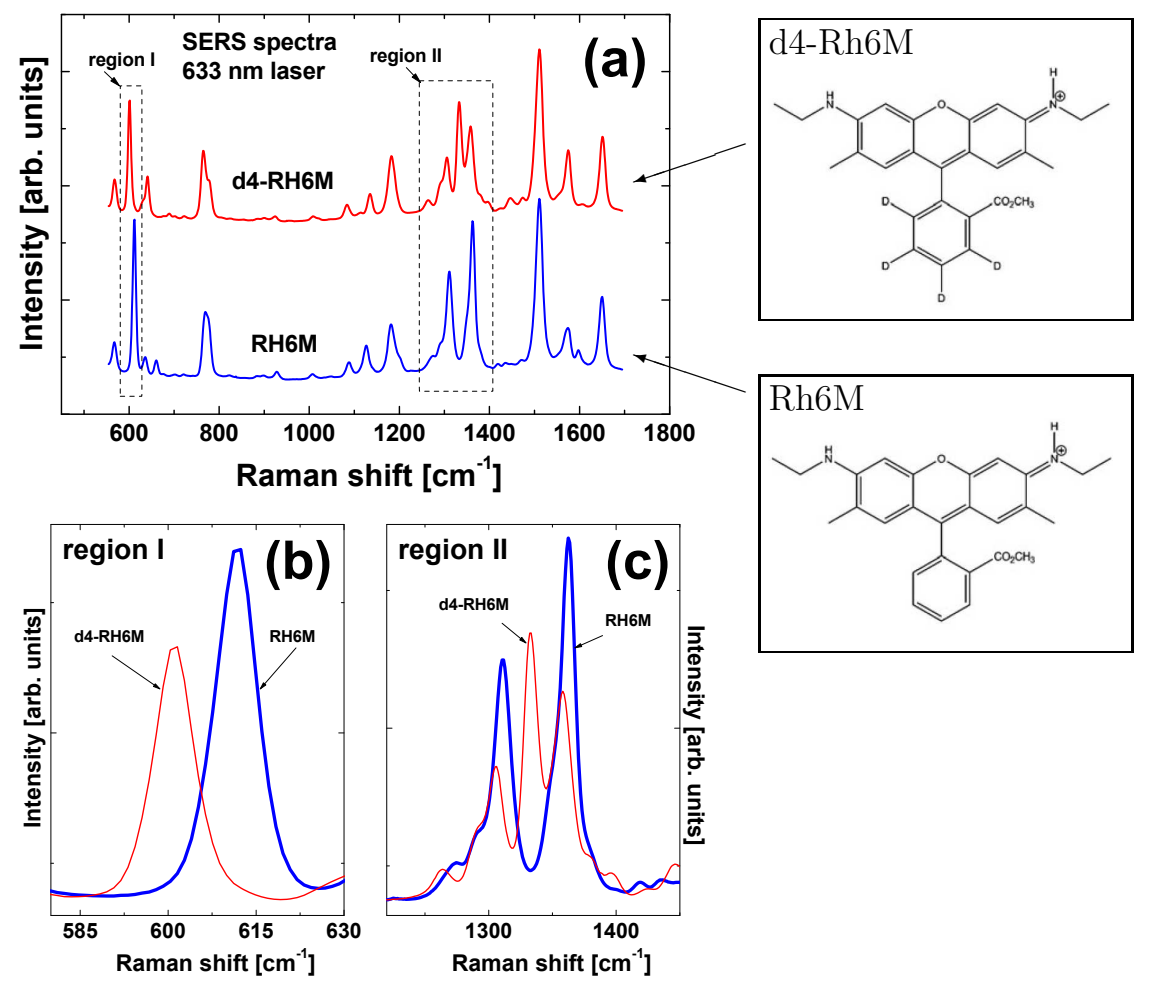

Figure 5.13 - (a) SERS spectra of d4-Rh6M and Rh6M at $100 \mathrm{mM}$ concentration in Lee \& Meisel Ag colloids with $10 \mathrm{mM} \mathrm{KCl}$. The measurements correspond to the average of $10^{3}$ spectra with $1 \mathrm{~s}$ integration time. (b) Region I and (c) region II show distinct spectral differences produced by isotopic substitution. The Lewisstructures of d4-Rh6M and Rh6M are given on the right. See text and Ref. [148] for details.

\section{Detection of natural isotopes}

Along the lines of purposely isotopically edited analytes, it is also possible to discern, in some cases, the isotopic shift caused by the natural abundance of atoms - this is the topic of Ref. [149]. A peculiar novum here lies in the fact that only one analyte is added, which becomes its own BiASERS partner, through the occasional presence of a different isotope within the molecule. Figure 5.15 (a) shows the structure of Rhodamine 800, the molecule used in this experiment. Part (b) highlights the cyano bond, which is of particular interest here due to its isolation in vibrational coupling and frequency 


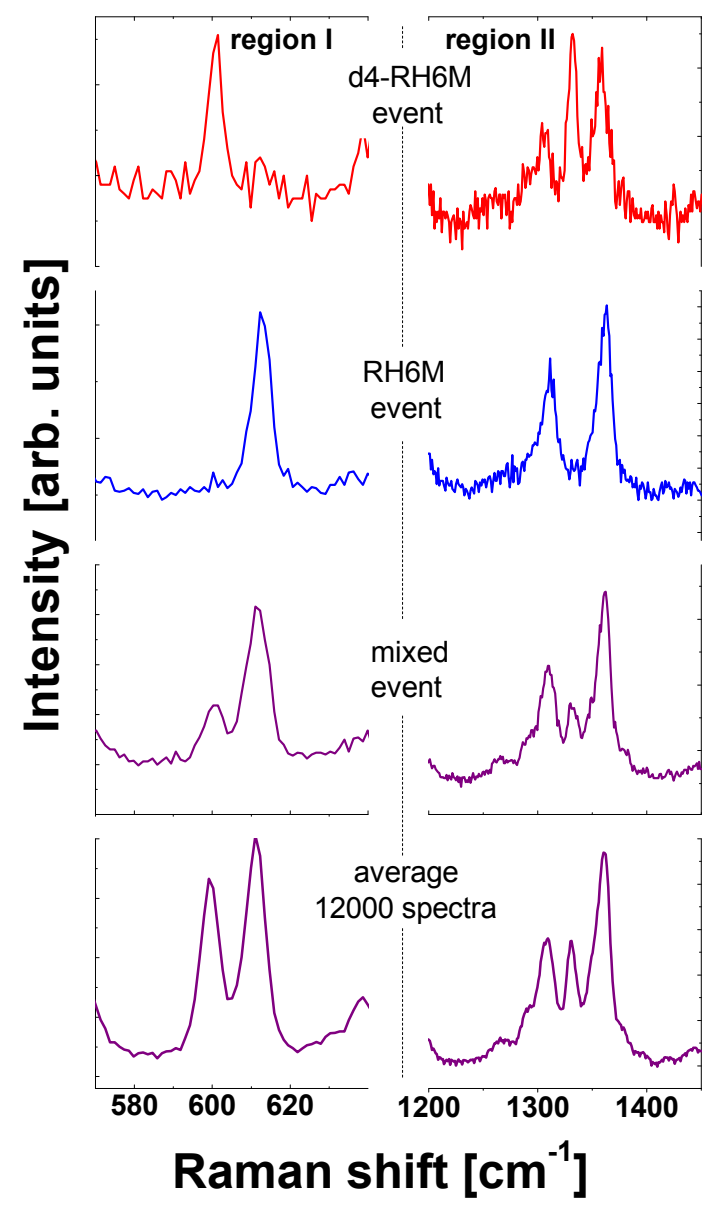

Figure 5.14 - Single molecule SERS events for d4-Rh6M and Rh6M together with a mixed d4Rh6M/Rh6M event. The average spectrum for $12 \times 10^{3}$ spectra taken at different integration times is also shown at the bottom. See text and Ref. [148] for details.

with respect to the remainder of the molecule: the triple bond oscillates in the normally Raman-silent region, at $2230 \mathrm{~cm}^{-1}$, depicted in part (c) of the figure. In view of the cyano bond, one notes that carbon has a small natural isotopic spread [150] between ${ }^{12} \mathrm{C}(98.9 \%)$ and ${ }^{13} \mathrm{C}(1.1 \%)$, and nitrogen is even less spread at $99.63 \%$ for ${ }^{14} \mathrm{~N}$ and $0.37 \%$ for ${ }^{15} \mathrm{~N}$. Nonetheless, in a large time-series (here, 18000 spectra) these minute abundances are indeed detectable (note that PCA ${ }^{12}$ was used to remedy the cumbersome analysis of this large number of spectra). Figure 5.16 shows a selection of representative spectra taken from the time-series, showing shifts in the vibrational frequency of the cyano bond consistent with the shifts expected

\footnotetext{
${ }^{12}$ Principal Component Analysis, as described in the BiASERS context in Ref. [113], as well as in the supplementary information of Ref. [149].
} 


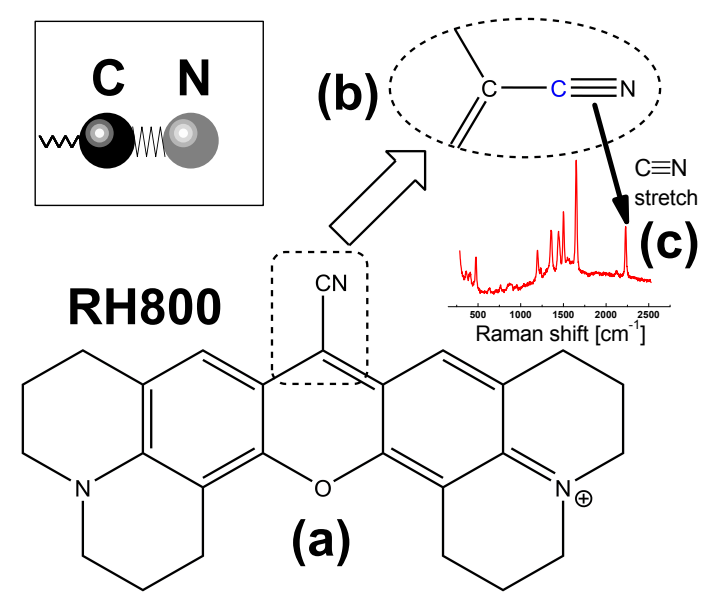

Figure 5.15 - (Top left inset) schematic of the cyano bond in Rhodamine 800 (Rh800). Note that the triple bond corresponds to a force constant ('spring') much larger than the single bond coupling the cyano group to the rest of the molecule. (a) Lewis-structure of Rh800, (b) the cyano bond in its local group and (c) SERS spectrum of Rh800. The highlighted stretch of the triple bond is (in the average case) at $2230 \mathrm{~cm}^{-1}$.

from ${ }^{13} \mathrm{C}$ and ${ }^{14} \mathrm{~N}$ (the magnitude of the shifts were predicted using Density Functional Theory (DFT), see Sec. 6.4, as well as the supporting information to Ref. [149].)

\subsection{Conclusion}

In conclusion, the BiASERS method is a powerful technique for the identification of SM-SERS events. By exploiting the strong localisation of the enhancement in SERS, it allows for comparatively large surface concentrations and, hence, yields abundant events. In turn, statistical assertions become reliable. Apart from its initial conception, i.e. to provide an unambiguous proof of the SM nature of signals under certain SERS conditions (SERS of resonant dyes in Lee \& Meisel Ag colloids), the technique represents a general tool useful for the study of SM-SERS in different systems (i.e. substrates, analytes, excitation wavelength, etc...); in particular, it is 


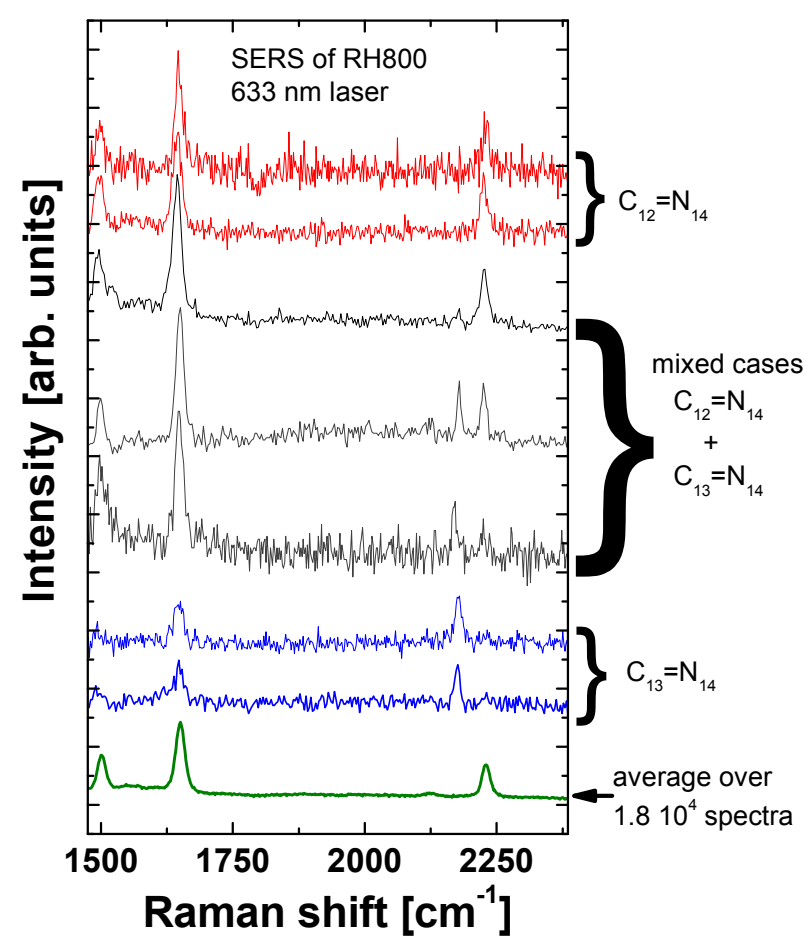

Figure 5.16 - Exemplary selection of spectra from the time-series of 18000; all spectra are displaced for clarity. Top two spectra (red) show the most abundant case: ${ }^{12} \mathrm{C}-$ ${ }^{14} \mathrm{~N}$. The three central spectra (black) show mixed cases in which both, the most abundant and the case ${ }^{13} \mathrm{C}-{ }^{14} \mathrm{~N}$ are visible. Pure versions of the latter case are shown below (blue). The average spectrum of the entire time series is plotted at the bottom (green).

useful to evaluate whether a SERS system is capable of providing SM signals under the given conditions, or not. To this end, the importance of chemically similar dyes (see above, discussion on isotopically edited dyes) was highlighted in this context. 


\section{Chapter 6}

\section{Quantum chemistry}

After a number of decimal places, nobody gives a damn.

unknown

As a complement to the preceding, largely experimental chapters, this part of the thesis will introduce and exemplify the specific applications density functional theory (DFT) has within the realm of SERS. Although not directly connected to the experiments discussed in-depth in this thesis, this theoretical approach was nonetheless employed heavily throughout the course of the work undertaken for this thesis, and has contributed to publications $[149,72,21]$. It is also worth pointing out the educative power of quantum chemistry - through its use one inevitably gains insights on the more intricate molecular workings, e.g. the molecular dipoles and symmetry of Raman tensors. For more than 30 molecules the geometries and Raman spectra were simulated during this work; often simply to gain some confidence in an experimental result with a (semi) ab-initio calculation, but also to conduct tests deciding whether a full experimental investigation is worth its time. In this sense, a large part of the work done in DFT is 'silent' and often goes unnoticed. In response, this chapter shall point out some of the capabilities of DFT in SERS, with a focus on the 
physical entity governing the effect studied here - the Raman tensor.

\subsection{Introduction}

$\mathrm{Ab}$ initio methods and DFT have become of age in the prediction of various physical and chemical molecular properties (e.g. geometry, dipole moment, excited states, reaction constants). Continuous refinement of basis functions [151, 152, 153,154] and energy functionals [155], together with the ever-rising contemporary computing power [156], enables one to model the electronic molecular orbitals with sufficient accuracy to calculate second order derivatives of the potential energy surface (with respect to atom positions) and first order polarisability derivatives (with respect to normal modes of vibration) - in short, the predicament to the simulation of Raman spectra. Such predictions lend themselves as powerful tools for:

a) the identification of unknown molecular species,

b) the prediction of Raman spectra (e.g. predicting the effect of isotopic substitution), and, hence,

c) aiding rational synthesis of SERS probes.

Yet, vibrational spectroscopic properties calculated by modern quantum chemistry software $[157,158]$ constitute a largely unfathomed treasure trove. For example, traditional normal mode analysis had to rely on empirical (bond-)polarisabilities and point-group analyses of often inaccurate geometries. On the other hand, today, one readily computes the normal modes right down to the atomic displacement vectors, which, at least in specific cases, can yield valuable insights (see Sec. 6.4).

In analogy, in the past Raman tensors were studied largely for their symmetry (i.e. depolarisation ratio). Today, one can go beyond the ensemble notion of Raman tensor invariants and gain insights from analysing Raman 
tensors directly in any particular spatial frame. Aided by an appropriate tensor representation (see below), it becomes possible to analyse and compare individual tensors and study their relation to experiment. Moreover, some limited insights into the effects of resonance conditions on the tensors are gained.

The chapter is structured as follows: Sec. 6.2 will review the required theoretical background, followed by an overview of computational model in Sec. 6.3. Sec. 6.4 will tersely discuss some exemplary applications of DFT undertaken during this work. Sec. 6.5 provides some specific guidance on the stony path of Raman tensor calculation. I will propose a visual tensor representation designed for the SERS/TERS case in Sec. 6.6, and, building on this visualisation, Sec. 6.8 will discuss insights about surface selection rules and molecular orientations. In Sec. 6.9 this is extrapolated to resonance induced tensor modifications, which predicts a selection rule breakdown in SM-SERS under resonance conditions.

\subsection{Physical background}

The classical treatment of the Raman effect can be found in any textbook concerned with the matter $[3,16,159,17]$ and was touched in Sec. 2.2. Nonetheless it is beneficial to re-state some basic connections and formulae here for clarity, with an emphasis on the details of DFT. The computations described in this chapter are based directly on these foundations.

Any vibration can be decomposed into the molecule's vibrational normal modes, $\vec{q}_{i}$ (see Refs. $[159,18]$ ). This basic fact translates into the following computational scheme:

1. The Hessian matrix is formed, which is the $3 \mathrm{~N} \times 3 \mathrm{~N}$-matrix of second derivatives of the potential energy surface with respect to the coordinates of the $N$ atoms constituing the molecule, $\frac{\partial^{2} V}{\partial \vec{r}^{2} \partial \vec{r}}$. 
2. The Hessian matrix is diagonalized by the coordinate system transformation $\vec{r}_{i} \rightarrow \vec{q}_{i}$ which yields the eigenvectors $\vec{q}_{i}$ (normal modes) and eigenvalues $k_{i}$ (force constants).

3. Vibrational frequencies are then determined via $\omega_{i}=\sqrt{\frac{k_{i}}{\mu_{i}}}$, where $\mu_{i}$ is the (weighted) reduced mass of the vibration.

4. The lowest 6 (or 5 , see Fig. 2.2) positive frequencies correspond to motion and rotation of the rigid molecule,

5. Negative values of $\omega_{i}^{2}$ (i.e. imaginary frequencies) indicate transition states.

The resulting normal modes can be visualized as a set of displacement vectors; one for each atom with respect to its equilibrium position.

For a mode $m$, all atoms displace in phase, such that it can be described by a single scalar, oscillatory function $Q_{m}(t)$. Thus the position $\vec{r}$ of the atoms can be rewritten as $\vec{r}=Q_{m} \vec{q}_{m}$. For small departures $Q_{m}$ from the atoms' equilibrium positions, the potential energy surface $V$ is approximately quadratic, i.e. the molecule behaves like a harmonic oscillator. The dipolar response of a molecule to an electric field $\vec{E}$ is formally described by $\vec{p}=\hat{\alpha} \vec{E}$, where $\vec{p}$ is the induced dipole and $\hat{\alpha}$ is the $3 \times 3$ polarisability tensor. The Raman effect is the response of $\hat{\alpha}$ to $Q_{m}$ - a Taylor expansion of the components $\hat{\alpha}_{\rho \sigma}$ with respect to $Q$ yields, in analogy to Eq. 2.5:

$$
\begin{aligned}
\hat{\alpha}_{\rho \sigma}=\left(\hat{\alpha}_{\rho \sigma}\right)_{0} & +\sum_{m}\left(\frac{\partial \hat{\alpha}_{\rho \sigma}}{\partial Q_{m}}\right)_{0} Q_{m} \\
& +\frac{1}{2} \sum_{m, n}\left(\frac{\partial^{2} \hat{\alpha}_{\rho \sigma}}{\partial Q_{m} \partial Q_{n}}\right)_{0} Q_{m} Q_{n} \ldots,
\end{aligned}
$$

which contains the Raman polarisability tensor components

$$
\hat{R}_{\rho \sigma}^{m}=\left(\frac{\partial \hat{\alpha}_{\rho \sigma}}{\partial Q_{m}}\right)_{0} Q_{m}
$$

for the normal mode $m . \hat{R}$ has two notable invariants, the isotropic part:

$$
\bar{\alpha}=\frac{1}{3}\left(\hat{R}_{x x}+\hat{R}_{y y}+\hat{R}_{z z}\right),
$$


and anisotropic part:

$$
\begin{aligned}
\gamma^{2}=\frac{1}{2}\left[\left(\hat{R}_{x x}-\hat{R}_{y y}\right)^{2}+\left(\hat{R}_{y y}-\hat{R}_{z z}\right)^{2}\right. & \\
& \left.+\left(\hat{R}_{z z}-\hat{R}_{x x}\right)^{2}\right]+3\left(\hat{R}_{x y}^{2}+\hat{R}_{x z}^{2}+\hat{R}_{y z}^{2}\right) .
\end{aligned}
$$

These numbers are invariant under any cartesian transformation (which is why $\hat{R}$ is denoted as a cartesian tensor in the first place). Two ensemble properties are defined by the invariants: (a) the Raman activity $R A$ and (b) the depolarisation ratio $\rho$. The former is given by ${ }^{1}$ :

$$
R A=\frac{45 \bar{\alpha}^{2}+4 \gamma^{2}}{45}
$$

and the latter is defined as:

$$
\rho=\frac{3 \gamma^{2}}{45 \bar{\alpha}^{2}+4 \gamma^{2}}
$$

The Raman activity is proportional to the absolute differential Raman crosssection $\frac{d \sigma}{d \Omega}$ (and thus to the Raman intensity). However, this magnitude also depends on the laser and normal mode frequencies $v_{L}$ and $v_{m}$, as well as on the populations of the vibrational levels. A full account of the derivation of the Raman intensity/cross-section has been done elsewhere (see for example Neugebauer et al. [160] and Le Ru and Etchegoin [17]); the absolute differential Raman cross-section reads:

$$
\frac{d \sigma}{d \Omega}=\frac{\pi^{2}}{\varepsilon_{0}^{2}}\left(\bar{v}_{L}-\bar{v}_{m}\right)^{4} \frac{\hbar}{4 \pi c \bar{v}_{m}} \frac{R A}{1-\exp \left(\frac{-h c \bar{v}_{m}}{k_{B} T}\right)},
$$

where $\bar{v}_{L}$ and $\bar{v}_{m}$ are the absolute wavenumbers of the laser and the $m$ th vibrational mode.

These quantities (Eqs. 6.5, 6.6, 6.7) are useful when compared to the results of normal Raman experiments (see, for example, supporting information

\footnotetext{
${ }^{1}$ Valid for linearly polarised excitation and parallel polarised detection. For unpolarised excitation (lamp) but polarised detection the formula changes to $R A=$ $\left(45 \bar{\alpha}^{2}+7 \gamma^{2}\right) / 45$.
} 
of Ref. [21]). However, due to the implicit orientational averaging in this treatment details are neglected which become meaningful as soon as the focus shifts from a disordered ensemble to a few (or single) scatterers. The Raman tensors yield more information than the ensemble observables of a traditional Raman experiment - and, as shall be shown in the next section, it is instructive to have a detailed look at the tensors in the SERS/TERS context.

\subsection{Computational models}

I shall give a brief and general outline of the two steps typically involved in the calculation of Raman tensors via DFT:

First, the optimal (lowest energy) molecular geometry is determined. Finding the optimal atomic coordinates is a standard task for most DFT software packages, but is not in all cases and easy goal (a good starting guess is essential, but not sufficient ${ }^{2}$ ).

In the second step, the optimized atomic coordinates are used as input for the evaluation of vibrational molecular properties, i.e. -in this case- the Raman response of the molecule to an (oscillating) electric field. Following the normal-mode analysis described above, the distortion of the electron density with respect to the unperturbed molecule is calculated for the case of electric fields applied along the $x, y$ and $z$-axis. Combined with the normal mode displacement pattern this yields the associated change in polarisability, which can be reformulated as the Raman tensor.

In all calculations shown here Becke's 3-parameter hybrid functional [161] with Lee-Yang-Parr's non-local electron correlation [155], commonly abbreviated as B3LYP, was employed. This DFT method operated on an extensive Pople split basis set [152] with diffuse functions, 6-311++G(d,p).

\footnotetext{
${ }^{2}$ As an example, from experience, one encounters convergence problems in the geometry search of azulene.
} 
Note that it is in general mandatory to perform the geometry optimization as well as subsequent analyses using the same combination of method and basis set in order to get consistent results.

\subsection{Applications of DFT}

As stated earlier, much of the work which goes into DFT is not reflected in publications, since it is mostly used as a helper tool aiding the experimental work through simple tests and predictions ${ }^{3}$. Nonetheless, a number of findings are well worth pointing out, and, hence this section will portray some notable applications of DFT. Note however, that the more intricate investigation on the resonance effects shall not be mentioned here, since it requires some preliminary considerations and deserves its own section (see Sec. 6.9).

\section{Surface binding of benzenethiol}

Figure. 6.1 compares the normal Raman spectrum of benzenethiol (BT) to its SERS spectrum (experimental data). Evidently, under SERS conditions the spectrum is heavily modified; bands shift and change relative intensities. However, the somewhat broad band which appears in the normal Raman spectrum at $\sim 930 \mathrm{~cm}^{-1}$ is completely absent in the SERS spectrum. In view of the strong spectral modifications one might argue that this is simply a case in which the intensity of the mode is subdued below the detection limit. This turns out to be true, but for a very straight-forward reason: the inset in Fig. 6.1 shows the vibrational displacement pattern of the $930 \mathrm{~cm}^{-1}$ mode, as obtained from DFT. Clearly, this mode is extremely localised in the molecule and basically is solely the wagging of the hydrogen atom in the thiol group. To a chemist, it will come as no surprise that

\footnotetext{
${ }^{3}$ To quote Fairthorne [74] once more: "Often all that is needed is reasonable assurance that water will flow into the sink and not on to the ceiling."
} 


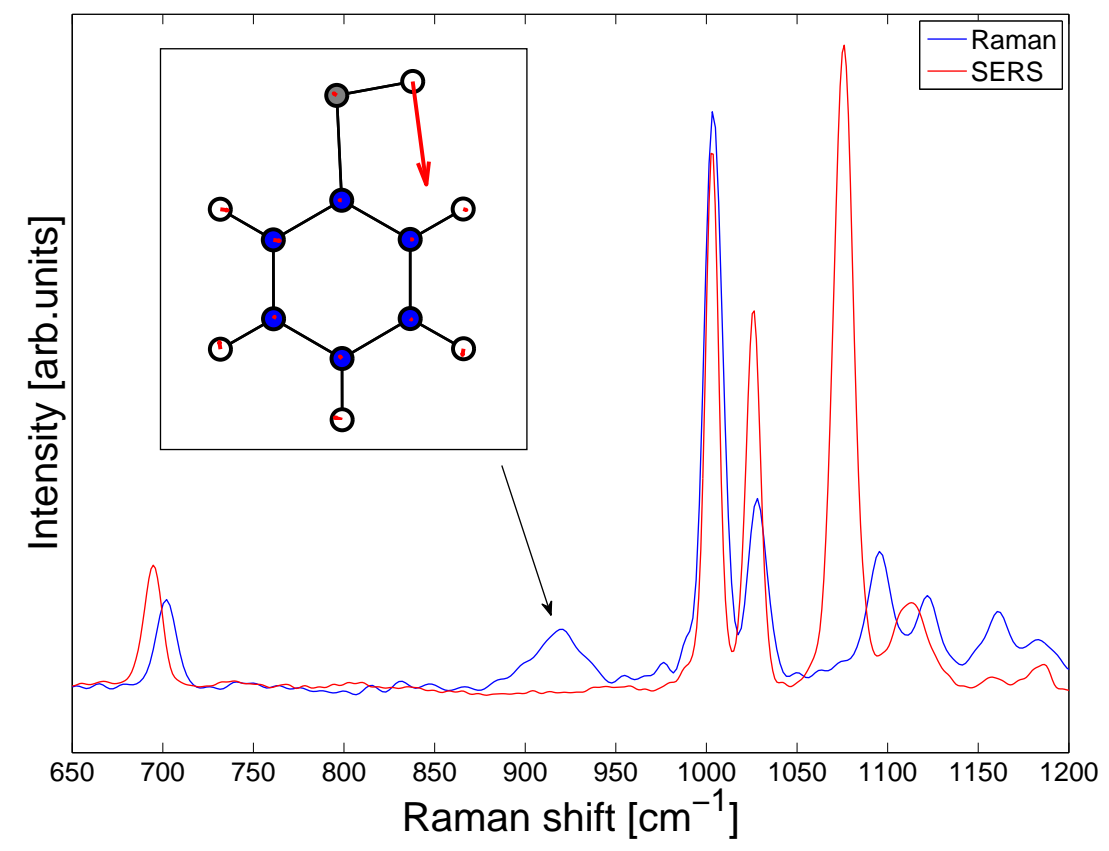

Figure 6.1 - Proving the surface attachment of benzenethiol (BT) using DFT. The main plot shows the Raman spectrum of an aqueous BT solution (solid line) and the SERS spectrum of BT in an aqueous Ag-colloid suspension (dotted line). The inset annotates the mode at $\sim 930 \mathrm{~cm}^{-1}$ with its calculated atomic displacement pattern (B3LYP functional, 6-311++G(d,p) Pople basis set). The involvement of the thiol group in the attachment to the $\mathrm{Ag}$ surface becomes apparent in the complete disappearance of the mode in the SERS spectrum.

BT binds through this group to the surface by forming a covalent bond between sulfur and silver, while sacrificing the hydrogen. Thus understandable, the $930 \mathrm{~cm}^{-1}$ vibration is not present in the surface complex at all. Inverting this logic, since the vibration is only a wagging in the thiol group, and since it is absent under SERS conditions, the thiol group must be strongly altered under SERS conditions, i.e. it forms the bond. This latter argument requires due care: the phenomenon of binding is not always associated with the absence of a mode in the spectrum, however, the consistency with prior knowledge in this case adds an additional layer of confidence on the accuracy of the DFT eigenvectors. 


\begin{tabular}{cccccccccc}
\hline & \multicolumn{3}{c}{ DFT predictions } & \multicolumn{4}{c}{ Experimental } & \multicolumn{2}{c}{ Reported (Ref. [162]) } \\
\hline $\bar{v}_{i}$ & $\rho_{i}$ & $\left(\frac{d \sigma}{d \Omega}\right)$ & $I_{i}^{\text {Rel }}$ & $\bar{v}_{i}$ & $\rho_{i}$ & $I_{i}^{\text {Rel }}$ & $\left(\frac{d \sigma}{d \Omega}\right)$ & $I_{i}^{\text {Rel }}$ \\
{$\left[\mathrm{cm}^{-1}\right]$} & {$[-]$} & {$\left[10^{-32} \frac{\mathrm{cm}^{2}}{\mathrm{sr}}\right]$} & {$[-]$} & $\mathrm{cm}^{-1}$ & {$[-]$} & {$[-]$} & {$\left[10^{-32} \frac{\mathrm{cm}^{2}}{\mathrm{sr}}\right]$} & {$[-]$} \\
\hline 265 & 0.75 & 25.9 & 0.16 & 280 & 0.70 & 0.17 & - & - \\
293 & 0.26 & 130 & 0.82 & 302 & 0.26 & 0.85 & 144 & 0.85 \\
509 & 0.18 & 159 & 1.00 & 516 & 0.16 & 1.00 & 169 & 1.00 \\
800 & 0.63 & 53.4 & 0.34 & 806 & 0.60 & 0.26 & 44.0 & 0.26 \\
1168 & 0.26 & 60.8 & 0.38 & 1143 & 0.32 & 0.34 & - & - \\
1255 & 0.75 & 10.6 & 0.07 & 1237 & 0.74 & 0.04 & - & - \\
\hline
\end{tabular}

Table 6.1 - Comparison of DFT predictions (gas phase), experimental measurements (liquid phase), and reported values (gas phase) for the different Raman active modes of 2B2MP. Shown are the vibrational Absolute Raman cross-sections and relative intensities all relate to $633 \mathrm{~nm}$ laser excitation.

\section{Cross-section of 2B2MP}

As part of the SMEF cross-section determination (see Sec. 5.3.6 and Ref. [21]), the reference compound 2-bromo-2-methylpropane (2B2MP), for which the Raman cross-section is known from literature [162], was used. However, to gain confidence in the literature values, the Raman cross-section was compared to the theoretical result. In Table. 6.1 the DFT results are compared to experimental as well as literature values for the vibrational frequencies $\bar{v}_{i}$, depolarisation ratios $\rho_{i}$, cross-sections $\left(\frac{d \sigma}{d \Omega}\right)$ as well as relative peak intensities $I_{i}^{\text {Rel }}$. Evidently, as the numbers attest, the DFT results strongly support the literature claims as well as the experimental results. This emphasises the level of accuracy DFT calculations reach with small molecules.

\section{Predicting isotopic shifts}

Isotopic substitution in SERS analytes can yield valuable BiASERS pairs, as was emphasised in Sec. 5.3.6. Instead of measuring the spectra of scores of isotopically substituted analytes, DFT can be employed to predict the 

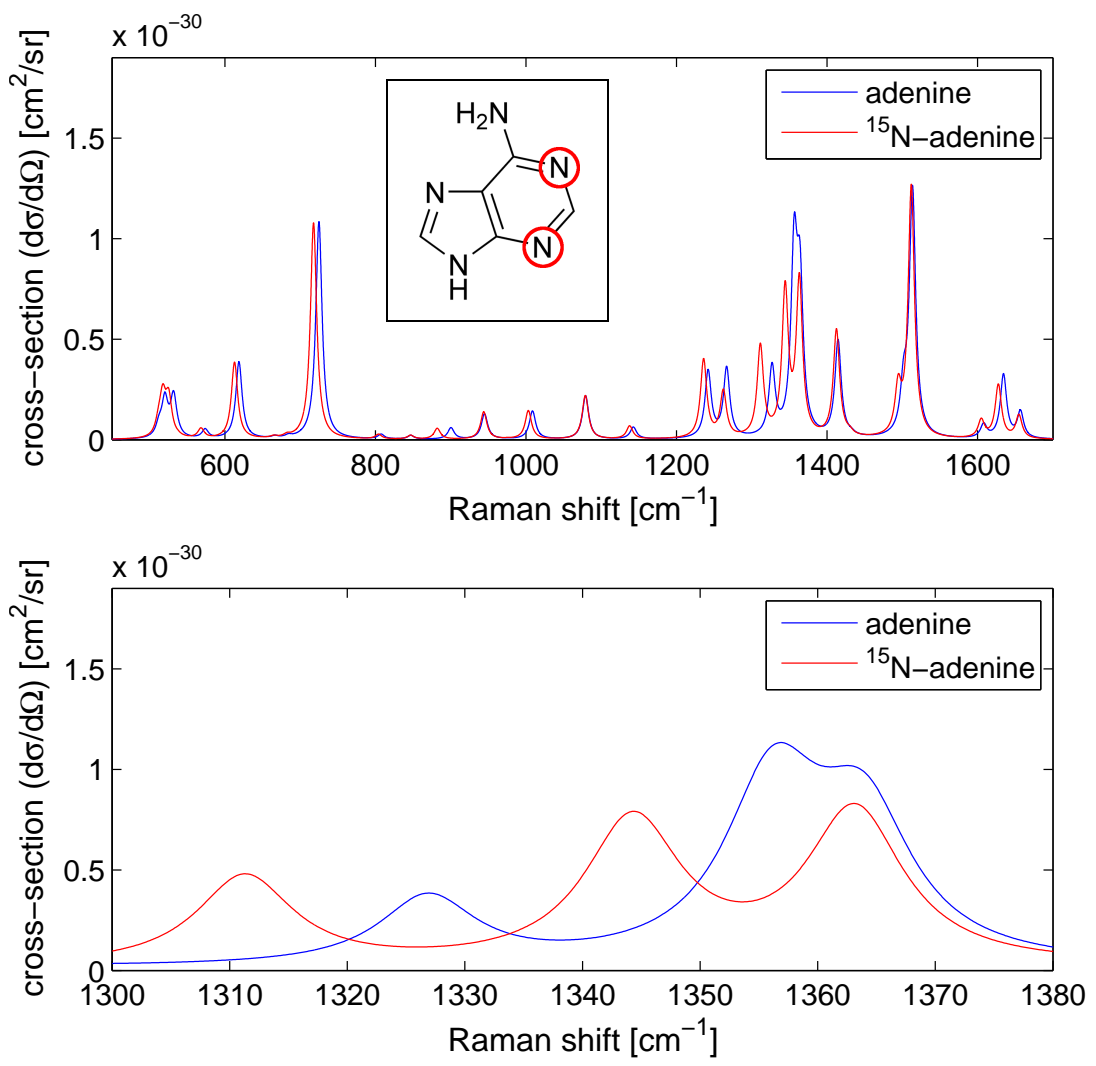

Figure 6.2 - Top plot: simulated Raman spectrum of adenine (blue) and ${ }^{15} \mathrm{~N}$ adenine. The inset shows the Lewis-structure of adenine and highlights which nitrogen atoms are replaced by their isotope. Bottom plot: magnifies into the region in which the most dramatic spectral changes appear.

shifts one should expect to see. From there, one can pick the promising (or commercially available) candidate. Three examples are shown in this context: adenine, imidazole and Rhodamine 800. Figure 6.2 summarises the shifts expected from adenine, assuming isotopic substitution of two nitrogen atoms (see inset of top plot, circled red), ${ }^{14} \mathrm{~N} \rightarrow{ }^{15} \mathrm{~N}$. Many peaks are affected by the substitution (see top plot), however, the strongest spectral differences occur in the region between $1300 \mathrm{~cm}^{-1}$ and $1380 \mathrm{~cm}^{-1}$ (bottom plot). The peaks are shifted (hence easily distinguished), but sufficiently close in energy to avoid background problems if adenine and ${ }^{15} \mathrm{~N}$-adenine were to be used as BiASERS partners. However, adenine is 

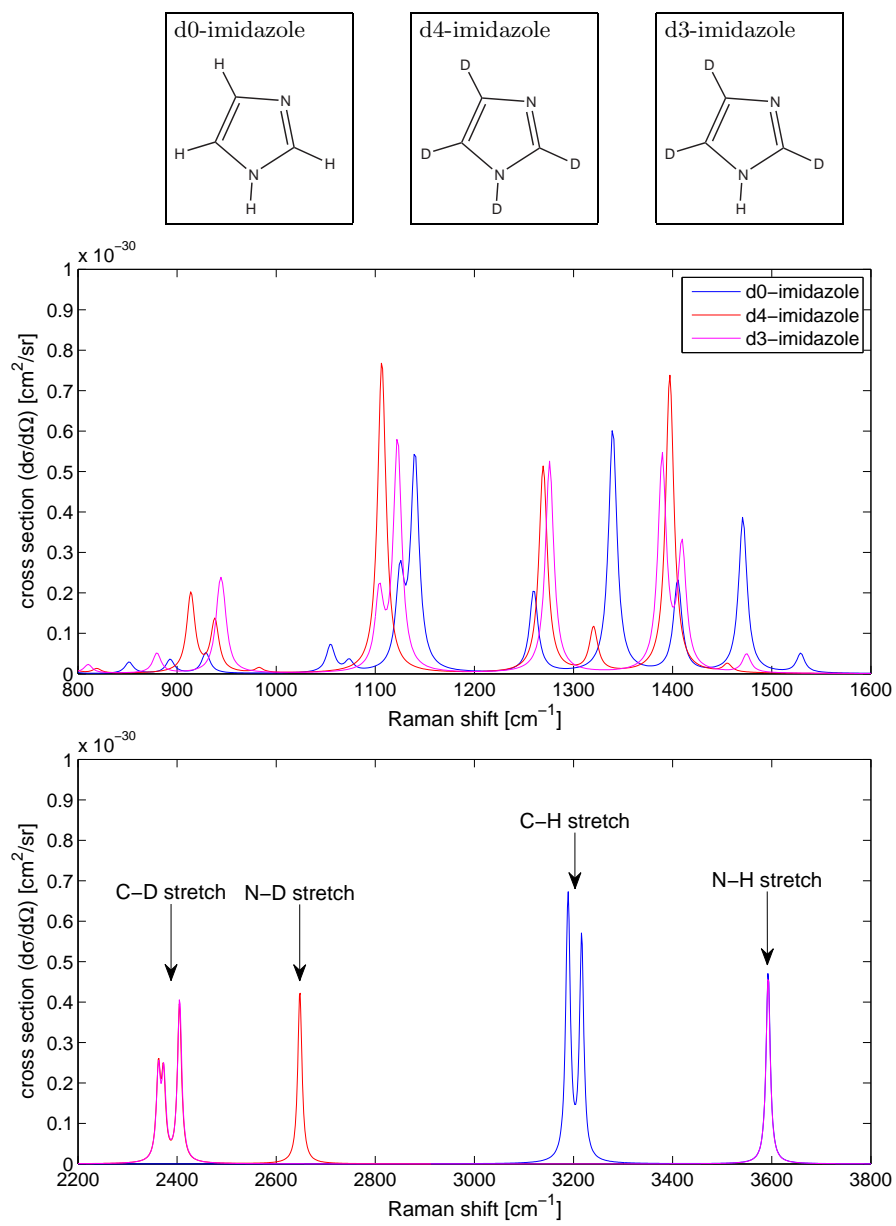

Figure 6.3-Simulated Raman spectra of imidazole. The Lewisstructures of the three calculated isotopic versions of the molecule are shown in the top insets, the Raman spectrum of each is shown in the upper plot. The bottom plot highlights the stretching region, in which distinct spectral changes appear in this case.

a small molecule and is non-resonant in the visible, hence observing SMSERS becomes difficult. To this end, the isotopic shifts have been observed under SERS conditions ${ }^{4}$, but not yet at the single-molecule level.

Along the same lines as for adenine, Figure 6.3 summarises the spectral changes due to isotopic substitution in imidazole. Here, three molecular entities are simulated (see top of figure): d0-imidazole (normal imidazole), d4-imidazole (all hydrogens replaced with deuterium) and the chemically more realistic d3-imidazole (only carbon-bound hydrogens replaced with deuterium). This is a small molecule, hence, again, changes in most peaks are apparent (see top plot). The strongest changes occur in the stretching

\footnotetext{
${ }^{4}$ Unpublished data, courtesy of E. Blackie.
} 


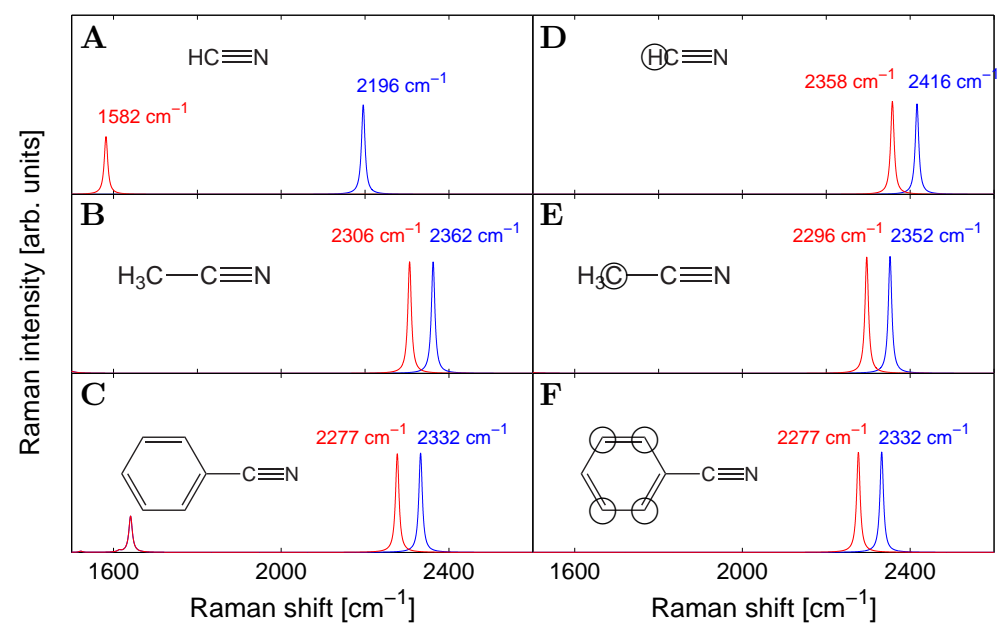

Figure 6.4 - Breakdown of Rh800 for the sake of computational simplicity with respect to the simulation of the cyano group. Shown are molecules and an excerpt of the predicted Raman stretching of the cyano group for ${ }^{12} \mathrm{C}$ (blue) and ${ }^{13} \mathrm{C}$ (red). Plot (A) shows hydrogen cyanide, (B) acetonitrile and (C) cyanobenzene. Plots (D)-(F) pertain to the same molecules, but where atoms are assigned the large mass corresponding to the remainder of Rh800 (circled atoms). Note that already in (D) the predicted isotopic is in good agreement with experiment $\left(\Delta \bar{v}=56 \mathrm{~cm}^{-1}\right)$.

regions (bottom plot). The stretching vibrations are local to the bonds involved (i.e. C-H, etc...) and, therefore, the shifts seen here apply to any molecule featuring these bonds. For example, the C-D stretch in isotopically modified Rhodamine (see below, and also in Sec. 5.3.6) will also occur at $\sim 2400 \mathrm{~cm}^{-1}$.

The final example is Rh800, for which experimental results were discussed in Sec. 5.3.6. The substitution investigated here is the natural isotopic exchange in the cyano group ${ }^{12} \mathrm{C} \equiv{ }^{14} \mathrm{~N}$, where ${ }^{12} \mathrm{C} \rightarrow{ }^{13} \mathrm{C}$ and ${ }^{14} \mathrm{~N} \rightarrow{ }^{15} \mathrm{~N}$. Rh800 is -from the quantum-chemical point of view- a large molecule $\left(\mathrm{C}_{26} \mathrm{H}_{26} \mathrm{~N}_{3} \mathrm{O}\right)$. At the level of theory employed above (method B3LYP, basis set $6-311++G(d, p))$ the calculation of the full molecule becomes a lengthy and impracticable matter. However, the cyano bond, which is at the center of interest here, is a geometrically very localised group in the molecule. As evident from Fig. 6.4, it is possible to extract and simulate only a part 


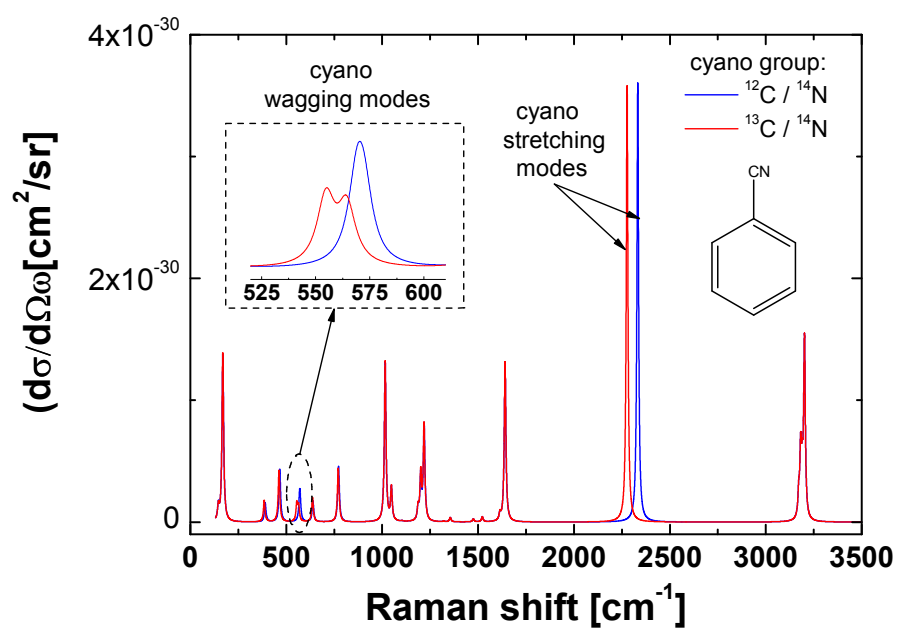

Figure 6.5 - Simulated Raman spectra of cyanobenzene for the isotopic cyano groups ${ }^{12} \mathrm{C} \equiv{ }^{14} \mathrm{~N}$ and ${ }^{13} \mathrm{C} \equiv{ }^{14} \mathrm{~N}$. Note that the prominent shift in the stretching mode as accompanied by a slight shift and splitting of the wagging mode (see inset).

of the full molecule, without sacrificing too much predicitive power. The full molecule (compare Fig. 5.15) is cropped to cyanobenzene (Fig. 6.4 C,F), whereas the carbon atoms at which bonds where cut (circled atoms) are given the unphysical mass which accounts for the remainder of Rh800. This way, the localised vibration of the cyano group 'feels' the rest of the full molecule through the mass of the attached carbons. Taking this approach one step further, cyanobenzene can in principle be cropped to acetonitrile (Fig. 6.4 B,E), and even hydrogen-cyanide (Fig. 6.4 A,D), without sacrificing too much predictive power. This is a property of an isolated vibration, where the corresponding normal mode assigns negligible spatial movement to all atoms except a small local group.

The most prominent isotopic shifts are ${ }^{12} \mathrm{C} \rightarrow{ }^{13} \mathrm{C}$ (since ${ }^{13} \mathrm{C}$ has a natural abundance of $1.1 \%$, as opposed to $0.37 \%$ for ${ }^{15} \mathrm{~N}$. Hence, the Raman spectra of the artificially heavy cyanobenzene were computed assuming the cyano group (i) ${ }^{12} \mathrm{C} \equiv{ }^{14} \mathrm{~N}$ group and (ii) ${ }^{13} \mathrm{C} \equiv{ }^{14} \mathrm{~N}$. These results are shown in Fig. 6.4 and 6.5. The cyano stretching modes appear at $\sim 2300 \mathrm{~cm}^{-1}$, which was confirmed experimentally [149]. Furthermore, an isotopic shift of 
$56 \mathrm{~cm}^{-1}$ is predicted, which is also in good agreement with the experimental spectra (see Sec. 5.3.6 and Ref. [149]). Simulating the influence of ${ }^{15} \mathrm{~N}$, the stretching frequencies (w.r.t. ${ }^{12} \mathrm{C}$ and ${ }^{13} \mathrm{C}$ ) are both predicted to decrease by $\sim 30 \mathrm{~cm}^{-1}$, however, this could not be discerned experimentally. Results pertaining to the observation of isotopically shifted cyano bond frequencies in SM-SERS have been reported in Ref. [149].

\subsection{Obtaining the Raman tensors}

In the case of "Gaussian03" [158] the frequency analysis yields a number of interesting parameters, including (but not limited to) mode frequency, atomic displacement vectors, Raman activity and depolarization ratio. Most important for the endeavor to derive the Raman tensors is the polarisability derivative, which is given at the end of the output file generated by Gaussian. (Unfortunately, to the best of our knowledge, there seems to be no option to print the tensors directly, although all components must be known to the program at some point.) The relevant output thus is, for each mode, the displacement vectors $\vec{r}_{i, j}$ for each $i=\{x, y, z\}$ and each atom $j$, and the corresponding derivatives of the polarisability tensor components, $\frac{\partial \alpha_{\sigma \rho}}{\partial i_{i, j}}$. (The latter is given as one lengthy list of $(6 \times\{x, y, z\} \times$ Number of atoms) numbers in which each derivative tensor is defined by 6 components (the $3 \times 3$ tensors are symmetric), given in the order:

$$
\begin{aligned}
\frac{\partial \alpha_{11}}{\partial \vec{r}_{i, j}}, \frac{\partial \alpha_{12}}{\partial \vec{r}_{i, j}}, \frac{\partial \alpha_{22}}{\partial \vec{r}_{i, j}}, \ldots \\
\frac{\partial \alpha_{13}}{\partial \vec{r}_{i, j}}, \frac{\partial \alpha_{23}}{\partial \vec{r}_{i, j}}, \frac{\partial \alpha_{33}}{\partial \vec{r}_{i, j}}, \ldots
\end{aligned}
$$

The list cycles through all directions $i$ first, before switching to the next atom $j$. Unfortunately, the manual of Gaussian is somewhat sparse in 
documenting this, and it may take a while to work out the correct order, hence the explicit note here.

It should also be noted that the polarisability derivatives $\frac{\partial \alpha_{\sigma \rho}}{\partial \vec{r}_{i, j}}$ and the atomic coordinates (and displacements) are given with respect to two different coordinate systems: the former in internal coordinates used by Gaussian (which in general imposes a cartesian transformation on the original input), the latter in the original frame ${ }^{5}$.

The Raman polarisability tensor of mode $m$ is then simply the displacementweighted sum of the polarisability derivatives:

$$
\hat{R}_{\sigma \rho}^{m}=\sum_{i}^{\{x, y, z\}} \sum_{j}^{\text {atoms }} \frac{\partial \alpha_{\sigma \rho} \rho}{\partial \vec{r}_{i, j}^{m}} \vec{r}_{i, j}^{m} .
$$

To cross-check whether the computed tensor is correct, one can re-compute the ensemble properties, i.e. Raman activity (Eq. 6.5) and depolarisation ratio (see Eq. 6.6), which are part of Gaussians output, and compare the results.

\subsection{Raman tensor visualisation}

Mathematically the Raman tensor is represented by a real, symmetric $3 \times 3$ matrix. While some exposure to and work with tensors can yield intuition and the ability to 'read' a tensors properties, most people will prefer a more visual account of this seemingly abstract object. As will hopefully become clear, judging tensor characteristics like symmetry, orientation and the corresponding selection rules, arguably, becomes easier when tensors are properly visualized. However, one faces a fundamental problem here: a 2nd-rank tensor is a mathematical object which transforms a vector into a new vector with different direction, magnitude. In the case of the Raman tensor, the incident electric field $\vec{E}_{L}$ is turned into the Raman dipole:

\footnotetext{
${ }^{5}$ Using the keyword "NoSymm" avoids reorientation of the molecule.
} 
$\vec{p}_{R}=\hat{R} \vec{E}_{L}$. Hence, a bundle of vectors defined in $3 \mathrm{~d}$ space is turned into a different bundle. This poses a fundamental dilemma since there is (obviously) no concise and convenient method of plotting a vector-valued function defined over a vector field. Therefore, tensor visualisation must compromise on some level; leaving the question: which plotting approach emphasizes crucial properties (Raman intensity, selection rules) most?

In the following, a few options are explored and their respective advantages and shortcomings are pointed out.

\section{Classical approaches}

A classic strategy is to visualise the tensor directly by eigen-transforming it into diagonal form:

$$
\begin{aligned}
& \hat{R} \vec{v}_{i}=\lambda_{i} \vec{v}_{i} \\
& \Rightarrow \quad\left(\begin{array}{lll}
\vec{v}_{a} & \vec{v}_{b} & \vec{v}_{c}
\end{array}\right)^{T} \hat{R}\left(\begin{array}{lll}
\vec{v}_{a} & \vec{v}_{b} & \vec{v}_{c}
\end{array}\right)=\left(\begin{array}{lll}
a & 0 & 0 \\
0 & b & 0 \\
0 & 0 & c
\end{array}\right),
\end{aligned}
$$

whereas the three orthogonal eigenvectors $\vec{v}_{a}, \vec{v}_{b}, \vec{v}_{c}$ are denoted as principal axes and the associated eigenvalues $a, b, c$ define the tensor's extend along its principle axes. The tensor is then visualized as an ellipsoid (see Fig. 6.6).

This straight-forward approach seems -at first sight- powerful and sufficient, one gets a clear idea of the tensors general direction or isotropy (or partial isotropy, i.e. oblate or prolate tensors) with respect to the molecular frame to which it is attached. However, information on the direction of the vector resulting from the tensor operation is lost. For example, consider the following two tensors:

$$
\hat{R}_{a}=\left(\begin{array}{ccc}
a & 0 & 0 \\
0 & b & 0 \\
0 & 0 & c
\end{array}\right), \quad \hat{R}_{b}=\left(\begin{array}{ccc}
-a & 0 & 0 \\
0 & b & 0 \\
0 & 0 & c
\end{array}\right) .
$$



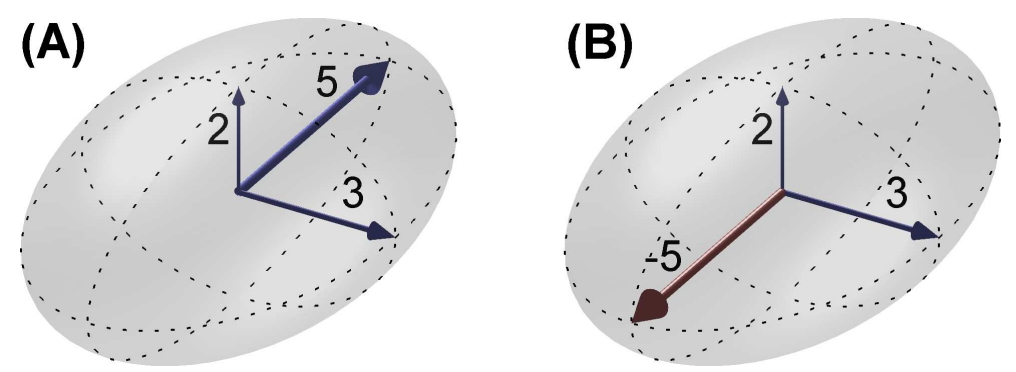

Figure 6.6 - Ellipsoid-based visualization of two 2nd-rank tensors. Drawn are the semi-principle axes (arrows) and the equators (dotted lines) for each axis. The principle axes are the eigenvectors of the tensor; the eigenvalues are the semiaxes, i.e. the tensor's extend along the principle axes. Both tensors shown here are scalene, i.e. $|a|>|b|>|c|$ (see Eq: 6.10). Note how a flip in sign of an eigenvalue leads to the same ellipsoid.

Both, $\hat{R}_{a}$ and $\hat{R}_{b}$, lead to the same ellipsoid (see Fig. 6.7a and 6.7b). This does not properly portrait the physical reality; the resulting Raman dipoles as well as the angular intensity distributions of light scattered by $\hat{R}_{a}$ and $\hat{R}_{b}$ are by no means the same.

Another conceivable approach takes account of the vectorial character of the transformation. Here, for convenience, Eq. 2.1 is re-cast as $\vec{e}_{p}=\hat{R} \vec{e}_{L}$, where the field $\vec{E}_{L}$ and the Raman dipole $\vec{p}$ appear simply as dimensionless, normalised directions, $\vec{e}_{L}$ and $\vec{e}_{p}$. Now one can plot the difference vectors $\vec{e}_{p}-\vec{e}_{L}$, which, combined with the previous approach, relieves its ambiguity. However, as Fig. 6.7c and 6.7d show, this is not very intuitive and can become fairly obscure already in two dimensions. The inherent problems in visualizing such vector fields are easily overcome in the SERS/TERS regime.

\section{Surface-enhanced regime}

Under SERS/TERS conditions a reasonable visualization method can be pursued which is based on the only measurable quantity: Raman intensity. In essence, the tensor transformation is reduced to a scalar-valued function 
(a)

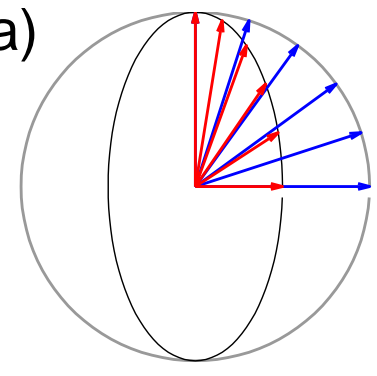

(c)

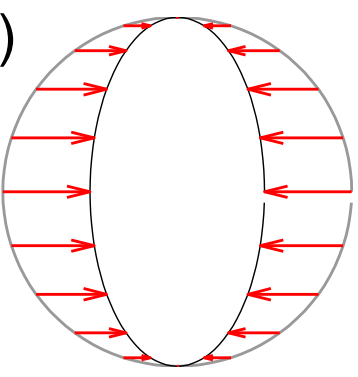

(e)

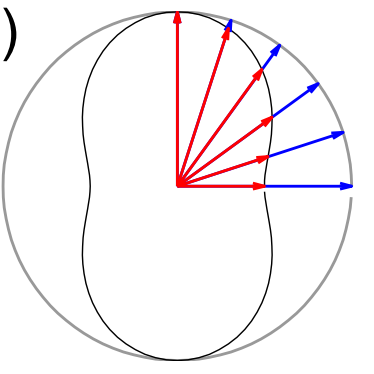

(b)

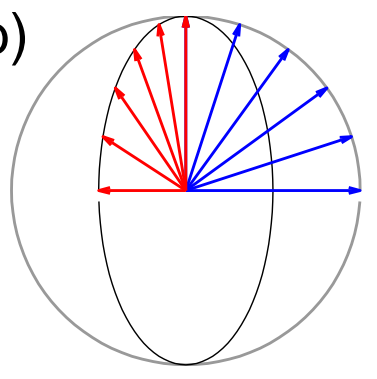

(d)

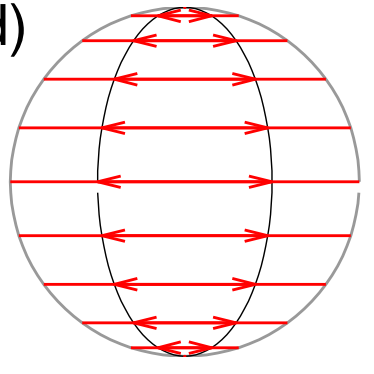

(f)

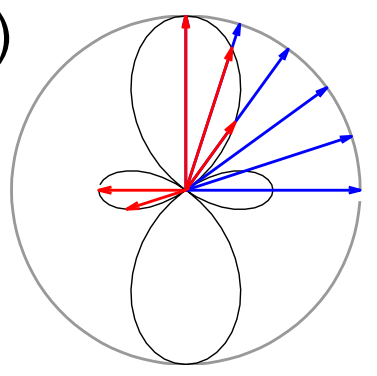

Figure 6.7 - Comparison of visualizations for tensors $\hat{R}_{a}$ and $\hat{R}_{b}$ (see Eq. 6.11). For clarity the plots are restricted to the $x-y$ plane. (a) shows all possible incident polarizations $\vec{e}_{L}$ (grey circle, this arrows) and the normalized ellipsoid obtained from the resulting Raman dipoles $\vec{p}_{R}=\hat{R} \vec{e}_{L}$ (black ellipse, thick arrows). (b) is the same as in (a), but for tensor $\hat{R}_{b}$. Note that one cannot distinguish the tensors by their ellipsoids; only by explicitly drawing the dipole moments the difference is highlighted. (c) and (d) show the vector differences $\vec{p}_{R}-\vec{e}_{L}$ for $\hat{R}_{a}$ and $\hat{R}_{b}$. In (e) and $(\mathrm{f})$ the angular dependence of the intensity obtained by parallel contraction of the tensor, $\left(\vec{e}_{L} \hat{R}_{a} \vec{e}_{L}\right) \vec{e}_{L}$, in is plotted. Note that in (f) it becomes apparent that $\hat{R}_{b}$, for some excitation/detection polarizations, yields no Raman intensity. See text for details. 
defined over a vector field.

First, it is instructive to note that the intensity is a scalar function proportional to the projection of the Raman dipole $\hat{R} \vec{e}_{L}$ onto the detection polarization $\vec{e}_{D}$, thus:

$$
I \propto\left|\vec{e}_{D}^{\mathrm{T}} \hat{R} \vec{e}_{L}\right|^{2},
$$

This is still a quantity dependent on two separate vectors in $3 \mathrm{D}$ space, $I=f\left(\vec{e}_{D}, \vec{e}_{L}\right)$. This is, however, only strictly true in the general case of a classical Raman scattering experiment; conversely, under SERS conditions, one can exploit the uniaxiality of the plasmon-driven hot-spots.

As elaborated upon in chapter 3, the local field orientation is largely independent of the specific incident field polarisation. The local field governs both, the excitation of the Raman dipole, as well as the re-radiation of the scattered light. Consequently, under SERS conditions, the incident polarization felt by the molecule is that of the local field, $\vec{e}_{\text {local }}$, and, moreover, the detection polarization is intrinsically fixed to the very same vector. One basically excites and detects through the same nanoscopic polarizer: the SERS hot-spot. Depolarization ratios of non-resonant molecules (e.g. benzenethiol) under SERS conditions have been shown to be $1 / 3$ independent of analyte and normal mode ${ }^{6}$, confirming the overriding uniaxial response of SERS hot-spots. In conclusion, it holds that $\vec{e}_{D} \| \vec{e}_{L}$, and thus it is reasonable to consider the parallel tensor contraction:

$$
I_{\|}=f\left(\vec{e}_{\text {local }}\right) \propto\left|\vec{e}_{\text {local }}^{\mathrm{T}} \hat{R} \vec{e}_{\text {local }}\right|^{2} .
$$

Without losing generality, one can restrict the field vectors $\vec{e}_{\text {local }}$ to point onto a unit sphere. Then, the resulting surface $I_{\|}$can be plotted, since it depends only on one set of spatial coordinates. This is done for the 2D case in Fig. 6.7e and 6.7f. Note that this visualization highlights the difference in selection rules - whereas tensor $\hat{R}_{a}$ (see Eq. 6.11) will be Raman active for any orientation of $\vec{e}_{\text {local }}$, tensor $\hat{R}_{b}$ shows zero intensity for angles:

$$
\varphi=\arccos \left(\sqrt{\frac{b}{b-a}}\right),
$$

\footnotetext{
${ }^{6}$ See Fig. 3 in Ref. [72]
} 
(in the x-y plane), and when $\vec{e}_{\text {local }}$ is perpendicular to the $\mathrm{x}-\mathrm{y}$ plane (not shown).

On the one hand, plotting the intensity resultant from a parallel tensor contraction, $I_{\|}$, makes sense only in the context of SERS/TERS and has no apparent applicability under classical Raman conditions. On the other hand, only under SERS conditions it makes sense to look at the spatial details of the tensors in the first place since (i) only in SERS one can observe single/few molecules instead of large ensembles and (ii) the effects for which detailed knowledge of the tensors is important are the surface selection rules, i.e. a pure SERS effect. Hence, this type of visualization will be used in the following.

\subsection{Raman tensors of benzotriazole}

Visualising parallel contracted tensors, as described above, provides sufficient convenience to now apply this idea to the DFT-simulated Raman tensors of an example molecule, benzenethiol, and compare these findings to what is observed experimentally. To this end, the DFT analysis is based on the isolated, free-space molecule, which obviously is of limited comparability to the experimental SERS data (these shortcomings will be addressed specifically further below). The applicability of this approach in general needs to be judged on a case-by-case basis, since, ultimately, it is the agreement or disagreement with experiment which validates or invalidates the correctness of the DFT predictions. In some cases it may help understanding experiment (as, for example, in the identification of the binding site of benzenethiol, see Sec. 6.4), in other cases it will simply highlight the limitations of the approach, thereby pointing out where higher levels of sophistication are needed. Hence, in the following, DFT Raman tensors shall be treated as a zero-order approximation to the real tensors under SERS conditions.

In general, since $I_{\|}$is a surface in 3D space, it requires 2D-plots from dif- 
ferent viewpoints. For each tensor, three plots $(x-y, x-z, y-z)$ will minimize visual ambiguity. In Figures 6.8, 6.9 and 6.10 the following is shown:

- (top right corner) a ball-stick representation of the optimized molecular geometry with displacement vectors (red arrows) of the respective mode. Here the coordinate frame is omitted; it should be noted that benzotriazole is a planar molecule, and the molecular plane is oriented so it coincides with the $x-y$ plane. Accordingly, this plot uses the same viewpoint as the plot to its left. Hence, the molecule is shown with respect to the same coordinate system as its tensors which is important in this context since the tensors are intrinsically linked to the molecular orientation. To retain clarity, all modes plotted are in-plane modes, i.e. the vibrational atomic displacements occur only in the molecular plane.

- (top left corner) projection of the Raman parallel intensity $I_{\|}$(see Eq. 6.13) into the $x-y$ plane,

- (lower left corner) x-z projection of $I_{\|,}$

- (lower right corner) y-z projection of $I_{\|}$.

As the reader will notice, for the normal modes shown here $\left(1014 \mathrm{~cm}^{-1}\right.$, $\left.1045 \mathrm{~cm}^{-1}, 1625 \mathrm{~cm}^{-1}\right)$, most of the interesting angular dependence of the Raman tensors happens to lie in the molecular plane, i.e. the $x-y$ plane. This comes as no surprise since, as stated earlier, these exemplary modes are in-plane vibrations. In-plane modes are the predominant Raman peaks observed for planar organic molecules. This yields the convenience, as apparent in Figs. 6.8, 6.9 and 6.8, that one can restrict the plots to the $x-y$ projection and, thereby, represent the tensorial characteristics in a single plot.

The normal modes at 1014, 1045 and $1625 \mathrm{~cm}^{-1}$ and their respective Raman tensors have some exemplary properties: 


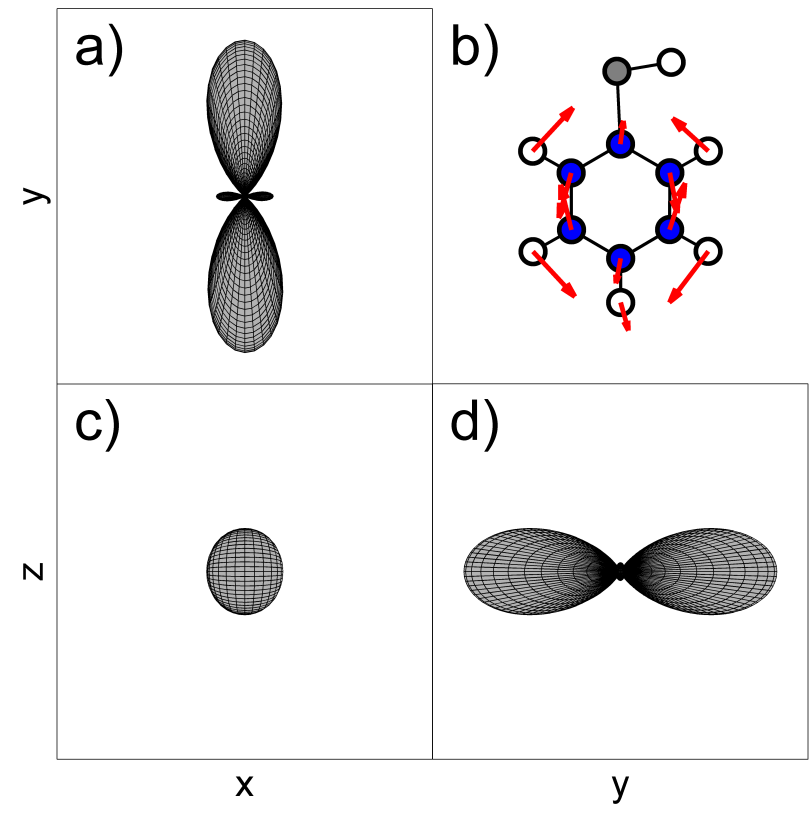

Figure 6.8 - Normal mode and Raman tensor representation of the vibrational mode of benzenethiol at $1625 \mathrm{~cm}^{-1}$. Plot (a) shows the projection of the tensor's response $\left|\vec{e}_{\text {local }}^{T} \hat{R} \vec{e}_{\text {local }}\right|^{2}$ into the $x-y$ plane, (b) plots the molecule with displacement pattern of the normal mode, projected into the $x$-y plane as well. (c) and (d) plot the tensor's response in the $x-z$ and $y-z$ plane, respectively. All plots are drawn with respect to a common coordinate system.

- Fig. 6.8 , i.e. the normal mode at $1625 \mathrm{~cm}^{-1}$, is a near-uniaxial tensor. It is aligned with the y-axis (the molecular long axis) and closely resembles a textbook example of the $\cos ^{2}$ distribution (which is often presented when vectors get projected onto a fixed direction, for example in the interaction of a polarized electrical field with a fluorescence dipole). The tensor also has a fairly minor eigenvalue along the $x$-axis, which differs in sign from the dominant eigenvalue, and hence leads to two additional, small lobes in the projection (compare to Fig. 6.7e-f). In this coordinate system the tensor is a near diagonal matrix (for reference, see Eq. 6.10) with its main component along the y-axis, thus: $|b| \gg|a| \cong|c|$.

- Fig. 6.9, i.e. the normal mode at $1045 \mathrm{~cm}^{-1}$, is a partially isotropic tensor 


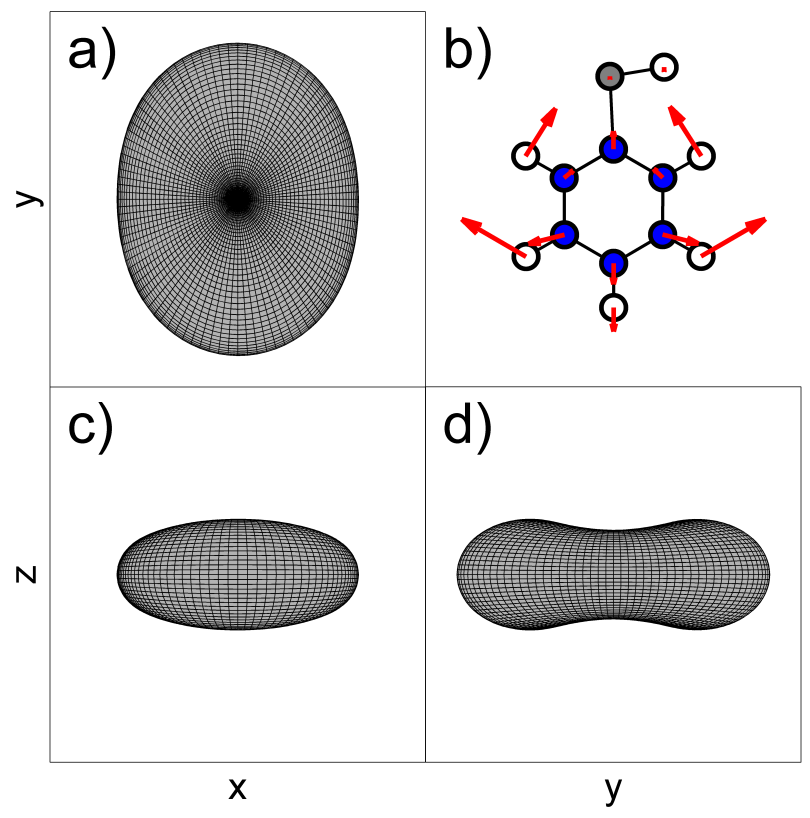

Figure 6.9 - Normal mode and Raman tensor representation of the vibrational mode of benzenethiol at $1045 \mathrm{~cm}^{-1}$. Plots as in Fig. 6.8 .

in the $x-y$ plane. It closely resembles an oblate ellipsoid, and in its diagonal form one finds components $|a| \approx|b| \gg|c|$. (A fully isotropic tensor would feature $a=b=c$ and plot here as a sphere; for example, the fully symmetric hydrogen stretching of $\mathrm{CH}_{4}$ at $2917 \mathrm{~cm}^{-1}$ is of this type.) The key difference to the previously discussed uniaxial tensor is that while the Raman intensity has a strong angular dependence in the uniaxial case, an isotropic tensor will yield the same Raman intensity irrespective of the electric field orientation (as long as it is inplane, for this case). Therefore a normal mode with an uniaxial tensor strongly depends on the orientation of the molecule with respect to the electric field; while a fully isotropic normal mode will not vary at all with the molecular orientation.

- Fig. 6.10, i.e. normal mode at $1014 \mathrm{~cm}^{-1}$, is a mode of mixed character: the tensor is neither fully uniaxial nor isotropic (or oblate). The major principal axis lies roughly parallel to the y-axis (along the long axis of the molecule), however, excitation along $\mathrm{x}$ will still produce about 


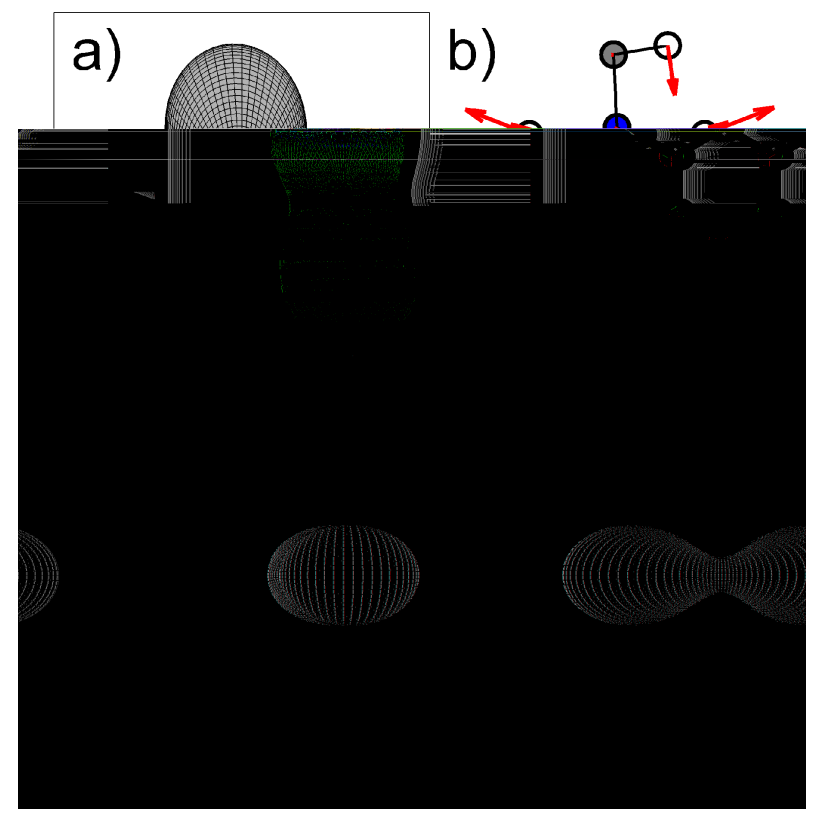

Figure 6.10 - Normal mode and Raman tensor representation of the vibrational mode of benzenethiol at $1014 \mathrm{~cm}^{-1}$. Plots as in Fig. 6.8.

$1 / 3$ of the maximal intensity. The eigenvalues of this tensor are equal in sign, hence resulting in the node-free projection.

Figure 6.11 summarizes the static-field DFT results for seven prominent in-plane modes. The top row in the figure shows the pattern of atomic displacements for each normal mode; the second row plots the tensor projections corresponding to these modes in the $x-y$ plane (same coordinate system as the molecule drawn above). The complete Raman spectrum, as a result of the DFT calculation, is plotted by assuming Lorentzian peak shape with a uniform width of $10 \mathrm{~cm}^{-1}$. Measured normal Raman and SERS spectrum are shown as well (offset for clarity) and indicate a good agreement of the simulation with the experiment. Note that the tensors of the modes shown here are either uniaxial or (partially) planar isotropic, with the exception of the slight bi-axiality of the mode at $1625 \mathrm{~cm}^{-1}$.

With the experiment in mind, what can be learned from these tensor visualisations? The local field in SERS is fixed in its polarisation with respect 


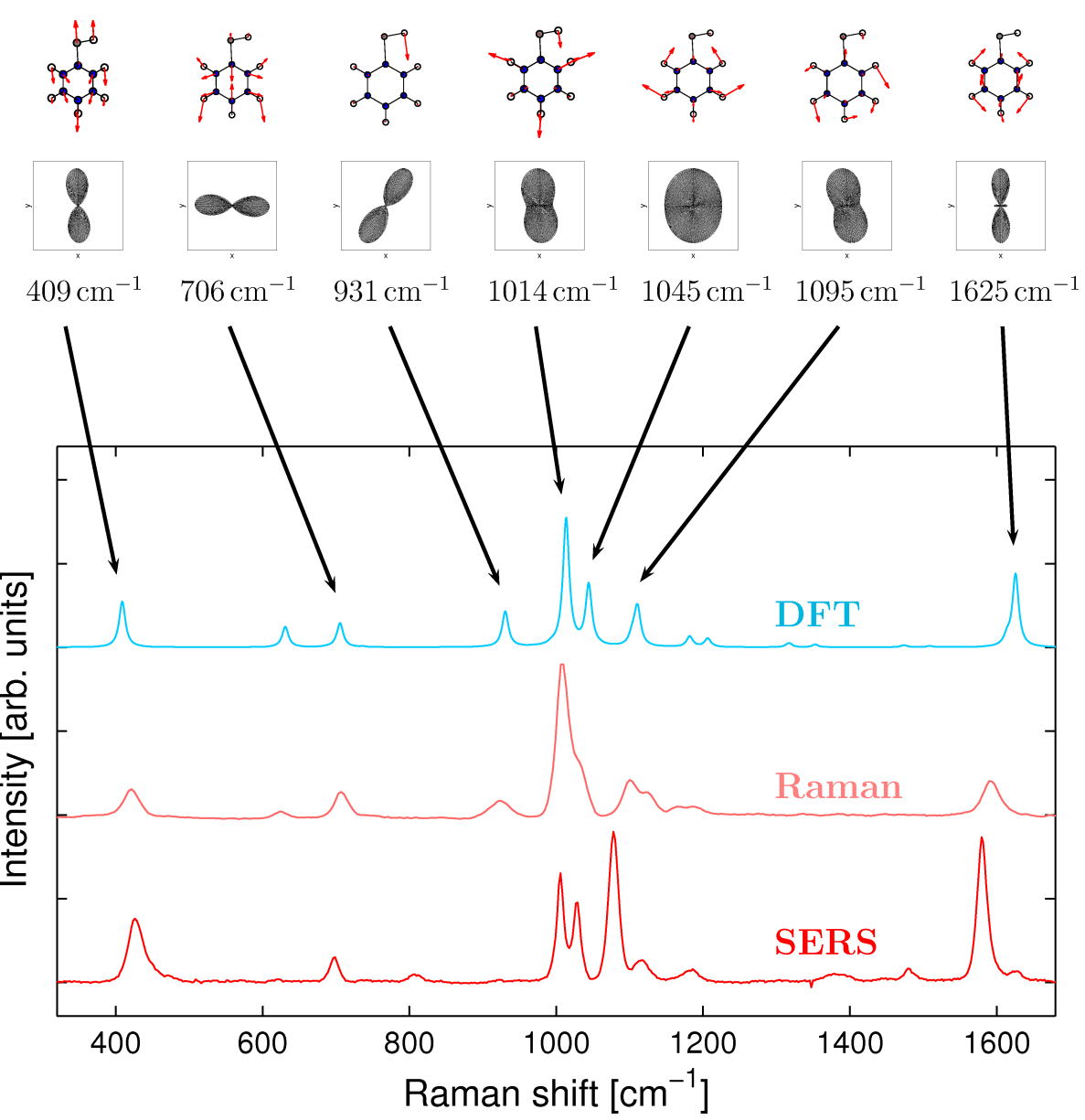

Figure 6.11 - Static-field DFT results with experimental context. Top: displacement vectors, static-field Raman tensors and Raman shift of seven normal modes of benzenethiol (molecule and tensors drawn with respect to the same coordinate system). Bottom: Full static-field DFT Raman spectrum (rendering each peak as Lorentzian with width $10 \mathrm{~cm}^{-1}$ ), experimental Raman spectrum of benzenethiol in water and SERS of benzenethiol on Ag-colloids in water, both at off-resonance excitation $(633 \mathrm{~nm})$. 
to the surface. By calculating the Raman intensity due to a fixed field for all modes, one gets the relative intensities the Raman modes have with respect to each other. This comes with caveats, but can -in principle- help to deduce the molecular orientation on the surface, as shall be described in the following.

\subsection{Connection to molecular orientation}

In SERS conditions, molecules are usually chemisorbed or physisorbed to the surface; the former meaning the formation of a chemical bond between analyte and surface, the latter indicating a somewhat looser association (e.g. Rh6G is thought to physisorb to Ag colloids, since it the molecule is electrostatically attracted to the surface, but does not appear to form a bond). The chemical binding of the molecule to the surface restricts the molecule's degrees of freedom, that is, surface-bound molecules have an average orientation due to the partial restriction in their rotation. This may also be true to some extend in the case of a physisorbed molecule.

In principle, with the tensors and their principal axes and shapes (uniaxial, oblate isotropic, etc..,) known, it becomes possible to predict the fluctuations in the relative intensity of modes. In Fig. 6.11, note the tensors of the modes at $409 \mathrm{~cm}^{-1}$ and $1625 \mathrm{~cm}^{-1}$; both are uniaxial with parallel principal axes. Consider applying a range of different incident field polarizations to a fixed molecule and monitor the intensity of these two modes: the observed intensities of these modes would correlate. The contrary is to be expected from the pair $409 \mathrm{~cm}^{-1}$ and $706 \mathrm{~cm}^{-1}$ : as the field orientation changes from the $y$-axis to the $\mathrm{x}$-axis, one mode will decline in intensity while the other rises, and vice-versa, resulting in anti-correlation. Logically, the $1625 \mathrm{~cm}^{-1}$ mode would also anti-correlate with the mode at $706 \mathrm{~cm}^{-1}$. By the same token, little or no correlation at all is expected when comparing the mode at $1045 \mathrm{~cm}^{-1}$ to any other mode, due to the isotropy of this mode.

Fig. 6.12 shows the results of this analysis. On the left, the orientation of 
benzenethiol (A) with respect to the simulated local field direction (B) is shown, as it was used for the generation of theoretical mode intensities. To induce fluctuations, the field direction was varied uniformly within a cone of $\sim 15 \mathrm{deg}$ around the direction indicated. On the right, the correlation of peaks in their intensity fluctuations is plotted as a colour-map. Note, for example, how the $409 \mathrm{~cm}^{-1}$ mode anti-correlates with the $631 \mathrm{~cm}^{-1}$ and $706 \mathrm{~cm}^{-1}$ modes, but correlates with other modes (compare Fig. 6.11, see also Fig. 6.14 for the tensor of the $631 \mathrm{~cm}^{-1}$ mode).

To recap the connection to the experimental situation: in a SERS hot-spot the local field polarisation is fixed, but surface-bound molecules rotate according to the degrees of freedom whose limits are imposed by the bond and the surface. Hence, unless the molecular orientation is completely fixed, one can expect a molecule in a hot-spot to map out at least part of the expected (anti-)correlations of the relative mode intensities. In principle, one could perform the correlation analysis on experimental data, and then modify the molecule's simulated orientation with respect to the local field in such a way that one obtains the same correlation coefficients. In other words, one could exploit the detailed knowledge of DFT tensors to derive the average molecular orientation and even the molecules' degrees of freedom, however, in the real world, a number of problems inhibit this approach:

- An intrinsic flaw to the analysis outlined above is that Raman tensors are modified due to the presence of the surface. The free-space tensors are in general not the same as the Raman tensors of the analyte-surface complex. Attempts to model the entire surface complex via DFT are -to date- computationally very expensive and suffer from basis sets and DFT methods that are inaccurate in modeling the electronic structure of the involved metal atoms.

- The enhancement of a SERS hot-spot is not spectrally flat, therefore Raman modes are altered in their relative intensities due to their mismatch with the plasmon resonance of the hot-spot. This is schematically shown in Fig. 6.13. SERS hot-spots which show enhancements 
A

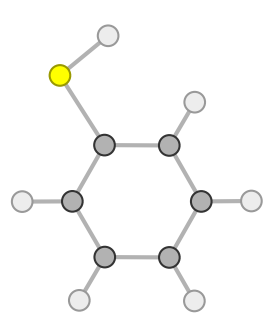

B

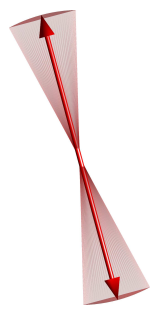

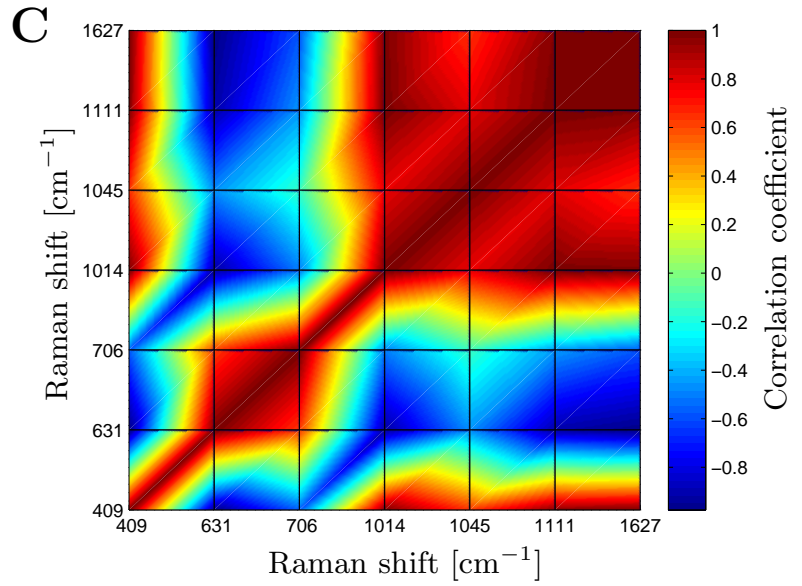

Figure 6.12-(A) Structure of benzenethiol with (B) indicated local field direction. (C) Cross-correlation map of the peak intensity fluctuations of selected modes. See text for details.

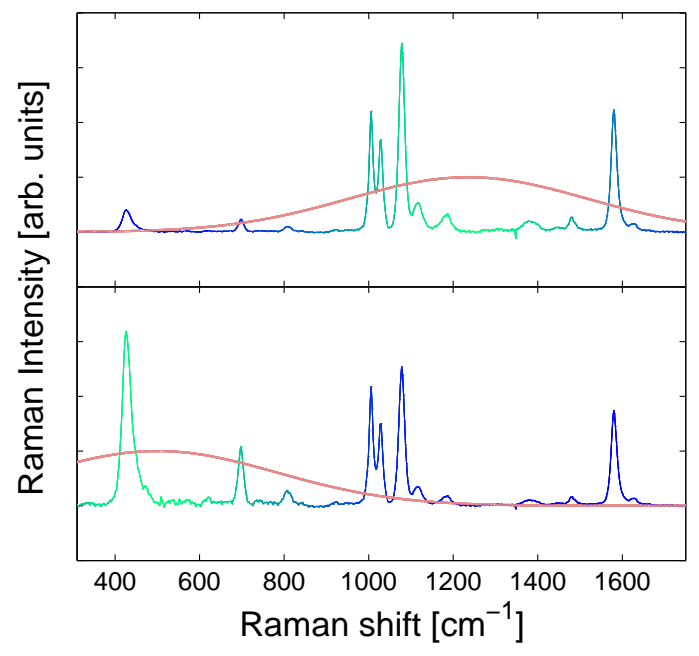

Figure 6.13 - Schematic of the influence of plasmon dispersion on the relative peak intensity, with the plasmon resonance (red line) at $\sim 1200 \mathrm{~cm}^{-1}$ (top plot) and at $\sim 400 \mathrm{~cm}^{-1}$ (bottom plot). 
large enough to observe single molecules are sensitive in their spectral resonance to local geometry changes on the nanoscale (e.g. varying the gap between two colloids by $\pm 1 \mathrm{~nm}$ ). This is apparent and well known from the fluctuations $[144,163]$ in the background spectrum (which is believed to be modified fluorescence [68, 164]) observed in many SERS experiments. The fluctuations due to plasmonic dispersion largely outweigh the orientation induced fluctuations.

These are two problems intrinsic to the theoretical and experimental approach, and are hard to overcome. Another, less intrinsic, but noteworthy problem lies in the photobleaching of the analyte and photochemistry on the substrate. This will not necessarily affect relative peak intensities, but it further inhibits reproducibility on the nanoscale level.

Single molecules detection using SERS thus is a sword with at least twoedges: on the one hand, it simplifies the tensor representation and projects out selection rules through the fixed nature of the local field; on the other hand it complicates the matter vastly through tensor and intensity modifications. An additional problem in SM-SERS lies in its exploitation of the additional enhancement from the electronic resonance of the molecule, whose effect on the Raman tensors will be touched in the next section.

\subsection{Moving towards resonance}

Ultimately, as pointed out earlier, the full advantage of the spatial Raman tensor analysis and representation comes to play in the single molecule regime, since any ensemble will potentially result in orientational averaging, unless all ensemble constituents are in the exact same orientation (e.g. in ordered aggregates).

Roughly ten years agoSERS was suspected $[8,9,12]$ and recently proven [14, $127,145]$ to provide single-molecule (SM) sensitivity (see chapter 5$)$. However, this has so far only been achieved by combining the electromagnetic 
enhancement with resonant Raman scattering, i.e. in the regime of surfaceenhanced resonant Raman scattering (SERRS).

One naturally arrives at the questions: Are the off-resonance Raman tensors retained when the molecule is driven into its electronic resonance? By how much do the Raman tensors change, and what are the mechanisms that drive such a transformation? These questions have received much consideration far before the advent of SM-SERS, and the principle mechanisms (e.g. Franck-Condon factors, Dushinsky effect) are known (for reviews, see Refs. [165, 166, 167]). I do not attempt to showcase groundbreaking new quantum theoretical results here, instead, this section sets out to highlight some fundamental properties of resonant Raman tensors, while being aware of the problems intrinsic to the method of prediction. For this, coupled-perturbed Hartree-Fock (CPHF) calculations were conducted - at this level of theory, the Hamiltonian is modified via perturbation theory to include the effect of an oscillating electrical field. This gives the opportunity to vary the excitation wavelength, from a static field $\left(v_{L}=\infty\right)$ to the resonance in the UV. The electronic wavefunction is calculated for a perturbed Hamiltonian, i.e. the coupling into unoccupied states is implicit, but there is no accounting for the Franck-Condon overlaps or mode-mixing via excited states (Dushinsky effect [168]).

Therefore, the implementation of the electric field perturbation in the CPHF equations in Gaussian03 [158] strictly only allows for the calculation of pre-resonance Raman polarisabilities, i.e. for as long as vibronic effects are negligible. To find out where the resonance edge is in the theoretical molecule, time-dependent DFT (TDDFT) calculations were carried out (method B3LYP, basis 6-311++G(d,p)), which yield the electronic transition energies and dipole moments.

Table 6.2 shows, as resulting from the TDDFT calculation, the first (i.e. energetically lowest) transitions. Shown are the transition wavelengths, oscillator strengths and depictions of the transition dipoles. Note that the transition dipole of the second transition is aligned with the z-axis, i.e. 


\begin{tabular}{llll}
$\begin{array}{l}\text { transition } \\
\text { number }\end{array}$ & $\begin{array}{l}\text { wavelength } \\
{[\mathrm{nm}]}\end{array}$ & $\begin{array}{l}\text { oscillator } \\
\text { strength }\end{array}$ & $\begin{array}{l}\text { transition } \\
\text { dipole }\end{array}$ \\
\hline 1 & 262 & 0.0134 & 0.0026 \\
2 & 261 & & \\
3 & 240 & 0.2165 & \\
\hline
\end{tabular}

Table 6.2 - Parameters of the first three transitions of benzenethiol calculated via TDDFT.

perpendicular to the molecular plane. Clearly, the third transition is the strongest here, whereas the second is negligibly weak.

Aware of the resonant transitions, the Raman tensors are calculated using the CPHF method, starting with an excitation far from resonance and gradually moving towards it. The results of this calculation are shown in Fig. 6.14. As before, the projection of selected modes into the $x-y$ plane is plotted. In each column, the tensor projections of one mode are shown for 11 different excitation wavelengths $\lambda_{L}$, from static field $\left(\lambda_{L}=\infty\right.$, top row) up to the third resonance transition $\left(\lambda_{L}=240 \mathrm{~nm}\right.$, bottom row). The top row of tensors, at static excitation field, are the same as those shown earlier, see Fig. 6.11. A number of observation can be made in this figure:

- Approaching resonance, a manifold of tensor transformations can be observed. A drastic example is the $409 \mathrm{~cm}^{-1}$ mode, which keeps its uniaxial character (aligned along the y-axis) until $350 \mathrm{~nm}$ excitation; however, at $330 \mathrm{~nm}$, the mode has transformed into a tensor with an axial component along $\mathrm{x}$, and isotropic shape in the $\mathrm{y}-\mathrm{z}$ plane. This changes into an in-plane (semi-)isotropic tensor for $300 \mathrm{~nm}$ and $280 \mathrm{~nm}$. 


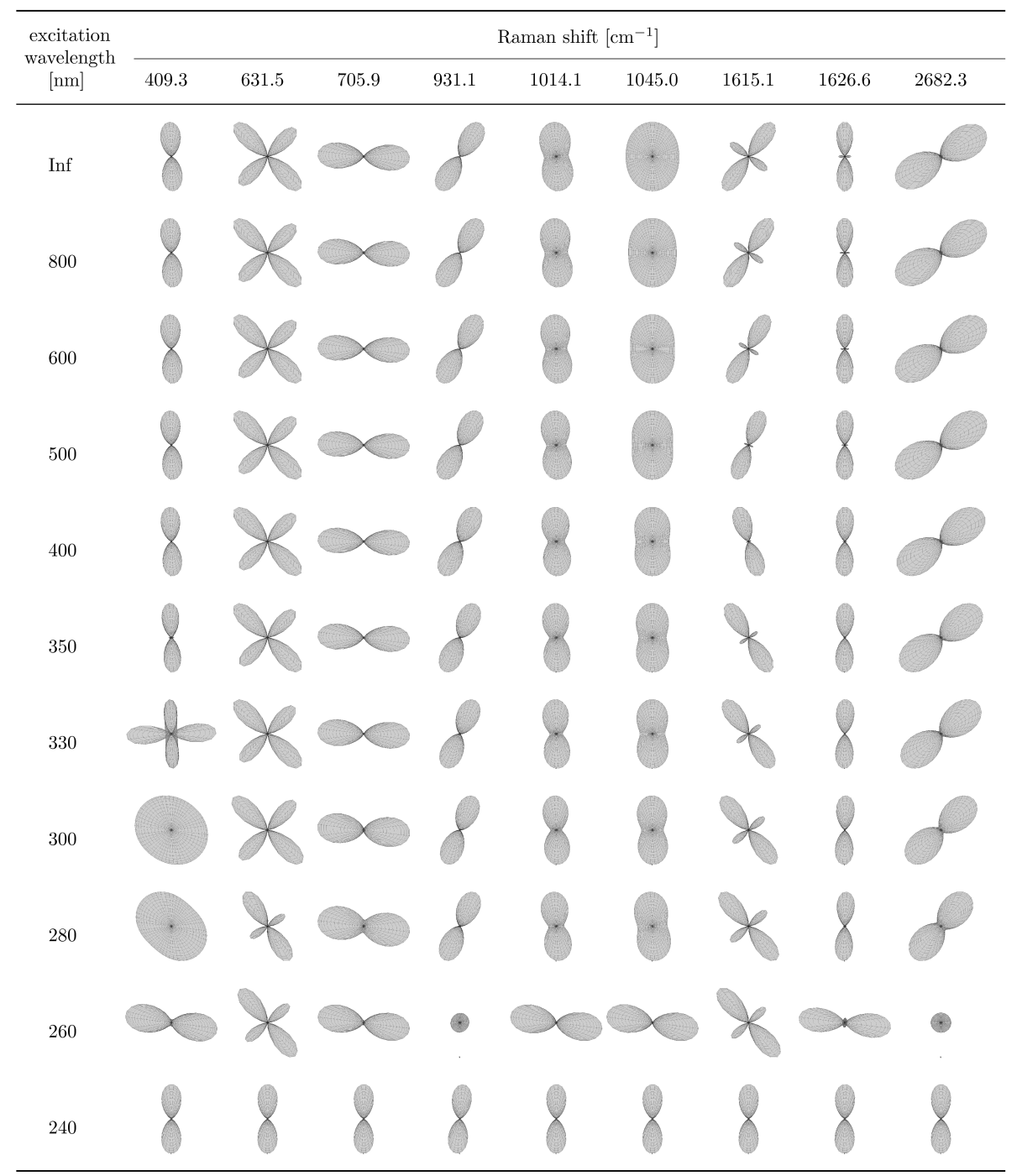

Figure 6.14 - Excitation wavelength dependence of the Raman tensors of nine selected normal modes of benzenethiol, as obtained by CPHF calculations. Columns refer to the same mode, rows refer to the same excitation wavelength. The tensors are projected in the $x-y$ plane of the same coordinate system as used in the previous figures (compare Fig. 6.11). See text for details. 
- In pre-resonance, the other modes also undergo changes in their respective principal axes, and, to a lesser degree, in their shape.

- Most strikingly, however, is that in resonance the uniaxial tensor character is prevalent (most modes in the $260 \mathrm{~nm}$ resonance, all modes in the $240 \mathrm{~nm}$ resonance). Moreover, the principal axes of the tensors are aligned to the respective transition dipoles (compare Table 6.2).

- For some modes the tensor symmetries and orientations have already undergone substantial change at excitation wavelengths far from resonance. Examples are the $409 \mathrm{~cm}^{-1}$ mode mentioned above and the mode at $1615 \mathrm{~cm}^{-1}$. This indicates that a pre-resonance effect can modify the Raman cross-section, which has impact on the detection limit in SERS (for most modes, the cross-section increases in pre-resonance; see below) and, in particular, it has impact on the accurate determination of SERS enhancement factors ${ }^{7}$.

Fig. 6.15 shows the resonance effect on the Raman activity and the depolarisation ratio, which shines more light on the coupling of the Raman modes to the resonances. Focusing on the Raman activity (top plot), it is clear that most modes increase their cross-sections monotonically when approaching the resonance edge. The $409 \mathrm{~cm}^{-1}$ and $632 \mathrm{~cm}^{-1}$ modes, however, drop in Raman activity just prior to the first resonance. These modes have, in the static limit, a negligible tensor component along the transition dipole of the first resonance at $260 \mathrm{~nm}$, and hence do not profit from the early resonance. The remaining modes all have a small tensor contribution which is compatible with the transition dipole. Beyond the first resonance the Raman activities decrease, and subsequently boost again at the strong $240 \mathrm{~nm}$ resonance. The modifications in the tensors are also evident in the depolarisation ratios (bottom plot). As a reminder: the depolarisation ratio of $\rho=0$ corresponds to a fully isotropic tensor, $\rho=1 / 3$ is a uniaxial tensor and $\rho=3 / 4$ is a zero-trace tensor. Note, for example, how the $1626 \mathrm{~cm}^{-1}$

\footnotetext{
${ }^{7}$ For example, one can not simply deduce a meaningful enhancement through the comparison of a non-resonant Raman signal to the SERS-signal in pre-resonance conditions.
} 


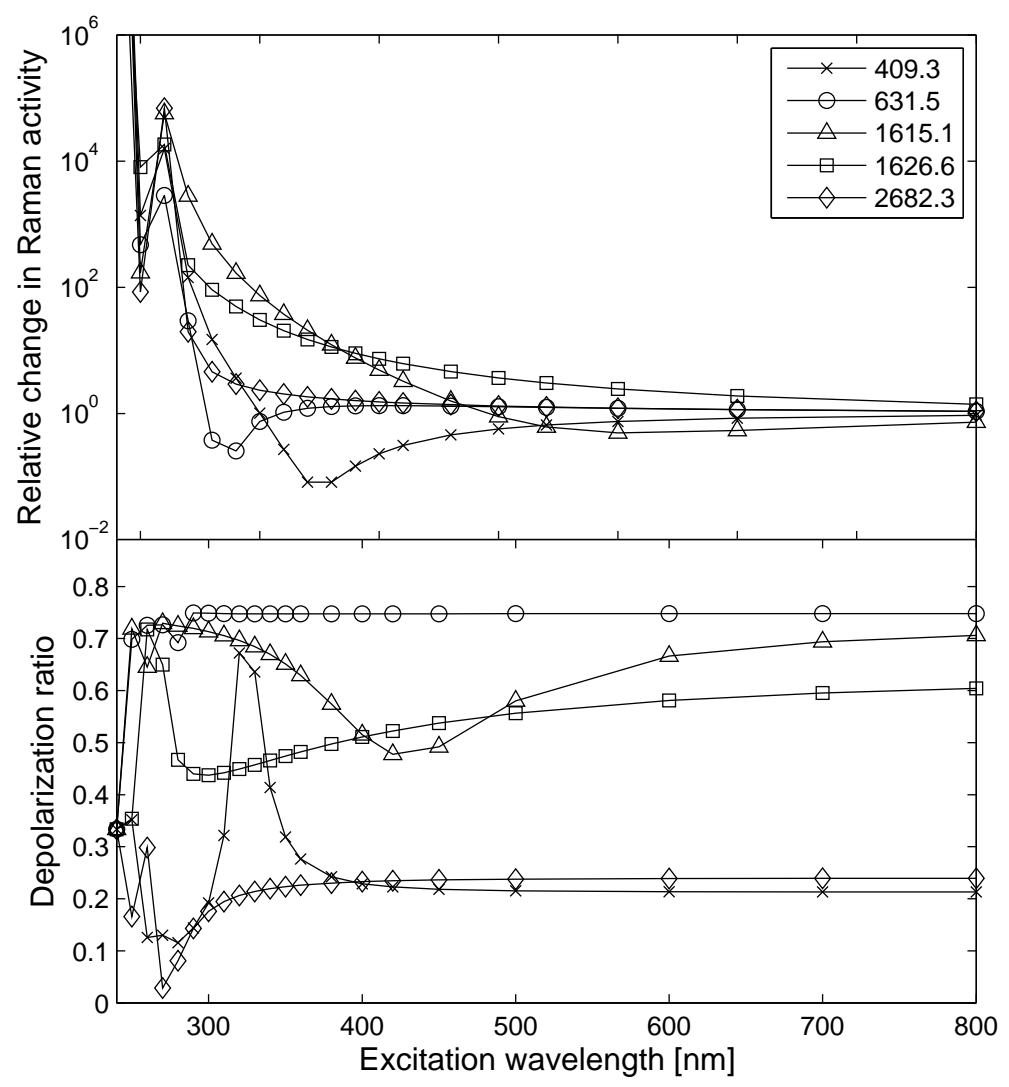

Figure 6.15 - Excitation wavelength dependence of (top) the change in Raman activity (Eq. 6.5) relative to the off-resonance situation, and (bottom) the depolarization ratio (Eq. 6.6). Both plots show the pre-resonance and resonance behavior of five selected normal modes of benzenethiol, as obtained by CPHF calculations. Note the logarithmic scale on the y-axis of the top plot - the Raman activity boosts by a factor of up to $\sim 10^{13}$ (not shown) at an excitation wavelength of $240 \mathrm{~nm}$. Also note that all modes at this excitation wavelength show a depolarization ratio of exactly $1 / 3$. 


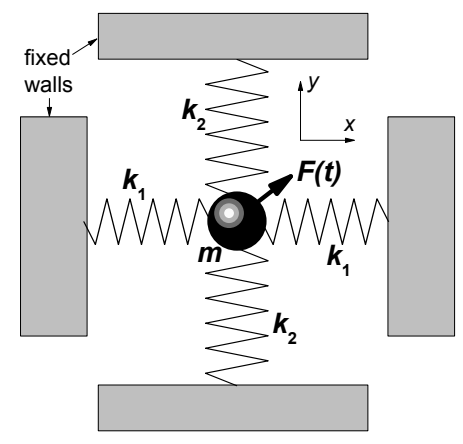

Figure 6.16 - Toy-model of the resonance conditions of a molecule. The mass $m$ is driven by an oscillation force $\vec{F}$, which results in an oscillation of the mass along $\overrightarrow{A_{m}}$. If $\vec{F}$ oscillates with $\omega_{1}=\sqrt{2 k_{1} / m}$, then $\overrightarrow{A_{m}} \| \overrightarrow{e_{x}}$, irrespective of the direction of $\vec{F}$, as long as $\vec{F} \cdot \overrightarrow{e_{x}} \neq 0$. Similarly, for $\omega_{2}=\sqrt{2 k_{2} / m}, m$ will oscillate along $y$.

mode couples early to the strong resonance at $240 \mathrm{~nm}$, before experiencing the influence of the weaker resonance in the proximity of $260 \mathrm{~nm}$. At the strong resonance however, all modes are, as evident from the tensors shown earlier, uniaxial with exactly $\rho=1 / 3$.

Apart from the tensorial re-shaping into uniaxiality, there is a simple, mechanical toy-model which, phenomenologically, explains the re-alignment of the vibration with the transition dipoles, as well as the resonant enhancement: Fig. 6.16 depicts a mass $m$ connected to fixed walls. This system has two resonance frequencies, $\omega_{1}=\sqrt{2 k_{1} / m}$ and $\omega_{2}=\sqrt{2 k_{2} / m}$, and the geometrical arrangement of springs assigns these resonances to two different directions. It is intuitive that for a driving force $\vec{F}=\vec{F}_{0} \exp (-i \omega t)$, with $\omega^{\prime} \mathrm{s}$ far from resonance, the mass will oscillate along $\vec{F}_{0}$. Pre-resonance, the amplitude of the $m^{\prime}$ s oscillation, $\overrightarrow{A_{m}}$, will increase the more $\overrightarrow{F_{0}}$ is aligned with the x- or y-axis. Moreover, $\overrightarrow{A_{m}}$ will no longer be parallel to $\overrightarrow{F_{0}}$. At $\omega_{1}$, $\overrightarrow{A_{m}}$ will be aligned with the x-axis, irrespective of the direction of $\overrightarrow{F_{0}}$, as long as $\vec{F}_{0}$ has a minimal coupling to the resonance, i.e. a non-zero component on the x-axis. Conversely, the resultant oscillation $\overrightarrow{A_{m}}$ will align with the y-axis, if $\vec{F}$ oscillates with $\omega_{2}$.

This analogy, translated back to the molecular case, means: any molecular 
vibration which has at least a minimal amount of coupling to a resonance, will be completely dominated by its symmetry. Raman polarisabilities hence align with transition dipoles, rendering the Raman tensor uniaxial, with the principal axis parallel to the transition dipole.

\subsection{Conclusion}

DFT is a useful tool in Raman spectroscopy: (i) as a teaching device, (ii) in the prediction of spectra, (iii) in the identification of closely related moieties (e.g. isotopic substitution) and (iv) in the validation of the crosssection of reference compounds. It was shown that the calculations yield detailed information on normal modes and Raman tensors, which are not directly accessible through experiment. The detailed knowledge can, in some cases, provide possible explanations for experimental observations, for example: the binding of benzenethiol to a surface which is supported by the normal mode of its vibration at $\sim 930 \mathrm{~cm}^{-1}$.

The visualisation of Raman tensors assuming SERS conditions helps identifying correlations in relative peak intensity fluctuations, which -in principleprovides a handle for the elucidation of the molecular orientation. The fact that SM-SERS requires resonance conditions, however, relativises the applicability of this approach. In the previous section, it was shown that $R a-$ man tensors lose their property of being directional beacons under resonance conditions. Thus, unless one is able to observe SM-SERS under off-resonance conditions, the correlational analysis of peak intensity fluctuations is not expected to yield much contrast; modes are expected to correlate in their fluctuations under resonance. The experimental confirmation of these findings is pending, as it is difficult to isolate the peak fluctuations from background fluctuations, as was mentioned above. 


\section{Chapter 7}

\section{Conclusions}

In this thesis the findings are summarised at the end of chapters 3-6. However, for the sake of convenience and as a service to the reader, I will repeat and summarise the most important conclusions here.

Following the general introductory remarks in chapters one and two, chapter three lays the foundation for the understanding of the surface enhancement as an electromagnetic effect. The main conclusions here are:

- The enhancement observed in SERS is a largely electromagnetic effect.

- So-called electromagnetic hotspots occur on the surface, giving rise to a strong localisation of the field enhancement on the metal surface.

- Particularly strong hotspots form in the gap between a dimer of silver colloids (nanoscopic noble metal spheres).

- The polarisation of the local field cannot simply be chosen by the experimenter: it depends on the geometry of the colloidal dimer, incident wavelength and polarisation, and the orientation of the dimer with respect to the incident beam.

- The enhancement outside the hotspot is of insignificant magnitude. 
- Most of the signal (90\%) comes from the small hotspot region $(1 \%$ surface area) - therefore only a small fraction of the overall adsorbate population contributes to the SERS signal.

- The probability to observe a particular field enhancement on a point on the surface follows a long-tail distribution, which can be modelled by a truncated Pareto distribution.

These insights are of general importance for the chapters that follow. In chapter 4, the experimental investigation of Lee \& Meisel colloids as a SERS system is carried out. The conclusions are:

- Lee \& Meisel silver colloids are roughly spherical, single particles of $\sim 50 \mathrm{~nm}$ diameter.

- The effect of salt addition is to reduce the repulsive coulombic barrier between colloids, causing aggregation. This is inferred from DLVO theory and can be examined in practise using scanning electron microscopy.

- In-situ measurements of the Raman signal upon salt-addition reveal a signal enhancement which can be described by colloidal aggregation, thus linking the SERS enhancement to colloid aggregates, consistent with the previously described electromagnetic enhancement mechanism.

- The long-term stability of aggregated SERS samples can be understood through coulomb-blocking. This is a many-body effect which causes the the aggregation to self-limit in term of cluster size; it leads to SERS-active liquid samples with a long shelf-lifetime.

Chapter 5 deals with the issue of single-molecule detection in SERS. After recognising the previous approaches to this problem as unsuitable, the alternative Bi-Analyte SERS approach is explained and introduced through examplary experiments. The conclusions drawn from here can be summarised as follows: 
- The ultra-low concentration approach is not fit to determine singlemolecule sensitivity in SERS samples. Together with the strong field enhancement localisation, a sparse number density of adsorbate molecules leads to an extremely small probability to observe a signal - SERS events become as rare as experimental outliers.

- The Bi-Analyte approach relies on relatively high adsorbate concentrations and many SERS events; it identifies single-molecule SERS event with statistical soundness.

- Single-molecule SERS is readily achieved in standard SERS systems (Lee \& Meisel silver colloids).

- Single-molecule enhancement factors as low as $\sim 10^{8}$ where determined experimentally in dry and liquid systems. This is well within the theoretically predicted maximum electromagnetic enhancement of $\sim 10^{1} 0$

Chapter 6 focusses on the application of Density-functional theory (DFT) to predict and understand Raman and SERS spectra. In short, the conclusions are:

- DFT has a large didactic value and helps to understand the physics underlying molecular vibrational spectroscopies.

- For simple organic molecules, off-resonance Raman spectra are readily predicted in peak positions and Raman tensors (with the associated magnitudes of scattering cross section and depolarisation ratio).

- Under SERS conditions the Raman tensors can be visualised as a 2D surface. It thus becomes possible to visually inspect vibrational modes for their symmetry properties and, in particular, the crosscorrelation of relative peak intensities.

- Combining the predicted Raman tensors with single-molecule SERS measurements, one could in principle infer the orientation of molecules 
on the surface, this however becomes unfeasible because (i) it is hard to predict the Raman tensors of the surface complex and (ii) under resonance conditions the orientational information in the tensors is lost.

Overall, this thesis has provided new key insights into the technique of SERS (for example, the development of a more reliable method to identify single molecules). These new findings are expected to play a significant role in the further development of the technique and perhaps yet unforseen applications. 


\section{Appendix A}

\section{Molecular footprints}

[...] if all the people who go to sleep in church were laid end to end they would be a lot more comfortable.

Mrs. Robert A. Taft

We can estimate the maximum area a molecule will occupy on a surface by tightly enclosing its projection in a rectangle. Obviously, the resulting area depends strongly on a sensible choice of projection and enclosure - we chose molecular orientations which reflect worst-case scenarios, i.e. parallel rather than normal orientation with respect to the surface. Note that this may not be the physically most likely orientation.

We also arbitrarily chose an atomic 'radius' of $0.5 \AA$, which is included in the resulting projections.

The projected molecular structures are obtained from DFT calculations (unless noted otherwise, obtained at the same level of theory as used in chapter 6, namely: method B3LYP and basis set 6-311++G(d,p)).

The results for BTZ2, Rh6G $\mathrm{G}^{1}$ and CV are summarised in Figs. A.1-A.3.

\footnotetext{
${ }^{1}$ Calculation data taken from Ref [169]. The original data was kindly provided by Hiroyuki Watanabe.
} 


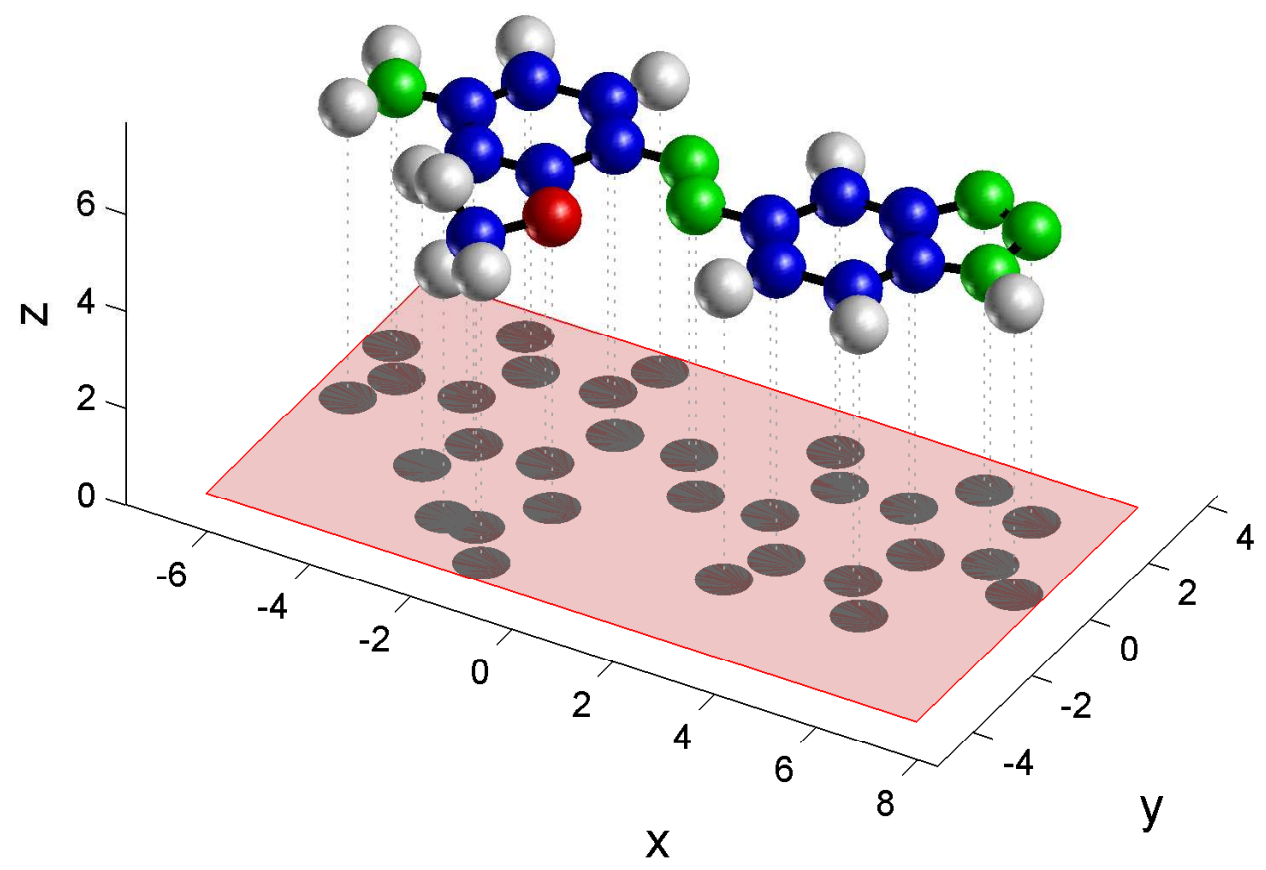

Figure A.1 - BTZ2 projected into its plane (the molecule has only two out-ofplane atoms). All length scales given in $\AA$. The plane of the molecule is offset by $6 \AA$ A with respect to the $x-y$ plane. The highlighted rectangle has an area of $1 \mathrm{~nm}^{2}$. 


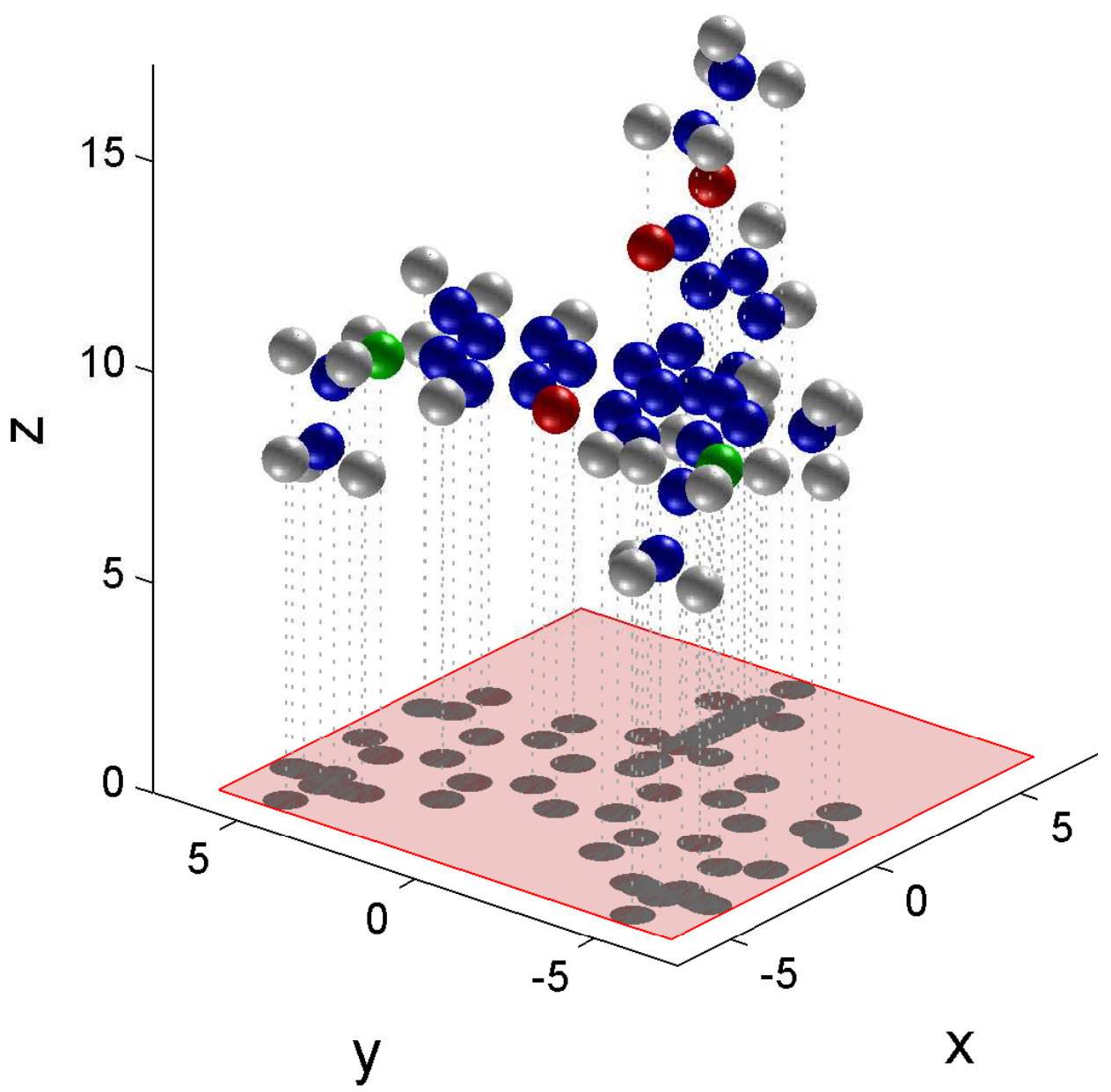

Figure A.2 - Projection of Rh6G with its backbone oriented parallel to the $x-y$ plane. Note that the ligand which is not part of the backbone is oriented perpendicular to the $x-y$ plane. All length scales given in $\AA$. The plane of the backbone is offset by $10 \AA$ with respect to the $x$-y plane. The highlighted rectangle has an area of $1.6 \mathrm{~nm}^{2}$. 


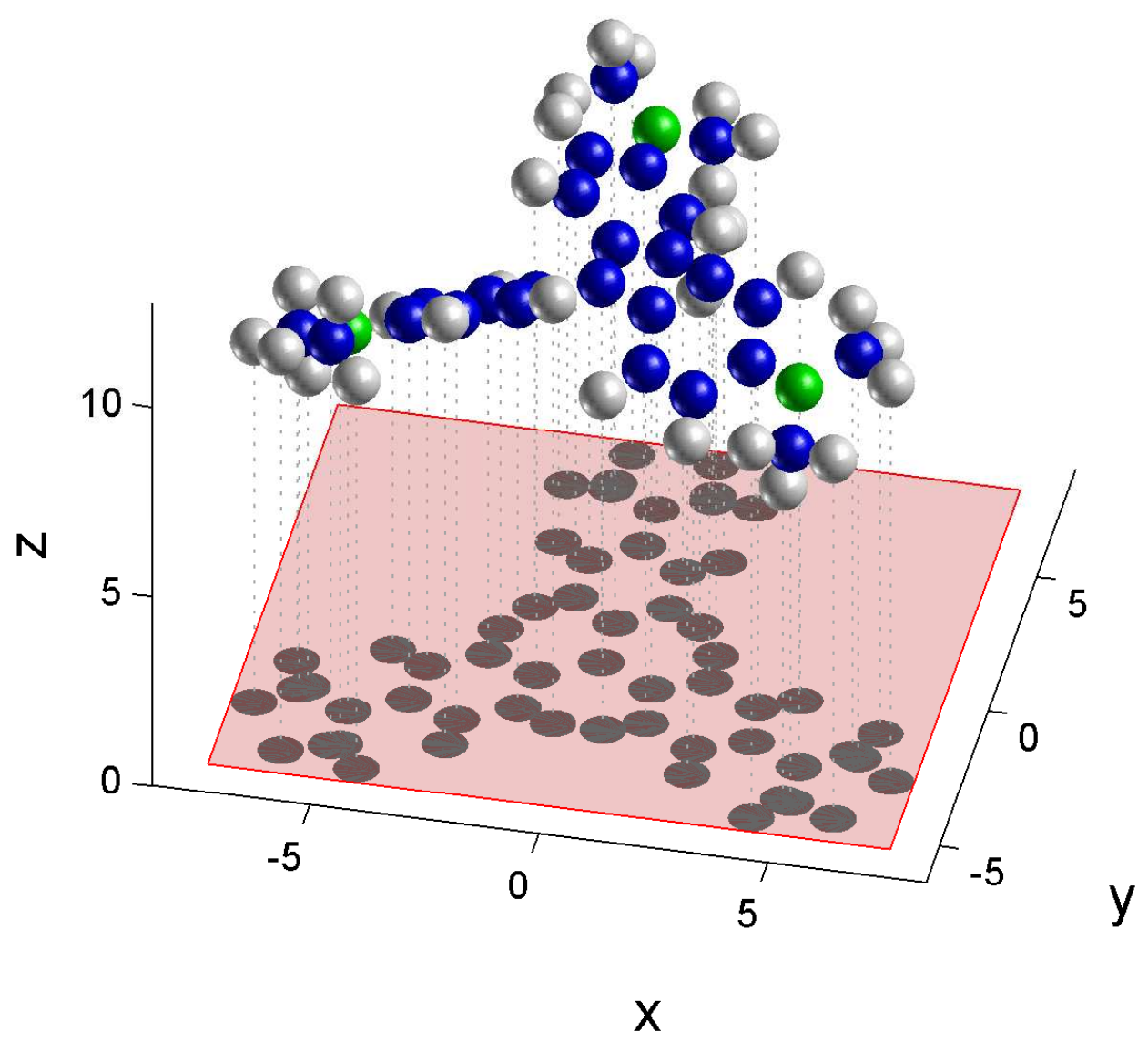

Figure A.3 - Projection of CV with its plane oriented parallel to the $\mathrm{x}$-y plane. The structure shown here was computed at a B3LYP/6-31+G(d) level. All length scales given in $\AA$. The plane of the molecule is offset by $10 \AA$ with respect to the $\mathrm{x}-\mathrm{y}$ plane. The highlighted rectangle has an area of $2 \mathrm{~nm}^{2}$. 


\section{Appendix B}

\section{BiASERS - from Pascal's triangle to Mie theory}

I find enough mystery in mathematics to satisfy my spiritual needs.

Tom Lehrer

This appendix shall give some insight into the statistics of the three models used to introduce the BiASERS technique (see Sec. 5.3.1). A detailed discussion of these statistics is not strictly necessary for the understanding of the BiASERS technique - it is, however, twined around the train of thought which led us from first hand-waving arguments to our current, more elaborate model. Also, the concepts used herein are simple and have their own mathematical charm - a property which is rarely given the attention it deserves.

\section{Closed discrete hotspots}

Sec. 5.3.2 describes the idea of a discrete ('bucket-like') hotspot. From there, simple arguments lead to a strong statistical argument which ba- 
sically proves SM-SERS. The probability to observe pure spectra if the number of molecules $N$ in the discrete hotspot is fixed (see Fig. 5.7) yields a distribution with close ties to Pascal's triangle:

\begin{tabular}{|c|c|c|c|c|c|}
\hline$N=0:$ & & & 1 & & \\
\hline$N=1:$ & & 1 & & 1 & \\
\hline$N=2:$ & 1 & & 2 & 1 & 1 \\
\hline$N=3:$ & 1 & 3 & & 3 & 1 \\
\hline$N=4: \quad 1$ & 4 & & 6 & 4 & 4 \\
\hline
\end{tabular}

where each row describes the number of permutations for a particular combination of two types of molecules. The sum of each row is $2^{N}$, the number of all possible combinations. For example, for $N=3$ (compare Fig. 5.5), this yields:

- $1 / 8$ chance to observe a pure dye A spectrum,

- 3/8 chance to observe a ' 2 dye $\mathrm{A}+1$ dye $\mathrm{B}$ ' mixed spectrum,

- 3/8 chance to observe a ' 1 dye $\mathrm{A}+2$ dye $\mathrm{B}$ ' mixed spectrum,

- $1 / 8$ chance to observe a pure dye B spectrum.

Pascal's triangle describes this model situation because the elements of the triangle are the binomial coefficients

$$
\left(\begin{array}{c}
N \\
k
\end{array}\right)=\frac{N !}{k !(N-k) !}
$$

which are interpreted as the number of k-element subsets of an N-element set. Normalised to the row-sum Pascal's triangle yields the discrete prob- 
abilities:

$$
\begin{aligned}
& N=0: \quad 1 \\
& N=1: \quad 1 / 2 \quad 1 / 2 \\
& N=2: \quad 1 / 4 \quad 1 / 2 \quad 1 / 4 \\
& N=3: \quad 1 / 8 \quad 3 / 8 \quad 3 / 8 \quad 1 / 8 \\
& N=4: 1 / 16 \quad 1 / 4 \quad 6 / 16 \quad 1 / 4 \quad 1 / 16
\end{aligned}
$$

The spectral participance of an element follows from the ratio of the element (denominator) and its upper left neighbor (nominator). For an example see the bold numbers in the triangles below: $3 / 6=1 / 2$. This strategy obviously breaks on the left edge, therefore one needs to pad the triangle's left edge with zeros:

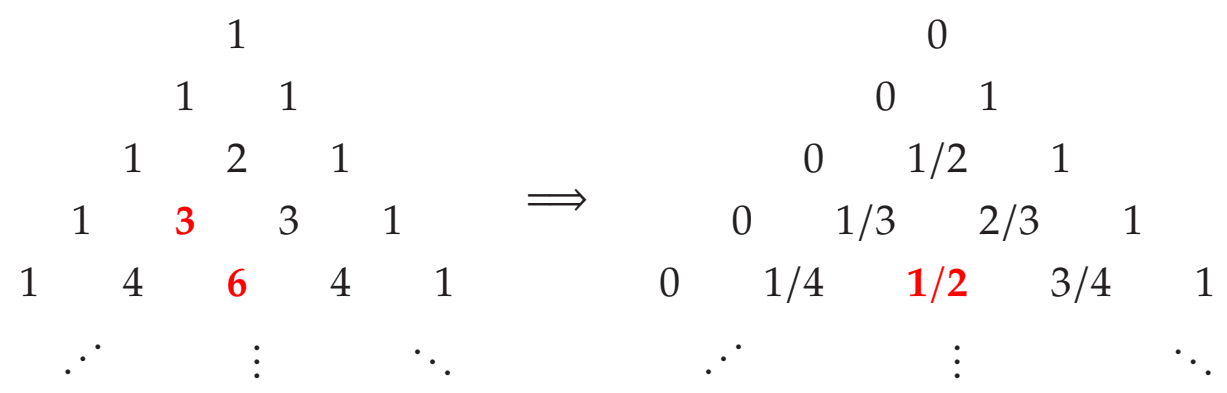

Figure B.1 shows the pattern in which this distribution follows the central limit theorem and approaches a normal distribution.

\section{Open discrete hotspots}

Obviously, due to the sample diversity in a real SERS experiment, the approximation of fixed $N$ is crude. In a first step towards more realism, one can, while retaining the fixed extent of the hotspot, allow the number of molecules per hotspot to be Poisson distributed $\left(f(k, \lambda)=\frac{\lambda^{k} e^{-\lambda}}{k !}\right)$. Now, $\lambda$ replaces the notion of $N$. Observing the principle of independently adsorbing dye species, a Poisson distribution for each of the two dyes is assumed, with potentially different parameters $\lambda_{A}$ and $\lambda_{B}$. 


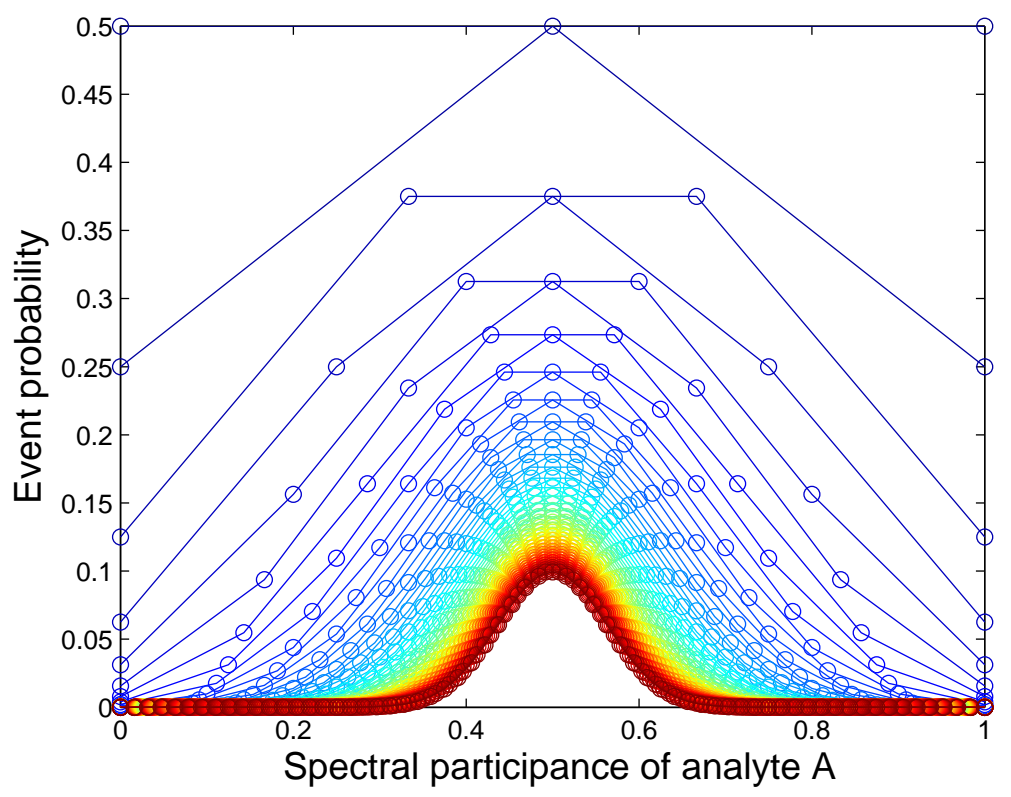

Figure B.1 - Probability distributions of the spectral participance in events, assuming a closed, discrete hotspot (fixed $N$ ), for all $N$ from 1 to 64 .

The probability to observe, for example, a spectrum from 2 dyes A and 1 dye B now becomes:

$$
p\left(2, \lambda_{A}, 1, \lambda_{B}\right)=\frac{\lambda_{A}^{2} e^{-\lambda_{A}}}{2 !}+\frac{\lambda_{B}^{1} e^{-\lambda_{A}}}{1 !}
$$

The binomial distribution is now replaced by Poissonian probability (for the sake of a terse notation, the $\lambda^{\prime}$ s are omitted, so that $p\left(a, \lambda_{A}, b, \lambda_{B}\right) \Rightarrow$ $p(a, b)$.):

$$
\begin{aligned}
& p(0,0) \\
& p(0,1) \quad p(1,0) \\
& p(0,2) \quad p(1,1) \quad p(2,0) \\
& p(0,3) \quad p(1,2) \quad p(2,1) \quad p(3,0) \\
& p(0,4) \quad p(1,3) \quad p(2,2) \quad p(3,1) \quad p(4,0)
\end{aligned}
$$

To obtain the event probability distribution, it is now necessary to histogram, according to spectral percentage, the summed probability from all elements in the triangle. A few example combinations of $\lambda_{A}$ and $\lambda_{B}$, 


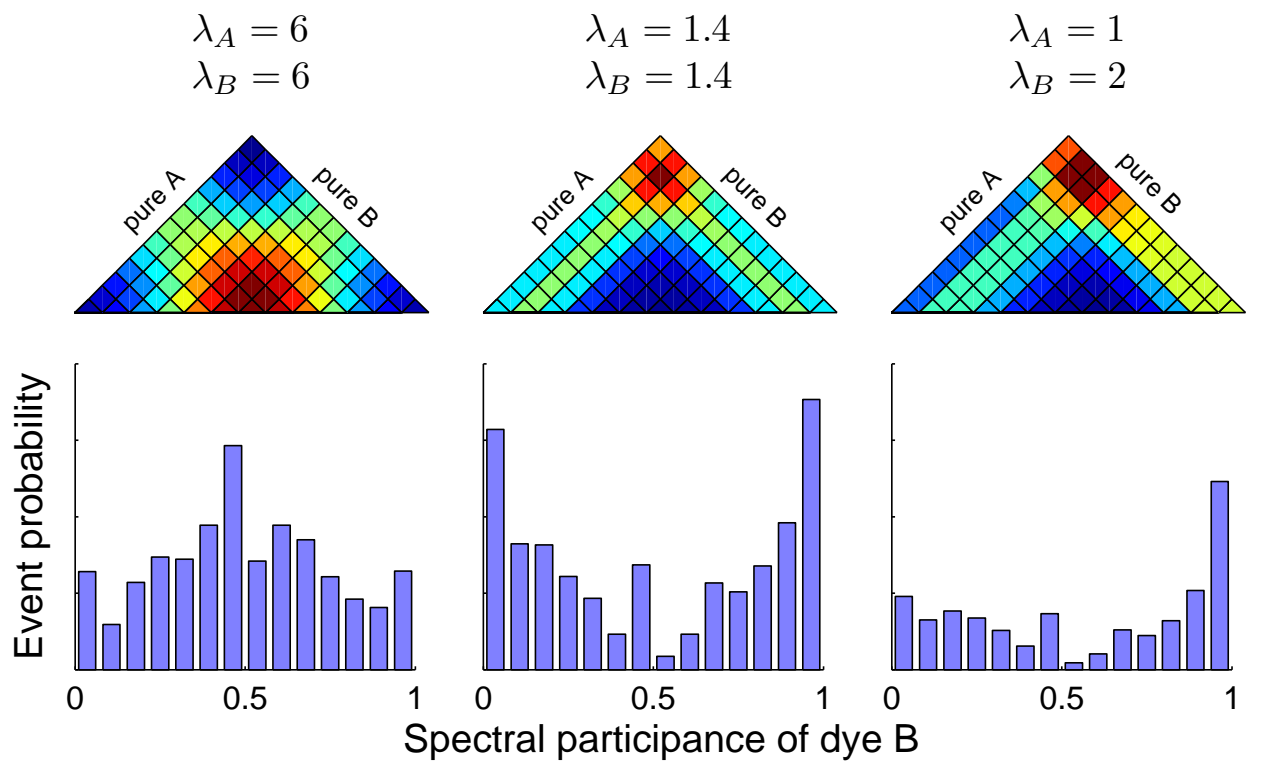

Figure B.2 - Top: Probability distributions for molecular combinations based on Poisson statistics for each analyte. See text for details. Bottom: resulting histogram reflecting the probability to observe events as a function of the spectral participance. The apparent asymmetry in the histograms is due to binning ( - it is non-trivial to find evenly-space bin-boundaries upon which no data points come to lie.)

together with their resultant distribution and histograms, are given in Fig. B.2.

\section{Open continuous hotspots}

Treating hotspots as buckets is, without doubt, a crude simplification. For the sake of a better model the notion of a hotspot with fixed extent must be dropped, and replaced with a position dependent enhancement factor $f(\vec{r})$. For the typical example of a spherical colloid, one can define $f(\vartheta, \varphi)$, where $\vartheta$ and $\varphi$ are spherical coordinates and the hotspot center comes to lie at $\vartheta=0$.

With the sharp hotspot boundaries gone, one must consequently drop 
the discrete Poisson distribution of molecules - how can one decide how many molecules are within a hotspot, if the hotspot has no limits? The only permissible notion of a discrete probability distribution now lies in the fact that the number of molecules per colloid is discrete, even limited, but of little interest in a situation where most of the signal originates from a small fraction of the overall surface area.

The simplest, and arguably most practical approach is to solve the problem numerically. Assume the expected enhancement $f(\vartheta, \varphi)$ to be derived from Generalised Mie Theory, and the (average) numbers $\mathbb{N}_{A}$ and $\mathbb{N}_{B}$ of molecules per colloid to be fixed, for analyte A and B. To simulate one event, one can now:

1. give each molecule random coordinates $\vartheta_{i}$ and $\varphi_{i}$, evenly distributed on the surface of the sphere,

2. evaluate the signal to be obtained from each molecule: $f\left(\vartheta_{i}, \varphi_{i}\right)$,

3. sum the contributions into overall signals per analyte, $F_{A}$ and $F_{B}$,

4. the observed spectral participation is then: $P_{A}=\frac{F_{A}}{\left(F_{A}+F_{B}\right)}$

To evaluate event probabilities, this run needs to be repeated many times - the more, the better the statistics. In this sense, this strategy become as Monte-Carlo simulation. For an exemplary application, see Ref. [113], in particular the supporting information of this paper. 


\section{Appendix C}

\section{Artwork}

There is no science without fancy and no art without fact.

Vladimir Nabokov

One of the most important aspects of scientific work lies in its communication to others: to the scientific community in a specialised field, or scientists in general, or even the general public. In this case, the target audience is the specialised community. Being part of it, it is easy to cast a wall of graphs, equations and diagrams, i.e. a peer-reviewed paper, at the audience. In turn, it comes as a challenge if, after a publication is accepted, the journal asks for a cover image - which shall achieve the split between being an eye-catcher to every scientist in the field, and yet having a clear connection to the usually fairly distinct topic at hand. This challenge comes with a great reward ${ }^{1}$ : the freedom of dropping accuracy in favour of beauty, casting scientific ideas into pictures without worrying about the details, gives the mind a toy-model of the complex, a visual vocabulary which feeds intuition.

Apart from the drafting pen and paper, these images were created using

\footnotetext{
${ }^{1}$ It also comes at a cost: from experience, explaining the fact that the author provides the journal with design work, and pays the journal for it, only elucidates disbelief and nervous laughter from the uninitiated.
} 
free, open source software [170,171]. The following pages document the artwork created by the author during the course of the work undertaken for this thesis. 


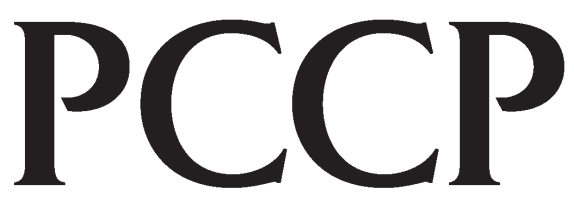

Physical Chemistry Chemical Physics

An international journal

www.rsc.org/pecp

Volume 9 | Number 23 | 21 June 2007 | Pages 2905-3044

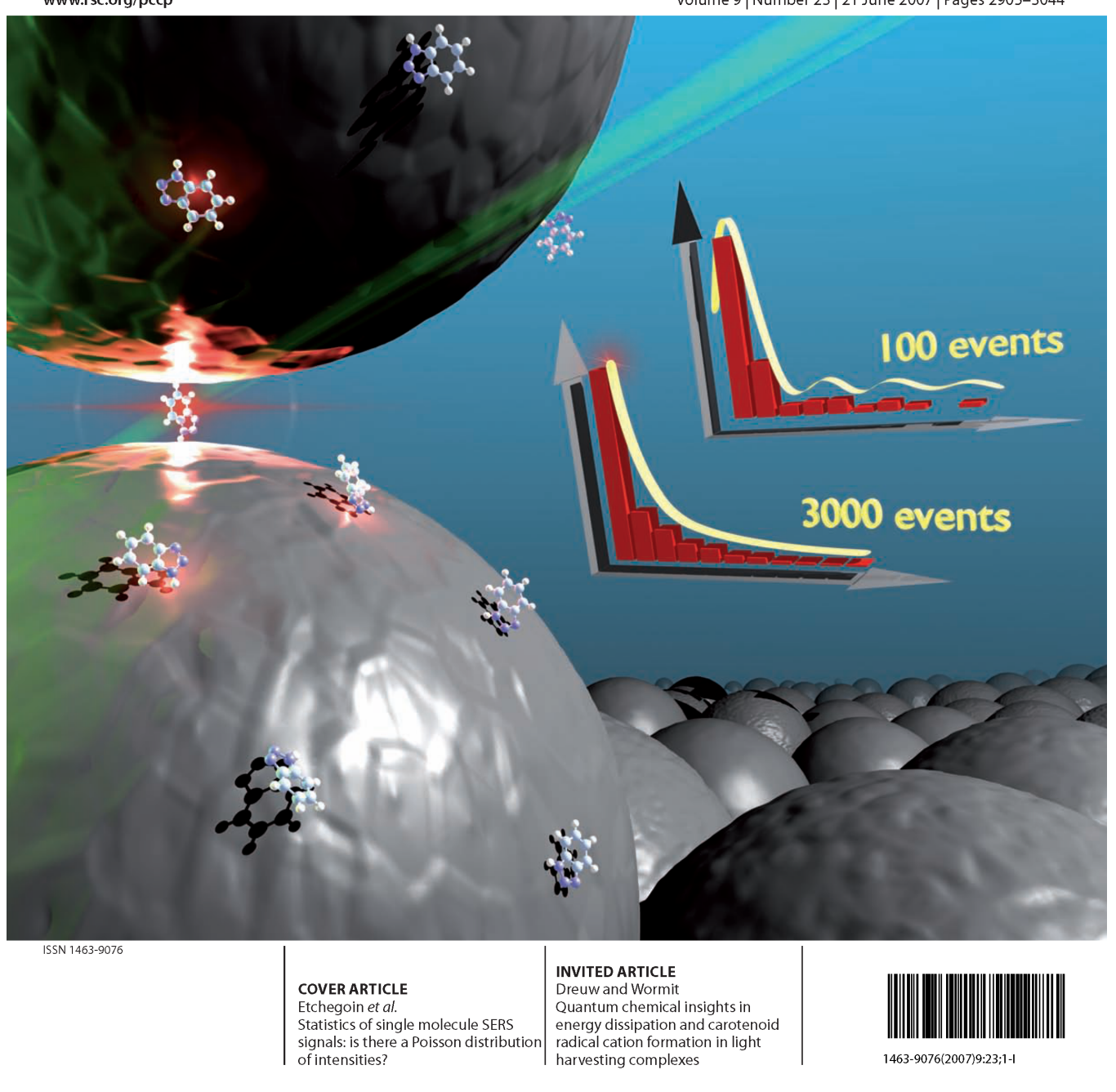

Figure C.1 - Cover of Phys. Chem. Chem. Phys. 9 (23), June 2007. See Ref. [75]. 


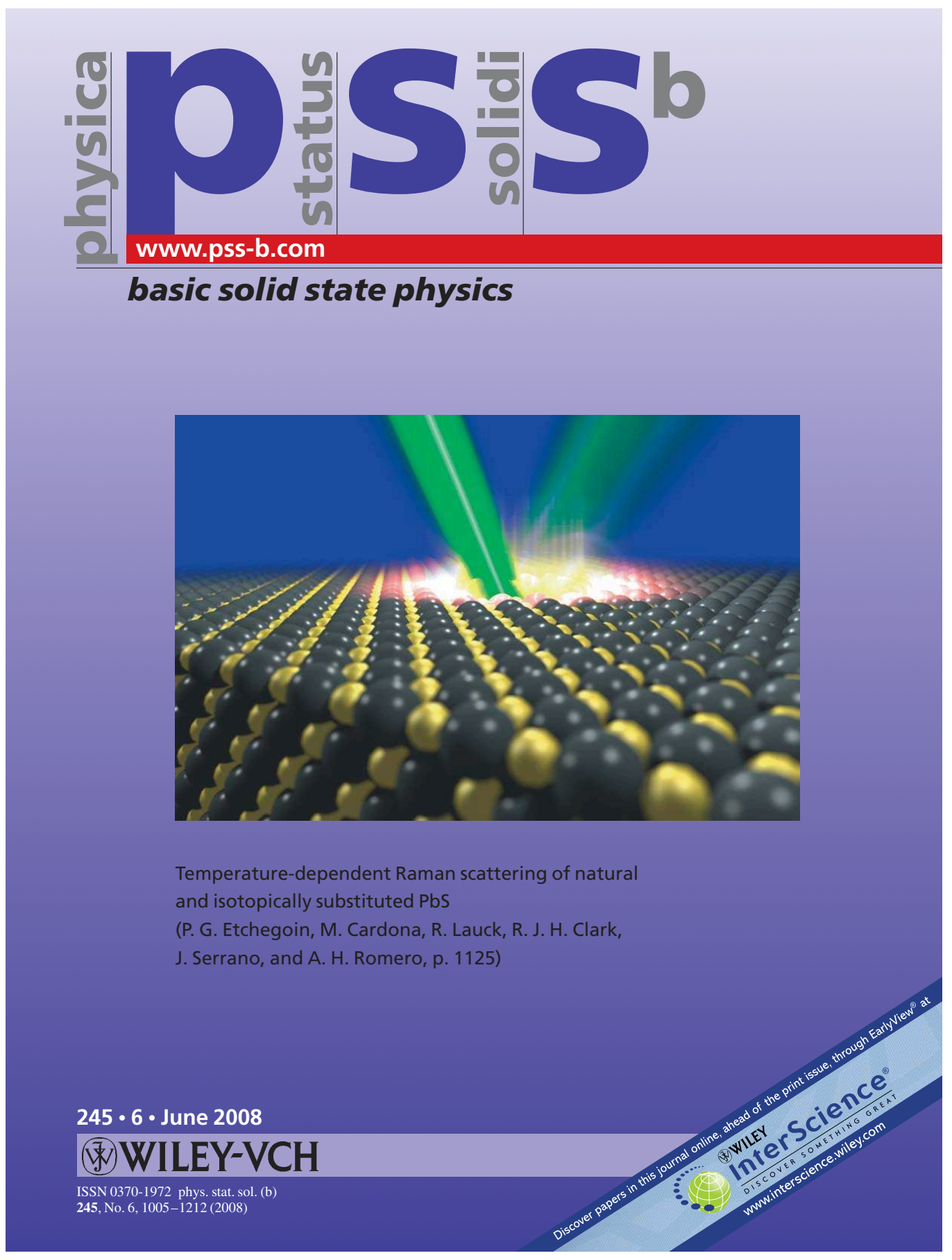

Figure C.2 - Cover of Phys. Chem. Chem. Phys. 10 (28), July 2008. See Ref. [172]. 


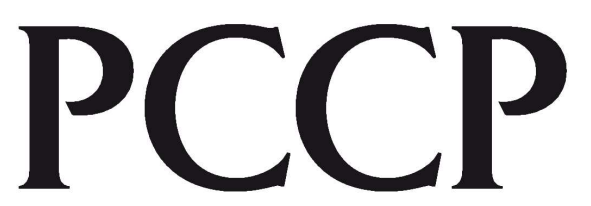

Physical Chemistry Chemical Physics

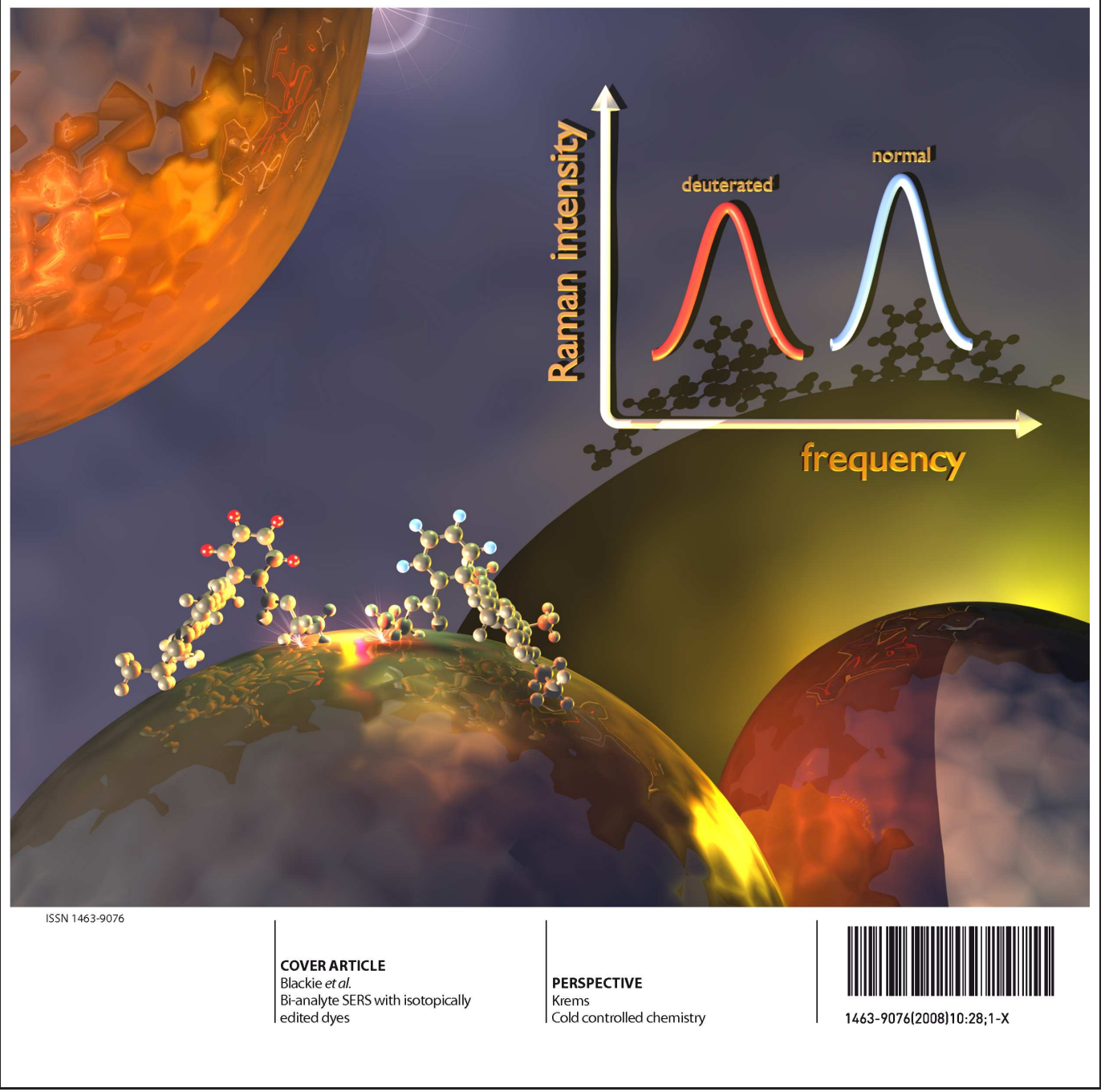

Figure C.3 - Cover of Phys. Chem. Chem. Phys. 10 (28), July 2008. See Ref. [148]. 


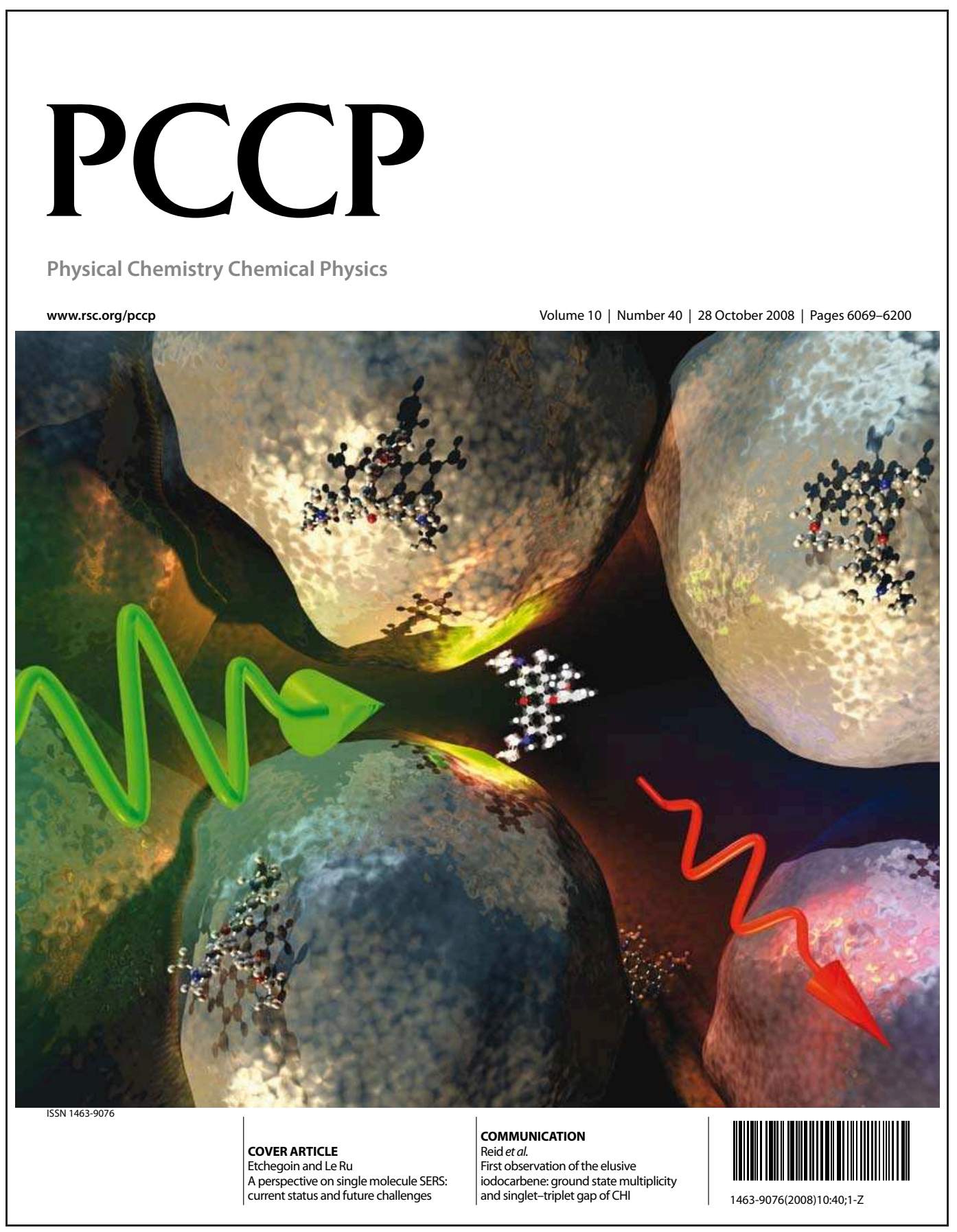

Figure C.4 - Cover of Phys. Chem. Chem. Phys. 10 (40), October 2008. See Ref. [173]. 


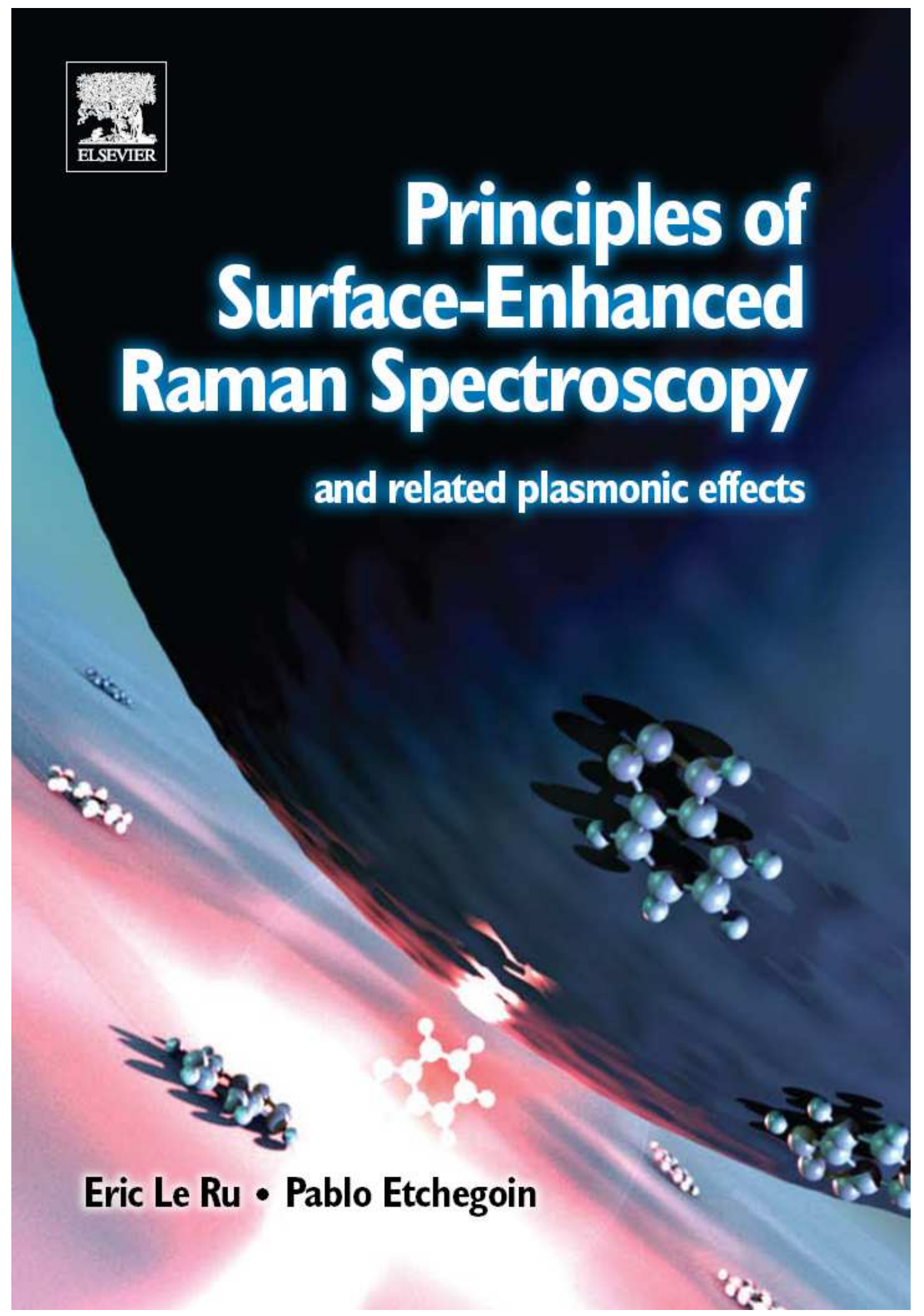

Figure C.5 - Cover of Eric Le Ru's and Pablo Etchegoin's book, see Ref. [17]. 208 


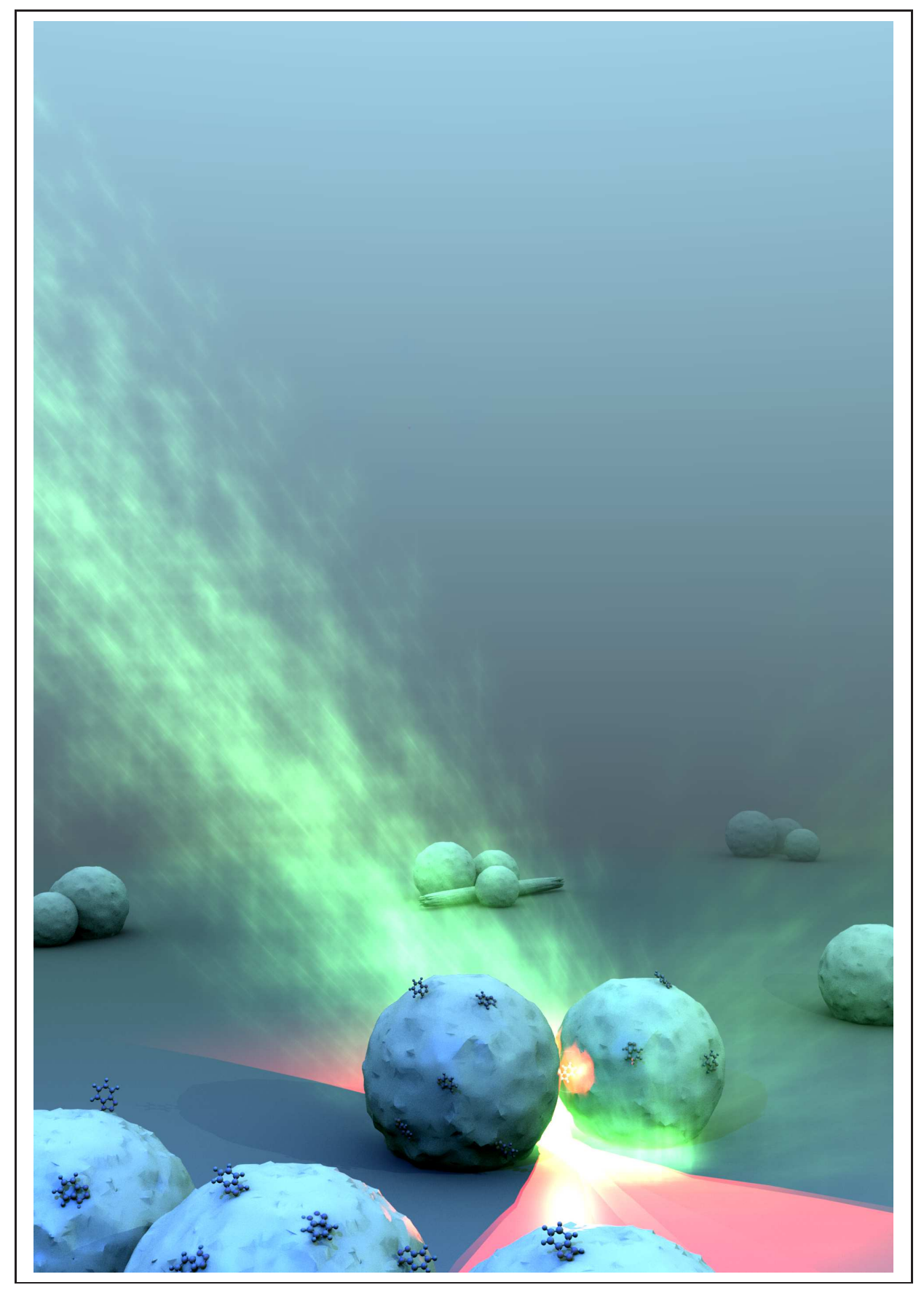

Figure C.6 - Unpublished cover concept. 

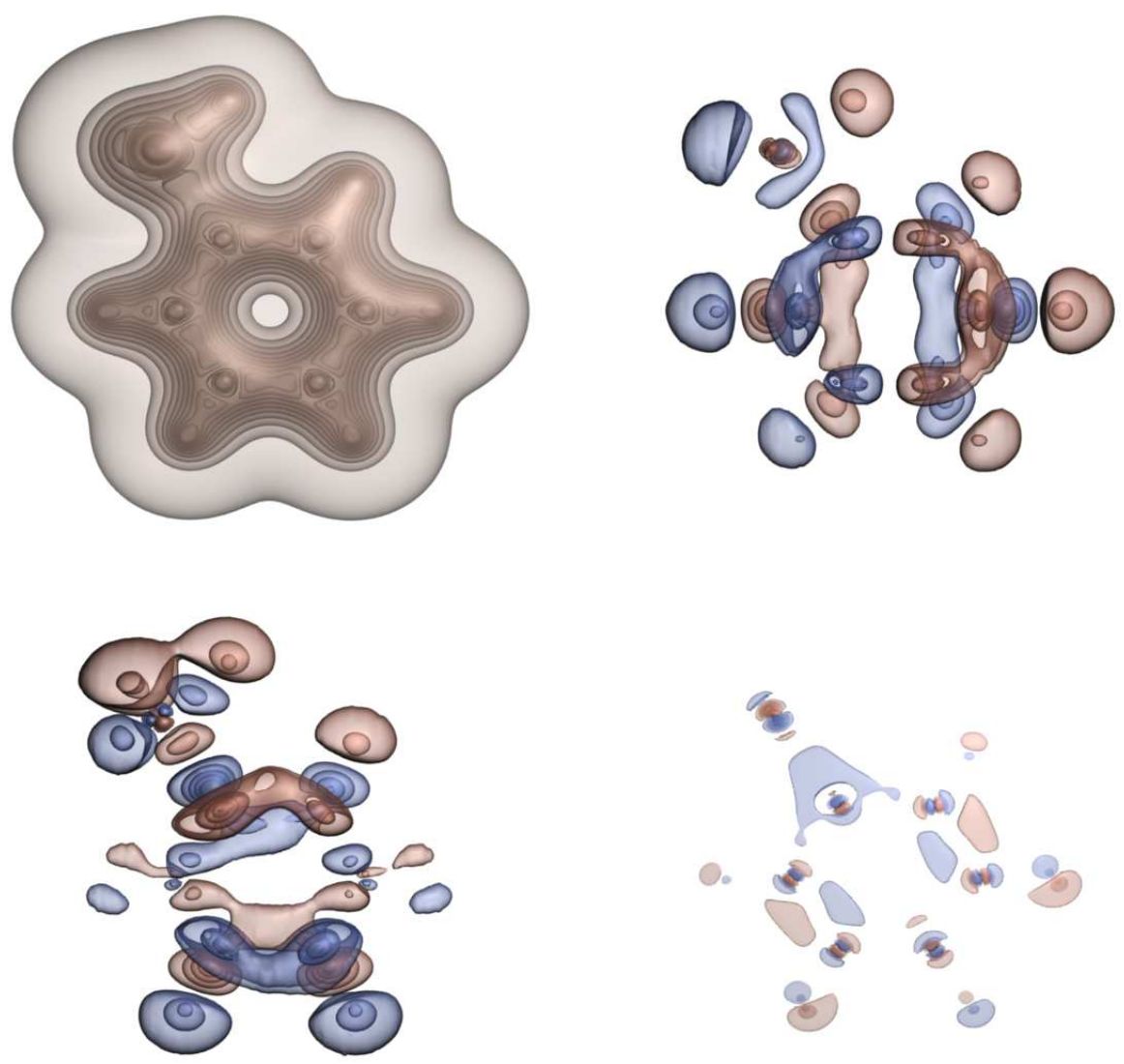

Figure C.7 - Ray-traced rendering [170] of semi-transparent isosurfaces of the electron density and difference electron densities of benzenethiol under various perturbations. 


\section{Appendix D}

\section{Publications}

What follows are the headers of the papers published during the course of this $\mathrm{PhD}^{1}$, in chronological order:

Self-Limiting Aggregation Leads to Long-Lived Metastable Clusters in Colloidal Solutions

M. Meyer,* E. C. Le Ru, ${ }^{\dagger}$ and P. G. Etchegoin*

The MacDiarmid Institute for Advanced Materials and Nanotechnology, School of Chemical and Physical Sciences, Victoria University of Wellington, P.O. Box 600 Wellington, New Zealand

Received: October 13, 2005; In Final Form: January 24, 2006

The existence of a metastable state with limited Coulomb-blocked aggregation at the onset of instability in a colloidal solution is proposed and demonstrated both experimentally and theoretically (through Monte Carlo simulations). Such a stable state of small clusters of metallic colloids happens to be extremely important for techniques such as surface-enhanced Raman scattering (SERS), which profits explicitly from collective plasmon resonances in these clusters to boost Raman signals of specific analytes. In fact, SERS provides a unique tool to understand, monitor, and study the onset of aggregation in colloidal silver/gold and to prove the existence of the proposed state at the boundary of colloid coalescence.

\footnotetext{
${ }^{1}$ Due to the format of the paper, a header for Ref. [80] is absent from this list.
} 
1944

J. Phys. Chem. B 2006, 110, 1944-1948

Proof of Single-Molecule Sensitivity in Surface Enhanced Raman Scattering (SERS) by Means of a Two-Analyte Technique

E. C. Le Ru,* M. Meyer, and P. G. Etchegoin*

The MacDiarmid Institute for Advanced Materials and Nanotechnology, School of Chemical and Physical Sciences, Victoria University of Wellington, P.O. Box 600, Wellington, New Zealand

Received: August 22, 2005; In Final Form: October 27, 2005

A method is proposed to pin down unambiguous proof for single-molecule sensitivity in surface enhanced Raman spectroscopy (SERS). The simultaneous use of two analyte molecules enables a clear confirmation of the single (or few)-molecule nature of the signals. This method eliminates most of the uncertainties associated with low dye concentrations in previous experiments. It further shows that single- or few-molecule signals are very common in SERS, both in liquids and on dry substrates.

THE JOURNAL OF CHEMICAL PHYSICS 125, 204701 (2006)

\section{Enhancement factor distribution around a single surface-enhanced Raman scattering hot spot and its relation to single molecule detection}

E. C. Le Ru, ${ }^{\text {a) }}$ P. G. Etchegoin, ${ }^{\text {b) }}$ and M. Meyer

The MacDiarmid Institute for Advanced Materials and Nanotechnology, School of Chemical and Physical

Sciences, Victoria University of Wellington, P.O. Box 600, Wellington 6140, New Zealand

(Received 11 August 2006; accepted 12 October 2006; published online 22 November 2006)

We provide the theoretical framework to understand the phenomenology and statistics of single molecule (SM) signals arising in surface enhanced Raman scattering (SERS) under the presence of so-called electromagnetic hot spots. We show that most characteristics of the SM-SERS phenomenon can be tracked down to the presence of a tail-like (power law) distribution of enhancements and we propose a specific model for it. We analyze, in the light of this, the phenomenology of SM-SERS and show how the different experimental manifestations of the effect reported in the literature can be analyzed and understood under a unified "universal" framework with a minimum set of parameters. (C) 2006 American Institute of Physics. [DOI: $10.1063 / 1.2390694]$

THE JOURNAL OF CHEMICAL PHYSICS 125, 164705 (2006)

\section{An analytic model for the optical properties of gold}

P. G. Etchegoin, ${ }^{\text {a) }}$ E. C. Le Ru, ${ }^{\text {b) }}$ and M. Meyer

The MacDiarmid Institute for Advanced Materials and Nanotechnology, School of Chemical and Physical

Sciences, Victoria University of Wellington, P.O. Box 600, Wellington, New Zealand

(Received 1 August 2006; accepted 11 September 2006; published online 24 October 2006)

[DOI: $10.1063 / 1.2360270]$ 
Surface Enhanced Raman Scattering Enhancement Factors: A Comprehensive Study

E. C. Le Ru,* E. Blackie, M. Meyer, and P. G. Etchegoin ${ }^{\dagger}$

The MacDiarmid Institute for Advanced Materials and Nanotechnology, School of Chemical and Physical Sciences, Victoria University of Wellington, PO Box 600, Wellington 6140, New Zealand

Received: December 21, 2006; In Final Form: July 17, 2007

This paper presents an in-depth study of Surface Enhanced Raman Scattering (SERS) enhancement factors (EFs) and cross-sections, including several issues often overlooked. In particular, various possible rigorous definitions of the SERS EFs are introduced and discussed in the context of SERS applications, such as analytical chemistry and single molecule SERS. These definitions highlight the importance of a careful characterization of the non-SERS cross-sections of the probes under consideration. This aspect is illustrated by experimental results for the non-SERS cross-sections of representative SERS probes along with average SERS EFs for the same probes. In addition, the accurate experimental determination of single molecule enhancement factors is tackled with two recently developed techniques, namely: bi-analyte SERS (BiASERS) and temperaturedependent SERS vibrational pumping. We demonstrate that SERS EFs as low as $10^{7}$, as opposed to the figure of $10^{14}$ often claimed in the literature, are sufficient for the observation of single molecule SERS signals, with maximum single molecule EFs typically on the order of $\sim 10^{10}$.

\section{Statistics of single molecule SERS signals: is there a Poisson distribution of intensities?}

\section{P. G. Etchegoin,* M. Meyer and E. C. Le Ru*}

Received 16th March 2007, Accepted 11th April 2007

First published as an Advance Article on the web 4th May 2007

DOI: $10.1039 / \mathbf{b} 704013 \mathrm{j}$

This paper is aimed at clarifying the statistics of single molecule (SM) surface enhanced Raman scattering (SERS) signals. The argument of the possible existence of a Poisson distribution in the statistics of intensities in SM-SERS has been used many times in the last decade as a proof of single molecule detection. We show theoretically and experimentally that the conditions under which a Poisson distribution would be present are so unlikely to exist in a real system that there is no other option but to attribute the claims to poor statistical sampling. We believe the argument based on Poisson statistics should be dropped as a proof of single molecule detection in SERS. 
Anal. Chem. 2007, 79, 8411-8415

Technical Notes

\title{
Statistics of Single-Molecule Surface Enhanced Raman Scattering Signals: Fluctuation Analysis with Multiple Analyte Techniques
}

\author{
P. G. Etchegoin,* M. Meyer, E. Blackie, and E. C. Le Ru \\ The MacDiarmid Institute for Advanced Materials and Nanotechnology, School of Chemical and Physical Sciences,
} Victoria University of Wellington, P.O. Box 600 Wellington, New Zealand

The mathematical background, based on a variation of the principal component analysis (PCA) method, is developed for the understanding of fluctuating multiple analyte single-molecule (SM) surface enhanced Raman scattering (SERS) signals; with emphasis on the bianalyte SERS technique developed recently. The method and its significance are presented to provide a systematic framework with which several aspects of the statistics of SM-SERS signals can be analyzed in general. We also apply the method to a concrete example of bianalyte statistics in silver colloidal solutions and discuss related topics around experimental issues and the interpretation of singlemolecule SERS data. that purpose by other authors, ${ }^{5,6}$ but a few questions remain; among them: (i) how much can we obtain or learn from the statistics of single-molecule events in BiASERS? and (ii) is there a systematic and reliable method for the study of single-molecule statistics in SERS from two or many analytes?

It is the purpose of this paper to develop further the ideas and tools needed to understand single-molecule fluctuations in SERS with multiple analytes. To this end, we shall develop an analysis tool based on a variation of the principal component analysis (PCA) method. We shall assume no previous exposure to the PCA method and, therefore, we shall explain the relevant steps in some detail in the Supporting Information companion material to this paper, making emphasis on the physical meaning of the results. Therein the method is tested in a "model" examnle of simulated

THE JOURNAL OF CHEMICAL PHYSICS 127, 189901 (2007)

Erratum: "An analytic model for the optical properties of gold" [J. Chem. Phys. 125, 164705 (2006)]

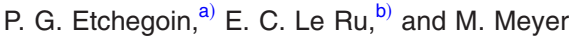

The MacDiarmid Institute for Advanced Materials and Nanotechnology, School of Chemical and Physical Sciences, Victoria University of Wellington, P.O. Box 600, Wellington, New Zealand

(Received 27 September 2007; accepted 3 October 2007; published online 9 November 2007)

[DOI: $10.1063 / 1.2802403]$ 
JOURNAL OF RAMAN SPECTROSCOPY

J. Raman Spectrosc. 2008; 39: 1127-1134

Published online 26 March 2008 in Wiley InterScience

(www.interscience.wiley.com) DOI: $10.1002 /$ jrs.1945

JRS

\title{
Advanced aspects of electromagnetic SERS enhancement factors at a hot spot
}

\section{E. C. Le Ru, ${ }^{*}$ M. Meyer, E. Blackie and P. G. Etchegoin}

The MacDiarmid Institute for Advanced Materials and Nanotechnology, School of Chemical and Physical Sciences, Victoria University of Wellington, Wellington, New Zealand

Received 18 September 2007; Accepted 2 December 2007

\begin{abstract}
In this paper, we discuss some advanced theoretical aspects of electromagnetic enhancement factors (EFs) in surface-enhanced Raman scattering (SERS). We focus in particular on the influence of surface selection rules (SSRs) on SERS EFs at hot spots, and the determination of SERS depolarization ratios. Both aspects could be viewed as secondary (compared to the overall magnitude of the SERS EF), but are nevertheless observable experimentally and crucial for a fundamental understanding of SERS. They also share the property that they cannot be studied within the commonly used $|E|^{4}$ approximation to the SERS EFs, and appropriate tools are developed here to make predictions beyond this approximation in the case of a SERS hot spot. In addition, theoretical estimates of different types of (previously defined) EFs are provided, and their origins discussed for the typical example of a SERS substrate dominated by SERS hot spots. Finally, experimental measurements of SERS depolarization ratios are presented to support the theoretical predictions. Copyright $\odot 2008$ John Wiley \& Sons, Ltd.
\end{abstract}

\section{Bi-analyte SERS with isotopically edited dyes}

E. Blackie, ${ }^{* a b}$ E. C. Le Ru ${ }^{a b}$ M. Meyer, ${ }^{a b}$ M. Timmer, ${ }^{b c}$ B. Burkett, ${ }^{b}$

P. Northcote ${ }^{b}$ and P. G. Etchegoin ${ }^{a b}$

Received 4th March 2008, Accepted 21st April 2008

First published as an Advance Article on the web 3rd June 2008

DOI: $10.1039 / \mathbf{b 8 0 3 7 3 8 h}$

Isotopically substituted rhodamine dyes provide ideal probes for the study of single-molecule surface enhanced Raman scattering (SM-SERS) events through multiple-analyte techniques.

Isotopic editing should, in principle, provide probes that have identical chemical properties (and surface chemistries); while exhibiting at the same time distinct Raman features which enable us to identify single-molecule SERS events. We present here a specific example of two-analyte SM-SERS based on the isotopic substitution of a methyl ester rhodamine dye. The dyes are carefully characterized (in both standard and SERS conditions) to confirm experimentally their similar chemical properties. We then demonstrate their utility for bi-analyte SERS (BiASERS) experiments and, as an example, highlight the transition from a single, to a few, to many molecules in the statistics of SM-SERS signals. 


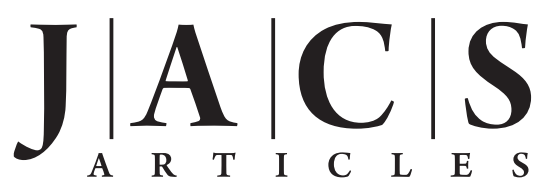

Published on Web 01/23/2009

\section{Evidence of Natural Isotopic Distribution from Single-Molecule SERS}

Pablo G. Etchegoin, ${ }^{*}$ Eric C. Le Ru, ${ }^{*}$ and Matthias Meyer*

The MacDiarmid Institute for Advanced Materials and Nanotechnology, School of Chemical and

Physical Sciences, Victoria University of Wellington, P.O. Box 600, Wellington, New Zealand

Received November 20, 2008; E-mail: Pablo.Etchegoin@vuw.ac.nz; Eric.LeRu@vuw.ac.nz; kiwimatto@gmail.com

Abstract: We report on the observation of the natural isotopic spread of carbon from single-molecule surfaceenhanced Raman spectroscopy (SM-SERS). By choosing a dye molecule with a very localized Ramanactive vibration in a cyano bond $(\mathrm{C} \equiv \mathrm{N}$ triple bond), we observe (in a SERS colloidal liquid) a small fraction of SM-SERS events where the frequency of the cyano mode is softened and in agreement with the effect of substituting ${ }^{12} \mathrm{C}$ by the next most abundant isotope, ${ }^{13} \mathrm{C}$. This example adds another demonstration of single-molecule sensitivity in SERS through isotopic editing, which in this case is done not by artificial isotopic editing but rather by nature itself. It also highlights SERS as a unique spectroscopic tool that is capable of detecting an isotopic change in one atom of a single molecule. 


\section{Bibliography}

[1] C. V. Raman. A new radiation. Indian J. Phys., 2:387-398, 1928.

[2] C. V. Raman and K. S. Krishnan. A new type of secondary radiation. Nature, 121:501-502, 1928.

[3] D. A. Long. The Raman Effect, A Unified Treatment of the Theory of Raman Scattering by Molecules. John Wiley \& Sons Ltd., Chichester, 2002.

[4] M. Fleischmann, P. J. Hendra, and A. J. McQuillan. Raman spectra of pyridine adsorbed at a silver electrode. Chem. Phys. Lett., 26:163, 1974.

[5] David L. Jeanmaire and Richard P. Van Duyne. Surface raman spectroelectrochemistry part i. Heterocyclic, aromatic, and aliphatic amines adsorbed on the anodized silver electrode. J. Electroanal. Chem., 84:1-20, 1977.

[6] M. Grant Albrecht and J. Alan Creighton. Anomalously intense Raman spectra of pyridine at a silver electrode. J. Am. Chem. Soc., 99(15):5215, 1977.

[7] M. R. Philpott. Effect of surface plasmons on transitions in molecules. J. Chem. Phys., 62:1812, 1975.

[8] Katrin Kneipp, Y. Wang, Harald Kneipp, L. T. Perelman, I. Itzkan, R. R. Dasari, and M. S. Feld. Single molecule detection using surface- 
enhanced Raman scattering (SERS). Phys. Rev. Lett., 78:1667-1670, 1997.

[9] Shuming Nie and Steven R. Emory. Probing single molecules and single nanoparticles by surface-enhanced Raman scattering. Science, 275(5303):1102-1106, 1997.

[10] T. Hirschfeld. Optical microscopic observation of single small molecules. Appl. Opt., 15(12):2965-2966, 1976.

[11] Shimon Weiss. Fluorescence spectroscopy of single biomolecules. Science, 283:1676-1683, 1999.

[12] Steven R. Emory, Shuming Nie, Katrin Kneipp, and G. R. Harrison. Single-molecule Raman spectroscopy: fact or fiction? Chimia, 53:35, 1999.

[13] Martin Moskovits, Li-Lin Tay, Jody Yang, and Thomas L. Haslett. SERS and the single molecule. In Optical Properties of Nanostructured Random Media, volume 82 of Topics in Applied Physics, pages 215-227. Springer Berlin / Heidelberg, Berlin, 2002.

[14] Eric C. Le Ru, Matthias Meyer, and Pablo G. Etchegoin. Proof of single-molecule sensitivity in surface enhanced Raman scattering (SERS) by means of a two-analyte technique. J. Phys. Chem. B, 110:1944-1948, 2006.

[15] J. W. Verhoeven. Glossary of terms used in photochemistry (IUPAC recommendations 1996). Pure Appl. Chem., 68(12):2223-2286, 1996.

[16] Ricardo F. Aroca. Surface-Enhanced Vibrational Spectroscopy. John Wiley \& sons, Chichester, 2006.

[17] Eric C. Le Ru and Pablo G. Etchegoin. Principles of Surface-Enhanced Raman Spectroscopy and Related Plasmonic Effects. Elsevier, Amsterdam, 2009. 
[18] W. D. Gwinn. Normal coordinates: General theory, redundant coordinates, and general analysis using electronic computers. J. Chem. Phys., 55(2):477, 1971.

[19] Paul J. G. Goulet, Nicholas P. W. Pieczonka, and Ricardo F. Aroca. Overtones and combinations in single-molecule surface-enhanced resonance Raman scattering spectra. Anal. Chem., 75(8):1918-1923, 2003.

[20] R. F. Kubin and A. N. Fletcher. Fluorescence quantum yields of some rhodamine dyes. Journal of Luminescence, 27(4):455-462, 1983.

[21] Eric C. Le Ru, E. Blackie, Matthias Meyer, and Pablo G. Etchegoin. Surface enhanced Raman scattering enhancement factors: A comprehensive study. J. Phys. Chem. C, 111:13794-13803, 2007.

[22] Duncan Graham, Clare McLaughlin, Gerard McAnally, Joanna C. Jones, Peter C. White, and W. Ewen Smith. Synthesis of novel monoazo benzotriazole dyes specifically for surface enhanced Raman scattering. Chem. Commun., 11:1187, 1998.

[23] P. C. Lee and D. Meisel. Adsorption and surface-enhanced Raman of dyes on silver and gold sols. J. Phys. Chem., 86(17):3391-3395, 1982.

[24] J. Alan Creighton, C. G. Blatchford, and M. Grant Albrecht. Plasma resonance enhancement of Raman scattering by pyridine adsorbed on silver or gold sol particles of size comparable to the excitation wavelength. J. Chem. Soc.: Faraday Trans., 2:790-798, 1979.

[25] Martin Moskovits. Surface-enhanced spectroscopy. Rev. Mod. Phys., 57(3):783-826, 1985.

[26] Katrin Kneipp and Harald Kneipp. SERS signals at the anti stokes side of the excitation laser in extremely high local optical fields of silver and gold nanoclusters. Faraday Disc., 132:27-33, 2006. 
[27] Yoshitaka Sawai, Baku Takimoto, Hideki Nabika, Katsuhiro Ajito, and Kei Murakoshi. Observation of a small number of molecules at a metal nanogap arrayed on a solid surface using surface-enhanced Raman scattering. J. Am. Chem. Soc., 129:1658-1662, 2007.

[28] Tamitake Itoh, Kazuhiro Hashimoto, and Yukihiro Ozaki. Polarization dependences of surface plasmon bands and surface-enhanced Raman bands of single ag nanoparticles. Applied Physics Letters, 83(11):2274-2276, 2003.

[29] Mikael Käll, Hongxing Xu, and Peter Johansson. Field enhancement and molecular response in surface-enhanced Raman scattering and fluorescence spectroscopy. J. Raman Spectrosc., 36:510-514, 2005.

[30] Peter Johansson, Hongxing Xu, and Mikael Käll. Surface-enhanced Raman scattering and fluorescence near metal nanoparticles. Phys. Rev. B, 72(3):035427-1-17, 2005.

[31] Hongxing Xu, E. J. Bjerneld, Mikael Käll, and L. Börjesson. Spectroscopy of single hemoglobin molecules by surface enhanced Raman scattering. Phys. Rev. Lett., 83:4357-4360, 1999.

[32] Eric C. Le Ru and Pablo G. Etchegoin. Rigorous justification of the $|E|^{4}$ enhancement factor in surface enhanced Raman spectroscopy. Chem. Phys. Lett., 423:63-66, 2006.

[33] C. F. Bohren and D. R. Huffman. Absorption and Scattering of Light by Small Particles. Wiley, New York, 1983.

[34] H. Chew. Transition rates of atoms near spherical surfaces. J. Chem. Phys., 87:1355-1360, 1987.

[35] W. B. Cai, Bin Ren, X. Q. Li, C. X. She, F. M. Liu, X. W. Cai, and Zhong-Qun Tian. Investigation of surface-enhanced Raman scattering from platinum electrodes using a confocal Raman microscope: dependence of surface roughening pretreatment. Surf. Sci., 406:9-22, 1998. 
[36] P. B. Johnson and R. W. Christy. Optical constants of the noble metals. Phys. Rev. B, 6(12):4370-4379, 1972.

[37] E. D. Palik, editor. Handbook of Optical Constants of Solids III. Academic Press, New York, 1998.

[38] J. R. Sambles, G. W. Bradbery, and F. Yang. Optical excitation of surface plasmons: an introduction. Contemp. Phys., 32(3):173-183, 1991.

[39] Pablo G. Etchegoin, Eric C. Le Ru, and Matthias Meyer. An analytic model for the optical properties of gold. J. Chem. Phys., 125(16):164705, 2006.

[40] Pablo G. Etchegoin, Eric C. Le Ru, and Matthias Meyer. Erratum: "An analytic model for the optical properties of gold" J. Chem. Phys. 125, 164705 (2006). J. Chem. Phys., 127(18):189901, 2007.

[41] P. Etchegoin, J. Kircher, and M. Cardona. Elasto-optical constants of Si. Phys. Rev. B, 47(16):10292-10303, 1993.

[42] A. Bondeson, Thomas Rylander, and Pär Ingelström. Computational Electromagnetics. Springer, 2005.

[43] E. M. Purcell and C. R. Pennypacker. Scattering and absorption of light by nonspherical dielectric grains. The Astrophysical Journal, 186:705-714, 1973.

[44] Bruce T. Draine and Piotr J. Flatau. Discrete-dipole approximation for scattering calculations. J. Opt. Soc. Am. A, 11(4):1491-1499, 1994.

[45] A. L. Gonzalez, Cecilia Noguez, G. P. Ortiz, and G. RodriguezGattorno. Optical absorbance of colloidal suspensions of silver polyhedral nanoparticles. J. Phys. Chem. B, 109:17512-17517, 2005.

[46] Allen Taflove and Susan C. Hagness. Computational Electrodynamics: The Finite-Difference Time-Domain Method. Artech House Publishers, 3 edition, 2005. 
[47] Karl S. Kunz and Raymond J. Luebbers. Finite Difference Time Domain Method for Electromagnetics. CRC-Press, 1 edition, 1993.

[48] Peter Monk. Finite Element Methods for Maxwell's Equations. Oxford University Press, 2003.

[49] John L. Volakis, Arindam Chatterjee, and Leo C. Kempel. Finite Element Method for Electromagnetics. Wiley-IEEE, 1998.

[50] Exemplary open source implementations are, for DDA, DDSCAT (http://www.astro.princeton. edu/ draine/DDSCAT.html), for FDTD, MEEP (http://ab-initio.mit.edu/meep/) and for FEM, elmer (http://www.csc.fi/english/pages/elmer) and femlisp (http://www. femlisp.org/).

[51] Gustav Mie. Beiträge zur Optik trüber Medien, speziell kolloidaler Metallösungen. Ann. Phys. Leipzig, 25(3):377-445, 1908. English title: Articles on the optical characteristics of turbid tubes, especially colloidal metal solutions.

[52] See sections 9.22-9.27 in Ref. [57].

[53] See section 10.4 in Ref. [58].

[54] R. Ruppin. Optical properties of small metal spheres. Phys. Rev. B, 11(8):2871-2876, 1975.

[55] J. M. Gérardy and M. Ausloos. Absorption spectrum of clusters of spheres from the general solution of Maxwells equations.the longwavelength limit. Phys. Rev. B, 22(6):4950-4959, 1980.

[56] J. M. Gérardy and M. Ausloos. Absorption spectrum of clusters of spheres from the general solution of Maxwell's equations. II. optical properties of aggregated metal spheres. Phys. Rev. B, 25(6):4204-4229, 1982.

[57] A. Stratton. Electromagnetic Theory. McGraw-Hill, New York, 1941. 
[58] J. D. Jackson. Classical Electrodynamics. Wiley, New York, 3 edition, 1998.

[59] M. Born and E. Wolf. Principles of Optics. Cambridge University Press, Cambridge, $7^{\text {th }}$ (expanded) edition, 1999.

[60] John Lekner. Theory of Reflection: of Electromagnetic and Particle Waves, volume 3 of Developments in electromagnetic theory and applications 3. M. Nijhoff Publishers ; Kluwer Academic Publishers, Dordrecht ; Boston : Hingham, MA, USA, 1987.

[61] Pablo G. Etchegoin, Chris M. Galloway, and Eric C. Le Ru. Polarization-dependent effects in surface-enhanced Raman scattering (sers). Phys. Chem. Chem. Phys., 8:2624-2628, 2006.

[62] J. P. Kottmann, O. J. F. Martin, D. R. Smith, and S. Schultz. Field polarization and polarization charge distributions in plasmon resonant nanoparticles. New J. Phys., 2:27.1-27.9, 2000.

[63] H. X. Xu and Mikael Käll. Polarization-dependent surface-enhanced Raman spectroscopy of isolated silver nanoaggregates. Chem. Phys. Chem., 4:1001-1005, 2003.

[64] See section 3.4.3 in Ref. [17].

[65] Hongxing Xu. Surface Plasmon Photonics: From Optical Properties of Nanoparticles to Single Molecule Surface-enhanced Raman Scattering. PhD thesis, Chalmers University of Technology, 2002.

[66] Eric C. Le Ru, Pablo G. Etchegoin, and Matthias Meyer. Enhancement factor distribution around a single surface-enhanced Raman scattering hot spot and its relation to single molecule detection. J. Chem. Phys., 125:204701, 2006.

[67] Hongxing Xu, Xue-Hua Wang, Martin P. Persson, H. Q. Xu, Mikael Käll, and Peter Johansson. Unified treatment of fluorescence and Raman scattering processes near metal surfaces. Phys. Rev. Lett., 93(24):243002, 2004. 
[68] Eric C. Le Ru, Pablo G. Etchegoin, J. Grand, N. Félidj, J. Aubard, and G. Lévi. The mechanisms of spectral profile modification in surface enhanced fluorescence. J. Phys. Chem. C, 111(44):16076-16079, 2007.

[69] Martin Moskovits. Surface selection rules. J. Chem. Phys., 77:4408$4416,1982$.

[70] Vickie M. Hallmark and Alan Campion. A modification of the image dipole selection rules for surface Raman scattering. The Journal of Chemical Physics, 84(6):2942-2944, 1986.

[71] Martin Moskovits, D. P. DiLella, and K. J. Maynard. Surface Raman spectroscopy of a number of cyclic aromatic molecules adsorbed on silver: selection rules and molecular reorientation. Langmuir, 4:6776, 1988.

[72] Eric C. Le Ru, Matthias Meyer, E. Blackie, and Pablo G. Etchegoin. Advanced aspects of electromagnetic SERS enhancement factors at a hot-spot. J. Raman Spectrosc., 39:1127-1134, 2008.

[73] Vilfredo Pareto. Cours d'Economie Politique, volume 2. Universite de Lausanne, Lausanne, 1897.

[74] R. A. Fairthorne. Empirical hyperbolic distributions (bradford-zipfmandelbrot) for bibliometric description and prediction. Journal of Documentation, 25:319-343, 1969.

[75] Pablo G. Etchegoin, Matthias Meyer, and Eric C. Le Ru. Statistics of single molecule SERS signals: is there a Poisson distribution of intensities? Phys. Chem. Chem. Phys., 9:3006-3010, 2007.

[76] J. R. Lombardi, R. L. Birke, T. Lu, and J. Xu. Charge-transfer theory of surface enhanced Raman spectroscopy: Herzberg-Teller contributions. J. Chem. Phys., 84:4174, 1986.

[77] Alan Campion, J. E. Ivanecky, C. M. Child, and M. Foster. On the mechanism of chemical enhancement in surface-enhanced Raman scattering. J. Am. Chem. Soc., 117:11807-11808, 1995. 
[78] Alan Campion and P. Kambhampati. Surface-enhanced Raman scattering. Chem. Soc. Rev., 27:241-250, 1998.

[79] Katrin Kneipp. Surface-enhanced Raman scattering. Physics Today, page 40, November 2007.

[80] Pablo G. Etchegoin, Eric C. Le Ru, and Matthias Meyer. SERS assertions addressed. Physics Today, page 40, August 2008.

[81] Katrin Kneipp. Reply to "SERS assertions addressed". Physics Today, pages 14-15, August 2008.

[82] William of Ockham. Quaestiones et decisiones in quattuor libros Sententiarum Petri Lombardi. ed. Lugd., 1495. "Numquam ponenda est pluralitas sine necessitate" (Plurality is not to be posited without necessity).

[83] Andreas Otto, A. Bruckbauer, and Y. X. Chen. On the chloride activation in SERS and single molecule SERS. J. Mol. Struct., 661-662:501$514,2003$.

[84] Siegfried Schneider, Harald Grau, Peter Halbig, P. Freunscht, and Ulrich Nickel. Stabilization of silver colloids by various types of anions and their effect on the surface-enhanced Raman spectra of organic dyes. J. Raman Spectrosc., 27:57-68, 1996.

[85] J. Th. G. Overbeek. Colloid Science, volume 1, page 289. Elsevier, Amsterdam, 1952.

[86] R. J. Hunter. Foundations of Colloid Science, Vols. I and II. Oxford University Press, Oxford, 1989.

[87] Steven E. J. Bell and Narayana M. S. Sirimuthu. Surface-enhanced Raman spectroscopy as a probe of competitive binding by anions to citrate-reduced silver colloids. J. Phys. Chem. A, 109:7405-7410, 2005. 
[88] Hanna Bengter, Charbel Tengroth, and Sven P. Jacobsson. New light on Ag-colloid preparation for surface-enhanced FT-Raman spectroscopy: the role of aggregation. J. Raman Spectrosc., 36:1015-1022, 2005.

[89] Zhong-Qun Tian. General discussions. Faraday Discuss., 132:147-158, 2006.

[90] Mark J. Stevens, Michael L. Falk, and Mark O. Robbins. Interactions between charged spherical macroions. J. Chem. Phys., 104(13):5209$5219,1996$.

[91] I. DÁmico and H. Löwen. Effective forces between macroions: a Monte-Carlo study. Physica A, 237:25-30, 1997.

[92] D. Fennell Evans and Håkan Wennerström. The Colloidal Domain: Where Physics, Chemistry, Biology, and Technology Meet. Wiley-VHC, New York, 1999.

[93] Jacob N. Israelachvili. Intermolecular and Surface Forces. Academic Press, 2 edition, 1992.

[94] J. Hu, Bing Zhao, W. Xu, Y. Fan, B. Li, and Yukihiro Ozaki. Simple method for preparing controllably aggregated silver particle films used as surface-enhanced Raman scattering active substrates. Langmuir, 18:6839-6844, 2002.

[95] X. L. Li, W. Q. Wu, J. H. Zhang, H. Y. Jia, B. Yang, B. Zhao, B. F. Li, and Yukihiro Ozaki. Self-assembled metal colloid films: Two approaches for preparing new SERS active substrates. Langmuir, 20:1298-1304, 2004.

[96] Yu Lu, G. L. Liu, and L. P. Lee. High-density silver nanoparticle film with temperature-controllable interparticle spacing for a tunable surface enhanced Raman scattering substrate. Nano Lett., 5:5-9, 2005.

[97] Martin Moskovits. Surface-enhanced Raman spectroscopy: a brief retrospective. J. Raman Spectrosc., 36:485-496, 2005. 
[98] E. J. Verwey and J. Th. G. Overbeek. Theory of the Stability of Lyophobic Colloids. Elsevier, Amsterdam, 1948.

[99] F. London. The general theory of molecular forces. Trans. Faraday Soc., 33(2):8b-26, 1937.

[100] B. A. Pailthorpe and W. B. Russel. The retarded van der Waals interaction between spheres. J. Colloid Interf. Sci., 89:563, 1982.

[101] Robert Brown. A brief account of microscopical observations made in the months of june, july and august, 1827, on the particles contained in the pollen of plants; and on the general existence of active molecules in organic and inorganic bodies. Phil. Mag., 4:161-173, 1829.

[102] Edward Nelson. Dynamical Theories of Brownian Motion. Princeton University Press., 2 edition, 2001.

[103] Michael Faraday. The Bakerian lecture: experimental relations of gold (and other metals) to light. Phil. Transact. Royal Soc. London, 147:145-181, 1847.

[104] John C. Crocker and David G. Grier. Interactions and dynamics in charge-stabilized colloid. MRS Bulletin, 23(10):24-31, 1998.

[105] Deszö Boda, W. Ronald Fawcett, Douglas Henderson, and Stefan Sokolowski. Monte Carlo, density functional theory, and PoissonBoltzmann theory study of the structure of an electrolyte near an electrode. J. Chem. Phys., 116(16):7170-7176, 2002.

[106] Andreas Otto. The 'chemical' (electronic) contribution to surfaceenhanced Raman scattering. J. Raman Spectrosc., 36:497-509, 2005.

[107] Karen Faulds, Rachael E. Littleford, Duncan Graham, Geoffrey Dent, and W. Ewen Smith. Comparison of surface-enhanced resonance Raman scattering from unaggregated and aggregated nanoparticles. Anal. Chem., 76:592-598, 2004. 
[108] Eric C. Le Ru, Chris M. Galloway, and Pablo G. Etchegoin. On the connection between optical absorption/extinction and SERS enhancements. Phys. Chem. Chem. Phys., 8:3083-3087, 2006.

[109] P. Hildebrandt and M. Stockburger. Surface-enhanced resonance Raman spectroscopy of rhodamine $6 \mathrm{~g}$ adsorbed on colloidal silver. J. Phys. Chem., 88:5935, 1984.

[110] Z. Zhou, G. Wang, and Z. Xu. Single-molecule detection in a liquid by surface-enhanced resonance Raman scattering. Appl. Phys. Lett., 88:034104-034106, 2006.

[111] Catalin C. Neacsu, Jens Dreyer, Nicolas Behr, and Markus B. Raschke. Scanning-probe Raman spectroscopy with single-molecule sensitivity. Phys. Rev. B, 73:193406, 2006.

[112] Katrin Kneipp and Harald Kneipp. Single molecule Raman scattering. Appl. Spectrosc., 60(12):322A-334A, 2006.

[113] Pablo G. Etchegoin, Matthias Meyer, E. Blackie, and Eric C. Le Ru. Statistics of single-molecule surface enhanced Raman scattering signals: Fluctuation analysis with multiple analyte techniques. Anal. Chem., 79:8411-8415, 2007.

[114] Amy M. Michaels, Jiang Jiang, and Louis E. Brus. Ag nanocrystal junctions as the site for surface-enhanced Raman scattering of single rhodamine 6g molecules. J. Phys. Chem. B, 104:11965-11971, 2000.

[115] A. Weiss and Gilad Haran. Time-dependent single-molecule Raman scattering as a probe of surface dynamics. J. Phys. Chem. B, 105:1234812354, 2001.

[116] Pablo G. Etchegoin, Rob C. Maher, L. F. Cohen, H. J. N. Hartigan, R. J. C. Brown, M. J. T. Milton, and J. C. Gallop. New limits in ultrasensitive trace detection by surface enhanced Raman scattering (SERS). Chem. Phys. Lett., 375:84-90, 2003. 
[117] Yamit Sharaabi, Timur O. Shegai, and Gilad Haran. Two-state analysis of single-molecule Raman spectra of crystal violet. Chem. Phys., 318:44-49, 2005.

[118] Timur O. Shegai and Gilad Haran. Probing the Raman scattering tensors of individual molecules. J. Phys. Chem. B, 110(6):2459-2461, 2006.

[119] Andreas Otto. What is observed in single molecule SERS, and why? J. Raman Spectrosc., 33:593-598, 2002.

[120] S. Corni and J. Tomasi. Surface enhanced Raman scattering from a single molecule adsorbed on a metal particle aggregate: A theoretical study. J. Chem. Phys., 116(3):1156-1164, 2002.

[121] William E. Doering and Shuming Nie. Single-molecule and single nanoparticle SERS: Examining the roles of surface active sites and chemical enhancement. J. Phys. Chem. B, 106:311-317, 2002.

[122] Rob C. Maher, Mike Dalley, Eric C. Le Ru, L. F. Cohen, Pablo G. Etchegoin, H. J. N. Hartigan, R. J. C. Brown, and M. J. T. Milton. Physics of single molecule fluctuations in surface enhanced Raman spectroscopy active liquids. J. Chem. Phys., 121(18):8901-8910, 2004.

[123] Andreas Otto. On the significance of Shalaevś hot spots in ensemble and single-molecule SERS by adsorbates on metallic films at the percolation threshold. J. Raman Spectrosc., 37(9):937-947, 2006.

[124] Masayuki Futamata. Single molecule sensitivity in SERS: importance of junction of adjacent Ag nanoparticles. Faraday Disc., 132:45-61, 2006.

[125] C. J. L. Constantino, T. Lemma, P. A. Antunes, and Ricardo F. Aroca. Single-molecule detection using surface-enhanced resonance Raman scattering and Langmuir-Blodgett monolayers. Anal. Chem., 73:36743678, 2001. 
[126] Paul J. G. Goulet, Nicholas P. W. Pieczonka, and Ricardo F. Aroca. Mapping single-molecule SERRS from Langmuir-Blodgett monolayers on nanostructured silver island films. J. Raman Spectrosc., 36:574580, 2005.

[127] Paul J. G. Goulet and Ricardo F. Aroca. Distinguishing individual vibrational fingerprints: Single-molecule surface-enhanced resonance Raman scattering from one-to-one binary mixtures in langmuirblodgett monolayers. Anal. Chem., 79:2729-2734, 2007.

[128] B. Sick, B. Hecht, U. P. Wild, and L. Novotny. Probing confined fields with single molecules and vice versa. J. Microsc., 202:365-373, 2001.

[129] Bin Ren, Gennaro Picardi, Bruno Pettinger, Rolf Schuster, and Gerhard Ertl. Tip-enhanced Raman spectroscopy of benzenethiol adsorbed on $\mathrm{Au}$ and Pt single-crystal surfaces. Angew. Chem. Int. Ed., 44:139-142, 2005.

[130] Bruno Pettinger, Gennaro Picardi, Rolf Schuster, and Gerhard Ertl. Surface-enhanced and STM-tip-enhanced Raman spectroscopy at metal surfaces. Single Mol., 5-6:285-294, 2002.

[131] Bruno Pettinger, Bin Ren, Gennaro Picardi, Rolf Schuster, and Gerhard Ertl. Tip-enhanced Raman spectroscopy (ters) of malachite green isothiocyanate at $\mathrm{Au}(111)$ : bleaching behavior under the influence of high electromagnetic fields. J. Raman Spectrosc., 36:541-550, 2005.

[132] Jens Steidtner and Bruno Pettinger. Tip-enhanced Raman spectroscopy and microscopy on single dye molecules with $15 \mathrm{~nm}$ resolution. Physical Review Letters, 100(23):236101, 2008.

[133] Katrin F. Domke and Bruno Pettinger. Comment on "Scanning-probe Raman spectroscopy with single-molecule sensitivity". Phys. Rev. B, 75:236401-236403, 2007. 
[134] Catalin C. Neacsu, Jens Dreyer, Nicolas Behr, and Markus B. Raschke. Reply to "Comment on 'Scanning-probe Raman spectroscopy with single-molecule sensitivity"'. Phys. Rev. B, 75:236402-236405, 2007.

[135] Rob C. Maher, L. F. Cohen, and Pablo G. Etchegoin. Single molecule photo-bleaching observed by surface enhanced resonant Raman scattering (SERRS). Chem. Phys. Lett., 352:378-384, 2002.

[136] D. Zhang, Y. Xie, Shirshendu K. Deb, V. Jo Davisson, and D. BenAmotz. Isotope edited internal standard method for quantitative surface-enhanced Raman spectroscopy. Anal. Chem., 77(11):35633569, 2005.

[137] F. Svedberg and Mikael Käll. On the importance of optical forces in surface-enhanced Raman scattering (SERS). Faraday Discuss., 132:3544, 2006.

[138] F. Svedberg, Z. Li, Hongxing Xu, and Mikael Käll. Creating hot nanoparticle pairs for surface-enhanced Raman spectroscopy through optical manipulation. Nano Lett., 6(12):2639-2641, 2006.

[139] B. Sepúlveda, J. Alegret, and Mikael Käll. Nanometric control of the distance between plasmonic nanoparticles using optical forces. Optics Express, 15(22):14914-14920, 2007.

[140] Pablo G. Etchegoin, Paul D. Lacharmoise, and Eric C. Le Ru. Influence of photostability on single-molecule surface enhanced Raman scattering enhancement factors. Anal. Chem., 81:682-688, 2009.

[141] T. A. Witten and L. M. Sander. Diffusion-limited aggregation, a kinetic critical phenomenon. Phys. Rev. Lett., 47(19):1400-1403, 1981.

[142] Jorg Zimmermann, Ullrich Siggel, Jurgen-Hinrich Fuhrhop, and Beate Roder. Excitonic coupling between $B$ and $Q$ transitions in a porphyrin aggregate. The Journal of Physical Chemistry B, 107(25):60196021, 2003. 
[143] J. R. Lakowicz. Principles of Fluorescence Spectroscopy. Springer, New York, $3^{\text {rd }}$ ed. edition, 2006.

[144] Eric C. Le Ru, Mike Dalley, and Pablo G. Etchegoin. Plasmon resonances of silver colloids studied by surface enhanced Raman spectroscopy. Current Appl. Phys., 6:411, 2006.

[145] Jon A. Dieringer, Robert B. Lettan II, Karl A. Scheidt, and Richard P. Van Duyne. A frequency domain existence proof of single-molecule surface-enhanced Raman spectroscopy. J. Am. Chem. Soc., 129:1624916256, 2007.

[146] Shirshendu K. Deb, Brandon Davis, D. Ben-Amotz, and V. Jo Davisson. Accurate concentration measurements using surfaceenhanced Raman and deuterium exchanged dye pairs. Appl. Spectrosc., 62(9):1001-1007, 2008.

[147] Richard van Duyne's plenary talk at the ICORS 2006 conference in Japan, Yokohama.

[148] E. Blackie, Eric C. Le Ru, Matthias Meyer, Mattie Timmer, Brandon Burkett, Peter Northcote, and Pablo G. Etchegoin. Bi-analyte SERS with isotopically edited dyes. Phys. Chem. Chem. Phys., 10:4147-4153, 2008.

[149] Pablo G. Etchegoin, Eric C. Le Ru, and Matthias Meyer. Evidence of natural isotopic distribution from single-molecule SERS. Journal of the American Chemical Society, 131(7):2713-2716, 2009.

[150] J. R. de Laeter, J. K. Böhlke, P. De Bièvre, H. Hidaka, H. S. Peiser, K. J. R. Rosman, and P. D. P. Taylor. Atomic weights of the elements. Review 2000 (IUPAC technical report). Pure Appl. Chem., 75(6):683799, 2003.

[151] Warren J. Hehre, R. F. Stewart, and John A. Pople. Self-consistent molecular-orbital methods. i. use of Gaussian expansions of Slatertype atomic orbitals. J. Chem. Phys., 51(6):2657-2664, 1969. 
[152] R. Krishnan, J. Stephen Binkley, R. Seeger, and John A. Pople. Selfconsistent molecular orbital methods. XX. a basis set for correlated wave functions. J. Chem. Phys., 72:650-654, 1980.

[153] J. Stephen Binkley, John A. Pople, and Warren J. Hehre. Selfconsistent molecular orbital methods. 21. small split-valence basis sets for first-row elements. J. Am. Chem. Soc., 102(3):939-947, 1980.

[154] Mark S. Gordon, J. Stephen Binkley, John A. Pople, William J. Pietro, and Warren J. Hehre. Self-consistent molecular-orbital methods. 22. small split-valence basis sets for second-row elements. J. Am. Chem. Soc., 104(10):2797-2803, 1982.

[155] C. Lee, W. Yang, and R. G. Parr. Development of the Colle-Salvetti correlation-energy formula into a functional of the electron density. Phys. Rev. B, 37:785-789, 1988.

[156] Gordon E. Moore. Cramming more components onto integrated circuits. Electronics, 38(8), 1965. Moore's law.

[157] M. W. Schmidt, K. K. Baldridge, J. A. Boatz, S. T. Elbert, Mark S. Gordon, J. H. Jensen, S. Koseki, N. Matsunaga, K. A. Nguyen, S. Su, T. L. Windus, Michel Dupuis, and J. A. Montgomery. General atomic and molecular electronic structure system. J. Comput. Chem., 14:13471363, 1993.

[158] M. J. Frisch, G. W. Trucks, H. B. Schlegel, G. E. Scuseria, M. A. Robb, J. R. Cheeseman, J. A. Montgomery, K. N. Kudin, T. Vreven, J. C. Burant, J. M. Millam, S. S. Iyengar, J. Tomasi, V. Barone, B. Mennucci, M. Cossi, G. Scalmani, G. A. Petersson, N. Rega, H. Nakatsuji, M. Hada, M. Ehara, R. Fukuda, K. Toyota, J. Hasegawa, M. Ishida, T. Nakajima, Y. Honda, O. Kitao, H. Nakai, M. Klene, X. Li, J. E. Knox, H. P. Hratchian, J. B. Cross, V. Bakken, C. Adamo, J. Jaramillo, R. Gomperts, R. E. Stratmann, O. Yazyev, A. J. Austin, R. Cammi, C. Pomelli, P. Y. Ayala, Joseph W. Ochterski, K. Morokuma, G. A. Voth, P. Salvador, J. J. Dannenberg, V. G. Zakrzewski, S. Dapprich, 
A. D. Daniels, O. Farkas, M. C. Strain, D. K. Malick, A. D. Rabuck, K. Raghavachari, J. B. Foresman, J. V. Ortiz, Q. Cui, A. G. Baboul, S. Clifford, J. Cioslowski, B. B. Stefanov, G. Liu, A. Liashenko, I. Komaromi, P. Piskorz, R. L. Martin, D. J. Fox, T. Keith, M. A. Al-Laham, C. Y. Peng, A. Nanayakkara, M. Challacombe, P. M. W. Gill, B. Johnson, W. Chen, M. W. Wong, C. Gonzalez, and John A. Pople. Gaussian 03, Revision C.02. Gaussian, Inc., Wallingford, CT, 2004.

[159] Thomas Engel. Quantum Chemistry and Spectrocopy. Pearson, 2005.

[160] J. Neugebauer, M. Reiher, C. Kind, and B. A. Heiss. Quantum chemical calculation of vibrational spectra of large molecules - Raman and IR spectra for buckminsterfullerene. J. Computational Chem., 23:895910, 2002.

[161] A. D. Becke. Density-functional thermochemistry. III. the role of exact exchange. J. Chem. Phys., 98:5648-5652, 1993.

[162] H. W. Schroetter and H. W. Kloeckner. Raman scattering cross sections in gases and liquids. In Raman Spectroscopy of Gases and Liquids, volume 11 of Topics in Current Physics, page 123. A. Weber, ed., Spring-Verlag, 1979.

[163] Tamitake Itoh, Kenichi Yoshida, Vasudevanpillai Biju, Yasuo Kikkawa, Mitsuru Ishikawa, and Yukihiro Ozaki. Second enhancement in surface-enhanced resonance Raman scattering revealed by an analysis of anti-Stokes and Stokes Raman spectra. Physical Review B (Condensed Matter and Materials Physics), 76(8):085405, 2007.

[164] Ken-ichi Yoshida, Tamitake Itoh, Vasudevanpillai Biju, Mitsuru Ishikawa, and Yukihiro Ozaki. Experimental evaluation of the twofold electromagnetic enhancement theory of surface-enhanced resonance Raman scattering. Physical Review B (Condensed Matter and Materials Physics), 79(8):085419, 2009. 
[165] P. M. Champion and A. C. Albrecht. Resonance Raman scattering: The multimode problem and transform methods. Ann. Rev. Phys. Chem, 33:353-376, 1982.

[166] Bruce B. Johnson and Warner L. Peticolas. The resonant Raman effect. Ann. Rev. Phys. Chem., 27:465-491, 1976.

[167] Arieh Warshel. Interpretation of the resonance Raman spectra of biological molecules. Ann. Rev. Biophys. Bioeng., 6:273-300, 1977.

[168] Willem Siebrand and Marek Z. Zgierski. The Dushinsky effect in resonance Raman spectroscopy. Chem. Phys. Lett., 62(1):3-8, 1979.

[169] Hiroyuki Watanabe, Norihiko Hayazawa, Yasushi Inouye, and Satoshi Kawata. DFT vibrational calculations of rhodamine $6 \mathrm{~g}$ adsorbed on silver: Analysis of tip-enhanced Raman spectroscopy. J. Phys. Chem. B, 109:5012-5020, 2005.

[170] "Blender", an open source 3D content creation suite: www.blender.org.

[171] "GIMP", the GNU Image Manipulation Program: www.gimp.org.

[172] Pablo G. Etchegoin, M. Cardona, R. Lauck, R. J. H. Clark, J. Serrano, and A. H. Romero. Temperature-dependent Raman scattering of natural and isotopically substituted pbs. Phys. Status Solidi (B), 245(6):1125-1132, 2008.

[173] Pablo G. Etchegoin and Eric C. Le Ru. A perspective on single molecule SERS: current status and future challenges. Phys. Chem. Chem. Phys., 10:6079-6089, 2008. 